Florida International University

FIU Digital Commons

FIU Electronic Theses and Dissertations

University Graduate School

$12-8-2017$

\title{
Top-down and Bottom-up Approaches to Understanding the Fate of the Federally Endangered Schaus' Swallowtail Butterfly (Heraclides aristodemus ponceanus)
}

Jaeson T. Clayborn

Florida International University, jclay010@fiu.edu

DOI: $10.25148 /$ etd.FIDC006567

Follow this and additional works at: https:// digitalcommons.fiu.edu/etd

Part of the Biology Commons, Botany Commons, Entomology Commons, and the Outdoor Education Commons

\section{Recommended Citation}

Clayborn, Jaeson T., "Top-down and Bottom-up Approaches to Understanding the Fate of the Federally Endangered Schaus' Swallowtail Butterfly (Heraclides aristodemus ponceanus)" (2017). FIU Electronic Theses and Dissertations. 3698.

https://digitalcommons.fiu.edu/etd/3698 


\title{
FLORIDA INTERNATIONAL UNIVERSITY
}

Miami, Florida

TOP-DOWN AND BOTTOM-UP APPROACHES TO UNDERSTANDING THE FATE OF THE FEDERALLY ENDANGERED SCHAUS' SWALLOWTAIL BUTTERFLY (HERACLIDES ARISTODEMUS PONCEANUS)

\author{
A dissertation submitted in partial fulfillment of the \\ requirement for the degree of \\ DOCTOR OF PHILOSOPHY \\ in \\ BIOLOGY \\ by \\ Jaeson Tyrone Clayborn \\ 2017
}


To: Dean Michael R. Heithaus

College of Arts, Sciences and Education

This dissertation, written by Jaeson Tyrone Clayborn, and titled Top-down and Bottomup Approaches to Understanding the Fate of the Federally Endangered Schaus' Swallowtail Butterfly (Heraclides aristodemus ponceanus), having been approved in respect to style and intellectual concept, is referred to you for judgement.

We have read this dissertation and recommend that it be approved.

Bradley Bennett

Maureen Donnelly

George O’Brien

Jeffrey Wells

Suzanne Koptur, Major Professor

Date of Defense: December 8, 2017

The dissertation of Jaeson Tyrone Clayborn is approved.

Dean Michael R. Heithaus

College of Arts, Sciences and Education

Andrés G. Gil

Vice President for Research and Econoic Development and Dean of the University Graduate School

Florida International University, 2018 


\section{DEDICATION}

I dedicate this dissertation to my family, friends, scientists, and educators.

Nowadays, the stories we tell about animals and plants matter because those stories might determine the fate of organisms on the brink of extinction.

"In the end, we will conserve only what we love, we will love only what we understand, and we will understand only what we are taught." - Baba Dioum 


\section{ACKNOWLEDGMENTS}

This research was supported in part by DonorsChoose.org, Dissertation Year Fellowship, FIU Global Civic Engagement Grant, Kelly Foundation for Tropical Botany, North American Butterfly Association Miami Blue Research Grant, Tinker Field Research Grant, and the Zoo Miami Research and Conservation Fund.

I thank my wife Yadira, mother-in-law Elia, and father-in-law Ediliso for their love and support through the trials and tribulations of academic research. My wife, in particular, provided comfort and guidance to keep me motivated when, at times, the stress was overwhelming. We were able to brainstorm ideas to improve the research and troubleshoot impediments.

My mother and sister Jasmyn were instrumental in providing the foundation to study animals and plants and pursue higher education. My mother is a nurturer and provides solace when family members are stressed or in despair; however, she was austere when the topic transitioned to education and grades. My curiosity and engagement in nature were never belittled by them, but encouraged. My mother promoted exploration in the sciences through family trips to nature centers, the Cleveland Metroparks, and the Cleveland Metroparks Zoo.

My grandmother, aunt Dorothy, aunt Cassandra, aunt Pam, cousin Robert, cousin Ashli, cousin Chandra, cousin Austin, cousin Romelle Jr., cousin Phillip, cousin-in-law Sheila, and aunt Cynthia were also supportive in my pursuit of scientific exploration and knowledge. They did not deflate my enthusiasm, but allowed me to catch animals in their yards, helped construct aquariums and terrariums, and participated in family activities at the park and zoo. 
Unfortunately, my father passed away before I attended graduate school at the University of South Florida St. Petersburg and Florida International University. He was a veteran electrician in Atlanta, Georgia and avid sports player and fan. He also understood the value of higher education (and education in general) to ascend the economic ladder. His electrical expertise was passed on to my brother Tyson. Tyson assembled the batteries and solar panels network to power high-definition video surveillance cameras in the dry forest at Elliott Key. I grateful for his support to help me explore novel research methods in the field. My youngest brother, Taylor, is a musician and music instructor; however, he also demonstrated a strong affinity towards wildlife. Taylor endured the brutal heat and mosquitoes in Biscayne National Park and volunteered his vacation time to help remove invasive plants at Adams Key. Finally, I thank my stepmother for allowing my brothers and I to harbor plants and animals in the house. She enjoys gardening and showcasing native and exotic plant species in the yard.

My major advisor, Dr. Suzanne Koptur, is the reason I have come this far. She provided the lab space, guidance, mentorship, and optimism for research excellence. I am fortunate to have had the opportunity to conduct research in her lab; and for that, I am grateful. Working in her lab has given me the opportunity and skillset to continue the pursuit of knowledge, advocacy for conservation and preservation, and empathy. John Koptur-Palenchar provided assistance in obtaining obscure documents for the Schaus' swallowtail butterfly at the University of Florida.

I would like to thank my dissertation committee: Dr. Bradley Bennett, Dr. Donnelly, and Dr. O'Brien, and Dr. Wells. They have pushed me to be a better scientist and person. Dr. Donnelly encourages her students to read more (a lot more) and be scientifically up- 
to-date, but also know science history. Dr. Bennett is a scrupulous botanist and challenges his students to be the same in their investigations. Dr. Wells challenged my assertions in the dissertation. He guides his students to state the facts based on sound scientific investigations and statistical analyses, but avoid extrapolated hyperbole. Dr. O'Brien values science education in the classroom and school surroundings. He teaches his pre-services students (teachers) and demonstrates through various activities how to engage students through active-learning, project-based activities, and learning through play and peer-peer discussion. Collectively, they have challenged me to learn explicitly, improve my writing and statistical skills, and enhance my dissertation.

Dr. Kevin Whelan was the principal investigator for the Schaus' Swallowtail Habitat Enhancement Project in Biscayne National Park. Though he was not on my dissertation committee, he was crucial mentor for my dissertation. My research questions aligned with his work and experience at the National Park Service South Florida Caribbean Network. This project was the genesis of my true dissertation. I am truly grateful for his continued support.

Craig Perry, Michael Foguer, and Lydia Cuni were essential staff for the Schaus' Swallowtail Habitat Enhancement Project. They put in long hours to maintain the restoration sites and collect data. The arduous tasks on the islands require commitment and endurance. They also supported my research tangential to the Schaus' Swallowtail Habitat Enhancement Project. Without them, collecting data in the field would have been more challenging than the already challenging work. I would like to thank Vicente Martinez, Shane Harrington, and Steve Newman for their support and transportation to 
the islands. The support and cooperation of Biscayne National Park and the National Park Service staff were essential in project development to completion.

Leslie Bayas, Eduardo Ibarra, Brenda Rivera, and Adriana Samani were undergraduate students at Florida International University. They have since graduated. They also helped me set up experiments, collect data, and persevered through the heat and mosquitoes in the field. Thomas Spencer collected data for an ant-caterpillar lab experiment and committed many hours watching native and exotic ants battle or ignore caterpillars. Darlene Darrican, a high schooler, was a dedicated volunteer in the lab and field. She was effective and a quick-learner. She was also essential in conducting lab and field experiments.

My labmates (old and new): Roxaneh Khorsand Rosa, Beyte Barrios, Ian Jones, Andrea Salas, Cleopatra Pimienta, Brittany Harris, Adel Peña. Collectively, we were supportive of each other's research and brainstormed different ideas or engaged in intellectual discussion. Individually, they provided advice, guidance, and sometimes assisted in fieldwork.

Tanjim Hossain provided ancillary help throughout my entire dissertation. His knowledge and ambitious ideas helped refine some investigations in the dissertation. He also spent ample time in the field and provided volunteers from the University of Miami to help in the Schaus' Swallowtail Habitat Enhancement Project. At the University of Miami, Ryan Champiny was a committed research volunteer. She helped me conduct ant surveys in the field and identify them in the lab. Christine Pardo and Dr. Julia Wester were essential critics for Chapter 4 . Their commitment to survey instruments gave me more insight to improve my surveys and interview questions. 
Dr. Marc Minno is an experienced lepidopterist and surveys butterflies, moths, and skippers throughout the United States and Caribbean. He has been supportive of my research, brainstorming and providing guidance over the years. His writings, survey data, and resources have been invaluable in the pursuit of butterfly preservation and habitat conservation.

Dr. Paulo Oliveira provided invaluable information and guidance on the antcaterpillar interaction study. His legacy (literature, methods, expertise) regarding antcaterpillar-plant investigations in lab and field settings has been indispensable. Dr. Mark Deyrup, at the Archbold Biological Station, demonstrated ant collection techniques and methods to identify ant specimens. He also identified difficult ant specimens collected in the field.

Dr. Scott Zona provided greenhouse space to raise and maintain host and nectar plants for experiments and future butterfly gardens at schools. He is a knowledgeable botanist and experienced writer. Dr. Zona and Dr. Koptur helped improve my writing skills and meticulous interpretation of scientific articles.

The North American Butterfly Association (NABA), Miami Blue Chapter was my lifeline for butterfly investigations. I thank Linda Evans (President), Patty Phares, Elane and Ron Nuehring, Susan Koltermann, and NABA Miami Blue Chapter members. Elane Nuehring passed away during my infancy period as a PhD student (while I was still learning the ropes); consequently, she will not be forgotten, but remembered for her tenacious attitude towards lepidopteran education, preservation, research, and policy. Her mentorship improved my learning curve for south Florida ecosystems. Linda Evans, Patty Phares, Ron Nuehring, Susan Koltermann and many other NABA Miami Blue Chapter 
members taught me how to identify butterflies and plants in south Florida, provided invaluable advice on my investigations, connected me to other butterfly enthusiasts and scientists from other states and countries, and engaged in dialogue about the issues and joys of butterflies, moths, and skippers.

The butterfly gardens constructed at each school would not have been possible without support from Mark Glasser. Mark enjoys butterflies and plants. He, tenaciously, has transformed his community into a butterfly sanctuary by providing plants and information on plant care and butterfly diversity to his neighbors.

Betty Stultz is a retired elementary school teacher. She has been a mentor and brainstormed methods to work with teachers and students in Miami-Dade County. I have also learned crucial information about education and teaching, which has improved my communication skills with the general public.

I thank Denise Alfaro (Sylvania Heights Elementary), Susana Bello (Coral Terrace Elementary), Sarah Davio (Whispering Pines Elementary), Nadia Earl (North Hialeah Elementary), Janine Fernandez, Lourdes Guaty (Gateway Environmental K-8 Learning Center), Jessica Hernandez (North Hialeah Elementary), Sandra Martinez (Air Base K-8 Center), Carmen Monge (Air Base K-8 Center), Kamelia Ramnarine (Whispering Pines Elementary), Vicki Terranova (Whispering Pines Elementary), Louis Vazquez (North Hialeah Elementary). They were involved directly and indirectly in my research. Their support and guidance initiated Chapter 1 which transitioned to Chapter 4 in my dissertation. They volunteered their time and class time and committed to the butterfly garden investigations. I am grateful for the principals at each school: Tamela Brown (Whispering Pines Elementary), Yanelys Canales (North Hialeah Elementary), and Raul 
Calzadilla, Jr. (Air Base K-8 Center). They gave me permission to work on their school grounds and conduct the investigations. Their dedication to environmental science excellence and project-based learning promotes environmental stewardship and academic achievement.

Research in the Caribbean would not have been possible without assistance from Lemuel Familia Rodriguez (Dominican Republic), Rosa Rodriguez (Dominican Republic), Bryan Naqqi Manco (Turks and Caicos), Dr. Jorge Ferrer (Cuba), Maritza Gutierrez (Cuba), Yaritza Gutierrez (Cuba). While there, I was able to learn different techniques to survey butterflies and ants in tropical dry forests. Through field work, I learned about the similarities and differences between the Florida Keys and islands in the Lucayan Archipelago and Caribbean.

Dr. Deby Cassill was my mentor at the University of South Florida St. Petersburg. She continues to support my research and provides expert advice on ant experiments and methods.

I would like to thank Jennifer Morales, Stephanie Garcia, Stefanie Moore, and Jay Sah for method development and statistical consultation. Jennifer Morales helped develop surveys instruments for Chapter 4 .

Finally, I thank Daniel Gann and Mario Londoño for spatial analysis guidance and support. A geographic information system is a useful tool for spatial analysis and visualization in research. Their expertise manipulating the program allowed me to conduct more investigations and speculate the future of dry forest ecosystems in the northern Florida Keys, critical habitat of the federally endangered Schaus' swallowtail butterfly. 


\title{
ABSTRACT OF THE DISSERTATION
}

TOP-DOWN AND BOTTOM-UP APPROACHES TO UNDERSTANDING THE FATE

OF THE FEDERALLY ENDANGERED SCHAUS' SWALLOWTAIL BUTTERFLY

\section{(HERACLIDES ARISTODEMUS PONCEANUS)}

\author{
by \\ Jaeson Tyrone Clayborn \\ Florida International University, 2018 \\ Miami, Florida \\ Professor Suzanne Koptur, Major Professor
}

The federally endangered Schaus' swallowtail butterfly (Heraclides aristodemus ponceanus) populations have declined precipitously over the years. Despite tremendous efforts to augment the numbers of this butterfly through captive-bred releases and habitat enhancements, it remains imperiled and federally endangered. The dissertation's objectives were: 1) to restore dry forest habitat in Biscayne National Park (BNP) for Schaus' swallowtails; 2) to assess host plant (torchwood and wild lime) survivorship and growth in the restoration sites (BNP) and project dry forest habitat loss because of imminent sea level rise (SLR); 3) to quantify ant activity and record predator-prey interactions against Heraclides caterpillars in dry forest habitat in BNP; and 4) to implement a south Florida butterfly conservation curriculum unit at schools in MiamiDade County, and evaluate students' gained knowledge and attitudes toward south Florida environments, insects and butterflies, and conservation.

More than 3,200 native plants were planted in restoration sites in BNP. Eighty-five percent were torchwood, the primary host plant for Schaus' swallowtails. Canopy cover, 
ground elevation, and relative soil depth had a negligible effect on torchwood survivorship; however, ground elevation had a strong effect on growth. Dry forest habitat in Key Largo will succumb less quickly to SLR than in BNP because of higher elevation. Pseudomyrmex gracilis was the most common ant on Schaus' swallowtail host plants, which aggressively attacked early instar caterpillars (86.7\%). The predator exclusion experiment demonstrated that early instar caterpillars were least likely to survive when exposed to crawling and non-crawling predators.

The controlled-experiment at three schools revealed the integrated insect curriculum unit significantly increased students' content knowledge in the experimental groups. Preand post-surveys revealed students demonstrated favorable interest in animals and plants and were advocates for environmental stewardship; however, they reported less interest in insects. Service-learning and inquiry-based activities in dry forests, gardens, homes, and schools make imperiled insects and ecosystems salient, thus leading to potential advocacy for them. A better understanding of Schaus' swallowtail dynamics in the Florida Keys presents an opportunity to preserve its existence and thwart extinction. 


\section{TABLE OF CONTENTS}

CHAPTER

PAGE

INTRODUCTION 1

LITERATURE CITED .5

I. THE SCHAUS' SWALLOWTAIL HABITAT ENHANCEMENT

PROJECT: AN APPLIED SERVICE-LEARNING PROJECT

CONTINUUM FROM BISCAYNE NATIONAL PARK TO MIAMI-

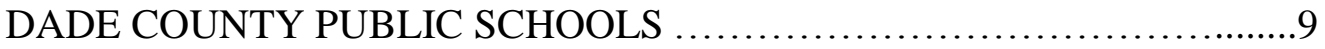

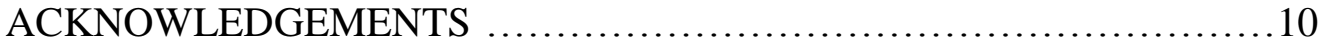

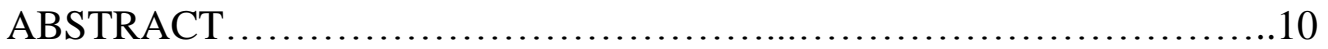

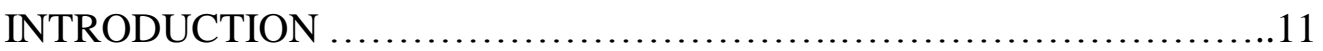

FIELD-SITE DESCRIPTION …......................................

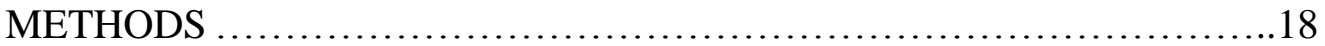

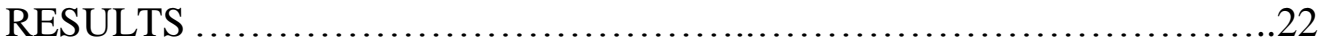

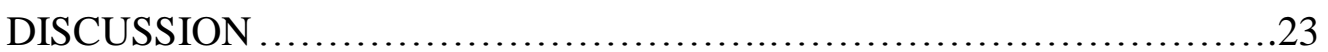

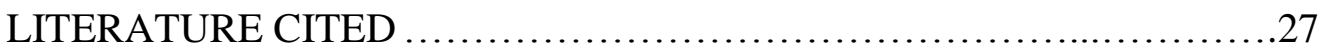

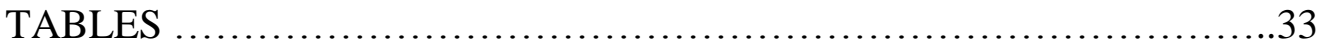

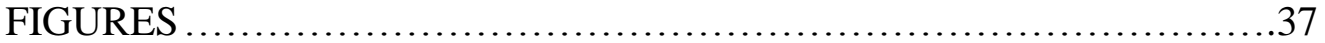

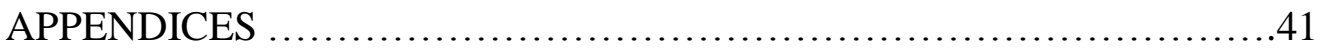

II. RISING SEAS IN THE FLORIDA KEYS: THE LAST REFUGE FOR THE FEDERALLY ENDANGERED SCHAUS' SWALLOWTAIL BUTTERFLY (HERACLIDES ARISTODEMUS PONCEANUS) ..............43

ACKNOWLEDGEMENTS .............................................44

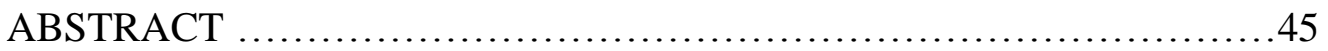

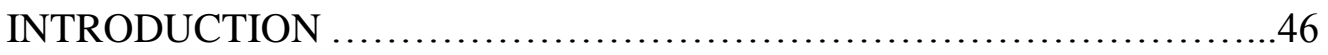

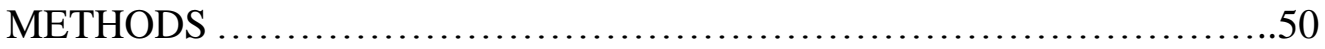

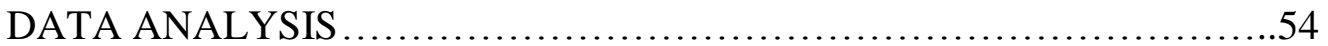

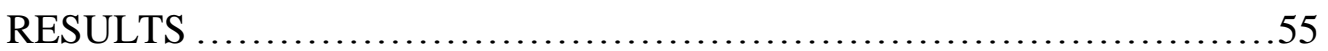

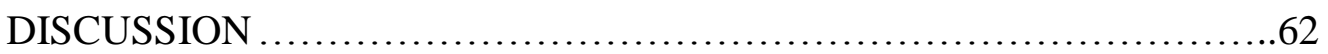

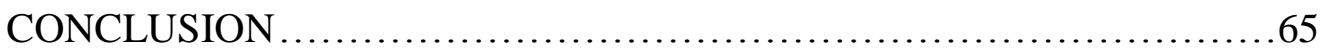

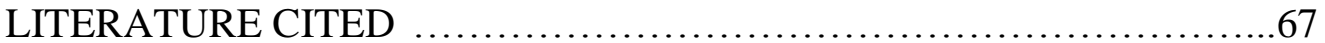

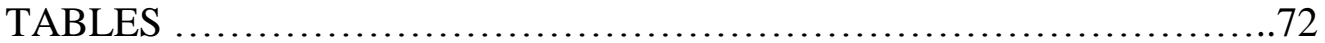

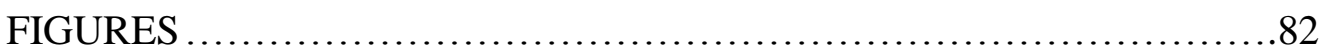

III. MORTAL COMBAT BETWEEN ANTS AND CATERPILLARS: AN

OMINOUS THREAT TO THE ENDANGERED SCHAUS'

SWALLOWTAIL BUTTERFLY (HERACLIDES ARISTODEMUS

PONCEANUS) IN THE FLORIDA KEYS, USA ….....................100

ACKNOWLEDGEMENTS .......................................... 101

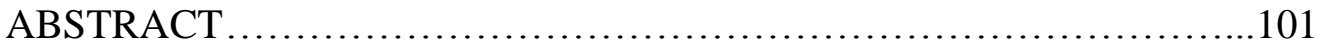

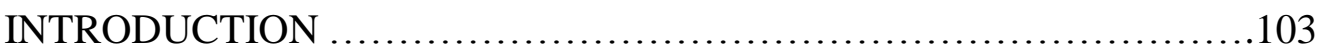




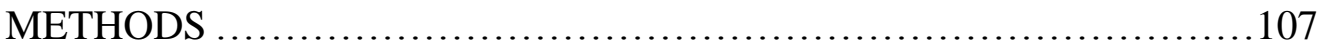

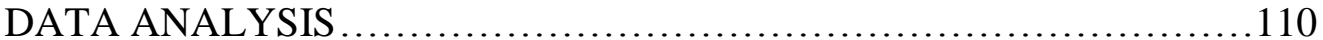

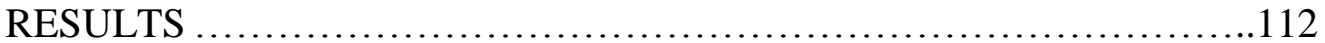

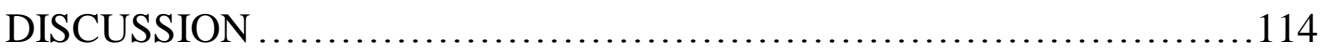

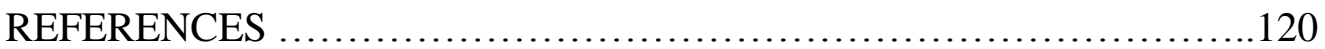

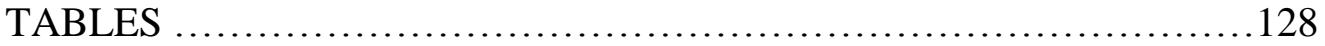

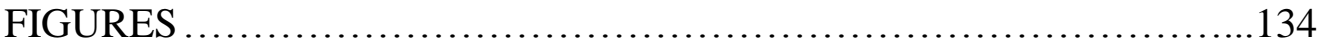

IV. BUTTERFLY GARDENING AT MIAMI URBAN SCHOOLS:

PLUGGING STUDENTS INTO NATURE THROUGH HABITAT

REHABILITATION ON SCHOOL GROUNDS ….....................142

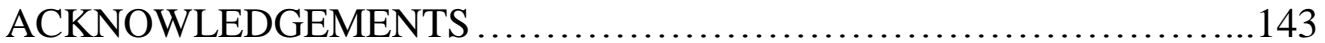

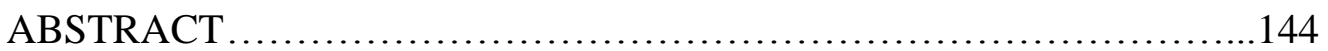

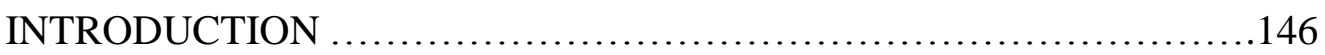

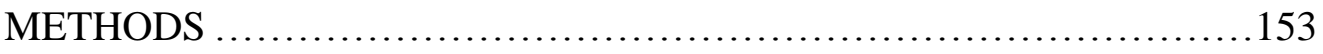

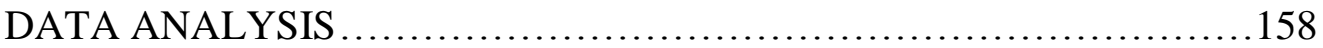

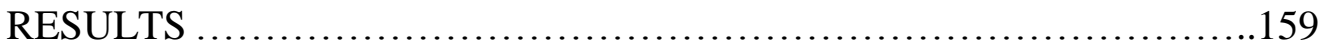

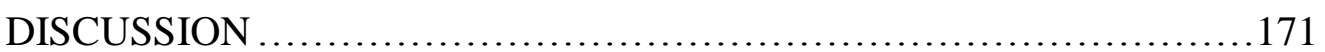

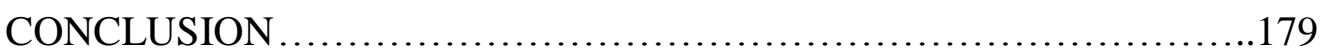

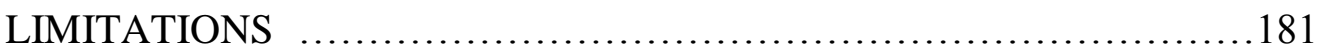

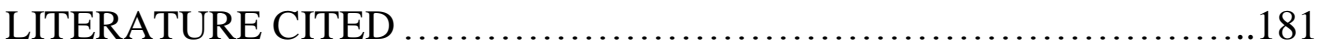

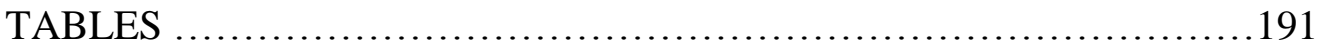

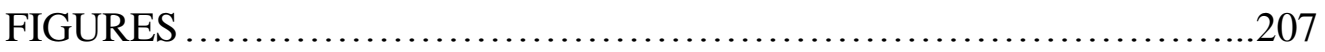

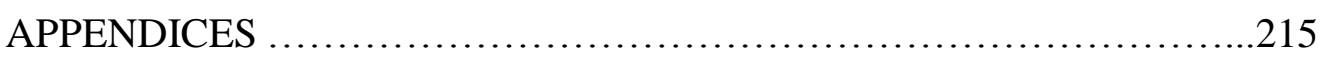

CONCLUSIONS AND FUTURE DIRECTIONS .........................220

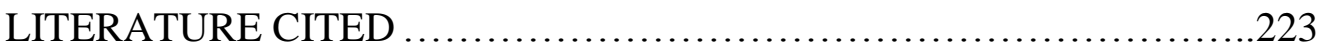

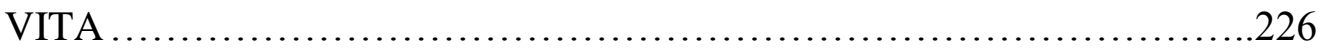




\section{LIST OF TABLES}

TABLE

PAGE

\section{CHAPTER I}

1.1 Number of plants including Torchwood, Wild Lime, and nectar plants planted at Elliott and Adams Keys. Numbers are shown per restoration area. Column on right shows total number of plants planted per restoration area

1.2 Plants planted in the butterfly gardens and FIU Nature Preserve. Note: MDC students were given plants to take home. Asterisks (*) denote exotic plants. See text (Field-site Description) for site abbreviations

1.3 Host plants planted in the gardens and Lepidoptera (butterflies, moths, and skippers) attracted to them. Asterisks denote exotic plants

1.4 Before garden construction, butterfly-species counts (pre-survey) were conducted (numbers indicated in parentheses) at each site. Several months later, a post-survey was conducted in the same area at each site. Asterisks (*) denote an increase in butterflies from the pre-survey. Note: Students at MDCHC did not construct a butterfly garden

\section{CHAPTER II}

2.1 Number and location of different plant species planted during the Schaus' Swallowtail Enhancement Project (Whelan and Atkinson, 2015). The majority of plants were sea torchwood, the primary host plant for the Schaus'

swallowtail butterfly

2.2 Sea torchwood and wild lime survivorship at different restoration sites in BNP. The average amount of days $( \pm \mathrm{SE})$ between measurements is listed in the table. $\mathrm{EKM}=$ Elliott Key Main Restoration Site, EKB = Elliott Key Breezeway Site, $\mathrm{AKM}=$ Adams Key Main Restoration Site, $\mathrm{AKB}=$ Adams Key Breezeway Site .73

2.3 Results from the Kruskal-Wallis test including a post-hoc pairwise comparisons analysis between sites. Three independent variables ( $\mathrm{CC}=$ Canopy Cover, Elev = Elevation, Soil Depth) were measured for sea torchwood and one independent variable (Elev $=$ Elevation) was measured for wild lime. TS $=$ Test Statistic, $\mathrm{SE}=$ Standard Error, Sig = Significance, EKM = Elliott Key Main Restoration Site, EKB = Elliott Key Breezeway Site, AKM = Adams Key Main Restoration Site, AKB = Adams Key Breezeway Site 
2.4 Independent variables $(\mathrm{CC}=$ Canopy Cover, Elev = Elevation, Soil Depth $)$ measured in the restored and breezeway sites at Elliott Key and Adams Key. Elevation was the only independent variable measured for wild lime. Median values were reported instead of average values because some data was not normally distributed for specific years and several sites. EKM = Elliott Key Main Restoration Site, EKB = Elliott Key Breezeway Site, AKM = Adams Key Main Restoration Site, AKB = Adams Key Breezeway Site ....

2.5 Results from the Kruskal-Wallis test including a post-hoc pairwise comparisons analysis between sites. Stem elongation (growth) was measured in 2013, 2014, and 2017 (see Table 2 for the number of growing days after the first measurement). Wild lime growth was measured in 2013 and 2014. TS = Test Statistic, $\mathrm{SE}=$ Standard Error, Sig = Significance, EKM = Elliott Key Main Restoration Site, EKB = Elliott Key Breezeway Site, AKM = Adams Key Main Restoration Site, $\mathrm{AKB}=$ Adams Key Breezeway Site

2.6 Stem elongation (growth) of wild lime and sea torchwood in the restored and breezeway sites at Elliott Key and Adams Key. Median values were reported instead of average values because growth data was not normally distributed for specific years and at several sites. EKM = Elliott Key Main Restoration Site, $\mathrm{EKB}=$ Elliott Key Breezeway Site, AKM = Adams Key Main Restoration Site, $\mathrm{AKB}=$ Adams Key Breezeway Site

2.7 Results from the stepwise linear regression analysis. Best fit models were assembled with the most relevant independent variables. Torch $=\mathrm{Sea}$ Torchwood, WL = Wild Lime

2.8 Results from the binary logistic regression analysis. Best fit models were assembled with the most relevant independent variables. $\mathrm{C} / \mathrm{S} \mathrm{r}^{2}=\mathrm{Cox}$ and Snell $r^{2}$, Nag $r^{2}=$ Nagelkerke $r^{2}, H \&$ L test $=$ Hosmer-Lemeshow test, Torch $=$ Sea torchwood, WL = Wild Lime

2.9 Receiver operating characteristic (ROC) curve results for sea torchwood and wild lime survivorship against increasing elevation and soil depth and decreasing canopy cover in restored and breezeway sites at Elliott Key and Adams Key. Accuracy of the ROC curve is measured by the area under the curve $(\mathrm{AUC} ; \mathrm{SE}=$ Standard Error). The AUC grade system: $>0.90-1.00=$ excellent $(\mathrm{A}),>0.80-0.90=\operatorname{good}(\mathrm{B}),>0.70-0.80=$ fair $(\mathrm{C}),>0.60-0.70=$

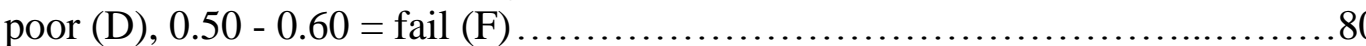

2.10 Projected subtropical dry forest habitat loss in Biscayne National Park (BNP) and Key Largo from imminent sea level rise (SLR). Dry forest habitat initially starts $0.30 \mathrm{~m}$ above sea level (95\% of dry forest) in BNP and Key Largo. Remaining dry forest habitat (in hectares) at each site is presented in SLR 
increments of $25 \mathrm{~cm}$ up to $200 \mathrm{~cm}$. SK = Sands Key, EK = Elliott Key, AK = Adams Key, ORK = Old Rhodes Key, TK = Totten Key, PKCR = Porgy Key and Caesar Rock, SK = Swan Key, BNP = Biscayne National Park, PABLK = Palo Alto Key, Broad Key, and Linderman Key, CLNWR = Crocodile Lake National Wildlife Refuge, KLHSBP = Key Largo Hammock State Botanical Site, UNP = Unprotected Dry Forest Habitat, KLSDF = Key Largo South Dry Forest, KLTF $=$ Key Largo Total Forest

\section{CHAPTER III}

3.1 Ants collected in pitfall traps at Elliott and Adams Keys: number of individuals of each species, and status

3.2 Ants collected in pitfall traps in the canopies of Amyris elemifera and Zanthoxylum fagara: number of individuals of each species, and status

3.3 Generalized linear model (negative binomial with log link) results of Pseudomyrmex gracilis collected on three different groups of trees

3.4 Results of predator exclusion experiment using $H$. cresphontes larvae on Amyris elemifera and Zanthoxylum fagara. Z scores (adjusted z-scores) were acquired through the contingency table analysis and then squared to obtain Chi square values....

3.5 Ant Danger Index (ADI) based on cumulative scores from: (1) ant species relative abundance, (2) time to discover caterpillar, (3) ability to recruit more ants, (4) early instar (small) mortality, and (5) late instar (large)

mortality

3.6 Ant Danger Index (ADI) based on cumulative scores from: (1) ant species relative abundance, (2) time to discover caterpillar, (3) ability to recruit more ants, (4) early instar (small) mortality, and (5) late instar (large) mortality

\section{CHAPTER IV}

4.1 Survey statements and interview questions used during the study. All students $(n=120)$ at each school (Air Base K-8 Center, North Hialeah Elementary, Whispering Pines Elementary) and treatment (experimental and control groups) were administered pre- and post-survey statements. A total of 48 students (16 students at each school; however, 8 students for each treatment) participated in pre- and post-interviews

4.2 Plants planted in the school butterfly gardens. Overall, 18-19 native plant species were distributed to Air Base K-8 Center (AB), North Hialeah 
Elementary (NHE), and Whispering Pines Elementary (WPE). Number of plants are reported below each school. The last column on the right depicts showy butterflies, moths, and skippers attracted to host plants planted in the school gardens (there are other less showy moth species, not listed). Some butterfly, moth, and skipper species are imperiled and unlikely to visit the school gardens; however, each plant added structural and functional value to the native plant butterfly garden

4.3 Science Teaching Efficacy Belief Instrument results from each school (AB = Air Base K-8 Center, NHE = North Hialeah Elementary, WPE $=$ Whispering Pines Elementary; Riggs and Enochs, 1990). SA = Strongly Agree, A = Agree, $\mathrm{UN}=$ Uncertain, $\mathrm{D}=$ Disagree, $\mathrm{SD}=$ Strongly Disagree. Results from 3 individual science teachers, one at each school.

4.4 Summary statistics of post-test scores (dependent variable) using an Analysis of Covariance (ANCOVA). The pre-test score was the covariate with the intervention as the independent variable. The intervention had a significant effect on post-test scores. Students in the experimental group significantly scored higher on the post-test in contrast to students in the control group (see Figures 4-7). Asterisk denotes significance

4.5 A total of $1205^{\text {th }}$ grade students attending three schools completed pre- and post-surveys. Sixty students were in experimental groups while the other 60 were in control groups. An exploratory factor analysis was performed and reduced 28 survey statements into 14 survey statements that loaded into 5 factors.

Quade's rank analysis of covariance was used to analyze survey results. Post-survey score was the dependent variable, pre-survey score was the covariate with the influence of gardens present at the place of residence before the study commenced, intervention of taking host and nectar plants home, and type of students' residence as independent variables. Type of residence was tested for all schools; however, it was not tested for individual schools due to small sample size

4.6 Results from the Likert scale pre- and post-surveys. Mean and standard deviations were reported displaying students' responses toward statements loaded in each factor. Bold indicates significant changes in students' attitude (see results in Table 5)

4.7 Results from pre- and post-interviews. The most prominent themes (> 20\% of respondents) inferred from interviews were displayed as decimals because of unequal respondents between students in the control and experimental groups. There were 24 respondents each (pre- and post-interviews) in the control group; however, 22 respondents conducted pre-interviews and 16 conducted post-interviews in the experimental group 
4.8 The number of home gardens reported by students at Air Base K-8 Center (AB), North Hialeah Elementary (NHE), and Whispering Pines Elementary (WPE). The experimental groups increased the number of home gardens; however, both groups equally increased the number of butterfly gardens.......206 


\section{LIST OF FIGURES}

FIGURE

PAGE

\section{CHAPTER I}

1.1 Current range of the Schaus' Swallowtail Butterfly in south Florida. Map generated by Helena Giannini............................................ 37

1.2 Study-site locations in Miami-Dade County (Biscayne National Park is outlined on the bottom right in the map). See text (Field-site Description) for site abbreviations

1.3 Tagged Zebra Longwing ready for release at the Florida International University nature preserve

1.4 Schaus' Swallowtail egg on Torchwood in the main restoration site at Elliott Key in Biscayne National Park (left); Giant Swallowtail caterpillar on Wild Lime in the main restoration site at Adams Key (right)

\section{CHAPTER II}

2.1 Schaus' swallowtail early instar caterpillar resting on sea torchwood in a subtropical dry forest habitat at Elliott Key in Biscayne National Park, Florida. Sea torchwood is the primary host plant for Schaus' swallowtail caterpillars (Minno and Emmel, 1993; Jameson, 2002)

2.2 The Keys that incorporate terrestrial landmass within the boundaries of Biscayne National Park (BNP) and North Key Largo in south Florida. The light-green color displays the extent of subtropical dry forest in BNP and North Key Largo. Sea torchwood, the primary host plant for the Schaus' swallowtail butterfly, predominantly grows in dry forest habitats (Lugo et al., 1978; Stalter et al., 1999; Jameson, 2002).

2.3 Restoration site locations, indicated with red boxes, of the Schaus' Swallowtail Habitat Enhancement Project in Biscayne National Park (BNP), Florida (left). The red lines outline the restoration sites at Elliott Key (top-right) and Adams Key (bottom-right) in BNP (Whelan, 2011)

2.4 Sea torchwood planted in the restoration site at Elliott Key in Biscayne National Park, Florida. Each plant received an aluminum tag to track survivorship and primary stem elongation. They also received mulch to minimize water loss from substrate desiccation. Leaf litter acts as a natural mulch in subtropical dry forest habitats (Ross et al., 2003) 
2.5 Elliott Key is a low-lying island within the boundaries of Biscayne National Park (BNP). Naturally occurring sea torchwood thrives at higher elevations within subtropical dry forest habitat at Elliott Key $(n=54, \overline{\mathrm{x}}=1.10 \mathrm{~m}, \mathrm{~s}=$ $0.28)$

2.6 Boxplots displaying canopy cover (\# of open squares) at each site. Canopy cover was recorded above each sea torchwood planted in the restored and breezeway sites at Elliott Key and Adams Key. Different letters above boxplots denote significance. Open circles represent suspected outliers; however, extreme outliers were removed. EK Main = Elliott Key Main Restoration Site, EK Breeze $=$ Elliott Key Breezeway Site, $\mathrm{AK}$ Main $=$ Adams Key Main Restoration Site, AK Breeze = Adams Key Breezeway Site

2.7 Boxplots displaying land elevation (m) at each site. Land elevation was recorded for each sea torchwood planted in the restored and breezeway sites. Elevation at each site was significantly different from other. Different letters above boxplots denote significance. Open circles represent suspected outliers; however, extreme outliers were removed. EK Main = Elliott Key Main Restoration Site, EK Breeze $=$ Elliott Key Breezeway Site, $\mathrm{AK}$ Main $=$ Adams Key Main Restoration Site, AK Breeze $=$ Adams Key Breezeway Site

2.8 Boxplots displaying soil depth $(\mathrm{cm})$ at each site. Soil depth was recorded at each sea torchwood planted in the restored and breezeway sites. Different letters above boxplots denote significance. Open circles represent suspected outliers; however, extreme outliers were removed. EK Main = Elliott Key Main Restoration Site, EK Breeze $=$ Elliott Key Breezeway Site, AK Main = Adams Key Main Restoration Site, AK Breeze = Adams Key Breezeway Site ...89

2.9 Box plots displaying sea torchwood stem elongation (growth in $\mathrm{cm}$ ) at each site during 2013. Different letters above boxplots denote significance. Open circles represent suspected outliers; however, extreme outliers were removed. EK Main $=$ Elliott Key Main Restoration Site, EK Breeze $=$ Elliott Key Breezeway Site, $\mathrm{AK}$ Main = Adams Key Main Restoration Site, AK Breeze = Adams Key Breezeway Site

2.10 Box plots displaying sea torchwood stem elongation (growth in $\mathrm{cm}$ ) at each site during 2014. Different letters above boxplots denote significance. Open circles represent suspected outliers; however, extreme outliers were removed. EK Main $=$ Elliott Key Main Restoration Site, EK Breeze $=$ Elliott Key Breezeway Site, $\mathrm{AK}$ Main = Adams Key Main Restoration Site, AK Breeze $=$ Adams Key Breezeway Site

2.11 Box plots displaying sea torchwood stem elongation (growth in $\mathrm{cm}$ ) at each site during 2017. Different letters above boxplots denote significance. Open circles represent suspected outliers; however, extreme outliers were removed. 
EK Main $=$ Elliott Key Main Restoration Site, EK Breeze $=$ Elliott Key Breezeway Site, AK Main = Adams Key Main Restoration Site, AK Breeze = Adams Key Breezeway Site

2.12 Boxplots displaying land elevation (m) at each site. Elevation was recorded for each wild lime planted in the restored and breezeway sites. Different letters above boxplots denote significance. EK Main = Elliott Key Main Restoration Site, EK Breeze = Elliott Key Breezeway Site, AK Main = Adams Key Main Restoration Site, AK Breeze $=$ Adams Key Breezeway Site

2.13 Boxplots displaying wild lime stem elongation (growth in $\mathrm{cm}$ ) at each site during 2013. Stem elongation was recorded for each wild lime planted in the restored and breezeway sites. Different letters above boxplots denote significance. Open circles represent suspected outliers; however, extreme outliers were removed. EK Main = Elliott Key Main Restoration Site, EK Breeze $=$ Elliott Key Breezeway Site, AK Main = Adams Key Main Restoration Site, AK Breeze = Adams Key Breezeway Site.

2.14 Boxplots displaying wild lime stem elongation (growth in $\mathrm{cm}$ ) at each site during 2014. Stem elongation was recorded for each wild lime planted in the restored and breezeway sites. Different letters above boxplots denote significance. Open circles represent suspected outliers; however, extreme outliers were removed. EK Main = Elliott Key Main Restoration Site, EK Breeze $=$ Elliott Key Breezeway Site, AK Main = Adams Key Main Restoration Site, AK Breeze = Adams Key Breezeway Site

2.15 The extent of available dry forest habitat at Elliott Key: (1) current sea level, (2) $1 \mathrm{~m} \mathrm{SLR}$, and (3) $2 \mathrm{~m} \mathrm{SLR}$

2.16 The extent of available dry forest habitat at Adams Key: (1) current sea level, (2) $1 \mathrm{~m} \mathrm{SLR}$, and (3) $2 \mathrm{~m} \mathrm{SLR}$

2.17 The extent of available dry forest habitat at Old Rhodes Key and Totten Key:

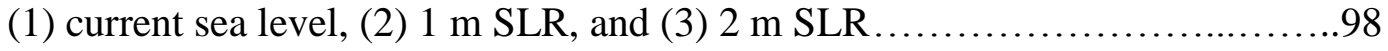

2.18 The extent of available dry forest habitat in North Key Largo: (1) current sea level, (2) $1 \mathrm{~m} \mathrm{SLR}$, and (3) $2 \mathrm{~m} \mathrm{SLR.}$

\section{CHAPTER III}

3.1 Geographic range of the federally endangered Heraclides aristodemus ponceanus in south Florida. The historic ranged is outlined in yellow, the current range is outlined in red, and the boundary of Biscayne National Park is outlined in blue. Map generated by Lydia Cuni 
3.2 Percentage of ant species captured in pitfall traps at Elliott and Adams Keys (tree canopy, trunk, and base). Overall, 1418 total ants comprising 25 ant species were captured and identified from pitfall traps

3.3 Percentage of ant species captured in pitfall traps on Amyris elemifera and Zanthoxylum fagara. Overall, 243 total ants comprising 12 ant species were captured and identified from pitfall traps used in the canopy. The four most common ants collected were $P$. gracilis, $C$. planatus, $C$. ashmeadi, and $C$. floridanus. They represented $87 \%$ of all collected ants on A. elemifera and $Z$. fagara. Pseudomyrmex gracilis and C. planatus are exotic species and represented $56 \%$ of the total sampled ants

3.4 Graphical representation of Pseudomyrmex gracilis collected on three different group of trees at Elliott and Adams Keys in Biscayne National Park, Florida. The bars represent averages of count data with standard error bars. Lowercase letters reflect statistical significance. Overall, P. gracilis was only significantly more frequent on Amyris elemifera than non-host trees. Amyris elemifera is the primary host tree for the Heraclides aristodemus ponceanus....137

3.5 The predator exclusion experiment involved 6 treatments, with physical barriers (tanglefoot and mesh cages) used in different combinations on Zanthoxylum fagara to compare caterpillar (early and late instar) survivorship against crawling and non-crawling predators. Survivorship was defined as persistence on leaf for 5 days. A contingency table analysis was performed. Asterisks denote significance in survivorship for the various treatments. Each treatment using early instar caterpillars had 16 trials, each treatment using late instar caterpillars had 15 trials. Refer to Table 4 for more details.

3.6 The average time $( \pm \mathrm{SE})$ the four most abundant ant species took to discover Heraclides cresphontes caterpillars placed on Amyris elemifera and Zanthoxylum fagara at Elliott Key, Biscayne National Park, Florida. A one-way ANOVA was performed followed by the Fisher's Least Significant Difference post-hoc test. Asterisk denotes significance

3.7 Ant recruitment to Heraclides cresphontes caterpillars placed on Amyris elemifera and Zanthoxylum fagara by the four most abundant ant species at Elliott Key, Biscayne National Park, Florida. Recruitment is displayed as the average number of ants attracted to caterpillars $( \pm \mathrm{SE})$. Ant recruitment allows ants to overwhelm larger prey such as late instar caterpillars ..................140

3.8 Early instar Heraclides aristodemus ponceanus caterpillar fends off an approach by Camponotus planatus. This approach is not aggressive; however, the $H$. a. ponceanus caterpillar does not tolerate contact by other organisms....141 


\section{CHAPTER IV}

4.1 Map of school locations and their proximity to natural forest communities and environmentally endangered lands (EEL) sites. $\mathrm{AB}=$ Air Base K-8 Center; $\mathrm{WPE}=$ Whispering Pines Elementary; NHE = North Hialeah Elementary .......207

4.2 Teacher and $5^{\text {th }}$ grade students working together to construct the butterfly garden at North Hialeah Elementary School. See table 2 regarding the number of plants and plant species added to their garden

4.3 Two students at Whispering Pines Elementary School are reading the identification guide titled, "Butterflies of Southeast Florida: A Guide to Common and Notable Species" (Minno, 2014)

4.4 Combining all schools (mean $\pm \mathrm{SE}$ ), there was a significant increase in posttest scores for the experimental group; however, no significant increase for the control group (paired sample $t$-tests, experimental group: $d f=59, t=-6.301$, $\mathrm{p}<0.001$; control group: $d f=59, t=-1.239, \mathrm{p}=0.220)$. The intervention had a significant effect on post-test scores (ANCOVA; $d f=1 ; F=25.771$, $\mathrm{p}<0.001)$

4.5 At Air Base K-8 Center (mean $\pm \mathrm{SE}$ ), there was a significant increase on posttest scores for the experimental group; however, no significant increase for the control group (paired sample $t$-tests; experimental group: $d f=20, t=-3.871$, $\mathrm{p}<0.001$; control group: $d f=19, t=-0.937, p=0.360)$. The intervention had a significant effect on post-test scores (ANCOVA; $d f=1 ; F=9.926$, $\mathrm{p}=0.003)$

4.6 At North Hialeah Elementary School (mean \pm SE), there was a significant increase on posttest scores for the experimental group; however, no significant increase for the control group (paired sample $t$-tests; experimental group: $d f=$ $17, t=-2.403, p=0.028$, control group: $d f=19, t=-0.573, p=0.573)$. The intervention had a significant effect on post-test scores (ANCOVA; $d f=1$; $F=6.203, \mathrm{p}=0.018)$

4.7 At Whispering Pines Elementary School (mean \pm SE), there was a significant increase on posttest scores for the experimental group; however, no significant increase for the control group (paired sample $t$-tests; experimental group: $d f=$ $20, t=-4.705, p<0.001$, control group: $d f=19, t=-0.701, \mathrm{p}=0.492)$. The intervention had a significant effect on post-test scores (ANCOVA; $d f=1$; $F=10.986, \mathrm{p}=0.002$ ) 
4.8 Boxplots depicting post-survey results for the five factors between the experimental and control groups (Scale: strongly agree $=5$, agree $=4$, neutral $=3$, disagree $=2$, strongly disagree $=1$ ) 


\section{LIST OF APPENDICES}

APPENDIX

PAGE

\section{CHAPTER I}

1.1 Latin names, taxonomic authority, and common names of butterfly, moth, and skipper species mentioned in this paper...................................41

1.2 Latin names, taxonomic authority, and common names of plants mentioned in

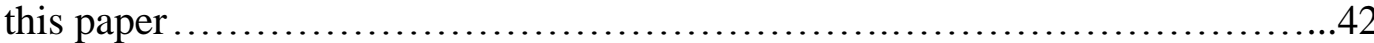

\section{CHAPTER IV}

4.1 Map of south Florida depicting the historic (outlined in green) and current range (outlined in red) of the federally endangered Schaus' swallowtail butterfly (Heraclides aristodemus ponceanus). The largest populations exist on islands in Biscayne National Park (outlined in blue). This map was enlarged (5' x 3') for the activity titled, "Place the Schaus' swallowtail butterfly in the right habitat". This activity involved participation from the entire class. One blind-folded student was selected and placed at a random location in the classroom. The objective was to navigate the magnetic Schaus' swallowtail butterfly to suitable habitat (outlined in red) on the large map. Classmates were not allowed to verbally communicate to the blind-folded student; they were only able to navigate the blind-folded student using different toy instruments. Classmates were given 5-7 minutes to brainstorm and execute their strategic plan (Clayborn et al., 2017)

4.2 Data observation sheet used by each student in the experimental treatment group. Data sheets were double-sided with detailed instructions on the back page. Parents and students were encouraged to report their findings using Twitter handles customized for each school 


\section{INTRODUCTION}

South Florida hosts numerous species of butterflies, where temperate and tropical species coexist in various habitats, ranging from residential neighborhoods to subtropical dry forests. Tropical dry forests are dominated by broad-leaved trees concentrated in the southern portion of Florida (Snyder et al., 1990; Ross et al., 1992). The imperiled Schaus' swallowtail butterfly (Heraclides aristodemus ponceanus) inhabits dry forest ecosystems in south Florida (Salvato, 2008) and, over time, have experienced significant population reductions (Loftus and Kushlan, 1984; Calhoun et al., 2002). This large butterfly, endemic to south Florida and the Bahamas, was among the first insects given federal protection; it was listed as threatened in 1976, and then reclassified as endangered in 1984 (Smith et al., 1994; Bibb and Hughes, 2007). Historically, the Schaus' swallowtail butterfly occupied subtropical dry forests in both peninsular south Florida and the Florida Keys, but now is restricted to several islands in the Florida Keys (Emmel et al., 1988). Many butterflies including federally and state-listed species inhabit subtropical dry forests in Florida. All dry forest ecosystems are federally protected in Biscayne National Park (BNP) while more than half is state protected in Key Largo (Karim and Main, 2009). In BNP, Elliott Key harbors the largest population of Schaus' swallowtail butterflies; however, the population is not uniformly distributed throughout the island. Schaus' swallowtail butterflies also occur on other islands within the boundaries of BNP and North Key Largo (Emmel et al., 1988; Daniels, 2014; Minno, 2015).

A habitat restoration project was designed to restore degraded habitat within prime Schaus' swallowtail butterfly territory by planting and nurturing $>3,000$ host and nectars plants, mainly sea torchwood (Whelan, 2011). The Schaus' Swallowtail Habitat 
Enhancement Project was dependent on many volunteers dedicated to habitat and insect conservation. Applied citizen-science fieldwork connects people to nature and butterflies. The process of physically planting sea torchwood in the dry forest ecosystem vital to the Schaus' swallowtail butterfly's survival presents opportunities for volunteers and staff to engage in environmental stewardship and conservation in a national park. A curriculum unit devoted to the Schaus' swallowtail butterfly, insect conservation, and native plant butterfly gardens was implemented at schools in Miami-Dade County to connect children and adults to the plight of the Schaus' swallowtail butterfly in BNP, participate in habitat rehabilitation (native plant butterfly gardening) on school grounds, and conduct local butterfly surveys (Clayborn et al., 2017).

The expansion of suitable butterfly habitat on south Florida's mainland could mitigate the eventual loss of viable habitat in the Florida Keys (Ross et al., 2009; Minteer and Collins, 2010; Maschinski et al., 2011). Sea torchwood and wild lime planted in the restoration sites at Elliott Key and Adams Key were monitored for survivorship and growth from 2012 to 2017. Specific environmental variables including canopy cover, elevation, and relative soil depth were measured adjacent or above the sea torchwood and wild lime plants to determine their contribution to survivorship and growth. The imminent threat of sea level rise (SLR) will negatively impact dry forest ecosystems where sea torchwood, wild lime, and the Schaus' swallowtail butterfly exists. Spatial analyses of dry forest landscapes in BNP and Key Largo against the onslaught of rising seas can provide useful information depicting estimates of dry forest habitat loss based on incremental SLR. Each island has a varying elevational gradient with different amounts of available dry forest habitat (Karim and Main, 2009). In the future, some islands might 
be more robust against SLR and continue to support Schaus' swallowtail butterfly populations.

Dry forest habitat loss because of SLR is one of many threats to the Schaus' swallowtail butterfly. Exotic ants are potentially notorious agents in the decline of the Schaus' swallowtail butterflies and other rare butterflies. Red imported fire ants (Solenopsis invicta) are considered a threat to rare butterflies because of their highly predatory nature (Forys et al., 2001). Another exotic ant, the elongate twig ant (Pseudomyrmex gracilis), is an arboreal ant that poses potential risk to rare butterflies as they actively search for prey on shrubs and trees (Saarinen and Daniels, 2006; Wetterer, 2010). The restored sites within Elliott Key and Adams Key are exposed with minimal canopy cover and numerous small saplings. This habitat structural shift invites ants that prefer disturbed habitats (King and Tschinkel, 2006; Didham et al., 2007). The Schaus' swallowtail butterfly's limited range and exposure to natural extreme weather events, may work in concert with greater predation on immature stages to reduce butterfly numbers (Schoener et al., 2001). Quantitative research is necessary to assess potential risks imposed by exotic ants to corroborate anecdotal observations (Forys et al., 2002).

The sustainable conservation of dry forest ecosystems and preservation of imperiled flora and fauna needs advocates from peripheral and at large communities. The Schaus' swallowtail butterfly can be used as a flagship species to explain the plight of imperiled butterflies and conservation goals in south Florida. A sense of ownership, awareness, and inclusion in decision-making processes are essential to the protection of these rare species vulnerable to extinction (Guiney and Oberhauser, 2009; Grunova et al., 2017). Active engagement and participation through service-learning projects and classroom activities 
encourage youth to explore their outdoor surroundings and learn about ecosystems on real-time. We live in a present time where experiential learning and stories about different species matter because how we feel about and connect with those species will determine their survival in the present and future (Grajal et al., 2017).

Schools provide an opportunity to restore depauperate green space in the local community. Schoolyards can provide teachers and students not only a way to increase the biodiversity of organisms, but learning and empathy for nature as well (Lieberman and Hoody, 1998). An integrated insect curriculum unit with an emphasis on the Schaus' swallowtail butterfly was implemented at three schools in Miami-Dade County. A quasiexperimental mixed methods approach (pre- and post-tests, pre- and post-surveys, preand post-interviews) was applied to quantify students' gained knowledge, attitudinal shifts, and additional butterfly gardens in neighboring communities (Cook and Campbell, 1979; Tashakkori and Teddlie, 1998). Such outdoor experiences may encourage further activities by families and lifelong compassion for the environment. Ongoing exposure to school gardens through maintenance and class assignments can help students achieve academically, become socially aware about environmental quality, and create social capital where students work for the collective good of their community.

In order to build on current knowledge about the Schaus' swallowtail butterfly and connect with schools and local communities, I undertook a 4-pronged study that investigated citizen science and environmental stewardship, host plant survivorship and growth, threats from rising seas and predators, and measured community advocacy towards south Florida insect conservation. 
The objectives of this dissertation were:

1) To restore subtropical dry forest in Biscayne National Park (BNP) for the federally endangered Schaus' swallowtail butterfly;

2) To assess torchwood and wild lime survivorship and growth in the restorations sites (BNP) and project dry forest habitat loss because of imminent sea level rise;

3) To quantify ant activity and diversity and record invertebrate predator-prey interactions against Heraclides caterpillars in dry forests in BNP; and

4) To implement a south Florida butterfly conservation curriculum unit at schools in Miami-Dade County, and to evaluate students' gained knowledge and attitudes toward south Florida environments, insects and butterflies, and conservation.

Some of the chapters of this dissertation have been published in peer-reviewed scientific journals, and the others are either in review or formatted for submission. Chapter 1 was published in the journal of Southeastern Naturalist (Clayborn et al. 2017. SENA 16(10): 26-46); Chapter 2 was submitted to the journal of Biological Conservation; Chapter 3 was published in the journal of Insect Conservation (Clayborn and Koptur. 2017. J Insect Conserv 21(4): 689-702); and Chapter 4 was submitted to the journal of the Learning Sciences.

\section{LITERATURE CITED}

Bibb, K., and Hughes, P. 2007. United States Fish and Wildlife Service Schaus Swallowtail butterfly (Heraclides aristodemus ponceanus) 5-Year review: Summary and evaluation. United States Fish and Wildlife Service, Southeast Region, South Florida Ecological Services Office, Vero Beach, Florida. Available online at http://ecos.fws.gov/docs/five_year_review/doc1983.pdf. Accessed 20 February 2017. 
Calhoun, J.V., Slotten, J.R., and Salvato, M.H. 2002. The rise and fall of tropical blues in Florida: Cyclargus ammon and Cyclargus thomasi bethunebakeri (Lepidoptera: Lycaenidae). Holarctic Lepidoptera, 7: 13-20.

Clayborn, J., Koptur, S., O’Brien, G., and Whelan, K.R.T. 2017. The Schaus swallowtail habitat enhancement project: An applied service-learning project continuum from Biscayne National Park to Miami-Dade County public schools. Southeastern Naturalist, 16(10): 26-46.

Cook, T.D., and Campbell, D.T. 1979. Quasi-experimentation: Design \& analysis issues for field settings. Houghton Mifflin, Boston.

Daniels, J.C. 2014. Occurrence, distribution and ecological studies of the endangered Schaus' Swallowtail butterfly. A report to the U.S. Fish and Wildlife Service and Imperiled Butterflies of South Florida Workgroup. 24 pp.

Didham, R.K., Tylianakis, J.M., Gemmell, N.J., Rand, T.A., and Ewers, R.M. 2007. Interactive effects of habitat modification and species invasion on native species decline. Trends in Ecology \& Evolution, 22: 489-496.

Emmel, T.C., Minno, M.C., Nation, J.L. Jr, Eliazar, P.J., Cotter, A.J., Bustamante, E.G., Ritland, D.B., and Goode, M.R. 1988. Habitat requirements and status of the endemic Schaus' swallowtail in the Florida Keys. Final Project Report, Project Number GFC86-023. Submitted to Florida Game and Fresh Water Fish Commission Division of Wildlife Nongame Wildlife Section, Tallahassee, Florida.

Forys, E.A., Quistorff, A., and Allen, C.R. 2001. Potential fire ant (Hymenoptera: Formicidae) impact on the endangered Schaus swallowtail (Lepidoptera: Papilionidae). Florida Entomologist, 84: 254-258.

Forys, E.A., Allen, C.R., and Wojcik, D.P. 2002. Influence of the proximity and amount of human development and roads on the occurrence of the red imported fire ant in the lower Florida Keys. Biological Conservation, 108: 27-33.

Grajal, A., Luebke, J.F., Kelly, L.D., Matiasek, J., Clayton, S., Karazsia, B.T., Saunders, C.D., Goldman, S.R., Mann, M.E., and Stanoss, R. 2017. The complex relationship between personal sense of connection to animals and self-reported proenvironmental behaviors by zoo visitors. Conservation Biology, 31(2): 322-330.

Grunova, M., Brandlova, K., Svitalek, J., and Hejcmanova, P. 2017. Environmental education supports conservation action by increasing the immediate and long-term environmental knowledge of children in West Africa. Applied Environmental Education and Communication, 16(1): 3-16. 
Guiney, M.S., and Oberhauser, K.S. 2009. Insects as flagship conservation species. Terrestrial Arthropod Reviews, 1(2): 111-123.

Karim, A., and Main, M.B. 2009. Habitat fragmentation and conservation strategies for a rare forest habitat in the Florida Keys archipelago. Urban ecosystems, 12(3): 359-370.

King, J.R., and Tschinkel, W.R. 2006. Experimental evidence that the introduced fire ant, Solenopsis invicta, does not competitively suppress co-occurring ants in a disturbed habitat. Journal of Animal Ecology, 75: 1370-1378.

Lieberman, G.A. and Hoody, L.L. 1998. Closing the Achievement Gap: Using the Environment as an Integrating Context for Learning. San Diego, CA: State Environment and Education Roundtable.

Loftus, W., and J. Kushlan. 1984. Population fluctuations of the Schaus swallowtail (Lepidoptera: Papilionidae) on the islands of Biscayne Bay, Florida, with comments on the Bahaman swallowtail. Florida Entomologist, 67: 277-287.

Maschinski, J., Ross, M.S., Liu, H., O'Brien, J., von Wettberg, E.J., and Haskins, K.E. 2011. Sinking ships: conservation options for endemic taxa threatened by sea level rise. Climatic Change, 107: 147-167.

Minno, M.C. 2015. 2015 Schaus' swallowtail (Heraclides aristodemus ponceanus) monitoring on Key Largo, Florida. Southern Lepidopterists' News, 37(3): 106-107.

Minteer, B.A., and Collins, J.P. 2010. Move it or lose it? The ecological ethics of relocating species under climate change. Ecological Applications, 20(7): 1801-1804.

Ross, M.S., O'Brien, J.J., and Flynn, L.J. 1992. Ecological site classification of Florida Keys terrestrial habitats. Biotropica, 24(4): 488-502.

Ross, M.S., J.J. O'Brien, R.G. Ford, K. Zhang, and A. Morkill. 2009. Disturbance and the rising tide: the challenge of biodiversity management on low-island ecosystems. Frontiers in Ecology and the Environment 7(9): 471-478.

Saarinen, E.V., and Daniels, J.C. 2006. Miami blue butterfly larvae (Lepidoptera: Lycaenidae) and ants (Hymenoptera: Formicidae): New information on the symbionts of an endangered taxon. Florida Entomologist, 89: 69-74.

Salvato, M. 2008. Investigator's annual report to Biscayne National Park: Annual butterfly survey on Elliott Key for the North American Butterfly Association. Fish and Wildlife Service, Vero Beach, FL.

Schoener, T.W., Spiller, D.A., and Losos, J.B. 2001. Predators increase the risk of catastrophic extinction of prey populations. Nature, 412: 183-186. 
Smith, D.S., Miller, L.D., Miller, J.Y., and Lewington, R. 1994. The butterflies of the West Indies and south Florida. Oxford University Press, New York. 264 pp.

Snyder J.R., Herndon A., and Robertson W.B.J. 1990. South Florida Rockland. In: Myers R.L., Ewel J.J. (eds) Ecosystems of Florida. University Press of Florida, Florida, pp 230-277.

Tashakkori, A., and Teddlie, C. 1998. Mixed methodology: Combining qualitative and quantitative approaches. Sage, Vol. 46, Thousand Oaks, California.

Wetterer, J.K. 2010. Worldwide spread of the graceful twig ant, Pseudomyrmex gracilis (Hymenoptera: Formicidae). Florida Entomologist 93: 535-540.

Whelan, K.R.T. 2011. Schaus Swallowtail butterfly (Heraclides aristodemus ponceanus) habitat enhancement in Biscayne National Park. Project Report. Funded by FWS South Florida Coastal Program. National Park Service, Palmetto Bay, FL. 9 pp. 


\section{CHAPTER I}

THE SCHAUS' SWALLOWTAIL HABITAT ENHANCEMENT PROJECT: AN APPLIED SERVICE-LEARNING PROJECT CONTINUUM FROM BISCAYNE NATIONAL PARK TO MIAMI-DADE COUNTY PUBLIC SCHOOLS

Published in Southeastern Naturalist

Clayborn, J., Koptur, S., O’Brien, G., and Whelan, K.R.T. 2017. The Schaus swallowtail habitat enhancement project: An applied service-learning project continuum from Biscayne National Park to Miami-Dade County public schools. Southeastern Naturalist, 16(10): 26-46. 


\title{
ACKNOWLEDGEMENTS
}

We thank S. Zona, Y. Reynaldo, and the editors and anonymous reviewers for helpful suggestions on the manuscript; Biscayne National Park and National Park Service personnel for providing access to the study sites (study numbers BISC-00041 and BISC00046 under permits BISC-2014-SCI-0031, BISC-2015-SCI-0023, and BISC-2016-SCI0044), and their continued commitment to protecting the jewels in the National Parks. We are grateful to all volunteers from the Sierra Club, Florida International University, University of Miami, Doral High School, John Pennekamp State Park, Biscayne National Park, and the National Park Service for their assistance in this project; and the faculty and teachers at Miami-Dade County schools and FIU for improving the Schaus and Coastal Hardwood Hammocks curriculum unit. This work was supported by the US Fish and Wildlife Service, and the FWS South Florida Coastal Program provided partial funding. This paper is contribution \# 326 to the Tropical Biology Program at Florida International University, Miami, FL.

\begin{abstract}
Urbanization in Miami-Dade County has modified large tracts of suitable habitats into smaller patches and increased distance between habitats. As a result, the endangered Heraclides aristodemus ponceanus (Schaus' swallowtail), which historically inhabited subtropical dry forests in south Florida and the Florida Keys, is now restricted to several islands in the Florida Keys and its numbers are precipitously declining. Here we report on a project that combined a remote in situ restoration project with a community outreach component that brought the restoration effort to local urban elementary schools. The
\end{abstract}


Schaus' Swallowtail Habitat Enhancement Project in Biscayne National Park utilized volunteers to remove exotic plants and plant over 3,000 Amryis elemifera (sea torchwood) and Zanthoxylum fagara (wild lime), which are host plants for the Schaus' swallowtail. After planting and initial establishment, we monitored growth and survival of host plants. We developed the Schaus and Coastal Hardwood Hammock curriculum unit in partnership with teachers and university faculty, and implemented it at 8 locations including 5 public schools in an ex situ outreach and education program. Lesson plans aimed to: (1) inform students about the Schaus' swallowtail, (2) increase the number and size of native-plant butterfly gardens at schools and homes, and (3) thwart "extinction of experience" in nature for school children. Teachers implement applied activities modeled on the habitat enhancement project in Biscayne National Park in the classroom and on school grounds, and used them to illustrate the butterfly life cycle, species' niche requirements, biodiversity, and restoration and conservation of south Florida habitats. Ongoing engagement with school gardens through maintenance and project-based assignments can help students achieve academically and become responsible environmental stewards.

\section{INTRODUCTION}

Charismatic, "flagship" species such as butterflies have been used to raise awareness and promote conservation and biodiversity of organisms, ecosystems, and resources in the US and other countries (Leader-Williams and Dublin 2000, Walpole and LeaderWilliams 2002). South Florida hosts over 100 species of butterflies; temperate and tropical species coexist in various habitats, ranging from residential neighborhoods to 
subtropical dry forests (Minno and Emmel 1993). Urbanization in south Florida has reduced large tracts of viable habitats into smaller patches (Alonso and Heinen 2011). Habitat loss is a major factor that has contributed to the decline of insect species globally (Bender et al. 1998, Ricketts 2001, Ricketts et al. 2008, Taki and Kevan 2007). Anthropogenic development has modified the natural environment through habitat simplification, expanded matrix (unsuitable surrounding habitat), and increased distance between viable habitats (Rosa et al. 2004). As a result, many insect species, often habitat specialists, have declined to very low numbers.

Many butterfly species have experienced significant population reductions (Calhoun et al. 2002, Loftus and Kushlan 1984, USFWS 2008), including Heraclides aristodemus ponceanus (Schaus' Swallowtail), a species symbolic of the plight of many insect species in south Florida. Historically, the Schaus' Swallowtail inhabited subtropical dry forests in both peninsular south Florida and the Florida Keys, but it is now restricted to several islands in the Florida Keys (Fig. 1). The Schaus' Swallowtail, endemic to south Florida and the Bahamas, was among the first insects given federal protection; listed as threatened in 1976, it was reclassified as endangered in 1984 (Smith et al. 1994; USFWS 2008, 2015). Many butterflies, including federally and state-listed species, inhabit subtropical dry forests (known as hardwood hammocks in Florida; Snyder et al. 1990). Hardwood hammocks in the northern Florida Keys are vital to the Schaus' Swallowtail because the preferred host plant, Amyris elemifera (Torchwood), exists primarily in this particular habitat (Minno and Emmel 1993, Rutkowski 1971). Torchwoods are subcanopy trees that thrive in gaps in the interior and along the edges of hardwood hammocks (Jameson 2002, Ray et al. 1998, Ross et al. 2001). Hardwood hammocks in 
the Florida Keys experienced timber harvests from the 1700s until the 20th century, followed by agricultural cultivation (early 1900s), and a transition to human habitation and tourism uses (Snyder et al. 1990, Strong and Bancroft 1994). Development and tourism have had direct and indirect deleterious effects on local flora and fauna. Direct effects include loss of land area, habitat degradation, and pollution; indirect effects include the spread of invasive species and the impacts of mosquito abatement treatments (Hoekstra et al. 2005, Janzen 1988, Roe et al. 1997). As a result, some native species in the Florida Keys are now imperiled or extinct.

The Schaus' Swallowtail Habitat Enhancement Project in Biscayne National Park (BNP) is an ongoing project on Elliott and Adams Keys - 2 islands protected within the boundaries of BNP — since 2011. National parks can provide refugia for imperiled insects to thrive and flourish; the protection of natural habitats and restoration of degraded areas are 2 of many solutions available to protect Earth's biodiversity (Hoekstra et al. 2005, Oliver et al. 2010). Hardwood hammocks are protected in BNP from both development and mosquito abatement. Biscayne National Park is home to imperiled species extirpated from the Floridian mainland, and continues to protect the Schaus' Swallowtail from deleterious, anthropogenic impacts. The project was designed by National Park Service (NPS) staff to enhance the habitat of the endangered butterfly. They planned to restore degraded habitat overgrown with invasive plant species within the butterfly's prime territory by planting and nurturing large numbers of host plants, mainly Torchwood (Whelan 2011). The distribution of larval host plants is clustered (Jameson 2002, Whelan 2011); therefore, another goal of the project was to more widely distribute larval host plants across the landscape to help increase overall butterfly population stability, by 
mitigating against negative stochastic episodes that might impact the current limited population of larval host plants (Whelan 2011).

We also sought to raise public awareness of the importance of these natural areas in the conservation of threatened and imperiled species, and instill stewardship in project volunteers. Information explaining the human and natural history of south Florida, especially with regard to hardwood hammocks and the Schaus' Swallowtail, are readily available at the BNP nature center, and are also featured on the interpretive trail at Elliott Key. The decrease in visitors to this and other parks is symptomatic of the disconnection between humans and nature which has increased over time in south Florida (Pergams and Zaradic 2008, Zaradic and Pergams 2007). Despite an increase in "baby boomer" visitors, overall visitation to national parks has steadily declined over the years. The low attendance rates among young people foreshadow an uncertain future for the conservation and preservation of natural resources (NPS 2015; Pergams and Zaradic 2006, 2008; Stevens et al. 2014). People who are introduced to natural areas as children are more likely to value them as adults (Bögeholz 2006, Duda et al. 1998, Hungerford and Volk 1990, Louv 2008, Pergams and Zaradic 2008, Wells and Lekies 2006). When children have repeated exposure to nature, they often grow up to be adults that advocate for the protection of natural areas and biodiversity conservation (Chawla 1998, Hungerford and Volk 1990, Matthews and Riley 1995, Wells and Lekies 2006).

The Schaus' Swallowtail Habitat Enhancement Project in BNP has involved volunteers since 2011 to help restore patches of land by removing widespread exotic, invasive vegetation and replanting native butterfly-attracting plants (Whelan and Atkinson 2015). A similar model can be applied in local communities (Mathew and Anto 
2007); schools, community centers, businesses, and residences can enhance habitat by constructing butterfly gardens with a focus on native plants (Vickery 1995). These places provide opportunities to expose legions of young people to the national parks through in situ field trips to natural areas and ex situ activities in the classroom or schoolyard, with activities ranging from exploratory observations to service-learning projects. Based on the Schaus' Swallowtail Habitat Enhancement Project in BNP, J. Clayborn designed the Schaus and Coastal Hardwood Hammock curriculum unit (containing multiple lesson plans) to connect students in Miami-Dade County to their local environment in south Florida. Participating professors and veteran teachers from Air Base K-8 Center, Coral Terrace Elementary, Florida International University, and Gateway Environmental K-8 Learning Center provided comments to improve the initial draft. The curriculum uses the charismatic Schaus' Swallowtail as a flagship species to demonstrate habitat restoration, a process which can also be applied locally at schools and homes to collectively benefit other butterfly and invertebrate species. Many students were unaware that south Florida's remnant ecosystems were historically expansive and exist now only as fragments outside of BNP, Big Cypress National Preserve, and Everglades National Park (Myers and Ewel 1990). This disconnect in understanding of the significance of native ecosystems can be detrimental to many organisms if anthropogenic constructs replace natural ecosystems.

As part of the newly developed curriculum, schoolyard gardens were created in most of the schools involved; most students active in this ex situ outreach and educational program did not participate in the Schaus' Swallowtail Habitat Enhancement Project in BNP. Enhancing areas such as schools and community centers by planting native plants, removing invasive plants, and minimizing pesticide application can provide suitable 
habitats that protect butterflies (including imperiled taxa) and other species (especially arthropods) from urbanization (Ricketts 2001). Host plants are critical for all butterflies to maintain their populations (Dennis et al. 2004, Minno and Emmel 1993, Vickery 1995); adult butterflies are less abundant in areas lacking such plants (Mathew and Anto 2007). Outreach programs that integrate habitat restoration projects with habitat rehabilitation projects in backyards, parks, and schoolyards can simultaneously help thwart extinction of species of imperiled butterflies in south Florida and "extinction of experience" in nature for children in urban areas (Louv 2008, Miller 2005, Pyle 1978).

The project at BNP and those carried out in other communities had the following objectives:

\section{$\underline{\text { Schaus' Swallowtail Habitat Enhancement Project }}$}

- Establish supplemental plantings of host species Torchwood and Zanthoxylum fagara (Wild Lime) in various locations at BNP.

- Restore critical hardwood hammock habitat by removing invasive plant species and planting native species.

- Provide volunteer-based in situ outreach and education programs to increase public awareness of Schaus' Swallowtail habitat and instill stewardship values.

$\underline{\text { Schaus and Coastal Hardwood Hammock curriculum unit }}$

- Replicate the Schaus' Swallowtail Habitat Enhancement Project in the Schaus and Coastal Hardwood Hammock curriculum unit created for urban schools and communities in south Florida. 
- Construct native plant butterfly gardens with high plant diversity, and observe and identify different butterfly species; in some cases, track the movement of butterflies from school grounds to adjacent communities.

- Implement an ex situ education and outreach program to promote the use of native plants and awareness of the negative implications of pesticide application for butterflies.

Note that complete lists of all species mentioned in this paper including the authorities and common names are provided in Appendices 1 and 2.

\section{FIELD-SITE DESCRIPTION}

Originally established as Biscayne National Monument in 1968, BNP became a national park in 1980. There is limited terrestrial area in BNP; $95 \%$ of the park is comprised of marine environments. The terrestrial area consists of a narrow strip of land on the mainland and 42 islands, the majority of which are dominated by mangrove forest (2400 ha).

Hardwood hammocks are dense, evergreen, subtropical dry forests dominated by broad-leaved trees that occur primarily in the southern portion of Florida (Ross et al. 1992, Snyder et al. 1990). Hardwood hammocks of the northern and middle Keys have marine-based limestone outcroppings (Key Largo limestone), which are partially covered with a shallow layer of endogenous soil (Armentano et al. 2002, Horvitz and Koop 2001). Coastal hardwood hammocks in BNP cover about 723 ha (23\%) of the terrestrial lands (Whelan et al. 2013). The largest island, Elliott Key, is roughly 7 miles long and nearly a mile wide; the hardwood hammock covers $68 \%$ of its area and $43 \%$ of Adams Key 
(Whelan et al. 2013). Many of these keys are currently impacted by exotic, invasive plant and animal species (FISP 2017, Invasive.org 2017). Approximately 500,000 people visit BNP each year (NPS 2015).

For this study, we established 2 site locations in BNP: Adams Key (AK) and Elliott Key (EK) (Fig. 2). Other parts of this research were conducted at Florida International University's (FIU) nature preserve, greenhouse, and the Ziff Education Building; the Miami-Dade College Hialeah Campus (MDC); and the Open House Ministries Community Center (OHM). We also had sites at 5 public schools: Air Base $\mathrm{K}-8$ Center (AB), Coral Terrace Elementary (CTE), Gateway Environmental K-8 Learning Center (GEL), North Hialeah Elementary School (NHE), and Whispering Pines Elementary School (WPE) (Fig. 2).

\section{METHODS}

Establishment of Torchwood corridors in Biscayne National Park, Elliott Key, and

\section{Adams Key}

The enhancement project involved planting Torchwood in restoration areas, and along an 8.05-km (5-mile) north-south trail down the middle of Elliott Key to provide a corridor of host plants (2 ha [4.94 ac]) between known Schaus' Swallowtail locations and restoration areas. The 2 restoration areas were initially dominated by 2 invasive plant species, Colubrina asiatica (Latherleaf) and Neyraudia reynaudiana (Burma Reed), which became established after disturbances at the sites. Enhancement project collaborators removed invasive plants and replaced them with_native vegetation at 2 sites: 1 on Elliott Key (0.58 ha [1.43 ac]) and 1 on Adams Key (0.27[0.66 ac]). The 
establishment of native plants can prevent, or substantially_reduce, the reestablishment of invasive species (Berger 1993, Egan and Howell 2001). Numerous Torchwood and Wild Lime seedlings (host plants for Schaus' Swallowtail), as well as other species of typical hardwoods, were planted to allow development of upper-canopy vegetation in the restoration area.

\section{Establishment of Supplemental Host Plants}

National Park Service (NPS) staff, John Pennekamp State Park staff (Key Largo, FL), volunteers, and a commercial nursery operator collected Torchwood and Wild Lime fruits from native populations. The fruits were transported to a native plant nursery in Homestead, FL, in 2011-2013, where the globose Torchwood berries were processed by removing the fleshy outer coat, scarifying the seed, and planting them in germination trays. Project personnel removed Wild Lime seeds from the follicles, and placed single seeds directly into germination trays, and grew the resulting seedlings in a greenhouse for a year before transporting them to Elliott and Adams Keys for planting.

J. Clayborn gave volunteers a brief lecture about insect conservation and the plight of the Schaus' Swallowtail before they began fieldwork. Volunteers and other project personnel proceeded to the field for hands-on service-learning work in the restoration areas. Their tasks included digging, planting, watering, and tracking host-plant growth and survival at both Elliott and Adams Keys. Upon completion of the fieldwork in the restoration sites, volunteers surveyed the planted Torchwood and Wild Lime for eggs and caterpillars by scanning newly emerged leaves. 
We conducted volunteer fieldwork days with a number of groups, including the Sierra Club of Miami, FIU biology students, FIU Insect Conservation Club, University of Miami (UM) Ecosystem Science and Policy undergraduates, UM Alternative Fall Break Group, Miami Dade College Environmental Science class, and Doral High School 11th and 12th graders. Before volunteers left the field site, we gave them t-shirts with a graphic that read Schaus' Swallowtail Habitat Helper to reward their hard work and to raise public awareness of the project and mission.

\section{Ex situ outreach and educational program}

Five public schools, 1 university, 1 community college, and 1 community center participated in the Schaus and Coastal Hardwood Hammock curriculum unit. The curriculum unit followed the Learning Cycle Teaching Approach to actively engage students, in which we created lessons for the student investigations modeled on the " 5 Es" (engage, explore, explain, extend, evaluate; Bybee 2002, Settlage and Southerland 2012). The lesson design highlighted several important aspects of teaching toward the Next Generation Science Standards (Achieve, Inc. 2013, Bybee 2014): (a) establishing meaningful context, (b) engaging in scientific inquiry, (c) collaborating to share/refine understanding, (d) utilizing learning tools, and (e) creating class/individual artifacts. Each participating school followed the same curriculum protocol for 3 months.

Lesson 1. The plight of the butterfly (historical lessons about Biscayne National Park). Students formed small groups and constructed a model of an island, considered ways to attract and maintain a population of Schaus' Swallowtails, and proposed possible 
solutions to ameliorate extinction after 3 kinds of disturbances (anthropogenic habitat development, application of mosquito insecticides, and tropical storms).

Lesson 2: Pin the Schaus' Swallowtail in the right habitat. After an interactive lecture presentation on imperiled butterflies of south Florida (including basic butterfly information, native plant gardening, and insect conservation), students participated in an activity titled "Place the Schaus' Swallowtail in its habitat (current range)." A randomly selected student was blindfolded and given a butterfly magnet. The remaining students brainstormed a plan to navigate the blindfolded student with the butterfly to the appropriate location on a large poster map displaying the historic and current range of the Schaus' Swallowtail (Fig. 1). Students were only allowed to communicate nonverbally, by using musical instruments (harmonica, flute, kazoo, and tambourine), until the blindfolded person placed the butterfly on the map.

Lesson 3: Habitat rehabilitation on school grounds. Students performed a butterfly survey on their school grounds using south Florida butterfly identification guides. The guides also served as a reference to let students see what host plants were appropriate for recruitment of new species and to encourage increases in local butterfly populations. Most of the species students planted in the butterfly gardens naturally occur in hardwood hammocks and pine rockland ecosystems - globally imperiled ecosystems found in south Florida (Alonso and Heinen 2011, Janzen 1988, Ross et al. 2009, Snyder et al. 1990). Several months after garden completion, students conducted another butterfly survey to compare and contrast species recruitment and abundance with their initial observations.

Lesson 4: Hardwood hammock restoration and butterfly monitoring project. FIU education students restored parts of the FIU Nature Preserve by planting native hardwood 
hammock and pine rockland plants, and, with training, participated in an on-campus butterfly monitoring project (including tagging, release, and retrieval; MonarchWatch 2014). These students (different groups each semester) learned how to estimate population size using mark-recapture techniques. They tagged Heliconius charithonia (Zebra Longwing), a common and slow-flying species of butterfly found in hardwood hammocks, in the FIU Nature Preserve for 3 semesters (Fall 2014, Spring 2015, and Summer 2015) to track butterfly movement on campus, monitor their population, and learn new skills useful for careers in education and science. Students captured Zebra Longwings with butterfly nets and placed a non-toxic label (with a specific code) on the hindwing of the butterfly. Tagged butterflies were released back into the preserve; 2 weeks later, students again caught Zebra Longwings to see how many were recaptured (Fig. 3).

\section{RESULTS}

\section{$\underline{\text { Schaus' swallowtail habitat enhancement project }}$}

This project utilized over 150 volunteers from the general public; the volunteers spent 2670 field hours (valued at $\sim \$ 60,000$, in 2015 dollars). Overall, participants planted more than 3200 plants at Elliott and Adams Keys. The main species used in restoration was Torchwood (Table 1), which participants planted in the hardwood hammock near trails at Elliott Key, connecting wild Torchwood patches to the main restoration site.

On 16 May 2015, a Schaus' Swallowtail egg was documented on 1 of the planted Torchwoods in the main restoration site at Elliott Key (Fig. 4). Students documented recruitment (larvae and eggs) of 2 species closely related to the Schaus' Swallowtail, 
Heraclides cresphontes (Giant Swallowtail) and Heraclides andraemon (Bahamian Swallowtail).

Ex situ outreach and educational program (schools and community center)

Overall, we distributed 34 plant species (31 native) to the schools and community center (Table 2). Most of the plants were used to rehabilitate the school grounds; however, students at Miami-Dade County Hialeah Campus were encouraged to plant seedlings at home and establish their own butterfly garden. Twenty-one of the plant species used were butterfly host plants (Table 3); the other 13 plant species were nectarproducing and structural plants for insects and other garden inhabitants.

When the results of the pre- and post-surveys conducted by students at 5 of the 7 survey locations were compared, we found that butterflies increased in abundance (Table 4). Students had learned a considerable amount about butterflies and they conducted the post-survey without assistance from their instructors; the instructors were present only to validate the post-survey results.

Participants tagged a total of 63 butterflies. Of these, 1 female Zebra Longwing tagged on 21 October 2014 in the FIU Nature Preserve was recaptured by a student 425 m away, near the center of campus on 16 November 2014. Four Zebra Longwings tagged on 4 June 2015 were recovered ( 3 males, 1 female) 2 weeks later (18 June 2015) near the same location in the FIU Nature Preserve. 


\section{DISCUSSION}

BNP is a sanctuary for the Schaus' Swallowtail and other imperiled organisms that depend on healthy hardwood hammocks. Because BNP maintains the largest Schaus' Swallowtail population in the Florida Keys, it is imperative to maintain the park's ecological integrity (Saunders and Hobbs 1989, USFWS 2008). As human populations continue to increase, land is continually developed, and there is pressure on the tracts surrounding protected areas. In addition, people are increasingly engulfed in cocoons provided by their enhanced mobile technology, and many have become disconnected from nature. A negative feedback pattern has been generated by these phenomena, where species may persist in protected parks but still face the threat of habitat loss and degradation near park boundaries (Pergams and Zaradic 2006, Wiersma et al. 2004).

This intensive, multi-year, service-learning project raised awareness about a federally endangered butterfly, and demonstrated memorable hands-on approaches to proactive solutions. Habitat restoration efforts led to visitation of sites and oviposition on planted Torchwood by Schaus' Swallowtails (Fig. 4), as well as oviposition by the rare Bahamian Swallowtail Butterfly. Project volunteers saw many other species at the restoration sites, including native bees, flies, resident and neotropical migrant birds, and giant land and hermit crabs, exposing them to BNP's terrestrial biodiversity. Every participating volunteer at BNP, the schools, and community center became aware of the Schaus' Swallowtail's conservation status, as well as other butterflies and their native host plants.

The schools that integrated the Schaus and Coastal Hardwood Hammock curriculum unit into their lesson plans engaged students in the garden and used field guides to identify and count various butterfly species. Students of all ages were able to conduct the 
butterfly count without the aid of teachers and other experts, and were able to identify more species during the post-survey, a prime example of "scaffolding." Scaffolding refers to students accomplishing something they, under other circumstances, would be unable to achieve, with only minimal support from a teacher (Settlage and Southerland 2012). This teacher support is temporary, and can be withdrawn as students acquire confidence, skills, and knowledge. At participating schools, students absorbed the information and used it in a multitude of ways, taking ownership and sharing with others, and working as scientists themselves.

The gardens still flourish, and both new and veteran students continue to add native plants each year while maintaining their gardens through weeding, watering, and edging. Each garden serves to enhance academic achievement, social capital, and environmental quality through active learning, applied and practical science, and self-governing responsibilities (student-driven ownership; Blair 2009, Sobel 2005). Students who value habitat preservation for wildlife in their school garden become protective of the plants they nurtured after placing them to the ground. Teachers use the garden not only for science, but also for activities in other subject areas such as mathematics, writing, and art.

Schools can raise awareness and motivate large numbers of people to think globally by acting locally. School grounds can be more ecologically valuable than the traditional grass monoculture bordered with several exotic plant species. By establishing butterfly gardens during the project, these areas became complex, multi-layered, verdant landscapes harboring diverse insect species. Teachers can use the school's surroundings as a framework upon which students can build their own learning while also increasing the biodiversity of organisms (Lieberman and Hoody 1998, Skelly and Bradley 2000). 
Projects involving schoolyard habitats can also encourage further activities by families and lifelong compassion for the environment, as well as provide guidelines for our behavior towards other people in the outdoors and our behavior towards nature (Matthews and Riley 1995, Waliczek and Zajicek 1999). By greening their school grounds and neighborhoods, teachers, students, and families can help butterflies and other insect species overcome habitat loss in areas that still harbor imperiled species.

South Florida and the Keys provide extreme examples of human habitation and development at the expense of native wildlife (Alonso and Heinen 2011, Bancroft et al. 1995, Karim and Main 2009). Education can bring about change through an increased understanding of the importance of maintaining wildlife habitat, and a sense of empowerment that individuals can do something, even on a small scale, to help promote species diversity (Miller 2005). Butterflies can be used as flagship species to educate and raise public awareness of many important environmental issues because they are charismatic and provide attractive models for conservation (Guiney and Oberhauser 2009, Leader-Williams and Dublin 2000, Walpole and Leader-Williams 2002). The establishment of butterfly gardens, such as those created for the Schaus and Coastal Hardwood Hammock curriculum unit, can provide habitat for other vulnerable species and generate an "umbrella" that can protect multiple species against negative human impacts (Guiney and Oberhauser 2009, Malone et al. 2015, Mathew and Anto 2007, Vickery 1995). 


\section{LITERATURE CITED}

Achieve, Inc. 2013. Next generation science standards (NGSS). Available online at http://www.nextgenscience.org/next-generation-science-standards. Accessed 5 September 2015.

Alonso, J., and J.T. Heinen. 2011. Miami-Dade County's environmentally endangered lands program: Local efforts for a global cause. Natural Areas Journal 3:183-189.

Armentano, T.V., D.T. Jones, M.S. Ross, and B.W. Gamble. 2002. Vegetation pattern andprocess in tree islands of the southern Everglades and adjacent areas. Pp. 225282, In F.H. Sklar and A. Van Der Valk (Eds). Tree Islands of the Everglades. Kluwer Academic Publishers, Dordrecht, The Netherlands. 541 pp.

Bancroft, G.T., A.M. Strong, and M. Carrington. 1995. Deforestation and its effects on forest-nesting birds in the Florida Keys. Conservation Biology 9:835-844.

Bender, D.J., T.A. Contreras, and L. Fahrig. 1998. Habitat loss and population decline: A meta-analysis of the patch-size effect. Ecology 79:517-533.

Berger, J.J. 1993. Ecological restoration and nonindigenous plant species: A review. Restoration Ecology 1:74-82.

Blair, D. 2009. The child in the garden: An evaluative review of the benefits of school gardening. The Journal of Environmental Education 40:15-38.

Bögeholz, S. 2006. Nature experience and its importance for environmental knowledge, values, and action: Recent German empirical contributions. Environmental Education Research 12:65-84.

Bybee, R. 2002. Learning Science and the Science of Learning: Science Educators' Essay Collection. National Science Teachers Association Press, Arlington, VA. 158 pp.

Bybee, R.W. 2014. NGSS and the next generation of science teachers. Journal of Science Teacher Education 25:211-221.

Calhoun, J.V., J.R. Slotten, and M.H. Salvato. 2002. The rise and fall of tropical blues in Florida: Cyclargus ammon and Cyclargus thomasi bethunebakeri (Lepidoptera: Lycaenidae). Holarctic Lepidoptera 7:13-20.

Chawla, L. 1998. Significant life experiences revisited: A review of research on sources of environmental sensitivity. Environmental Education Research 4:369-382. 
Dennis, R.L., J.G. Hodgson, R. Grenyer, T.G. Shreeve, and D.B. Roy. 2004. Host plants and butterfly biology. Do host-plant strategies drive butterfly status? Ecological Entomology 29:12-26.

Duda, M.D., S.J. Bissell, and K.C. Young. 1998. Wildlife and the American Mind: Public Opinion on and Attitudes toward Fish and Wildlife Management. Responsive Management, Harrisonburg, VA. 804 pp.

Egan, D., and E.A. Howell (Eds.). 2001. The Historical Ecology Handbook: A Restorationist's Guide to Reference Ecosystems. Island Press, Washington, DC. 457 pp.

Florida Invasive Species Partnership (FISP). 2017. Florida Keys Invasive Exotics Task Force. Available online at https://www.floridainvasives.org/Keys/. Accessed 5 August 2017.

Guiney, M.S., and K.S. Oberhauser. 2009. Insects as flagship conservation species. Terrestrial Arthropod Reviews 1:111-123.

Hoekstra, J.M., T.M. Boucher, T.H. Ricketts, and C. Roberts. 2005. Confronting a biome crisis: Global disparities of habitat loss and protection. Ecology Letters 8:23-29.

Horvitz, C.C., and A. Koop. 2001. Removal of nonnative vines and post-hurricane recruitment in tropical hardwood forests of Florida. Biotropica 33:268-281.

Hungerford, H., and T. Volk. 1990. Changing learner behavior through environmental education. Journal of Environmental Education 21:8-21.

Invasive.org. 2017. Florida Keys Invasive Species Task Force - List of invasive plants. Available online at https://www.invasive.org/species/list.cfm?id=82. Accessed 5 August 2017.

Jameson, A. 2002. Host plants and habitats of the Schaus Swallowtail Butterfly (Papilio aristodemus ponceanus). M.Sc. Thesis. University of Miami, Coral Gables, FL. 51 pp.

Janzen, D.H. 1988. Tropical dry forests: The most endangered major tropical ecosystem. Pp. 130-137, In E.O. Wilson and F.M. Peter (Eds.). Biodiversity. National Academies Press, Washington, DC. 521 pp.

Karim, A., and M.B. Main. 2009. Habitat fragmentation and conservation strategies for a rare forest habitat in the Florida Keys archipelago. Urban Ecosystems 12:359-370. 
Leader-Williams N., and H. Dublin. 2000. Charismatic megafauna as "flagship species". Pp. 53-81, In A. Entwistle and N. Dunstone (Eds.). Priorities for the Conservation of Mammalian Diversity: Has the Panda had its Day? Cambridge University Press, Cambridge, UK. 457 pp.

Lieberman, G.A., and L.L. Hoody. 1998. Closing the achievement gap: Using the environment as an integrating context for learning. Research report. State Environment and Education Roundtable, San Diego, CA. 21 pp.

Loftus, W., and J. Kushlan. 1984. Population fluctuations of the Schaus Swallowtail (Lepidoptera: Papilionidae) on the islands of Biscayne Bay, Florida, with comments on the Bahaman Swallowtail. Florida Entomologist 67:277-287.

Louv, R. 2008. Last Child in the Woods: Saving our Children from Nature-deficit Disorder. Algonquin Books, New York, NY. 323 pp.

Malone, K.C., W. Wilber, G. Hansen, J.C. Daniels, C. Larsen, and E. Momol. 2015. Community butterfly-scaping: How to move beyond butterfly gardening to create a largescale butterfly habitat. University of Florida IFAS Extension. Available online at http://edis.ifas.ufl.edu/ep420. Accessed 9 September 2015.

Mathew, G., and M. Anto. 2007. In situ conservation of butterflies through establishment of butterfly gardens: A case study at Peechi, Kerala, India. Current Science 93:337347.

Matthews, B.E., and C.K. Riley. 1995. Teaching and evaluating outdoor-ethics education programs. Descriptive report. National Wildlife Federation, ERIC Document Reproduction Service No. ED 401 097, Vienna, VA. 118 pp.

Miller, J.R. 2005. Biodiversity conservation and the extinction of experience. Trends in Ecology and Evolution 20:430-434.

Minno, M.C., and T.C. Emmel. 1993. Butterflies of the Florida Keys. Scientific Publishers, Gainesville, FL. 168 pp.

MonarchWatch. 2014. MonarchWatch: Migration and tagging. Available online at http:// monarchwatch.org/tagmig/tag.htm. Accessed 16 March 2014.

Myers, R.L., and J.J. Ewel (Eds.). 1990. Ecosystems of Florida. University of Central Florida Press, Orlando, FL. 765 pp.

National Park Service (NPS). 2015. National Park Service visitor-use statistics. Available online at https://irma.nps.gov/Stats/Reports/Park/BISC. Accessed 9 September 2015. 
Oliver, T., D.B. Roy, J.K. Hill, T. Brereton, and C.D. Thomas. 2010. Heterogeneous landscapes promote population stability. Ecology Letters 13:473-484.

Pergams, O.R., and P.A. Zaradic. 2006. Is love of nature in the US becoming love of electronic media? 16-year downtrend in national park visits explained by watching movies, playing video games, internet use, and oil prices. Journal of Environmental Management 80:387-393.

Pergams, O.R., and P.A. Zaradic. 2008. Evidence for a fundamental and pervasive shift away from nature-based recreation. Proceedings of the National Academy of Sciences 105:2295-2300.

Pyle, R.M. 1978. The extinction of experience. Horticulture 56:64-67.

Ray, G.J., F. Dallmeier, and J.A. Comiskey. 1998. The structure of two subtropical dry forest communities on the island of St. John, US Virgin Islands. Pp. 367-384, In F. Dallmeier and J.A. Comiskey (Eds.). Forest Biodiversity in North, Central, and South America, and the Caribbean: Research and Monitoring, Man and the Biosphere series. Vol. 21. UNESCO and the Parthenon Publishing Group, Camforth, Lancashire, UK. $768 \mathrm{pp}$.

Ricketts, T. 2001. The matrix matters: Effective isolation in fragmented landscapes. The American Naturalist 158:87-99.

Ricketts, T.H., J. Regetz, I. Steffan-Dewenter, S.A. Cunningham, C. Kremen, A. Bogdanski, B. Gemmill-Herren, S.S. Greenleaf, A.M. Klein, M.M. Mayfield, L.A. Morandin, A. Ochieng, and B.F. Viana. 2008. Landscape effects on crop-pollination services: Are there general patterns? Ecology Letters 11:499-515.

Roe, D., N. Leader-Williams, and D.B. Dalal-Clayton. 1997. Take only photographs, leave only footprints: The environmental impacts of wildlife tourism (No. 10). The International Institute for Environment and Development, London, UK. 86 pp.

Rosa, E., R. York, and T. Dietz. 2004. Tracking the anthropogenic drivers of ecological impacts. AMBIO: A Journal of the Human Environment 33:509-512.

Ross, M.S., J.J. O’Brien, and L.J. Flynn. 1992. Ecological site classification of Florida Keys terrestrial habitats. Biotropica 24:488-502.

Ross, M.S., M. Carrington, L.J. Flynn, and P.L. Ruiz. 2001. Forest succession in tropical hardwood hammocks of the Florida Keys: Effects of direct mortality from Hurricane Andrew. Biotropica 33:23-33. 
Ross, M.S., J.J. O’Brien, R.G. Ford, K. Zhang, and A. Morkill. 2009. Disturbance and the rising tide: The challenge of biodiversity management on low-island ecosystems. Frontiers in Ecology and the Environment 7:471-478.

Rutkowski, F. 1971. Observations on Papilio aristodemus ponceanus (Papilionidae). Journal of Lepidopterist Society 25:126-136.

Saunders, D., and R. Hobbs. 1989. Corridors for conservation. New Scientist 121:63-68.

Settlage, J., and S.A. Southerland. 2012. Teaching Science to Every Child: Using Culture as a Starting Point. Taylor and Francis, New York, NY. 383 pp.

Skelly, S.M., and J.C. Bradley. 2000. The importance of school gardens as perceived by Florida elementary school teachers. Horticultural Technology 10:229-231.

Smith, D.S., L.D. Miller, J.Y. Miller, and R. Lewington. 1994. The Butterflies of the West Indies and South Florida. Oxford University Press, New York, NY. 264 pp.

Snyder J.R., A. Herndon, and W.B.J. Robertson. 1990. South Florida rockland. Pp. 230277, In R.L. Myers and J.J. Ewel (Eds.). Ecosystems of Florida. University Press of Florida, Orlando, FL. 765 pp.

Sobel, D. 2005. Place-based education: Connecting Classrooms and Communities. The Orion Society, Great Barrington, MA. 116 pp.

Stevens, T.H., T.A. More, and M. Markowski-Lindsey. 2014. Declining national park visitation. Journal of Leisure Research 46:153-164.

Strong, A.M., and G.T. Bancroft. 1994. Patterns of deforestation and fragmentation of mangrove and deciduous seasonal forests in the upper Florida Keys. Bulletin of Marine Science 54:795-804.

Taki, H., and P.G. Kevan. 2007. Does habitat loss affect the communities of plants and insects equally in plant-pollinator interactions? Preliminary findings. Biodiversity and Conservation 16:3147-3161.

US Fish and Wildlife Service (USFWS). 2008. Schaus' Swallowtail Butterfly (Heraclides aristodemus ponceanus) 5-year review: Summary and evaluation. Available online at http://ecos.fws.gov/docs/five_year_review/doc1983.pdf. Accessed 23 Oct 2013.

USFWS. 2015. Environmental conservation online system (Schaus' Swallowtail Butterfly). Available online at https://ecos.fws.gov/ecp0/profile/speciesProfile?spcode=I016. Accessed 8 September 2015. 
Waliczek, T.M., and J.M. Zajicek. 1999. School gardening: Improving environmental attitudes of children through hands-on learning. Journal of Environmental Horticulture 17:180-184.

Walpole, M.J., and N. Leader-Williams. 2002. Tourism and flagship species in conservation. Biodiversity and Conservation 11:543-547.

Wells, N.M., and K.S. Lekies. 2006. Nature and the life course: Pathways from childhood nature experiences to adult environmentalism. Children, Youth, and Environments $16: 1-24$.

Whelan, K.R. 2011. Schaus' Swallowtail Butterfly (Heraclides aristodemus ponceanus) habitat enhancement in Biscayne National Park. Project report. Funded by FWS South Florida Coastal Program. National Park Service, Palmetto Bay, FL. 9 pp.

Whelan, K.R.T., and A. Atkinson. 2015. Schaus' Swallowtail Butterfly (Heraclides aristodemus ponceanus) habitat enhancement in Biscayne National Park. South Florida Coastal Program project progress report. NPS/SFCN. National Park Service, Palmetto Bay, FL. 24 pp.

Whelan, K.R.T., P.L. Ruiz, R.B. Shamblin, P.A. Houle, M.S. Ross, A.J. Atkinson, J.M. Patterson, and J. Alonso. 2013. Biscayne National Park vegetation map project. Natural resource technical report. NPS/SFCN/NRTR - 2013/774. National Park Service, Fort Collins, CO. 352 pp.

Wiersma, Y.F., T.D. Nudds, and D.H. Rivard. 2004. Models to distinguish effects of landscape patterns and human population pressures associated with species loss in Canadian national parks. Landscape Ecology 19:773-786.

Vickery, M.L. 1995. Gardens: The neglected habitat. Pp. 123-134, In A.S. Pullin (Ed.). Ecology and Conservation of Butterflies. Springer, Dordrecht, The Netherlands. 363 pp.

Zaradic, P.A., and O.R. Pergams. 2007. Videophilia: Implications for childhood development and conservation. Journal of Developmental Processes 2:130-144. 
TABLES

Table 1.1: Number of plants including Torchwood, Wild Lime, and nectar plants planted at Elliott and Adams Keys. Numbers are shown per restoration area. Column on right shows total number of plants planted per restoration area.

\begin{tabular}{|c|l|c|c|c|c|c|}
\hline \multirow{2}{*}{ Island } & \multicolumn{1}{|c|}{ Restoration Area } & ha & $\begin{array}{c}\text { Torchwood } \\
\text { Planted }\end{array}$ & $\begin{array}{c}\text { Wild Lime } \\
\text { Planted }\end{array}$ & $\begin{array}{c}\text { Nectar Plants } \\
\text { Planted }\end{array}$ & $\begin{array}{c}\text { Total Plants } \\
\text { Planted to Date }\end{array}$ \\
\hline \multirow{5}{*}{ Elliott Key } & Elliott Main & 0.40 & 598 & 116 & 60 & 774 \\
\cline { 2 - 7 } & Elliott Breezeway & 0.18 & 433 & 102 & 2 & 537 \\
\cline { 2 - 7 } & Elliott Spite Highway North & N/A & 824 & - & - & 824 \\
\cline { 2 - 7 } & Elliott East & N/A & 58 & - & - & 58 \\
\cline { 2 - 7 } & Elliott West & N/A & 151 & - & - & 151 \\
\hline \multirow{2}{*}{ Adams Key } & Adams Main & 0.17 & 414 & 104 & 15 & 533 \\
\cline { 2 - 7 } & Adams Breezeway & 0.10 & 275 & 64 & 17 & 356 \\
\hline \multicolumn{1}{|l|}{ Totals } & $\mathbf{2 . 1}$ & $\mathbf{2 , 7 5 3}$ & $\mathbf{3 8 6}$ & $\mathbf{9 4}$ & $\mathbf{3 , 2 3 3}$ \\
\hline
\end{tabular}


Table 1.2: Plants planted in the butterfly gardens and FIU Nature Preserve. Note: MDC students were given plants to take home. Asterisks $(*)$ denote exotic plants. See text (Field-site Description) for site abbreviations.

\begin{tabular}{|lrrrrrrrrrr|}
\hline & \multicolumn{7}{c}{ Site } & & & \\
Plant species & MDC & FIU & OHM & AB & CTE & GEL & NHE & WPE & Total \\
\hline Alvaradoa amorphoides & 6 & 5 & 3 & 0 & 4 & 5 & 0 & 0 & 23 \\
Amyris elemifera & 0 & 4 & 1 & 0 & 2 & 0 & 0 & 0 & 7 \\
Angadenia berteroi & 14 & 7 & 6 & 3 & 8 & 6 & 0 & 4 & 48 \\
Aristolochia gigantea* & 0 & 0 & 0 & 0 & 2 & 0 & 2 & 2 & 6 \\
Asclepias curassavica* & 0 & 0 & 3 & 0 & 4 & 15 & 4 & 1 & 27 \\
Asclepias incarnata & 10 & 0 & 0 & 0 & 0 & 0 & 0 & 2 & 12 \\
Asclepias tuberosa & 12 & 10 & 0 & 0 & 1 & 3 & 0 & 0 & 26 \\
Bourreria succulenta & 4 & 0 & 0 & 1 & 0 & 0 & 2 & 0 & 7 \\
Byrsonima lucida & 6 & 5 & 3 & 3 & 3 & 0 & 0 & 3 & 23 \\
Cardiospermum corindum & 2 & 0 & 0 & 0 & 0 & 0 & 0 & 0 & 2 \\
Chamaecrista fasciculata & 0 & 0 & 0 & 0 & 8 & 0 & 0 & 12 & 20 \\
Citharexylum spinosum & 0 & 0 & 0 & 0 & 0 & 4 & 0 & 0 & 4 \\
Coccothrinax argentata & 0 & 0 & 0 & 0 & 2 & 0 & 0 & 0 & 2 \\
Colubrina elliptica & 0 & 0 & 0 & 0 & 0 & 0 & 3 & 3 & 6 \\
Cordia sebestena & 3 & 0 & 0 & 0 & 3 & 0 & 4 & 0 & 10 \\
Croton linearis & 0 & 4 & 3 & 0 & 2 & 9 & 0 & 4 & 22 \\
Cynophalla flexuosa & 2 & 6 & 0 & 3 & 5 & 4 & 0 & 1 & 21 \\
Ficus aurea & 0 & 0 & 0 & 0 & 2 & 1 & 0 & 0 & 3 \\
Flaveria linearis & 0 & 2 & 0 & 2 & 8 & 4 & 0 & 6 & 22 \\
Guaiacum sanctum & 5 & 0 & 0 & 2 & 3 & 4 & 0 & 3 & 17 \\
Guapira discolor & 8 & 2 & 0 & 0 & 2 & 0 & 0 & 0 & 12 \\
Jacquinia keyensis & 0 & 0 & 0 & 16 & 0 & 0 & 0 & 0 & 16 \\
Lantana involucrata & 10 & 8 & 0 & 4 & 4 & 0 & 0 & 6 & 32 \\
Passiflora suberosa & 14 & 3 & 3 & 10 & 20 & 8 & 5 & 20 & 83 \\
Physalis walteri & 3 & 6 & 0 & 2 & 6 & 5 & 0 & 4 & 26 \\
Pithecellobium unguis-cati & 4 & 1 & 1 & 1 & 3 & 2 & 0 & 0 & 12 \\
Plumbago zeylanica & 1 & 0 & 0 & 0 & 3 & 1 & 2 & 0 & 7 \\
Psychotria nervosa & 4 & 3 & 0 & 0 & 4 & 0 & 0 & 0 & 11 \\
Ruta graveolens* & 0 & 0 & 0 & 0 & 3 & 3 & 2 & 0 & 8 \\
Senna mexicana & 3 & 8 & 4 & 5 & 2 & 4 & 10 & 4 & 40 \\
Solidago sempervirens & 9 & 0 & 0 & 0 & 0 & 0 & 0 & 4 & 13 \\
Varronia globosa & 2 & 0 & 0 & 0 & 0 & 2 & 0 & 0 & 4 \\
Zamia integrifolia & 0 & 0 & 10 & 6 & 4 & 28 & 4 & 6 & 58 \\
Zanthoxylum fagara & 10 & 4 & 0 & 3 & 3 & 2 & 2 & 2 & 26 \\
Total & 132 & 78 & 37 & 61 & 111 & 110 & 40 & 87 & 656 \\
\hline & & & & & & & & & \\
\hline
\end{tabular}


Table 1.3: Host plants planted in the gardens and Lepidoptera (butterflies, moths, and skippers) attracted to them. Asterisks denote exotic plants.

\begin{tabular}{|c|c|}
\hline Host plants & Butterfly, moth, and skipper species that depend on each host plant \\
\hline Alvaradoa amorphoides (Mexican Alvaradoa) & Pyrisitia dina (Dina Yellow) \\
\hline Amyris elemifera (Sea Torchwood) & $\begin{array}{l}\text { Heraclides aristodemus ponceanus (Schaus Swallowtail), Heraclides andraemon (Bahamian } \\
\text { Swallowtail), Heraclides cresphontes (Giant Swallowtail) }\end{array}$ \\
\hline Angadenia berteroi (Pineland Golden Trumpet) & Syntomeida epilais (Polka-Dot Wasp Moth) \\
\hline Aristolochia gigantea' (Brazilian Dutchman's Pipe) & Battus polydamas (Polydamas Swallowtail) \\
\hline Asclepias species (Milkweed) & Danaus plexippus (Monarch), Danaus gilippus (Queen), Danaus eresimus (Soldier) \\
\hline Byrsonima lucida (Locustberry) & Ephyriades brunnea (Florida Duskywing) \\
\hline Capparis flexuosa (Limber Caper) & Glutophrissa drusilla neumoegenii (Florida White), Ascia monuste (Great Southern White) \\
\hline Cardiospermum corindum (Balloonvine) & Chlorostrymon simaethis (Silver-B anded Hairstreak) \\
\hline Chamaecrista fasciculata (Partridge Pea) & $\begin{array}{l}\text { Hemiargus ceraunus (Ceraunus Blue), Phoebis sennae (Cloudless Sulphur), Eurema nicippe } \\
\text { (Sleepy Orange), Pyrisitia lisa (Little Yellow) }\end{array}$ \\
\hline Citharexylum spinosum (Fiddlewood) & Epicorsia oedipodalis (Fiddlewood Lea froller) \\
\hline Coccothrinax argentata (Silver Palm) & Asbolis capucinus (Monk Skipper) \\
\hline Croton linearis (Pineland Croton) & Anaea troglodyta floridalis (Flor ida Leafwing), Strymon acis bartrami (B artram's Scrub-Hairstreak) \\
\hline Ficus aurea (Strangler Fig) & Marpesia petreus (Ruddy Daggerwing) \\
\hline Guaiacum sanctum (Lignum-Vitae) & Kricogonia lyside (Lyside Sulphur) \\
\hline Passiflora suberosa (Corkystem Passionflower) & Heliconius charithonia (Zebra Longwing), Dryas iulia (Julia), Agraulis vanillae (Gulf Fritillary) \\
\hline Pithecellobium unguis-cati (Catclaw B lack bead) & Leptotes cassius (Cassius Blue), Phoebis agarithe (Large Orange Sulphur) \\
\hline Plumbago zeylanica (Doctorbush) & Leptotes cassius (Cassius Blue) \\
\hline Ruta graveolens' (Rue) & Heraclides cresphontes (Giant Swallowtail), Papilio polyxenes (Black Swallowtail) \\
\hline Senna mexicana (Chapman's Senna) & Phoebis philea (Orange-Barred Sulphur), Phoebis sennae (Cloudless Sulphur) \\
\hline Zamia integrifolia (Coontie) & Eumaeus atala (Atala) \\
\hline Zanthoxylum fagara (Wild Lime) & $\begin{array}{l}\text { Heraclides aristodemus ponceamus (Schaus Swallowtail), Heraclides andraemon (Bahamian } \\
\text { Swallowtail), Heraclides cresphontes (Giant Swallowtail) }\end{array}$ \\
\hline
\end{tabular}


Table 1.4: Before garden construction, butterfly-species counts (pre-survey) were conducted (numbers indicated in parentheses) at each site. Several months later, a postsurvey was conducted in the same area at each site. Asterisks $(*)$ denote an increase in butterflies from the pre-survey. Note: Students at MDCHC did not construct a butterfly garden.

\begin{tabular}{|c|c|c|c|c|c|c|c|c|}
\hline \multirow[b]{2}{*}{ Butterfly species } & \multicolumn{7}{|c|}{ Site } & \multirow[b]{2}{*}{ Total } \\
\hline & $\mathrm{MDCHC}$ & OHMCC & AB K-8 & CTE & GEL K-8 & NHE & WPE & \\
\hline Agraulis vanillae & $(0) 0$ & $(0) 1$ & $(5) 0$ & $(0) 2$ & (1) 5 & $(0) 3$ & (2)3 & $(8) 14 *$ \\
\hline Anartia jatrophae & (3) 4 & (5) 2 & (4) 0 & (6) 3 & $(5) 5$ & (2) 2 & (4) 3 & (29) 19 \\
\hline Asbolis capucinus & $(0) 0$ & $(0) 0$ & (0)4 & (0)0 & (3) 2 & $(0) 1$ & (0)1 1 & (3) $8 *$ \\
\hline Ascia monuste & $(0) 0$ & (0) 1 & $(0) 0$ & (0)0 & $(0) 0$ & (0)3 & (0) 1 & $(0) 5^{*}$ \\
\hline Battus polydamas & $(0) 0$ & $(0) 0$ & $(0) 0$ & (0)1 & $(0) 0$ & $(0) 0$ & (1) 3 & (1) $4 *$ \\
\hline Danaus gilippus & $(0) 0$ & $(0) 0$ & $(0) 0$ & $(0) 0$ & (2) 2 & $(0) 0$ & (2) 1 & (4) 3 \\
\hline Danaus plexippus & $(0) 0$ & $(0) 3$ & (3) 0 & (5) 2 & (3) 12 & (1) 5 & (4) 5 & $(16) 27 *$ \\
\hline Dryas iulia & $(0) 0$ & (0) 2 & (1) 0 & $(0) 0$ & (0) 1 & $(0) 0$ & (1) 3 & (4) $6^{*}$ \\
\hline Electrostrymon angelia & $(0) 0$ & (1) 1 & $(0) 0$ & $(0) 0$ & (0) 1 & $(0) 0$ & $(0) 0$ & (1) $2 *$ \\
\hline Eumaeus atala & $(0) 0$ & $(0) 0$ & (2) 3 & $(0) 0$ & $(0) 0$ & $(0) 0$ & $(0) 0$ & $(2) 3 *$ \\
\hline Eurema daira & $(0) 2$ & (5) 3 & $(0) 2$ & $(8) 3$ & $(0) 0$ & (4) 2 & $(0) 0$ & $17(12)$ \\
\hline Heliconius charithonia & $(0) 0$ & (0)0 & (4) 6 & $(0) 0$ & (0) 1 & $(0) 0$ & (3) 5 & $(7) 12 *$ \\
\hline Hemiargus ceraunus & $(0) 0$ & $(0) 0$ & (1) 1 & (0)2 & (5) 2 & $(0) 0$ & (2) 1 & $(8) 6$ \\
\hline Hylephila phyleus & $(0) 0$ & $(0) 1$ & $(0) 3$ & $(0) 0$ & $(0) 0$ & $(0) 0$ & $(0) 0$ & $(0) 4 *$ \\
\hline Junonia coenia & $(0) 0$ & (0) 1 & (1) 1 & $(0) 0$ & (2) 1 & $(0) 0$ & (2) 1 & (5) 4 \\
\hline Leptotes cassius & (3) 1 & (0) 2 & (1) 0 & (3) 3 & (6) 8 & (1)3 & (0)2 & (14) $19 *$ \\
\hline Marpesia petreus & $(0) 0$ & $(0) 0$ & (0) 1 & (0)0 & $(0) 0$ & $(0) 0$ & (1) 1 & $(1) 2^{*}$ \\
\hline Nathalis iole & $(0) 0$ & (3) 1 & (2) 0 & (1) 4 & (0)3 & (0) 1 & $(0) 0$ & $(6) 9 *$ \\
\hline Papilio cresphontes & $(0) 0$ & $(0) 0$ & (1) 0 & (0) 1 & $(0) 0$ & (1) 1 & $(0) 0$ & (2) 2 \\
\hline Papilio polyxenes & $(0) 0$ & $(0) 0$ & $(0) 0$ & $(0) 0$ & $(0) 1$ & $(0) 0$ & $(0) 0$ & $(0) 1 *$ \\
\hline Phoebis agarithe & (2) 1 & (4) 2 & (3) 2 & $(0) 0$ & $(10) 9$ & (1) 0 & $(0) 0$ & (20)14 \\
\hline Phoebis philea & (0)0 & (0)0 & (0) 1 & (0) 1 & $(2) 1$ & (0)0 & (0)0 & $(2) 3 *$ \\
\hline Phoebis sennae & $(0) 0$ & $(0) 0$ & $(0) 0$ & $(0) 2$ & $(0) 1$ & $(0) 1$ & $(0) 0$ & $(0) 4 *$ \\
\hline Phyciodes phaon & $(0) 0$ & (1) 1 & (1) 0 & $(0) 0$ & (1) 1 & $(0) 0$ & (2) 1 & (5) 3 \\
\hline Polites vibex & $(0) 0$ & $(0) 0$ & (0)1 & $(0) 0$ & $(0) 0$ & (1) 0 & (0) 1 & (1) $2 *$ \\
\hline Pyrgus oileus & $(0) 0$ & (1) 1 & (0)0 & $(0) 0$ & $(0) 0$ & $(0) 0$ & (1) 1 & $(2) 2$ \\
\hline Urbanus proteus & $(0) 0$ & (1) 0 & $(0) 0$ & $(0) 0$ & $(0) 0$ & $(0) 1$ & $(0) 1$ & (1) $2 *$ \\
\hline Total & (8) 8 & $(21) 22 *$ & (29)25 & (23) $24 *$ & $(40) 56^{*}$ & (11) $23 *$ & $(27) 34 *$ & (159)192* \\
\hline
\end{tabular}




\section{FIGURES}

Figure 1.1: Current range of the Schaus' Swallowtail Butterfly in south Florida. Map generated by Helena Giannini.

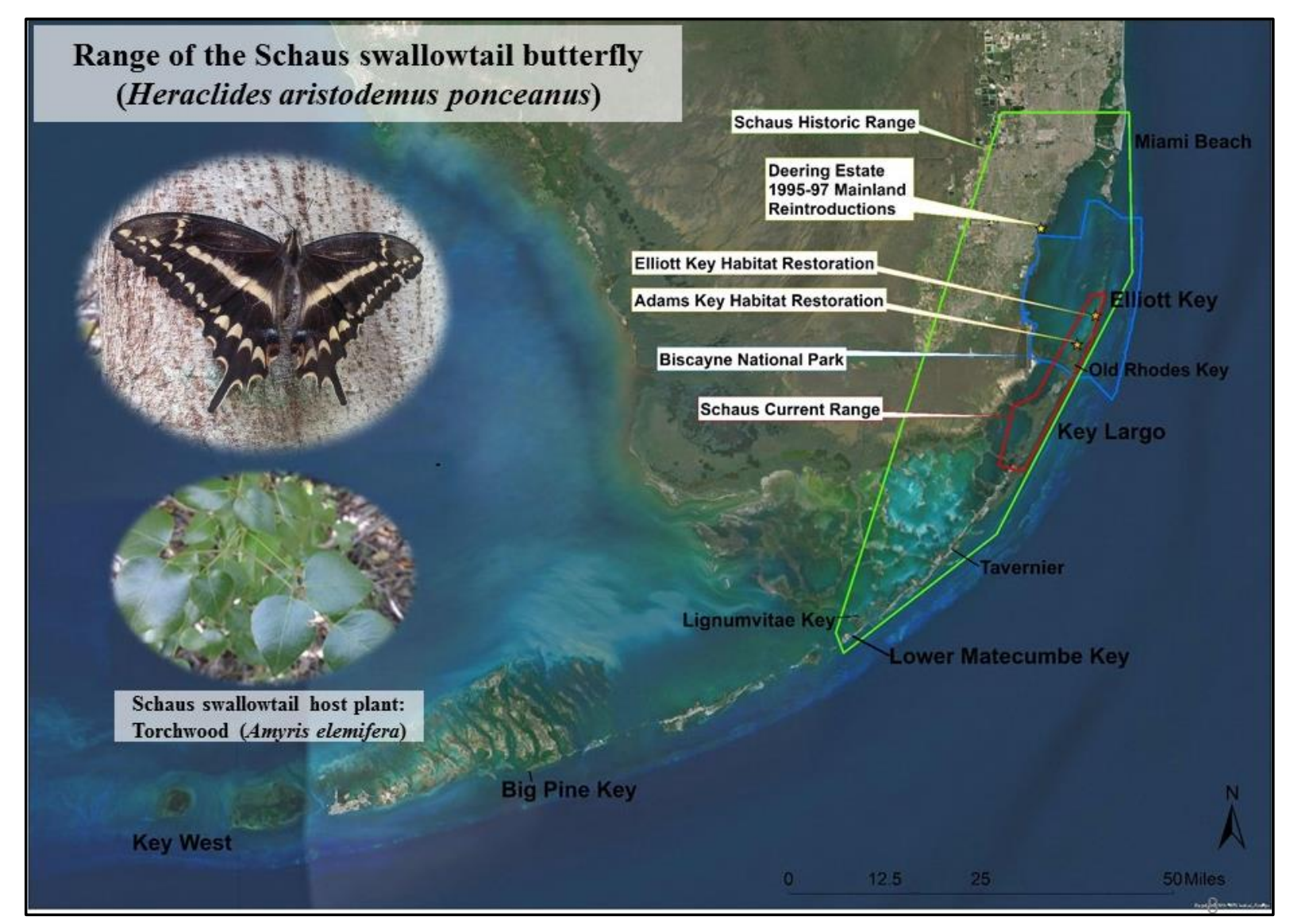


Figure 1.2: Study-site locations in Miami-Dade County (Biscayne National Park is outlined on the bottom right in the map). See text (Field-site Description) for site abbreviations.

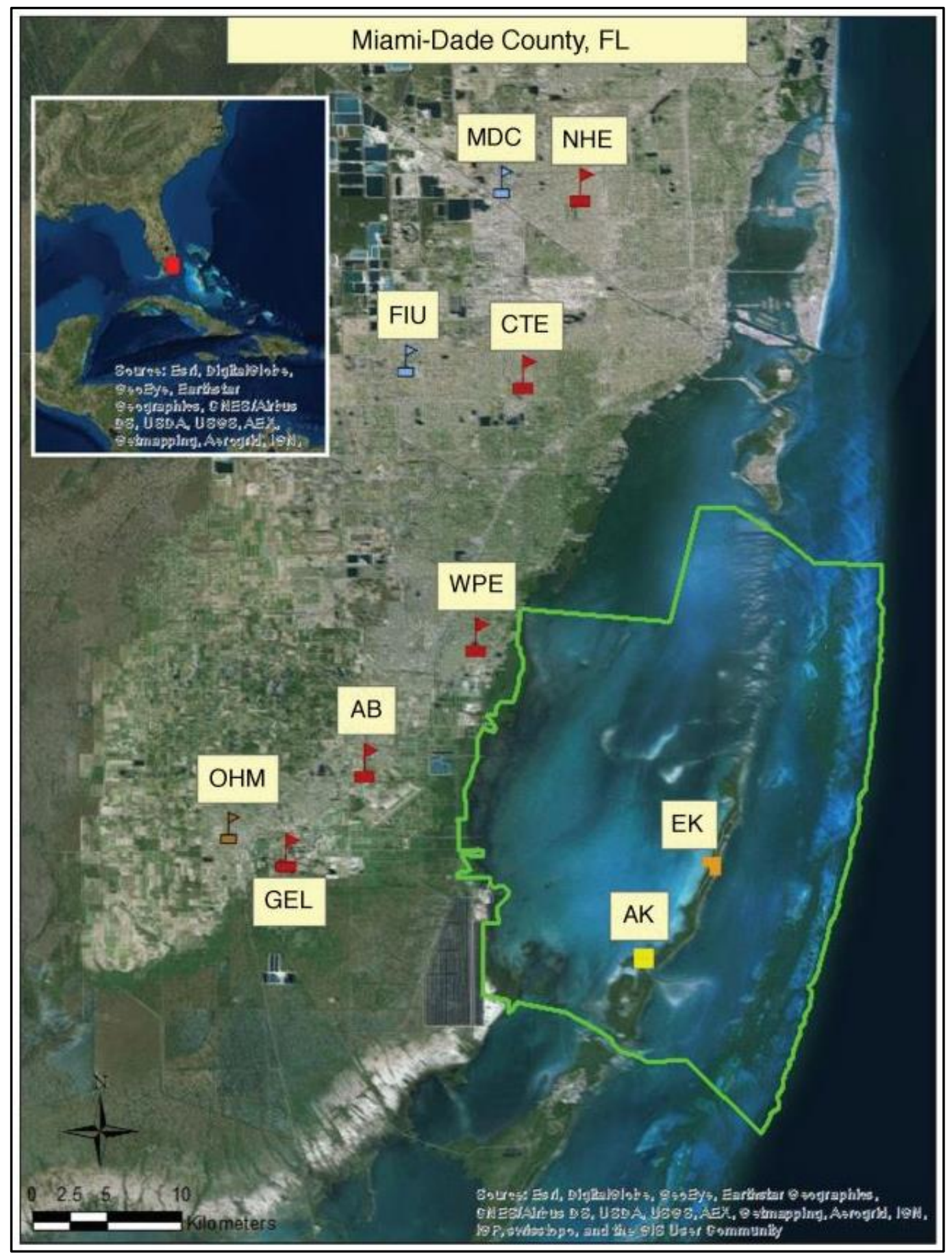


Figure 1.3: Tagged Zebra Longwing ready for release at the Florida International University nature preserve.

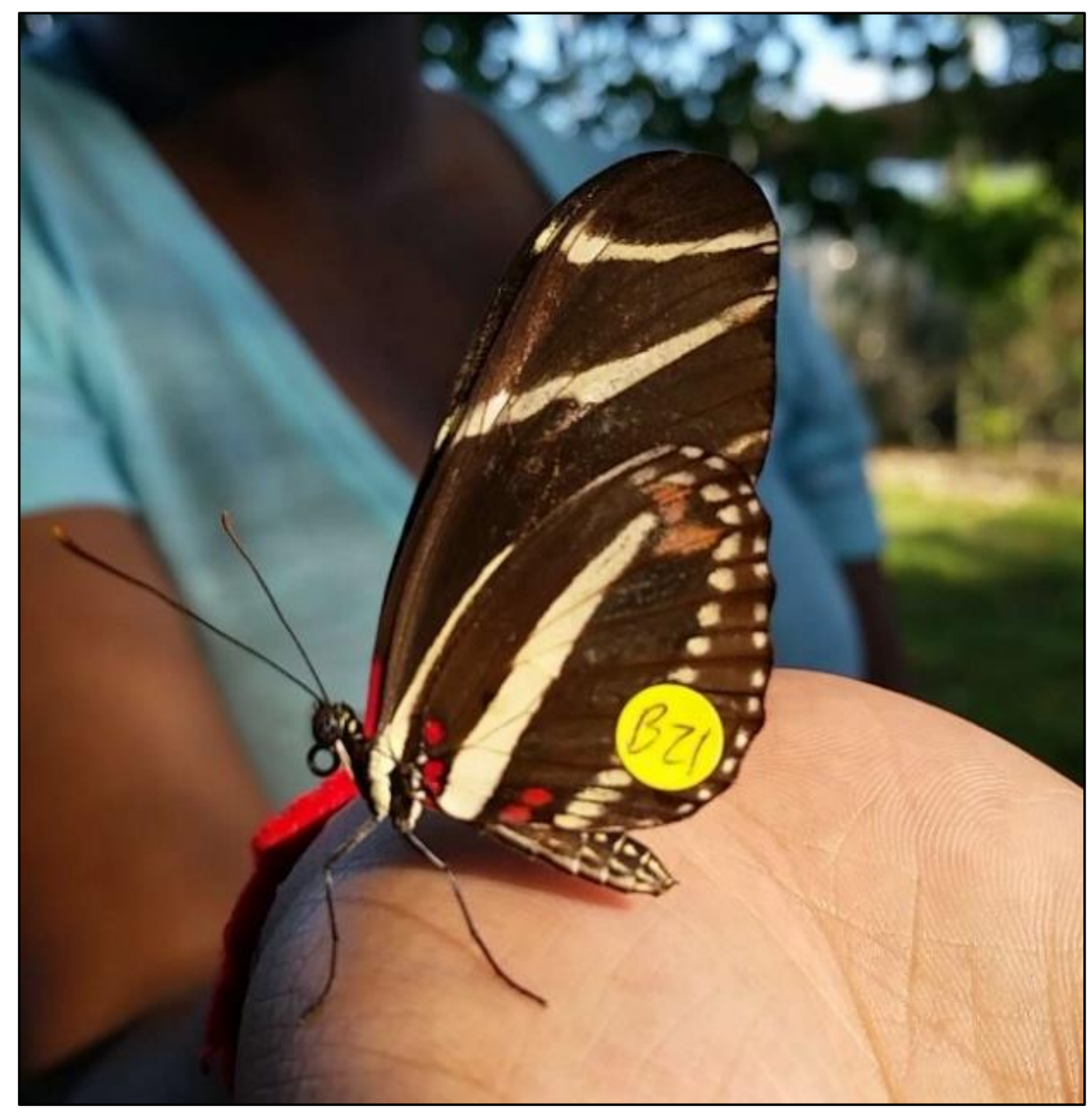


Figure 1.4: Schaus' Swallowtail egg on Torchwood in the main restoration site at Elliott Key in Biscayne National Park (left); Giant Swallowtail caterpillar on Wild Lime in the main restoration site at Adams Key (right).

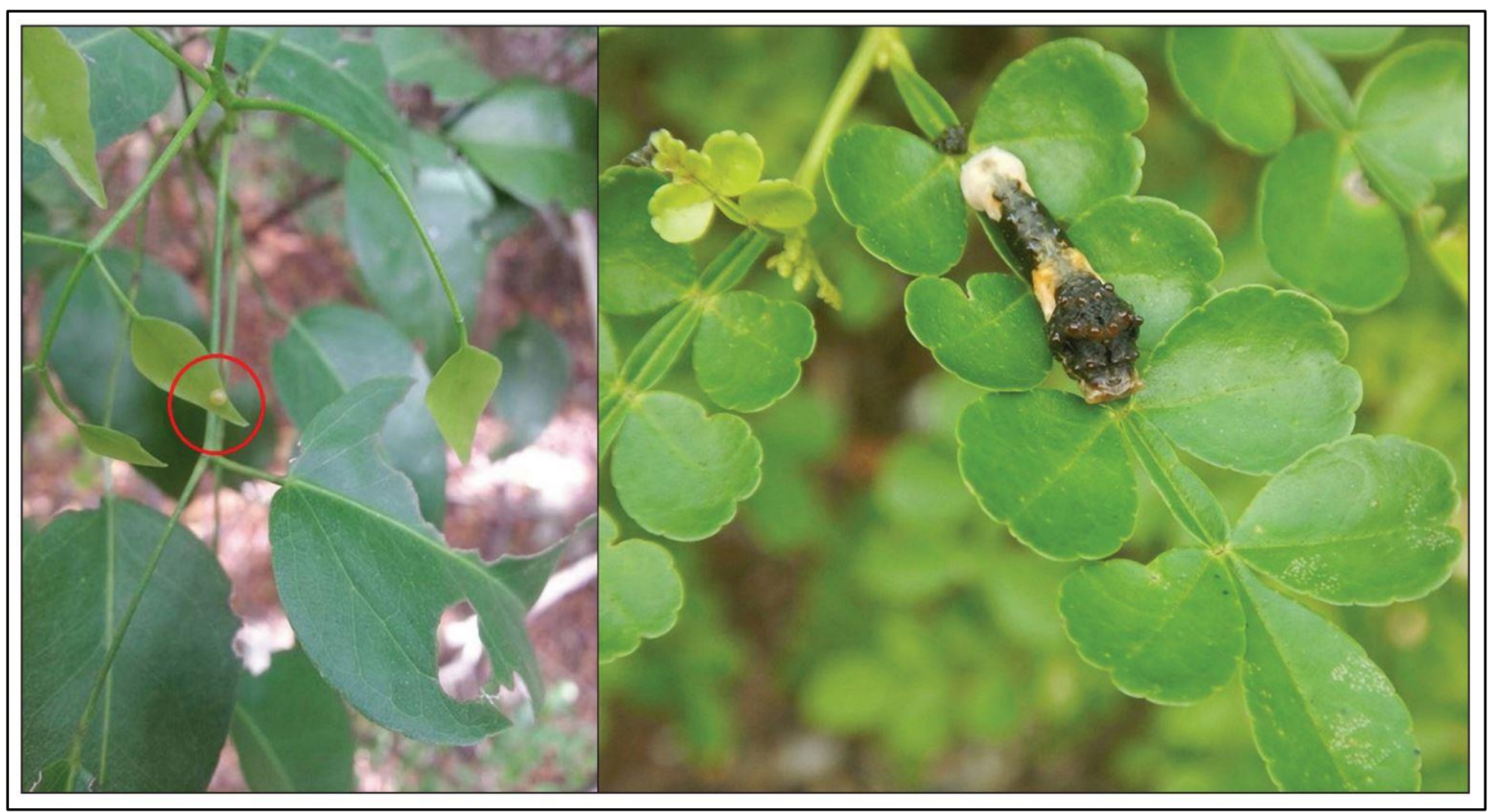




\section{APPENDICES}

Appendix 1.1: Latin names, taxonomic authority, and common names of butterfly, moth, and skipper species mentioned in this paper.

\begin{tabular}{|c|c|}
\hline Scientific name & Common name \\
\hline Abaeis nicippe (Cramer) & Sleepy Orange \\
\hline Agraulis vanillae nigrior Michener & Gulf Fritillary \\
\hline Anaea troglodyta floridalis F. Johnson \& W.P. Comstock & Florida Leafwing \\
\hline Anartia jatrophae guantanamo Munroe & White Peacock \\
\hline Asbolis capucinus (Lucas) & Monk Skipper \\
\hline Ascia monuste (L.) & Great Southern White \\
\hline Battus polydamas lucayus (Rothschild and Jordan) & Polydama Swallowtail \\
\hline Chlorostrymon simaethis (Drury) & Silver-banded Hairstreak \\
\hline Danaus eresimus tethys W. Forbes & Soldier \\
\hline Danaus gilippus berenice (Cramer) & Queen \\
\hline Danaus plexippus (L.) & Monarch \\
\hline Dryas iulia largo Clench & Julia \\
\hline Electrostrymon angelia (Hewitson) & Fulvous Hairstreak \\
\hline Ephyriades brunnea floridensis E. Bell \& W. Comstock & Florida Duskywing \\
\hline Epicorsia oedipodalis (Guenée) & Fiddlewood Leafroller \\
\hline Eumaeus atala Poey & Atala \\
\hline Eurema daira (Godart) & Barred Yellow \\
\hline Glutophrissa drusilla neumoegenii (Skinner) & Florida White \\
\hline Heliconius charithonia tuckeri W. Comstock \& F. Brown & Zebra Longwing \\
\hline Hemiargus ceraunus antibubastus Hübner & Ceraunus Blue \\
\hline Hylephila phyleus (Drury) & Fiery Skipper \\
\hline Junonia coenia Hübner & Common Buckeye \\
\hline Kricogonia lyside (Godart) & Lyside Sulphur \\
\hline Leptotes cassius theonus (Lucas) & Cassius Blue \\
\hline Marpesia petreus (Cramer) & Ruddy Daggerwing \\
\hline Nathalis iole Boisduval & Dainty Sulphur \\
\hline Papilio (Heraclides) andraemon (Hübner) & Bahamian Swallowtail \\
\hline Papilio (Heraclides) aristodemus ponceanus (Schaus) & Schaus Swallowtail \\
\hline Papilio (Heraclides) cresphontes Cramer & Giant Swallowtail \\
\hline Papilio polyxenes asterius (Stoll) & Black Swallowtail \\
\hline Phoebis agarithe maxima (Neumoegen) & Large Orange Sulphur \\
\hline Phoebis philea (L.) & Orange-barred Sulphur \\
\hline Phoebis sennae (L.) & Cloudless Sulphur \\
\hline Phyciodes phaon (W.H. Edwards) & Phaon Crescent \\
\hline Polites vibex (Geyer) & Whirlabout \\
\hline Pyrgus oileus (Linnaeus) & Tropical Checkered-Skipper \\
\hline Pyrisitia dina helios (D. Bates) & Dina Yellow \\
\hline Pyrisitia lisa (Boisduval \& Le Conte) & Little Yellow \\
\hline Syntomeida epilais (Walker) & Polka-Dot Wasp Moth \\
\hline Strymon acis bartrami (W. Comstock and Huntington) & Bartram's Scrub-Hairstreak \\
\hline Urbanus proteus (L.) & Long-tailed Skipper \\
\hline
\end{tabular}


Appendix 1.2: Latin names, taxonomic authority, and common names of plants

mentioned in this paper.

\begin{tabular}{|c|c|}
\hline Scientific name & Common name \\
\hline Alvaradoa amorphoides Liebm. & Mexican Alvaradoa \\
\hline Amyris elemifera $\mathrm{L}$. & Sea Torchwood \\
\hline Angadenia berteroi (A.DC.) Miers & Pineland Golden Trumpet \\
\hline Aristolochia gigantea Mart. \& Zucc. & Brazilian Dutchman's Pipe \\
\hline Asclepias curassavica $\mathrm{L}$. & Tropical Milkweed \\
\hline Asclepias incarnata $\mathrm{L}$. & Swamp Milkweed \\
\hline Asclepias tuberosa $\mathrm{L}$. & Butterfly Milkweed \\
\hline Bourreria succulenta Jacq. & Bahama Strongbark \\
\hline Byrsonima lucida (Mill.) DC. & Locustberry \\
\hline Cardiospermum corindum $\mathrm{L}$. & Balloonvine, Heartseed \\
\hline Chamaecrista fasciculata (Michx.) Greene & Partridge Pea \\
\hline Citharexylum spinosum $\mathrm{L}$. & Florida Fiddlewood \\
\hline Coccothrinax argentata (Jacq.)L.H. Bailey & Florida Silver Palm \\
\hline Colubrina asiatica (L.)Brongn. & Asian Nakedwood, Latherleaf \\
\hline Colubrina elliptica (Sw.)Brizicky \& W.L.Stern & Soldierwood \\
\hline Cordia sebestena $\mathrm{L}$. & Largeleaf Geigertree \\
\hline Croton linearis Jacquin & Pineland Croton \\
\hline Cynophalla flexuosa (L.) J. Presl & Bayleaf Capertree, Limber Caper \\
\hline Ficus aurea Nuttall & Strangler Fig \\
\hline Flaveria linearis Lag. & Narrowleaf Yellowtops \\
\hline Guaiacum sanctum L. & Holywood Lignumvitae \\
\hline Guapira discolor (Spreng.) Little & Beeftree, Blolly \\
\hline Jacquinia keyensis $\mathrm{Mez}$ & Joewood \\
\hline Lantana involucrata $\mathrm{L}$. & Buttonsage \\
\hline Neyraudia reynaudiana (Kunth) Keng ex Hitchc. & Burmareed, Silkreed \\
\hline Passiflora suberosa $\mathrm{L}$. & Corkystem Passionflower \\
\hline Physalis walteri Nutt. & Walter's Groundcherry \\
\hline Pithecellobium unguis-cati (L.) Benth. & Catclaw Blackbead \\
\hline Plumbago zeylanica $\mathrm{L}$. & Doctorbush \\
\hline Psychotria nervosa Swartz & Wild Coffee \\
\hline Ruta graveolens $\mathrm{L}$. & Rue \\
\hline $\begin{array}{l}\text { Senna mexicana var. chapmanni (Isely) H.S. Irwin } \\
\text { \& Barneby }\end{array}$ & Chapman's Wild Sensitive Plant \\
\hline Solidago sempervirens $\mathrm{L}$. & Seaside Goldenrod \\
\hline Varronia globosa Jacq. & Butterflybush, Curacao Bush \\
\hline Zamia integrifolia $\mathrm{L}$. & Coontie \\
\hline Zanthoxylum fagara (L.) Sarg. & Lime Pricklyash, Wild Lime \\
\hline
\end{tabular}


CHAPTER II

RISING SEAS IN THE FLORIDA KEYS: THE LAST REFUGE FOR THE FEDERALLY ENDANGERED SCHAUS' SWALLOWTAIL BUTTERFLY

(HERACLIDES ARISTODEMUS PONCEANUS) 


\section{ACKNOWLEDGEMENTS}

The authors would like to thank Tanjim Hossain and Suzanne Koptur for helpful suggestions on the manuscript, and John Koptur-Palenchar for assistance in obtaining obscure documents. We would like to thank Stephanie Garcia, Stefanie Moore, and Jay Sah for providing statistical consultation.

Dr. Kevin Whelan is the principal investigator for the Schaus' Swallowtail Habitat Enhancement Project. He collaborated with Tony Pernas, responsible for invasive plant species control in the national parks. Their dedication and continued services to the national parks' maintenance and integrity in south Florida and Caribbean provide enjoyment, education, and preservation for future generations. We would like to thank Biscayne National Park (BNP) and National Park Service personnel for providing access to the study sites (study numbers BISC-00041 and BISC-00046 under permits BISC2014-SCI-0031, BISC-2015-SCI-0023, and BISC-2016-SCI-0044). Craig Perry was instrumental in facilitating restoration site maintenance including data collection. Michael Foguer and Darlene Darrican provided additional support in data collection and site maintenance.

We are grateful to all volunteers from the Sierra Club, FIU, University of Miami, Doral High School, John Pennekamp State Park, BNP, and the NPS for their assistance in site preparation and maintenance. The Schaus' Swallowtail Habitat Enhancement Project was supported by the U.S. Fish and Wildlife Service. The FWS South Florida Coastal Program provided partial funding. Geographic Information System BNP spatial data were provided by personnel at NPS-SFCN. Florida International University GIS Center resources and staff were helpful in mapmaking and data analysis. This paper is 
contribution \# to the Tropical Biology Program at Florida International University, Miami, FL.

\begin{abstract}
The federally endangered Schaus' swallowtail butterfly (Heraclides aristodemus ponceanus) is restricted to intact subtropical dry forest habitats in the northern Florida Keys. Prime Schaus' swallowtail butterfly habitat is protected from anthropogenic harm in Biscayne National Park (BNP) and state parks in North Key Largo, Florida; however, sea level rise (SLR) ultimately might submerge the last remaining viable refugia. A habitat enhancement project in BNP designated four restoration sites at Elliott Key and Adams Key to increase the number of host plants (Amyris elemifera and Zanthoxylum fagara) specifically for the Schaus' swallowtail butterfly. Sea torchwood (A. elemifera) mortality was substantially higher than wild lime (Z. fagara) at the restoration sites. Predictor variables (canopy cover, elevation, relative soil depth) did a poor job in determining host plant mortality. Elevation had a significant effect on sea torchwood and wild lime growth in the restoration sites.

Spatial analyses were conducted to quantify dry forest habitat presence along elevation gradients in BNP and North Key Largo. Potential dry-forest habitat loss was then estimated based on imminent sea level rise (SLR) scenarios. Habitat was quantified for SLR increments of $25 \mathrm{~cm}$ up to $200 \mathrm{~cm}$. In the future, dry forest habitat will be particularly vulnerable at Adams Key. Biscayne National Park will lose more dry forest habitat faster than North Key Largo. If projected SLR follows the worst-case scenario (200 cm), only an estimated 7.47 ha of protected dry forest habitat will remain in BNP
\end{abstract}


and 202.89 ha in North Key Largo. Managed relocation to the mainland of Florida (historic range) or global policy changes to monumentally sequester atmospheric carbon dioxide should be contemplated for long-term Schaus' swallowtail butterfly preservation and other flora and fauna dependent on dry forest habitats in the northern Florida Keys.

Key Words: Amyris elemifera, climate change, Florida Keys, sea level rise, tropical dry forests

\section{INTRODUCTION}

Biscayne National Park (BNP) and North Key Largo are the last refugia for the federally endangered Schaus' swallowtail butterfly (Heraclides aristodemus ponceanus Schaus) in the United States (Emmel et al., 1988; Bibb and Hughes, 2007). Historically, the Schaus swallowtail butterfly inhabited subtropical dry forests in the Miami area south to Lower Matecumbe Key in the Florida Keys (Emmel et al., 1988). On the mainland of Florida (Miami area), subtropical dry forests were widespread and situated at higher elevations; however, anthropogenic modifications to the environment decimated viable habitat used by the Schaus' swallowtail butterfly, which was presumed extinct on the mainland in 1924 (Emmel et al., 1988). Subtropical dry forests were also expansive and thrived at higher elevations in the Florida Keys; consequently, these forests were developed and fragmented over time (Snyder et al., 1990; Strong and Bancroft, 1994; Noss et al., 1995). By 1984, the range of the Schaus' swallowtail butterfly was constricted to several large islands (Keys) in BNP and North Key Largo (Emmel et al., 1988). These forests are essential for the Schaus' swallowtail butterfly because their host 
plants, sea torchwood (Amyris elemifera L.; Figure 1) and wild lime (Zanthoxylum fagara

(L.) Sarg.), exists primarily in dry forest ecosystems (Rutkowski, 1971; Minno and Emmel, 1993). Both species of plants thrive in gaps and edges of subtropical dry forest habitat; however, sea torchwood frequently occurs in the interior of hammocks (Ray et al., 1998; Jameson, 2002). Sea torchwood, the preferred host plant for the Schaus' swallowtail butterfly, is a medium-sized ( $10 \mathrm{~m}$ tall) evergreen tree with compound leaves. Given the rapid sea level rise scenario of future climatic conditions, the remaining dry forest ecosystems in BNP and North Key Largo are vulnerable to extinction in the near future (Titus and Richman, 2001; Ross et al., 2009; Saha et al., 2011). Rising sea level presents an ominous, inevitable danger to the Schaus' swallowtail butterfly, which occupies a limited number of sites with suitable habitat in the northern Florida Keys (Bibb and Hughes, 2007).

Subtropical dry forest ecosystems are typically found in the interior or at the highest elevation in the Florida Keys (Ross et al., 1992; Karim and Main, 2009). Unfortunately, the Florida Keys are low-lying islands, thus preventing dry forest retreat to higher elevations as sea level continues to rise (Ross et al., 2003; Maschinski et al., 2011); therefore, dry forest ecosystems will transition to more salt-tolerant communities (Ross et al., 2009; Saha et al., 2011). The Florida Keys are the exposed remains of ancient coral reefs (Hoffmeister and Multer, 1968; Lidz and Shinn, 1991). The Keys form an archipelago positioned off the southern coast of Florida (Lidz and Shinn, 1991). The Florida Keys' tropical savanna climate invites a diverse array of plant species from the Caribbean reaching their northern limit in south Florida (Long, 1974; Tomlinson, 1986). Subtropical dry forests are dense, evergreen forests dominated by broad-leaved trees 
concentrated in the southern portion of Florida including the Florida Keys (Snyder et al., 1990; Ross et al., 1992; Gillespie, 2005). The assemblage of subtropical dry forests in the northern and middle Keys have limestone outcroppings, which are marine-based and classified as Key Largo limestone and partially covered with a shallow layer of endogenous soil (Hoffmeister and Multer, 1968, Horvitz and Koop, 2001; Armentano et al., 2003; Ross et al., 2003). The dry forests are known for their high species diversity (more than 120 plant species) that supports an equally diverse array of wildlife (Snyder et al. 1990; Gillespie, 2005).

Dry forest habitat in BNP is federally protected while $56 \%$ of dry forest habitat is protected in Key Largo (Karim and Main, 2009). North Key Largo protects 824.53 ha of dry forest in state parks (Dagny Johnson Key Largo Hammock Botanical State Park and Crocodile Lake National Wildlife Refuge), which is 199.47 less hectares than reported (1,024 ha) by Karim and Main (2009) indicating more habitat loss. Schaus' swallowtail butterfly sightings are periodically recorded in Key Largo; however, recent butterfly surveys have reported a substantial increase in Schaus' swallowtail butterfly observations (Minno, 2015). By far, the largest populations are sustained in BNP (Emmel et al., 1988; Minno, 2015). The largest extent of dry forest (703 ha) is protected in BNP and North Key Largo from development (Figure 2; Karim and Main, 2009). Scientists, staff, and volunteers from the University of Florida re-initiated the Schaus' Swallowtail CaptiveBreeding Program to augment butterfly populations (Daniels, 2014a; Schaus' Swallowtail Recovery Project, 2017). The majority of Schaus' swallowtail butterflies were released in BNP; nonetheless, some were released in North Key Largo (Minno, 2015). 
The Schaus' Swallowtail Habitat Enhancement Project through the National Park Service South Florida Caribbean Network (NPS-SFCN) was initiated to restore degraded habitat within prime Schaus' swallowtail territory at BNP by substantively increasing the number of host plants, mainly torchwood (Whelan, 2011; Whelan and Atkinson, 2015). Restored sites were previously overwhelmed with invasive plant species, particularly two species originating from the Old World: latherleaf (Colubrina asiatica (L.) Brongn.) and Burma reed (Neyraudia reynaudiana (Kunth) Keng ex Hitchc.). After the invasive plant species were removed, host and nectar plants, and a few native trees were planted in the restoration sites at Elliott Key and Adams Key. Increasing the number of resources (host and nectar plants) for butterflies can be a useful strategy to mitigate against habitat loss and generate corridors between suitable habitats (Pfitsch and Williams, 2009; Revathy et al., 2014; Shuey et al., 2016). The Schaus' swallowtail is threatened with extinction because it utilizes few host plants species restricted to subtropical dry forests in the Florida Keys (Rutkowski, 1971; Minno and Emmel, 1993; Bibb and Hughes, 2007). Sea torchwood flourish in subtropical and tropical dry forests (Lugo et al., 1978; Ross et al., 1992; Ray et al., 1998; Jameson, 2002). Subtropical dry forest ecosystems are not tolerant to long-term salt and brackish water flooding, acquire freshwater from the leaf litter and shallow layer of soil, and can tolerate mildly brackish groundwater (McGuire Jr and Brown, 1974; Sternberg et al., 2007); nonetheless, many plant species in dry forests inhibit photosynthesis when suitable water is unavailable (Lugo et al., 1978; Ross et al., 2003; Sternberg et al., 2007). 
Sea torchwood survivorship and growth were monitored from $2012-2017$ while wild lime survivorship and growth were monitored from 2012 - 2014 in the Schaus' Swallowtail Habitat Enhancement Sites in BNP.

The objectives of our study were to:

1) Track survivorship and growth of sea torchwood and wild lime planted in restoration sites at Elliott Key and Adams Key;

2) Quantify the extent of subtropical dry forest habitats in prime Schaus' swallowtail territory; and

3) Estimate dry-forest habitat loss due to future sea level rise.

\section{METHODS}

\section{$\underline{\text { Study Sites }}$}

Biscayne National Park (BNP) is located in southeast Florida and protects aquatic and terrestrial ecosystems. Ninety-five percent of the protected space is marine ecosystem; however, the remaining $5 \%$ includes mangrove and dry forest ecosystems. The greatest plant species diversity occurs in dry forest ecosystems in BNP (Long, 1974; Stalter et al., 1999). The primary and secondary host plants for Schaus' swallowtail butterflies, sea torchwood $\left(1^{\circ}\right)$ and wild lime (2०; Minno and Emmel, 1993; Jameson, 2002), predominately flourish in dry forest ecosystems (Rutkowski, 1971; Lugo et al., 1978; Jameson, 2002). The dry forest terrain is rocky (Key Largo limestone) with overlying leaf litter from trees that forms a shallow, organic layer of soil (Hoffmeister and Multer, 1968; Armentano et al., 2003; Ross et al., 2003). Naturally occurring sea torchwood were 
randomly selected and marked with a GPS unit at Elliott Key to determine their range of elevation. The islands in BNP can only be reached by watercraft, which offer some protection against negative human impacts such as illegal collection, pollution, pets, and negligence.

North Key Largo located south of BNP is close enough for Schaus' swallowtail butterflies to occasionally disperse between BNP and North Key Largo (Emmel et al., 1988). The rate of migration between sites is unknown; however, marked Schaus' swallowtail butterflies in BNP have been observed in dry forests at North Key Largo. Dry forest characteristics and dynamics in North Key Largo are similar to dry forests in BNP; although, dry forests are more mature with taller trees in North Key Largo (Ross et al., 1992).

\section{$\underline{\text { Schaus' Swallowtail Habitat Enhancement Sites }}$}

The duration of this study commenced in 2012 and ended in 2017. Volunteers and staff members at BNP and NPS-SCFN planted over 3,000 native plants in the restoration sites and along trails in the interior of Elliott Key during the wet seasons in 2012, 2013, and 2014 (Table 1; Whelan and Atkinson, 2015; Clayborn et al., 2017). In 2012, sea torchwood, wild lime, and nectar plant seedlings and native tree saplings were planted in restored sites and adjacent fringe areas (breezeway) at Elliott Key and Adams Key (Figure 3). Most of the plants at the sites were sea torchwood $(1,720)$ followed by 386 wild lime saplings (Table 1; Whelan and Atkinson, 2015). Sundry nectar plants and native trees were also planted in the restored sites and breezeways. All planted sea torchwood and wild lime saplings were labeled with aluminum tags for identification and 
spatially marked with a Trimble GPS unit (Figure 4). Collectively, there were two restored sites and two breezeways at Elliott Key and Adams Key. Additional sea torchwood was planted along established trails at Elliott Key the following years. Survivorship and stem elongation (growth) data for the sea torchwood planted along the trails are not reported in this paper.

Survivorship and stem elongation for sea torchwood were monitored from 2013 2017. Wild lime was monitored from 2013 - 2014. Due to logistical constraints during the fourth sampling period, a proportion of sea torchwood saplings were randomly sampled in the restored sites and breezeways to representatively assess survivorship and stem elongation. Survivorship was determined by sea torchwood and wild lime mortality (alive or dead); stem elongation measured the length of the stem from the base (ground) to the top (apical meristem) of the plant. Relative soil depth was measured with a sturdy, thin wire. The wire was pushed through the soil until it hit solid, hard substrate and could not go farther. Canopy cover, percent overstory, was measured with a densiometer one meter from the ground above the torchwood seedlings (Vora, 1988). Ground elevation for each plant and restoration site was extracted from elevation data derived from Light Detection and Ranging (LIDAR) data.

\section{$\underline{\text { Dry-Forest Habitat Loss }}$}

Biscayne National Park, and North Key Largo are low-lying islands with little relief from the surrounding sea (Snyder et al., 1990; Lidz and Shinn, 1991). Dry forests are found at the highest elevations in BNP and on North Key Largo (Ross et al., 1992). Vegetation cover data for BNP were acquired from the Biscayne National Park, Florida, 
Vegetation Mapping Project, 2006-2009 (South Florida Terrestrial Ecosystems Lab, 2008), and for Key Largo from Monroe County's 2006 "Land Cover dataset for the Florida Keys" (Photo Science Inc. 2009). South Florida vegetation maps refer to dry forest as hardwood hammock, therefore, classes with the hardwood hammock label including scrubland hammock were re-classified as subtropical dry forest. Vegetationcover classes that corresponded to subtropical dry forests in the BNP Level 3 data layer were 'Hammock Forest', 'Coastal Hardwood Hammock', and 'Coastal Hardwood Shrubland'. The corresponding class in the Monroe County dataset layer was 'Hammock'.

Elevation gradient analysis was conducted to estimate dry forest cover distribution along the elevation gradient for different sub-regions of BNP and North Key Largo. Other dry forest patches south of North Key Largo, but still on Key Largo were also included as separate sub-region in this analysis. The extent of dry forest habitat was spatially stratified for eight sites in BNP and 4 sites on Key Largo. The elevation data used for this analysis was the 2008 National Elevation Dataset (NED) courtesy of the U.S. Geological Survey.

Potential dry forest habitat loss due to SLR within BNP and Key Largo was estimated for incremental SLR at intervals of $25 \mathrm{~cm}$. To estimate the elevation range of extant dry forest habitat within the study area, the range of dry forest habitat elevation was estimated with the threshold of the upper $95^{\text {th }}$ percentile of elevations extracted for dry forest from the NED. For conservative estimates of future dry forest habitat loss, this elevation threshold was applied to elevations for each SLR scenario. The habitat analyses were conducted in R (R Core Team, 2017). 


\section{DATA ANALYSIS}

Before statistical analyses were performed on the dataset, extreme outliers were removed from the response and predictor variables. Extreme outliers may have been measurement miscalculations in the field. Nonparametric tests were performed because several predictor and dependent variables violated the normal (Gaussian) distribution assumption. Nonparametric tests are distribution-free tests and considered less powerful than parametric tests when sample sizes are small (Fisher and van Belle, 1993); accordingly, nonparametric tests are nearly as powerful as parametric tests when sample sizes are large (Conover and Iman, 1981; Fisher and van Belle, 1993).

The Kruskal-Wallis $\mathrm{H}$ test was used to compare differences between restored and breezeway sites in BNP. Canopy cover, elevation, and soil depth were tested individually as dependent variables with site locations listed as fixed factors. If a significant difference was reported among groups, post hoc pairwise comparisons were then applied to determine which groups differed from each other.

A binary logistic regression was performed to test the effects of multiple predictor variables (elevation, relative soil depth, and canopy cover) against the response variable (survivorship). Sea torchwood survivorship followed a binomial distribution (alive or dead) and was recorded in 2013, 2014, and 2017 while wild lime survivorship was in 2013 and 2014.

Stem elongation was standardized to account for growth over time (rate of change) by using the formula: 


$$
\text { Annual Stem Elongation }=\frac{\text { Final Height }- \text { Initial Height }}{\text { Initial Height } * \text { Number of Days }} * 365.25 \text { Days }
$$

A linear regression model was used to analyze the effects of multiple predictor variables (elevation, relative soil depth, and canopy cover) against the response variable (annual stem elongation). A stepwise regression was applied to remove the weakest correlated variables, if any. Collinearity diagnostics were performed to detect multicollinearity between predictor variables during the linear rank and binary logistic regression analyses. Residual plots were inspected to determine if assumptions were met.

A receiver operating characteristic (ROC) curve analysis was used to assess the performance of diagnostic decision-making processes between host plant survivorship (sea torchwood and wild lime) and elevation, canopy cover, and soil depth (Hosmer and Lemeshow, 2000; Sah et al., 2010). We used IBM SPSS version 19 (SPSS, Chicago, IL, USA) to detect extreme outliers and perform Kruskal-Wallis H tests, binary logistic regression, stepwise linear regression, and ROC curve analyses.

\section{RESULTS}

Sea torchwood and wild lime data, collected by staff and volunteers at NPS-SFCN, were organized and examined using the program Microsoft Excel 2016. The number of sea torchwood and wild lime reported in Whelan and Atkinsons (2015) showed great fidelity to the dataset used in this study (compare numbers in Table 1 to Table 2). 


\section{$\underline{\text { Natural distribution of sea torchwood }}$}

Sea torchwood naturally occurs along the exterior and interior of tropical dry forests (Ray et al., 1998; Jameson, 2002). Sea torchwood, on average, occurred $1.10 \mathrm{~m}$ above sea level at Elliott Key $(n=54, \bar{x}=1.10 \mathrm{~m}, \mathrm{~s}=0.28$; Figure 5). Spatial analysis of dry forest habitats in the northern Florida Keys revealed $95 \%$ of dry forest habitat occurred $0.30 \mathrm{~m}$ and higher above sea level.

\section{$\underline{\text { Study Sites }}$}

Canopy cover (percent overstory) was significantly different at each site except the breezeway site at Elliott Key and restored site at Adams Key, which were not significantly different from each other (Table 3; Figure 5). Canopy cover above sea torchwood was highest in the main restoration site at Elliott Key and lowest along the breezeway site at Adams Key (Table 4; Figure 6). Sea torchwood seedlings were planted at significantly higher elevations in the main restoration site at Elliott Key than the other sites (Tables 3 and 4; Figure 7). Pairwise comparison analyses between sites revealed the elevational distribution of sea torchwood planted at each site was significantly different (Table 3; Figure 7). Soil depth was significantly different at each site (Table 3; Figure 8). The distribution of soil depth for each sea torchwood was highest in the main restoration site at Adams Key and lowest along the breezeway at Elliott Key (Table 4; Figure 8). Annual sea torchwood stem elongation (growth) was significantly higher in the in the main restoration site at Elliott Key during 2013, 2014, and 2017; Tables 5 and 6; Figures 9, 10, and 11). Pairwise comparison analyses between sites revealed annual sea torchwood growth at one site was significantly different from each other site (Table 5; 
Figure 9, 10, and 11). The worst site for sea torchwood growth was along the breezeway at Adams Key during 2013, 2014, and 2017 (Table 6; Figures 9, 10, 11). Annual sea torchwood growth was much higher at Elliott Key than Adams Key each year (Tables 6).

The elevational distribution of wild lime planted in the main restoration site at Elliott Key was significantly higher than the other sites (Tables 3 and 4; Figure 12). Pairwise comparison analyses between sites revealed the elevational distribution of wild lime planted at each site was significantly different except the breezeway site at Elliott Key and main restoration site at Adams Key (Table 4; Figure 12). Annual wild lime stem elongation (growth) was significantly higher in the main restoration site at Elliott Key during 2013 and 2014 (Tables 5 and 6; Figures 13 and 14). Pairwise comparison analyses between sites revealed annual wild lime growth at a site was significantly different from each other site, except the breezeway site at Elliott Key and main restoration site at Adams Key which were not distinguished from one another (Table 5; Figures 13 and 14). The worst site for wild lime growth occurred along the breezeway at Adams Key during 2013 and 2014 (Table 6; Figures 13 and 14). Annual wild lime growth increased in 2014 at all sites (Table 6).

\section{$\underline{\text { Host plant survivorship }}$}

Host plant survivorship over time at each site was quantified to track mortality from 2013 to 2017 for sea torchwood and 2013 to 2014 for wild lime. In 2013, sea torchwood survivorship was highest along the breezeway (71.4\%) and restored (64.5\%) sites at Elliott Key, and lowest in the restored (56.7\%) and breezeway (34.3\%) sites at Adams Key (Table 2). Roughly 4 years later (2017), sea torchwood survivorship remained the 
highest in the breezeway (60.8\%) and restored (58.1\%) sites at Elliott Key compared to the restored (45\%) and breezeway (6.9\%) sites at Adams Key (Table 2). Sea torchwood mortality was particularly severe in the breezeway site (6.9\%) at Adams Key, where only 19 individuals survived out of 274. Overall, combined sea torchwood survivorship at all sites dropped from $59.6 \%$ (2013) to $47.5 \%$ (2017; Table 2).

Wild lime survivorship was much higher at all sites in 2013 and 2014 than sea torchwood. In 2013, wild lime survivorship was highest in the restored sites at Elliott Key (98.3\%) and Adams Key (89.2\%) and lowest along the breezeway sites at Elliott Key (89.2\%) and Adams Key (87.5\%; Table 2). In 2014, wild lime survivorship was highest in the restored sites at Elliott Key (98.3\%) and Adams Key (92.3\%) compared to the breezeway sites at Elliott Key (87.3\%) and Adams Key (84.4\%; Table 2). Overall, combined wild lime survivorship at all sites declined only slightly: $1.2 \%$ from $92.7 \%$ (2013) to 91.5\% (2014; Table 2). Although the 2013 to 2014 decline in sea torchwood survivorship was minimal (2\%), it declined dramatically from 2013 to 2017 (12.1\%).

\section{Host plant stem elongation (growth) and mortality}

Ground elevation was an important, consistent variable for sea torchwood annual stem elongation during the stepwise regression analysis each year $(2013,2014$, and 2017). Canopy cover was included in the analysis in 2014. Relative soil depth was included in the analysis in 2017. Elevation, canopy cover, and soil depth all had significant effects on sea torchwood growth; however, most of the variation accounted for sea torchwood was attributed to ground elevation (Table 7). The adjusted co-efficient of determination $\left(\mathrm{r}^{2}\right)$ value decreased each year $\left(2013\right.$, adj. $\mathrm{r}^{2}=0.296 ; 2014$, adj. $\mathrm{r}^{2}=$ 
0.281; 2017, adj. $\mathrm{r}^{2}=0.248$; Table 7). Elevation had a significant effect on wild lime growth both years $(2013,2014)$. The adjusted co-efficient of determination $\left(\mathrm{r}^{2}\right)$ value increased each year $\left(2013\right.$, adj. $r^{2}=0.255 ; 2014$, adj. $r^{2}=0.279$; Table 7).

The coefficient of determination $\left(\mathrm{r}^{2}\right)$ value cannot be calculated for logistic regression models; however, two pseudo $r^{2}$ values can be calculated. The Cox and Snell $r^{2}$ and Nagelkerke $r^{2}$ values attempt to measure the amount of variation accounted for in the model. The Hosmer-Lemeshow goodness of fit test was applied to test how well the data fit the model. In 2013, the best fit model for sea torchwood included ground elevation and relative soil depth and excluded canopy cover. In 2014, the best fit model again only included ground elevation and relative soil depth and excluded canopy cover. In 2017, the best fit model included relative soil depth, canopy cover, and ground elevation. The Cox and Snell $r^{2}\left(2013, r^{2}=0.063 ; 2014, r^{2}=0.081 ; 2017, r^{2}=0.114\right)$ and Nagelkerke $r^{2}$ $\left(2013, \mathrm{r}^{2}=0.086 ; 2014, \mathrm{r}^{2}=0.109 ; 2017, \mathrm{r}^{2}=0.153\right)$ values increased each year for sea torchwood survivorship (Table 8). The predictor variables applied to the best fit models for each year were significant in sea torchwood survivorship; consequently, the effect of the predictor variables increased each year. Elevation was the only predictor variable applied to wild lime mortality in the logistic regression model. Based on the HosmerLemeshow goodness of fit test, ground elevation did not fit the model. The Cox and Snell $r^{2}\left(2013, r^{2}=0.034 ; 2014, r^{2}=0.084\right)$ and Nagelkerke $r^{2}\left(2013, r^{2}=0.041 ; 2014, r^{2}=\right.$ 0.093) values were negligible for wild lime survivorship (Table 8). 


\section{$\underline{\text { Receiver operating characteristic (ROC) curve }}$}

Accuracy of the ROC curve is measured by the area under the curve (AUC). The AUC grade system: $>0.90-1.00=\operatorname{excellent}(\mathrm{A}),>0.80-0.90=\operatorname{good}(\mathrm{B}),>0.70-0.80$ $=$ fair $(C),>0.60-0.70=$ poor $(D), 0.50-0.60=$ fail $(F)$. The area under the curve (AUC) for canopy cover and relative soil depth was poor for sea torchwood survivorship each year (Table 9). The AUC value was poor for elevation during 2013 and 2014 and fair for elevation in 2017 (Table 9). Wild lime AUC values were fair for elevation during 2013 and 2014 (Table 9). The diagnostic tests (ROC curve) for sea torchwood survivorship were weak for canopy cover and relative soil depth and slightly better for elevation in 2017. The diagnostic tests for elevation were better for wild lime survivorship in 2013 and 2014 (Table 9).

\section{Dry forest habitat loss in Biscayne National Park and Key Largo}

The Schaus' Swallowtail Habitat Enhancement Project restored subtropical dry forest habitat at Elliott Key and Adams Key in BNP. Currently, Elliott Key has the largest extent of dry forest habitat in BNP (426.83 ha; Table 10). Consequently, Adams Key has much less dry forest habitat (12.60 ha; Tables 10). Old Rhodes Key is another large island in BNP with the second largest extent of dry forest habitat (122.38 ha; Table 10). Schaus' swallowtail butterflies also inhabit subtropical dry forest habitats in North Key Largo (Minno, 2015). Protected dry forest habitat (Palo Alto Key, Broad Key, Linderman Key, Crocodile Lake National Wildlife Refuge, and Key Largo Hammock State Botanical Site) has declined in Key Largo (1,024.00 ha > 824.53 ha) since Karim and Main (2009) conducted their study (Table 10). Subtropical dry forest habitat extends 
south past North Key Largo; however, no recent verified Schaus' swallowtail sightings have been reported. Dry forest habitat south of North Key Largo in Key Largo is more fragmented and adjacent to more commercial and residential development. Key Largo Hammock State Botanical Site has the largest extent of dry forest habitat in North Key Largo (542.98 ha; Table 10). Collectively, dry forest habitats combined in Key Largo south of North Key Largo covers 488.34 ha (Table 10). A large section of dry forest remains unprotected (150.71 ha) in North Key Largo adjacent to the state protected parks (Crocodile Lake National Wildlife Refuge and Key Largo Hammock State Botanical Site; Table 10).

Collectively, the islands in BNP are lower in elevation than Key Largo, which means they are more vulnerable to the imminent threat of SLR. Ninety-five percent dry forest habitats range from $0.3 \mathrm{~m}$ above sea level to the highest elevation in BNP and Key Largo. Remaining dry forest habitat in BNP and Key Largo based on $0.25 \mathrm{~cm}$ increments of SLR up to $200 \mathrm{~cm}$ is displayed in Table 10 and color-coded in Figures 15-18. At the SLR 100 cm mark, 32\% of dry forest habitat remains in BNP while $65 \%$ remains in Key Largo (Table 10). At the SLR $200 \mathrm{~cm}$ mark, $1 \%$ of dry forest habitat remains in BNP while 29\% remains in Key Largo (Table 10). Dry forest habitat in Crocodile Lake National Wildlife Refuge occurs at higher elevations and potentially will not succumb to SLR as quickly as the other sites (Table 10; Figure 18). Sea torchwood and wild lime planted at Adams Key are extremely vulnerable to rising seas (Table 10; Figure 16). 


\section{DISCUSSION}

The remaining viable habitat for the Schaus' swallowtail butterfly exists in BNP and North Key Largo (Bibb and Hughes, 2007; Minno, 2015). Anthropogenic subtropical dry habitat loss is no longer a major threat because of federal protection in BNP and state protection in North Key Largo. However, the Schaus' swallowtail butterfly's range is confined to islands vulnerable to severe storms and rising seas. Marine and brackish water encroachment on land and within the ground water table will have deleterious effects on dry forest habitats in the Florida Keys (McGuire Jr and Brown, 1974; Sternberg et al., 2007; Ross et al., 2009). More importantly, the imperiled Schaus’ swallowtail butterfly's host plants that exist in dry forest ecosystems will be threatened by severe storms and SLR. Restoration projects that maintain the integrity of dry forest ecosystems are essential for the Schaus' swallowtail butterfly (Bibb and Hughes, 2007).

In 2013, ground elevation had a significant effect on sea torchwood growth and accounted for $29.6 \%$. In 2014, ground elevation and canopy cover had a significant effect and accounted for $28.1 \%$ of the variation. In 2017, ground elevation and relative soil depth had a significant effect and accounted for $24.8 \%$ of the variation (Table 7). The effect of these variables was more pronounced during the first year than the last year of data collection. An increase in ground elevation promoted faster sea torchwood growth each year, while a decrease in canopy cover (2014) and relative soil depth (2017) contributed to faster sea torchwood growth. Ground elevation also had a significant and strong influence $(2013,25.3 \% ; 2014,27.9 \%)$ on wild lime stem elongation each year (Table 7). An increase in elevation promoted faster wild lime growth. Access to water and water availability are two major factors (not measured during this study) that 
dramatically affect plant growth. Sea torchwood and wild lime thrive in dry forest ecosystems with defined wet and dry seasons. However, there is more protection from droughts in the forest's interior than along the exterior or outside (Lugo et al., 1978; Ross et al., 2003).

Ground elevation, relative soil depth, and canopy cover were significant factors in sea torchwood survivorship; however, the variables had a small effect on survivorship the first two years (Table 8). In 2017, all three variables had a modest effect on sea torchwood survivorship (Table 8). Elevation was not a relevant variable that influenced wild lime survivorship (Table 8). Wild lime was more robust with much less mortality than sea torchwood during 2013 and 2014 (Table 2, 8). Overall, the Cox and Snell $\mathrm{r}^{2}$ and Nagelkerke $r^{2}$ values were low for both host plant species, which means other unknown factors contributed to their success or demise. Belowground factors such as soil salinity, access to the water table, water table quality (fresh, brackish, or marine), and moisture present in the leaf litter also contribute to sea torchwood and wild lime survivorship (Ross et al., 2003; Sternberg et al., 2007). The ROC curve diagnostic tests were poor models for canopy cover and relative soil depth. Sea torchwood survivorship AUC value was fair (71.6\%) for elevation in 2017 (Table 9). Wild lime survivorship AUC values were also fair for elevation $(75.0 \%, 2013 ; 73.8 \%, 2014$; Table 9). Ideally, if the ROC curve results would have been excellent $(>0.90-1.00)$ or good $(>0.80-0.90)$, the predictor variables (logistic regression) probably would have had a stronger influence on sea torchwood and wild lime survivorship.

Sea torchwood and wild lime are more vulnerable to SLR at Adams Key than Elliott Key. There is much less available dry forest habitat at Adams Key than Elliott Key 
(Table 10; Figures 15, 16). Dry forest habitat is lower in elevation at Adams Key than Elliott Key. Old Rhodes Key and Totten Key are large islands with large intact dry forest habitats and higher elevation than Adams Key (Table 10; Figures 16, 17); however, difficult access and terrain prevent consistent annual Schaus's swallowtail butterfly surveys. We know Elliott Key is considered the source island for the Schaus's swallowtail butterfly (Emmel, 1988; Minno, 2015); however, Old Rhodes Key and Totten Key should be surveyed to determine if populations of Schaus' swallowtail butterflies thrive there, including the amount of available resources for them.

The imminent threat of SLR is problematic for the Schaus' swallowtail butterfly if dry forest habitat is drastically reduced. Fortunately, Key Largo has more available dry forest now and will not succumb to SLR as fast as the islands in BNP (Table 10; Figure 18). Dry forest habitat loss, based on gradual SLR, indicates ecosystems will shift to more salt-tolerant plant species at lower elevations while dry forest plant species should continue to survive until there is no more available terrain to avoid the onslaught of SLR (Ross et al., 2009). Schaus' swallowtail butterfly host plants are not salt-tolerant species; therefore, as dry forest ecosystems disappear so will sea torchwood and wild lime. North Key Largo is connected to the mainland of southeast Florida by bridges; however, mosquitoes and tough terrain keep most people from exploring the dry forests' interior. There is more potential to maintain Schaus' swallowtail butterfly populations in Key Largo as insurance against populations in BNP. All unprotected dry forest habitat in Key Largo should be purchased and maintained as a functioning dry forest ecosystem.

Pulse events such as extreme high tides, severe storms, and storm surges from hurricanes (Williams et al., 2003) were not accounted for in the spatial analysis. 
Nonetheless, these events can drastically modify landscapes (Williams et al., 2003; Park et al., 2011; Park et al., 2017). Recent modeled SLR projections (by 2100) range from 0.2 $\mathrm{m}$ to $2.0 \mathrm{~m}$ (Melillo et al., 2014). If projected SLR follows the worst-case scenario (2.0 m), only an estimated 7.47 ha of protected dry forest habitat will exist in BNP and 202.89 ha in North Key Largo (Palo Alto Key, Broad Key, Linderman Key, Crocodile Lake National Wildlife Refuge, and Key Largo Hammock State Botanical Site; Table 10). We predict significant amounts of subtropical dry forest habitats will disappear and transition to habitats with more salt-tolerant plant species (Ross et al., 2009; Maschinski et al., 2011; Saha et al., 2011). Consequently, many organisms, including the Schaus's swallowtail butterfly, will disappear if suitable habitat becomes nonexistent (Ross et al., 2009; Minteer and Collins, 2010; Maschinski et al., 2011).

\section{CONCLUSION}

The protection of natural habitats and restoration of degraded sites are two of the many solutions to stymie species extinctions (Saunders and Hobbs, 1989; Ross et al., 2009). Currently, BNP remains a stronghold for populations of Schaus'swallowtail butterflies (Minno, 2015). Contiguous subtropical forest habitat protected against anthropogenic disturbances provide sufficient resources for Schaus' swallowtail butterflies to persistent. Host plant habitat enhancements and captive-bred butterfly

releases have supplemented wild populations (Minno, 2015; Whelan and Atkinson, 2015; Schaus' Swallowtail Restoration Project, 2017). The population in North Key Largo appears to be rebounding: butterfly surveyors reported no Schaus' swallowtail sightings from 2012 - 2013 (Daniels, 2014a, b), but 60 were sighted in North Key Largo in 2015, 
36 in 2016, and 58 in 2017 (Minno, 2015; L. Evans pers. comm). Despite federal and state protection of subtropical dry forest habitat in BNP and North Key Largo (Karim and Main, 2009), the more ominous threat of SLR will diminish dry forest habitat over time (Williams et al., 2003; Ross et al., 2009; Saha et al., 2011). The amount of suitable habitat required for the Schaus' swallowtail butterfly to persist is unknown and difficult to assess because populations often experience highly variable fluctuations (Bibb and Hughes, 2007; Minno, 2015). Habitat loss because of rising seas will undoubtedly have deleterious effects on Schaus' swallowtail butterfly populations.

Continued survival of Schaus' swallowtail butterflies depends on national and international policies that advance aggressive climate change mitigation to thwart SLR (United Nations, 2015), which also benefit Florida and other coastal communities. Other Schaus' swallowtail butterfly management considerations include managed relocation to historic ranges on the mainland of Florida (assisted migration) or permanent, captive colonies in museums, zoos, or other trusted facilities (Minteer and Collins, 2010; Maschinski et al., 2011). Given the imminent threat of SLR in the future, subtropical dry forests in the BNP and North Key Largo will decline; however, other mountainous Caribbean islands such as Cuba, Hispaniola, and Puerto Rico provide sanctuary for butterflies closely-related to Schaus' swallowtail butterflies (Heraclides aristodemus ponceanus). Heraclides aristodemus temenes (Cuba) and Heraclides aristodemus aristodemus (Hispaniola and Puerto Rico) are conspecific subspecies and share similar characteristics: (1) they occupy expansive tropical dry forest habitat with limestone outcroppings, (2) consume sea torchwood, wild lime, and other closely-related rutaceous species, and (3) have one to two flight seasons each year (Schwartz, 1989; Smith et al., 
1994; Hernández, 2004). The presence of Heraclides aristodemus subspecies on islands less threatened by SLR presents an optimistic future for Heraclides aristodemus species overall.

\section{LITERATURE CITED}

Armentano T.V., Jones, D.T., Ross, M.S., and Gamble, B.W. 2003. Pp. 225-282, In Sklar, F.H., and Van Der Valk, A. (Eds). Vegetation pattern and process in tree islands of the southern Everglades and adjacent areas. Tree islands of the Everglades. Kluwer Academic Publishers, Dordrecht, The Netherlands.

Bibb, K., and Hughes, P. 2007. United States Fish and Wildlife Service Schaus Swallowtail butterfly (Heraclides aristodemus ponceanus) 5-Year review: Summary and evaluation. United States Fish and Wildlife Service, Southeast Region, South Florida Ecological Services Office, Vero Beach, Florida. Available online at http://ecos.fws.gov/docs/five_year_review/doc1983.pdf. Accessed 12 January 2017.

Clayborn, J., Koptur, S., O’Brien, G., Whelan, K.R.T. 2017. The Schaus swallowtail habitat enhancement project: An applied service-learning project continuum from Biscayne National Park to Miami-Dade County public schools. Southeastern Naturalist, 16(10): 26-46.

Conover, W.J., and Iman, R.L. 1981. Rank transformation as a bridge between parametric and nonparametric statistics. The American Statistician, 35(3): 124-129.

Daniels, J.C. 2014a. Conservation matters: Status and conservation of the federally endangered Schaus swallowtail butterfly. News of the Lepidopterists' Society 56: $138-139$.

Daniels, J.C. 2014b. Occurrence, distribution and ecological studies of the endangered Schaus' Swallowtail butterfly. A report to the U.S. Fish and Wildlife Service and Imperiled Butterflies of South Florida Workgroup. 24 pp.

Emmel, T.C., Minno, M.C., Nation, J.L. Jr, Eliazar, P.J., Cotter, A.J., Bustamante, E.G., Ritland, D.B., Goode, M.R. 1988. Habitat requirements and status of the endemic Schaus' swallowtail in the Florida Keys. Final Project Report, Project Number GFC86-023. Submitted to Florida Game and Fresh Water Fish Commission Division of Wildlife Nongame Wildlife Section, Tallahassee, Florida.

Fisher, L.D., and van Belle, G. 1993. Biostatistics: A Methodology for the Health Sciences. John Wiley \& Sons, Inc., New York, USA. 
Gillespie, T.W. 2005. Predicting woody-plant species richness in tropical dry forests: A case study from South Florida, USA. Ecological Applications, 15(1): 27-37.

Hernández, L.R. 2004. Field Guide of Cuban - West Indies Butterflies. Ediluz, Maracaibo, Venezuela.

Horvitz, C.C., and A. Koop. 2001. Removal of nonnative vines and post-hurricane recruitment in tropical hardwood forests of Florida. Biotropica 33: 268-281.

Hoffmeister, J.E. and Multer, H.G. 1968. Geology and origin of the Florida Keys. Geological Society of America Bulletin, 79(11): 1487-1502.

Hosmer, D.W., and Lemeshow, S. 2000. Applied Logistic Regression ( $2^{\text {nd }}$ edition). John Wily \& Sons, New York, NY.

Jameson, A. 2002. Host Plants and Habitats of the Schaus Swallowtail Butterfly (Papilio Aristodemus Ponceanus). M.S. Thesis. University of Miami, Coral Gables, FL. 51 pp.

Karim, A., and Main, M.B. 2009. Habitat fragmentation and conservation strategies for a rare forest habitat in the Florida Keys archipelago. Urban ecosystems, 12(3): 359-370.

Lidz, B.H., and Shinn, E.A. 1991. Paleoshorelines, reefs, and a rising sea: South Florida, USA. Journal of Coastal Research, 7(1): 203-229.

Long, R.W. 1974. Origin of the vascular flora of southern Florida. In: Gleason, P.J. (ed) Environments of south Florida: past and present. Miami Geological Society, Miami, FL, pp. 28-36.

Lugo, A.E., Gonzalez-Liboy, J.A., Cintron, B., and Dugger, K. 1978. Structure, productivity, and transpiration of a subtropical dry forest in Puerto Rico. Biotropica, 10(4): 278-291.

Maschinski, J., Ross, M.S., Liu, H., O’Brien, J., von Wettberg, E.J., and Haskins, K.E. 2011. Sinking ships: conservation options for endemic taxa threatened by sea level rise. Climatic Change, 107: 147-167.

McGuire Jr, R.J., and Brown, L.N. 1974. A phytosociological analysis of two subtropical hammocks on Elliott Key, Dade County, Florida. Journal of the Elisha Mitchell Scientific Society, 90(4): 125-131.

Melillo, J.M., Richmond, T.C., and Yohe, G.W. (Eds.) 2014. Climate Change Impacts in the United States: The Third National Climate Assessment, U.S. Global Change Research Program, 841 pp. 
Minno, M.C. and Emmel, T.C. 1993. Butterflies of the Florida Keys. Scientific Publishers, Gainesville, FL.

Minno, M.C. 2015. 2015 Schaus' swallowtail (Heraclides aristodemus ponceanus) monitoring on Key Largo, Florida. Southern Lepidopterists' News, 37(3): 106-107.

Minteer, B.A., and Collins, J.P. 2010. Move it or lose it? The ecological ethics of relocating species under climate change. Ecological Applications, 20(7): 1801-1804.

Noss R.F., Roet E.T. III, and Scott J.M. 1995. Endangered ecosystems of the United States: A preliminary assessment of loss and degradation. Biological Report 28. US Department of the Interior, National Biological Service, Washington D.C

Park, J., Obeysekera, J., Irizarry, M., Barnes, J., Trimble, P., and Park-Said, W. 2011. Storm surge projections and implications for water management in South Florida. Climatic change, 107(1): 109-128.

Park, J., Stabenau, E., Redwine, J., and Kotun, K. 2017. South Florida's Encroachment of the Sea and Environmental Transformation over the 21st Century. Journal of Marine Science and Engineering, 5(3):31.

Pfitsch, W.A., and Williams, E.H. 2009. Habitat restoration for lupine and specialist butterflies. Restoration Ecology, 17(2): 226-233.

Photo Science, Inc. 2009. MonroeGISdata.DBO.E_Habitat_2009.

R Core Team. 2017. R: A language and environment for statistical computing. R Foundation for Statistical Computing, Vienna, Austria. http://www.R-project.org/.

Ray, G.J., Dallmeier, F., and Comiskey, J.A. 1998. The structure of two subtropical dry forest communities on the island of St John, US Virgin Islands. Pp. 367-384, In Dallmeier, F., and Comiskey, J.A. (Eds.). Forest Biodiversity in North, Central, and South America, and the Caribbean: Research and Monitoring, Man and the Biosphere Series. Vol. 21. UNESCO and the Parthenon Publishing Group, Camforth, Lancashire, UK.

Revathy, V.S., Mathew, G., and Narayanankutty, T.P. 2014. Role of recreated habitats in butterfly conservation: A case study at Thenmala, Kerala, India. International Journal of Tropical Insect Science, 34(4): 287-295.

Ross, M.S., O'Brien, J.J., and Flynn, L.J. 1992. Ecological site classification of Florida Keys terrestrial habitats. Biotropica, 24(4): 488-502. 
Ross, M.S., Coultas, C.L., and Hsieh, Y.P. 2003. Soil-productivity relationships and organic matter turnover in dry tropical forests of the Florida Keys. Plant and Soil, 253(2): 479-492.

Ross, M.S., O’Brien, J.J., Ford, R.G., Zhang, K., and Morkill, A. 2009. Disturbance and the rising tide: the challenge of biodiversity management on low-island ecosystems. Frontiers in Ecology and the Environment 7(9): 471-478.

Rutkowski, F. 1971. Observations on Papilio aristodemus ponceanus (Papilionidae). Journal of Lepidopterist Society, 25: 126-136.

Sah, J.P., Ross, M.S., Snyder, J.R., and Ogurcak, D.E. 2010. Tree mortality following prescribed fire and a storm surge event in slash pine (Pinus elliottii var. densa) forests in the Florida Keys, USA. International Journal of Forestry Research, 204795: 13 pp.

Saha, A.K., Saha, S., Sadle, J., Jiang, J., Ross, M.S., Price, R.M., Sternberg, L.S. and Wendelberger, K.S. 2011. Sea level rise and South Florida coastal forests. Climatic Change, 107(1): 81-108.

Saunders, D., and R. Hobbs. 1989. Corridors for conservation. New Scientist, 121: 63-68.

Schaus' Swallowtail Restoration Project. 2017. In Florida Museum University of Florida. Retrieved September 19, 2017, from https://www.floridamuseum.ufl.edu/museumvoices/special-projects-lab/schaus-swallowtail-restoration-project/.

Schwartz, A. 1989. The Butterflies of Hispaniola. University Press of Florida, Gainesville, Florida.

Shuey, J., Jacquart, E., Orr, S., Becker, F., Nyberg, A., Littiken, R., Anchor, T., and Luchik, D. 2016. Landscape-scale response to local habitat restoration in the regal fritillary butterfly (Speyeria idalia) (Lepidoptera: Nymphalidae). Journal of Insect Conservation, 20(5): 773-780.

Smith, D.S., Miller, L.D., and Miller, J.Y. 1994. The Butterflies of the West Indies and South Florida. Oxford University Press. New York.

Snyder, J.R., Herndon, A. and Robertson, W.B., Jr. 1990. South Florida rockland. In: Myers, R.L. and Ewel, J.J. (eds) Ecosystems of Florida. University of Central Florida Press, Orlando, FL, pp. 230-277.

South Florida Terrestrial Ecosystems Lab, Southeast Environmental Research Center at Florida International University. 2008. Biscayne National Park, Florida, Vegetation Mapping Project, 2006-2009. 
Stalter, R., Tamory, J., Lynch, P., and Lockwood, B. 1999. The vascular flora of Biscayne National Park, Florida. SIDA, Contributions to Botany, 18(4): 1207-1226.

Sternberg, L.D.S.L., Teh, S.Y., Ewe, S.M., Miralles-Wilhelm, F., and DeAngelis, D.L. 2007. Competition between hardwood hammocks and mangroves. Ecosystems, 10(4): 648-660.

Strong, A.M., and Bancroft, G.T. 1994. Patterns of deforestation and fragmentation of mangrove and deciduous seasonal forests in the upper Florida Keys. Bulletin of Marine Science 54(3): 795-804.

Titus, J.G., and Richman, C. 2001. Maps of lands vulnerable to sea level rise: modeled elevations along the U.S. Atlantic and Gulf coasts. Climate Research, 18: 205-228.

Tomlinson, P.B. 1986. The biology of trees native to tropical Florida. Harvard University, Cambridge, Massachusetts.

United Nations. 2015. Paris Agreement. Accessed September 20, 2017 https://unfccc.int/files/essential_background/convention/application/pdf/english_paris _agreement.pdf.

Vora, R.S. 1988. A comparison of the spherical densiometer and ocular methods of estimating canopy cover. The Great Basin Naturalist, 48(2): 224-227.

Whelan, K.R.T., and Atkinson, A. 2015. Schaus Swallowtail Butterfly (Heraclides aristodemus ponceanus) Habitat Enhancement in Biscayne National Park. South Florida Coastal Program Project Progress Report. NPS/SFCN. National Park Service, Palmetto Bay, Florida. 24 pp.

Whelan, K. R. T., Ruiz, P.L., Shamblin, R.B., Houle, P.A., Ross, M.S., Atkinson, A.J., Patterson, J.M., and Alonso, J. 2013. Biscayne National Park Vegetation Map Project Report [and associated files]. Natural Resource Technical Report. NPS/SFCN/NRTR - 2013/774. National Park Service. Fort Collins, Colorado.

Whelan, K.R.T. 2011. Schaus Swallowtail butterfly (Heraclides aristodemus ponceanus) habitat enhancement in Biscayne National Park. Project Report. Funded by FWS South Florida Coastal Program. National Park Service, Palmetto Bay, FL. 9 pp.

Williams, K., MacDonald, M., and Sternberg, L.D.S.L. 2003. Interactions of storm, drought, and sea-level rise on coastal forest: A case study. Journal of Coastal Research, 19(4): 1116-1121. 
TABLES

Table 2.1: Number and location of different plant species planted during the Schaus' Swallowtail Enhancement Project (Whelan and Atkinson, 2015). The majority of plants were sea torchwood, the primary host plant for the Schaus' swallowtail butterfly.

\begin{tabular}{|c|l|c|c|c|c|c|}
\hline \multirow{2}{*}{ Island } & Restoration Area & ha & $\begin{array}{c}\text { Torchwood } \\
\text { Planted }\end{array}$ & $\begin{array}{c}\text { Wild Lime } \\
\text { Planted }\end{array}$ & $\begin{array}{c}\text { Nectar Plants } \\
\text { Planted }\end{array}$ & $\begin{array}{c}\text { Total Plants } \\
\text { Planted to Date }\end{array}$ \\
\hline \multirow{5}{*}{ Elliott Key } & Elliott Main & 0.40 & 598 & 116 & 60 & 774 \\
\cline { 2 - 7 } & Elliott Breezeway & 0.18 & 433 & 102 & 2 & 537 \\
\cline { 2 - 7 } & $\begin{array}{l}\text { Elliott Spite Highway } \\
\text { North }\end{array}$ & N/A & 824 & - & - & 824 \\
\cline { 2 - 7 } & Elliott East & N/A & 58 & - & - & 58 \\
\cline { 2 - 7 } & Elliott West & N/A & 151 & - & - & 151 \\
\hline \multirow{2}{*}{ Adams Key } & Adams Main & 0.17 & 414 & 104 & 15 & 533 \\
\cline { 2 - 7 } & Adams Breezeway & 0.10 & 275 & 64 & 17 & 356 \\
\hline Totals & $\mathbf{2 . 1}$ & $\mathbf{2 , 7 5 3}$ & $\mathbf{3 8 6}$ & $\mathbf{9 4}$ & $\mathbf{3 , 2 3 3}$ \\
\hline
\end{tabular}


Table 2.2: Sea torchwood and wild lime survivorship at different restoration sites in BNP. The average amount of days ( \pm SE)

between measurements is listed in the table. $\mathrm{EKM}=$ Elliott Key Main Restoration Site, EKB = Elliott Key Breezeway Site, $\mathrm{AKM}$

= Adams Key Main Restoration Site, AKB = Adams Key Breezeway Site.

\begin{tabular}{|c|c|c|c|c|c|c|c|c|c|}
\hline \multicolumn{10}{|c|}{ Sea Torchwood } \\
\hline \multirow{2}{*}{ Sites } & \multicolumn{3}{|c|}{2013 (172.9 \pm 0.3 Days $)$} & \multicolumn{3}{|c|}{2014 (377.8 \pm 0.5 Days) } & \multicolumn{3}{|c|}{$2017(1,382.1 \pm 6.3$ Days $)$} \\
\hline & Alive & Dead & $\%$ Alive & Alive & Dead & $\%$ Alive & Alive & Dead & $\%$ Alive \\
\hline EKM & 385 & 212 & $64.5 \%$ & 385 & 212 & $64.5 \%$ & 347 & 250 & $58.1 \%$ \\
\hline EKB & 310 & 124 & $71.4 \%$ & 303 & 131 & $69.8 \%$ & 264 & 170 & $60.8 \%$ \\
\hline $\mathrm{AKM}$ & 236 & 180 & $56.7 \%$ & 223 & 193 & $53.6 \%$ & 187 & 229 & $45.0 \%$ \\
\hline $\mathrm{AKB}$ & 94 & 180 & $34.3 \%$ & 80 & 194 & $29.2 \%$ & 19 & 255 & $6.9 \%$ \\
\hline Overall & 1025 & 696 & $59.6 \%$ & 991 & 730 & $57.6 \%$ & 817 & 904 & $47.5 \%$ \\
\hline \multicolumn{10}{|c|}{ Wild Lime } \\
\hline \multirow{2}{*}{ Sites } & \multicolumn{4}{|c|}{2013 (166.3 \pm 0.6 Days $)$} & \multicolumn{5}{|c|}{2014 (373.9 \pm 0.7 Days) } \\
\hline & Alive & & Dead & $\%$ Alive & & live & Dead & & $\%$ Alive \\
\hline EKM & 114 & & 2 & $98.3 \%$ & & 114 & 2 & & $98.3 \%$ \\
\hline EKB & 91 & & 11 & $89.2 \%$ & & 89 & 13 & & $87.3 \%$ \\
\hline AKM & 97 & & 7 & $93.3 \%$ & & 96 & 8 & & $92.3 \%$ \\
\hline $\mathrm{AKB}$ & 56 & & 8 & $87.5 \%$ & & 54 & 10 & & $84.4 \%$ \\
\hline Overall & 358 & & 28 & $92.7 \%$ & & 353 & 33 & & $91.5 \%$ \\
\hline
\end{tabular}


Table 2.3: Results from the Kruskal-Wallis test including a post-hoc pairwise comparisons analysis between sites. Three independent variables $(\mathrm{CC}=$ Canopy Cover, Elev = Elevation, Soil Depth $)$ were measured for sea torchwood and one independent variable $($ Elev $=$ Elevation $)$ was measured for wild lime. $\mathrm{TS}=$ Test Statistic, $\mathrm{SE}=$ Standard Error, Sig $=$ Significance, $\mathrm{EKM}=$ Elliott Key Main Restoration Site, EKB = Elliott Key Breezeway Site, $\mathrm{AKM}=$ Adams Key Main Restoration Site, $\mathrm{AKB}=\mathrm{Adams}$ Key Breezeway Site.

\begin{tabular}{|c|c|c|c|c|c|c|c|c|c|}
\hline Sites & \multicolumn{2}{|c|}{ CC (percent overstory) } & \multicolumn{3}{c|}{ Elev (m) } & \multicolumn{3}{c|}{ Soil Depth (cm) } \\
\hline Torchwood & TS & SE & Sig & TS & SE & Sig & TS & SE & Sig \\
\hline EKM - EKB & 195.412 & 25.333 & $<0.001$ & 642.383 & 31.355 & $<0.001$ & 275.071 & 25.316 & $<0.001$ \\
\hline EKM - AKM & 221.286 & 28.425 & $<0.001$ & 820.984 & 31.746 & $<0.001$ & -227.545 & 28.308 & $<0.001$ \\
\hline EKM - AKB & 345.899 & 25.385 & $<0.001$ & $1,139.458$ & 36.274 & $<0.001$ & 168.994 & 25.316 & $<0.001$ \\
\hline EKB - AKM & 25.874 & 29.804 & 0.385 & 178.601 & 34.118 & $<0.001$ & -502.616 & 29.705 & $<0.001$ \\
\hline EKB - AKB & 150.487 & 26.921 & $<0.001$ & 497.075 & 38.368 & $<0.001$ & -106.077 & 26.869 & $<0.001$ \\
\hline AKM - AKB & 124.613 & 29.849 & $<0.001$ & 318.474 & 38.688 & $<0.001$ & 396.539 & 29.705 & $<0.001$ \\
\hline Wild Lime & TS & SE & Sig & TS & SE & Sig & TS & SE & Sig \\
\hline EKM - EKB & -- & -- & -- & 177.603 & 15.145 & $<0.001$ & -- & -- & -- \\
\hline EKM - AKM & -- & -- & -- & 170.742 & 15.067 & $<0.001$ & -- & -- & -- \\
\hline EKM - AKB & -- & -- & -- & 252.616 & 17.373 & $<0.001$ & -- & -- & -- \\
\hline EKB - AKM & -- & -- & -- & -6.861 & 15.548 & 0.659 & -- & -- & -- \\
\hline EKB - AKB & -- & -- & -- & 75.013 & 17.792 & $<0.001$ & -- & -- & -- \\
\hline AKM - AKB & -- & -- & -- & 81.874 & 17.726 & $<0.001$ & -- & -- & -- \\
\hline
\end{tabular}


Table 2.4: Independent variables $(\mathrm{CC}=$ Canopy Cover, Elev = Elevation, Soil Depth $)$ measured in the restored and breezeway sites at Elliott Key and Adams Key. Elevation was the only independent variable measured for wild lime. Median values were reported instead of average values because some data was not normally distributed for specific years and several sites. EKM = Elliott Key Main Restoration Site, EKB = Elliott Key Breezeway Site, AKM = Adams Key Main Restoration Site, AKB = Adams Key Breezeway Site.

\begin{tabular}{|c|l|c|c|c|c|c|c|c|c|c|}
\hline Species & Sites & \multicolumn{4}{c|}{ CC (percent overstory) } & \multicolumn{3}{c|}{ Elev (m) } & \multicolumn{3}{c|}{ Soil Depth (cm) } \\
\hline & & Median & \multicolumn{2}{|c|}{ Range } & Median & \multicolumn{2}{c|}{ Range } & Median & \multicolumn{2}{c|}{ Range } \\
\hline Torch & EKM & 0.5 & 0.0 & 1.0 & 2.8 & 0.0 & 3.5 & 13.0 & 4.7 & 26.9 \\
\hline Torch & EKB & 0.2 & 0.0 & 1.0 & 0.8 & 0.6 & 1.7 & 10.2 & 4.3 & 19.5 \\
\hline Torch & AKM & 0.2 & 0.0 & 1.0 & 0.7 & 0.5 & 1.3 & 18.2 & 6.3 & 37.80 \\
\hline Torch & AKB & 0.1 & 0.0 & 1.0 & 0.6 & 0.4 & 0.8 & 11.3 & 4.2 & 22.0 \\
\hline WL & EKM & -- & -- & -- & 2.9 & 1.6 & 3.5 & -- & -- & -- \\
\hline WL & EKB & -- & -- & -- & 0.7 & 0.6 & 1.7 & -- & -- & -- \\
\hline WL & AKM & -- & -- & -- & 0.8 & 0.2 & 1.2 & -- & -- & -- \\
\hline WL & AKB & -- & -- & -- & 0.6 & 0.3 & 0.9 & -- & -- & -- \\
\hline
\end{tabular}


Table 2.5: Results from the Kruskal-Wallis test including a post-hoc pairwise comparisons analysis between sites. Stem elongation (growth) was measured in 2013, 2014, and 2017 (see Table 2 for the number of growing days after the first measurement). Wild lime growth was measured in 2013 and 2014. TS = Test Statistic, SE = Standard Error, Sig = Significance, EKM = Elliott Key Main Restoration Site, EKB = Elliott Key Breezeway Site, AKM = Adams Key Main Restoration Site, AKB = Adams Key Breezeway Site.

\begin{tabular}{|c|c|c|c|c|c|c|c|c|c|}
\hline Sites & \multicolumn{3}{|c|}{$\mathbf{2 0 1 3}$} & \multicolumn{3}{c|}{$\mathbf{2 0 1 4}$} & \multicolumn{3}{c|}{$\mathbf{2 0 1 7}$} \\
\hline Torchwood & TS & SE & Sig & TS & SE & Sig & TS & SE & Sig \\
\hline EKM - EKB & 155.780 & 22.430 & $<0.001$ & 132.292 & 21.758 & $<0.001$ & 23.244 & 10.468 & 0.026 \\
\hline EKM - AKM & 419.822 & 24.456 & $<0.001$ & 391.479 & 23.819 & $<0.001$ & 74.220 & 11.134 & $<0.001$ \\
\hline EKM - AKB & 511.118 & 33.929 & $<0.001$ & 504.497 & 35.320 & $<0.001$ & 116.547 & 15.344 & $<0.001$ \\
\hline EKB - AKM & 264.043 & 25.504 & $<0.001$ & 259.187 & 24.980 & $<0.001$ & 50.975 & 12.452 & $<0.001$ \\
\hline EKB - AKB & 355.338 & 34.692 & $<0.001$ & 372.205 & 36.113 & $<0.001$ & 93.303 & 16.326 & $<0.001$ \\
\hline AKM - AKB & 91.295 & 36.035 & 0.011 & 113.018 & 37.391 & 0.003 & 42.328 & 16.760 & 0.012 \\
\hline Wild Lime & TS & SE & Sig & TS & SE & Sig & TS & SE & Sig \\
\hline EKM - EKB & 85.795 & 14.487 & $<0.001$ & 93.671 & 14.245 & $<0.001$ & -- & -- & -- \\
\hline EKM - AKM & 91.552 & 14.234 & $<0.001$ & 87.547 & 13.820 & $<0.001$ & -- & -- & -- \\
\hline EKM - AKB & 143.915 & 16.749 & $<0.001$ & 153.962 & 16.889 & $<0.001$ & -- & -- & -- \\
\hline EKB - AKM & 5.757 & 15.015 & 0.701 & -6.124 & 14.723 & 0.677 & -- & -- & -- \\
\hline EKB - AKB & 58.120 & 17.418 & 0.001 & 60.292 & 17.635 & 0.001 & -- & -- & -- \\
\hline AKM - AKB & 52.363 & 17.207 & 0.002 & 66.415 & 17.294 & $<0.001$ & -- & -- & -- \\
\hline
\end{tabular}


Table 2.6: Stem elongation (growth) of wild lime and sea torchwood in the restored and breezeway sites at Elliott Key and Adams Key. Median values were reported instead of average values because growth data was not normally distributed for specific years and at several sites. EKM = Elliott Key Main Restoration Site, EKB = Elliott Key Breezeway Site, $\mathrm{AKM}=$ Adams Key Main Restoration Site, $\mathrm{AKB}=$ Adams Key Breezeway Site.

\begin{tabular}{|c|c|c|c|c|c|c|}
\hline Species & Year & Sites & N & Median & Min & Max \\
\hline Torch & 2013 & EKM & 382 & 3.874 & -2.326 & 12.911 \\
\hline Torch & 2013 & EKB & 310 & 2.241 & -2.255 & 11.578 \\
\hline Torch & 2013 & AKM & 231 & 0.437 & -1.372 & 4.391 \\
\hline Torch & 2013 & AKB & 93 & 0.228 & -0.935 & 1.492 \\
\hline Torch & 2014 & EKM & 379 & 3.269 & -0.956 & 9.769 \\
\hline Torch & 2014 & EKB & 298 & 2.094 & -1.062 & 10.846 \\
\hline Torch & 2014 & AKM & 220 & 0.644 & -0.961 & 3.327 \\
\hline Torch & 2014 & AKB & 76 & 0.272 & -0.359 & 1.809 \\
\hline Torch & 2017 & EKM & 95 & 3.083 & 0.293 & 8.117 \\
\hline Torch & 2017 & EKB & 53 & 2.325 & -0.030 & 7.708 \\
\hline Torch & 2017 & AKM & 44 & 1.240 & -0.052 & 3.539 \\
\hline Torch & 2017 & AKB & 19 & 0.444 & 0.008 & 1.690 \\
\hline WL & 2013 & EKM & 111 & 1.074 & -0.923 & 6.162 \\
\hline WL & 2013 & EKB & 83 & 0.332 & -0.629 & 1.513 \\
\hline WL & 2013 & AKM & 95 & 0.296 & -1.900 & 2.714 \\
\hline WL & 2013 & AKB & 50 & -0.149 & -1.664 & 1.491 \\
\hline WL & 2014 & EKM & 111 & 1.076 & -0.764 & 4.854 \\
\hline WL & 2014 & EKB & 83 & 0.406 & -0.296 & 1.842 \\
\hline WL & 2014 & AKM & 95 & 0.355 & -0.900 & 2.226 \\
\hline WL & 2014 & AKB & 50 & -0.009 & -0.659 & 1.904 \\
\hline
\end{tabular}


Table 2.7: Results from the stepwise linear regression analysis. Best fit models were assembled with the most relevant independent variables. Torch $=$ Sea Torchwood, WL $=$ Wild Lime, Elev $=$ Ground elevation, Soil $=$ Relative soil depth, CC $=$ Canopy Cover .

\begin{tabular}{|c|c|c|c|c|c|c|c|c|c|c|c|c|c|c|c|}
\hline \multicolumn{16}{|c|}{ Linear Regression Model Summary } \\
\hline Species & $\mathrm{N}$ & & Year & \multicolumn{2}{|c|}{ Variable(s) } & \multicolumn{2}{|l|}{$\mathrm{R}$} & \multicolumn{2}{|c|}{$\mathrm{R}^{2}$} & Adj. $\mathrm{R}^{2}$ & \multicolumn{4}{|c|}{ Std. Err } & \\
\hline Torch & 663 & & 2013 & \multicolumn{2}{|c|}{$\beta_{1}$-Elev } & \multicolumn{2}{|c|}{0.545} & 0.297 & \multicolumn{2}{|c|}{0.296} & \multicolumn{4}{|c|}{2.130} & \\
\hline Torch & 628 & & 2014 & \multicolumn{2}{|c|}{$\beta_{1}$-Elev/ $\beta_{2}-\mathrm{CC}$} & \multicolumn{2}{|c|}{0.532} & 0.283 & \multicolumn{2}{|c|}{0.281} & \multicolumn{4}{|c|}{1.777} & \\
\hline Torch & 191 & & 2017 & \multicolumn{2}{|c|}{$\beta_{1}$-Elev $/ \beta_{2}$-Soil } & \multicolumn{2}{|l|}{0.506} & 0.256 & \multicolumn{2}{|c|}{0.248} & \multicolumn{4}{|c|}{1.469} & \\
\hline WL & 354 & & 2013 & \multicolumn{2}{|c|}{$\beta_{1}$-Elev } & \multicolumn{2}{|l|}{0.505} & 0.255 & \multicolumn{2}{|c|}{0.253} & \multicolumn{4}{|c|}{0.919} & \\
\hline WL & 343 & & 2014 & & Elev & 0.530 & & 0.28 & & & & & 0.723 & & \\
\hline \multicolumn{7}{|c|}{ Anova (Regression Model) } & \multicolumn{9}{|c|}{ Standardized Coefficients } \\
\hline \multicolumn{2}{|c|}{ Species } & $\mathrm{df}$ & \multicolumn{2}{|c|}{ Mean $\mathrm{Sq}$} & $\mathrm{F}$ & Sig & \multicolumn{3}{|c|}{ Beta } & \multicolumn{3}{|c|}{$\mathrm{t}$} & \multicolumn{3}{|c|}{ Sig } \\
\hline & & & & & & & $\beta_{0}$ & $\beta_{1}$ & $\beta_{2}$ & $\beta_{0}$ & $\beta_{1}$ & $\beta_{2}$ & $\beta_{0}$ & $\beta_{1}$ & $\beta_{2}$ \\
\hline \multicolumn{2}{|c|}{ Torch (13) } & 1 & & 5.826 & 283.531 & $<0.001$ & -- & 0.545 & -- & 2.89 & 16.838 & -- & 0.004 & $<0.001$ & -- \\
\hline Torch ( & & 2 & & .983 & 126.369 & $<0.001$ & -- & 0.563 & -0.207 & 8.08 & 15.886 & -5.824 & $<0.001$ & $<0.001$ & $<0.001$ \\
\hline Torch ( & & 2 & & 714 & 33.228 & $<0.001$ & -- & 0.492 & -0.176 & 5.85 & 7.882 & -2.816 & $<0.001$ & $<0.001$ & 0.005 \\
\hline WL (1 & & 1 & & .750 & 120.513 & $<0.001$ & -- & 0.505 & -- & -1.94 & 10.978 & -- & 0.052 & $<0.001$ & -- \\
\hline WL (1 & & 1 & & 599 & 133.097 & $<0.001$ & -- & 0.530 & -- & 0.56 & 11.537 & -- & 0.575 & $<0.001$ & -- \\
\hline
\end{tabular}


Table 2.8: Results from the binary logistic regression analysis. Best fit models were assembled with the most relevant independent variables. C/S r ${ }^{2}=$ Cox and Snell $r^{2}$, Nag $r^{2}=$ Nagelkerke $r^{2}, \mathrm{H} \&$ L test $=$ Hosmer-Lemeshow test, $\mathrm{T}=$ Sea torchwood, WL $=$ Wild Lime, Elev = Ground elevation, Soil = Relative soil depth, $\mathrm{CC}=$ Canopy Cover.

\begin{tabular}{|c|c|c|c|c|c|c|c|c|c|c|c|c|}
\hline \multirow{2}{*}{ Models } & \multirow{2}{*}{$\mathrm{N}$} & \multicolumn{2}{|c|}{ Pseudo $r^{2}$} & \multicolumn{3}{|c|}{ Variables } & \multicolumn{4}{|c|}{ Coefficients } & \multicolumn{2}{|c|}{ H \& L test } \\
\hline & & $\mathrm{C} / \mathrm{S} \mathrm{r}^{2}$ & $\operatorname{Nag} r^{2}$ & $X_{1}$ & $\mathrm{X}_{2}$ & $\mathrm{X}_{3}$ & $\beta_{0}$ & $\beta_{1}$ & $\beta_{2}$ & $\beta_{3}$ & $\chi^{2}$ & $P$ \\
\hline $\mathrm{T}(2013)$ & 1073 & 0.064 & 0.087 & Elev & Soil & -- & -1.037 & 0.398 & 0.076 & -- & 11.168 & 0.192 \\
\hline $\mathrm{T}(2014)$ & 1073 & 0.081 & 0.109 & Elev & Soil & -- & -1.284 & 0.481 & 0.082 & -- & 14.349 & 0.073 \\
\hline $\mathrm{T}(2017)$ & 1073 & 0.114 & 0.153 & Elev & Soil & $\mathrm{CC}$ & -2.124 & 0.514 & 0.062 & 0.972 & 15.338 & 0.053 \\
\hline WL (2013) & 386 & 0.034 & 0.084 & Elev & -- & -- & 1.390 & 1.121 & -- & -- & 23.203 & 0.003 \\
\hline WL (2014) & 386 & 0.041 & 0.093 & Elev & -- & -- & 1.182 & 1.151 & -- & -- & 22.540 & 0.004 \\
\hline
\end{tabular}


Table 2.9: Receiver operating characteristic (ROC) curve results for sea torchwood and wild lime survivorship against increasing elevation and soil depth and decreasing canopy cover in restored and breezeway sites at Elliott Key and Adams Key. Accuracy of the ROC curve is measured by the area under the curve (AUC; $\mathrm{SE}=$ Standard Error). The AUC grade system: $>0.90-1.00=\operatorname{excellent}(\mathrm{A}),>0.80-0.90=\operatorname{good}(\mathrm{B}),>0.70-0.80$ $=$ fair $(C),>0.60-0.70=$ poor $(D), 0.50-0.60=$ fail $(F)$.

\begin{tabular}{|c|c|c|c|c|c|c|}
\hline \multicolumn{7}{|c|}{ Sea torchwood survivorship } \\
\hline \multirow{2}{*}{ Year } & Canopy Cover & Elevation & Soil Depth \\
\cline { 2 - 7 } & AUC & SE & AUC & SE & AUC & SE \\
\hline 2013 & 0.630 & 0.018 & 0.678 & 0.018 & 0.611 & 0.018 \\
\hline 2014 & 0.645 & 0.017 & 0.695 & 0.017 & 0.619 & 0.017 \\
\hline 2016 & 0.649 & 0.017 & 0.716 & 0.015 & 0.608 & 0.017 \\
\hline \multicolumn{7}{|c|}{ Wild lime survivorship } \\
\hline \multicolumn{7}{|c|}{ Elevation } \\
\hline AUC & 0.750 & \multicolumn{3}{c|}{0.049} \\
\hline Year & \multicolumn{7}{|c|}{0.738} & \multicolumn{3}{c|}{0.050} \\
\hline 2013 & \multicolumn{7}{|c|}{} \\
\hline 2014 & \multicolumn{7}{|c|}{0.04} \\
\hline
\end{tabular}


Table 2.10: Projected subtropical dry forest habitat loss in Biscayne National Park (BNP) and Key Largo from imminent sea level rise (SLR). Dry forest habitat initially starts $0.30 \mathrm{~m}$ above sea level (95\% of dry forest) in BNP and Key Largo. Remaining dry forest habitat (in hectares) at each site is presented in SLR increments of $25 \mathrm{~cm}$ up to $200 \mathrm{~cm}$. SK = Sands Key, EK= Elliott Key, AK = Adams Key, ORK = Old Rhodes Key, TK = Totten Key, PKCR = Porgy Key and Caesar Rock, SK = Swan Key, BNP = Biscayne National Park, PABLK = Palo Alto Key, Broad Key, and Linderman Key, CLNWR = Crocodile Lake National Wildlife Refuge, KLHSBP = Key Largo Hammock State Botanical Site, UNP = Unprotected Dry Forest Habitat, KLSDF = Key Largo South Dry Forest, KLTF = Key Largo Total Forest.

\begin{tabular}{|c|c|c|c|c|c|c|c|c|c|}
\hline Site & No SLR & $25 \mathrm{~cm}$ & $50 \mathrm{~cm}$ & $75 \mathrm{~cm}$ & $100 \mathrm{~cm}$ & $125 \mathrm{~cm}$ & $150 \mathrm{~cm}$ & $175 \mathrm{~cm}$ & $200 \mathrm{~cm}$ \\
\hline SK & 34.61 & $26.71(77 \%)$ & $18.79(54 \%)$ & $11.05(32 \%)$ & $3.82(11 \%)$ & $0.87(2.5 \%)$ & $0.05(0.1 \%)$ & $0.03(<0.1 \%)$ & $0.03(<0.1 \%)$ \\
\hline EK & 426.83 & $354.49(83 \%)$ & $267.37(63 \%)$ & $189.21(44 \%)$ & $121.71(29 \%)$ & $60.60(14 \%)$ & $18.63(4 \%)$ & $4.86(1 \%)$ & $2.17(0.5 \%)$ \\
\hline $\mathrm{AK}$ & 12.60 & $8.80(70 \%)$ & $3.41(27 \%)$ & $0.49(4 \%)$ & $0.01(<0.1 \%)$ & $0.00(0 \%)$ & $0.00(0 \%)$ & $0.00(0 \%)$ & $0.00(0 \%)$ \\
\hline ORK & 122.38 & $106.24(87 \%)$ & $84.20(69 \%)$ & $61.91(51 \%)$ & $43.40(36 \%)$ & $30.91(25 \%)$ & $17.47(14 \%)$ & $7.41(6 \%)$ & $1.92(2 \%)$ \\
\hline TK & 55.21 & $51.92(94 \%)$ & $47.75(87 \%)$ & $42.57(77 \%)$ & $36.53(66 \%)$ & $28.29(51 \%)$ & $16.79(30 \%)$ & $7.27(13 \%)$ & $2.85(5 \%)$ \\
\hline PKCR & 7.41 & $5.75(78 \%)$ & $4.56(62 \%)$ & $3.51(47 \%)$ & $2.42(33 \%)$ & $1.35(18 \%)$ & $0.40(5 \%)$ & $0.03(0.4 \%)$ & $0.00(0 \%)$ \\
\hline SK & 12.73 & $11.56(91 \%)$ & $9.35(73 \%)$ & $7.19(57 \%)$ & $4.72(37 \%)$ & $3.20(25 \%)$ & $2.30(18 \%)$ & $1.42(11 \%)$ & $0.50(4 \%)$ \\
\hline BNP & 671.77 & $565.47(84 \%)$ & $435.43(65 \%)$ & $315.93(47 \%)$ & $212.61(32 \%)$ & $125.22(19 \%)$ & $55.64(8 \%)$ & $21.02(3 \%)$ & $7.47(1 \%)$ \\
\hline PABLK & 31.23 & $29.82(96 \%)$ & $27.62(88 \%)$ & $25.25(81 \%)$ & $21.65(69 \%)$ & $16.43(53 \%)$ & $11.11(36 \%)$ & $4.00(13 \%)$ & $0.87(3 \%)$ \\
\hline CLNWR & 250.32 & $244.47(98 \%)$ & $237.06(95 \%)$ & $228.62(91 \%)$ & $218.47(87 \%)$ & $204.89(82 \%)$ & $187.21(75 \%)$ & $165.92(66 \%)$ & $135.76(54 \%)$ \\
\hline KLHBSP & 542.98 & $497.65(92 \%)$ & $431.17(79 \%)$ & $363.26(67 \%)$ & $286.26(53 \%)$ & $207.05(38 \%)$ & $151.25(28 \%)$ & $106.49(20 \%)$ & $66.26(12 \%)$ \\
\hline UNP & 150.71 & $139.53(93 \%)$ & $124.87(83 \%)$ & $110.97(74 \%)$ & $99.68(66 \%)$ & $87.33(58 \%)$ & $74.07(49 \%)$ & $60.19(40 \%)$ & $47.29(31 \%)$ \\
\hline KLSDF & 488.34 & $445.95(91 \%)$ & $400.95(82 \%)$ & $359.10(74 \%)$ & $319.33(65 \%)$ & $276.25(57 \%)$ & $241.13(49 \%)$ & $210.37(43 \%)$ & $175.77(36 \%)$ \\
\hline KLTF & 1463.58 & $1357.42(93 \%)$ & $1221.67(84 \%)$ & $1087.2(74 \%)$ & $945.39(65 \%)$ & $791.95(54 \%)$ & $664.77(45 \%)$ & $546.97(37 \%)$ & $425.95(29 \%)$ \\
\hline TOTAL & 2135.35 & $1922.89(90 \%)$ & $1657.10(78 \%)$ & $1403.13(66 \%)$ & $1158.00(54 \%)$ & $917.17(43 \%)$ & $720.41(34 \%)$ & $567.99(27 \%)$ & $433.42(20 \%)$ \\
\hline
\end{tabular}




\section{FIGURES}

Figure 2.1: Schaus' swallowtail early instar caterpillar resting on sea torchwood in a subtropical dry forest habitat at Elliott Key in Biscayne National Park, Florida. Sea torchwood is the primary host plant for Schaus' swallowtail caterpillars (Minno and Emmel, 1993; Jameson, 2002).

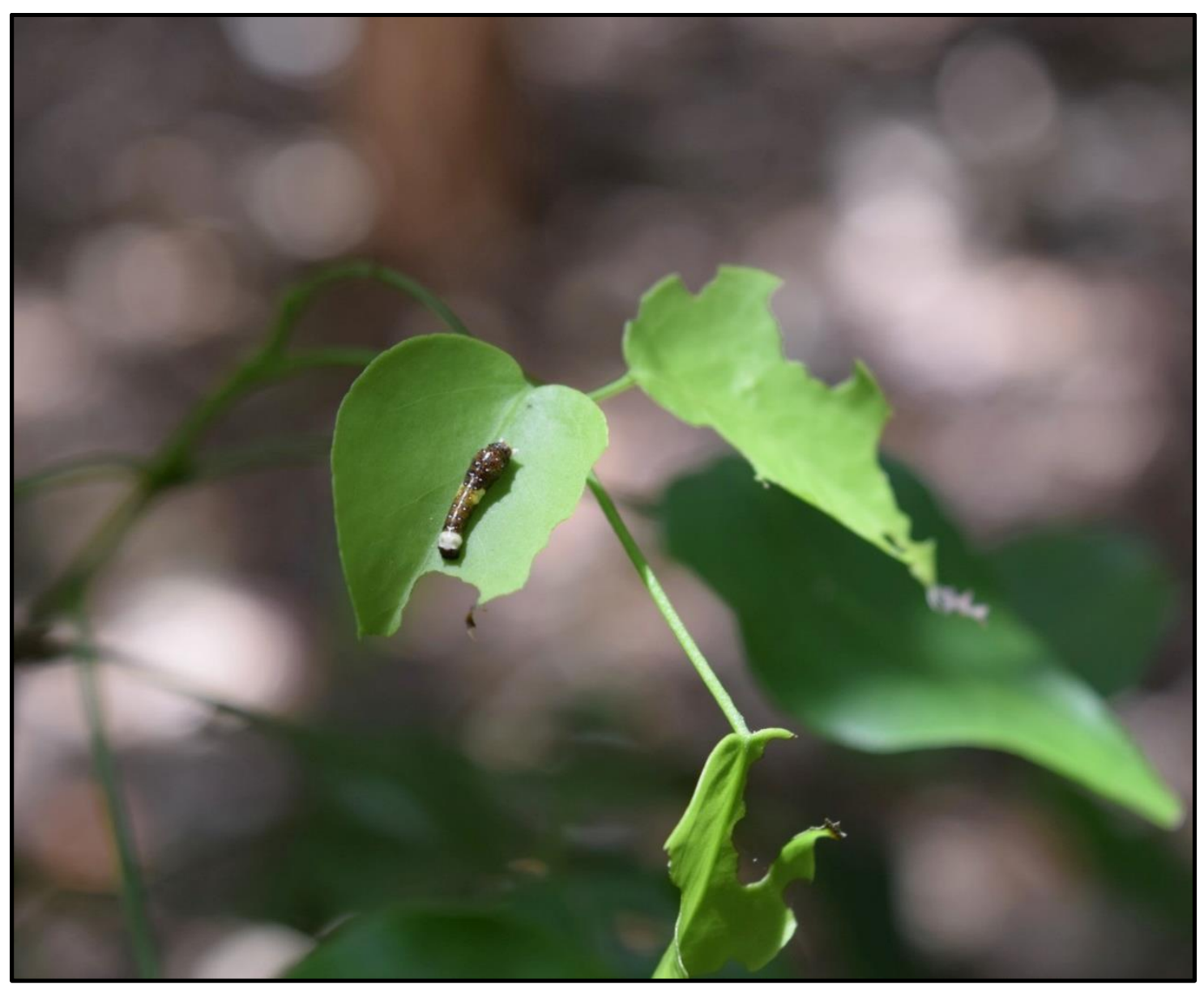


Figure 2.2: The Keys that incorporate terrestrial landmass within the boundaries of Biscayne National Park (BNP) and North Key Largo in south Florida. The light-green color displays the extent of subtropical dry forest in BNP and North Key Largo. Sea torchwood, the primary host plant for the Schaus' swallowtail butterfly, predominantly grows in dry forest habitats (Lugo et al., 1978; Stalter et al., 1999; Jameson, 2002).

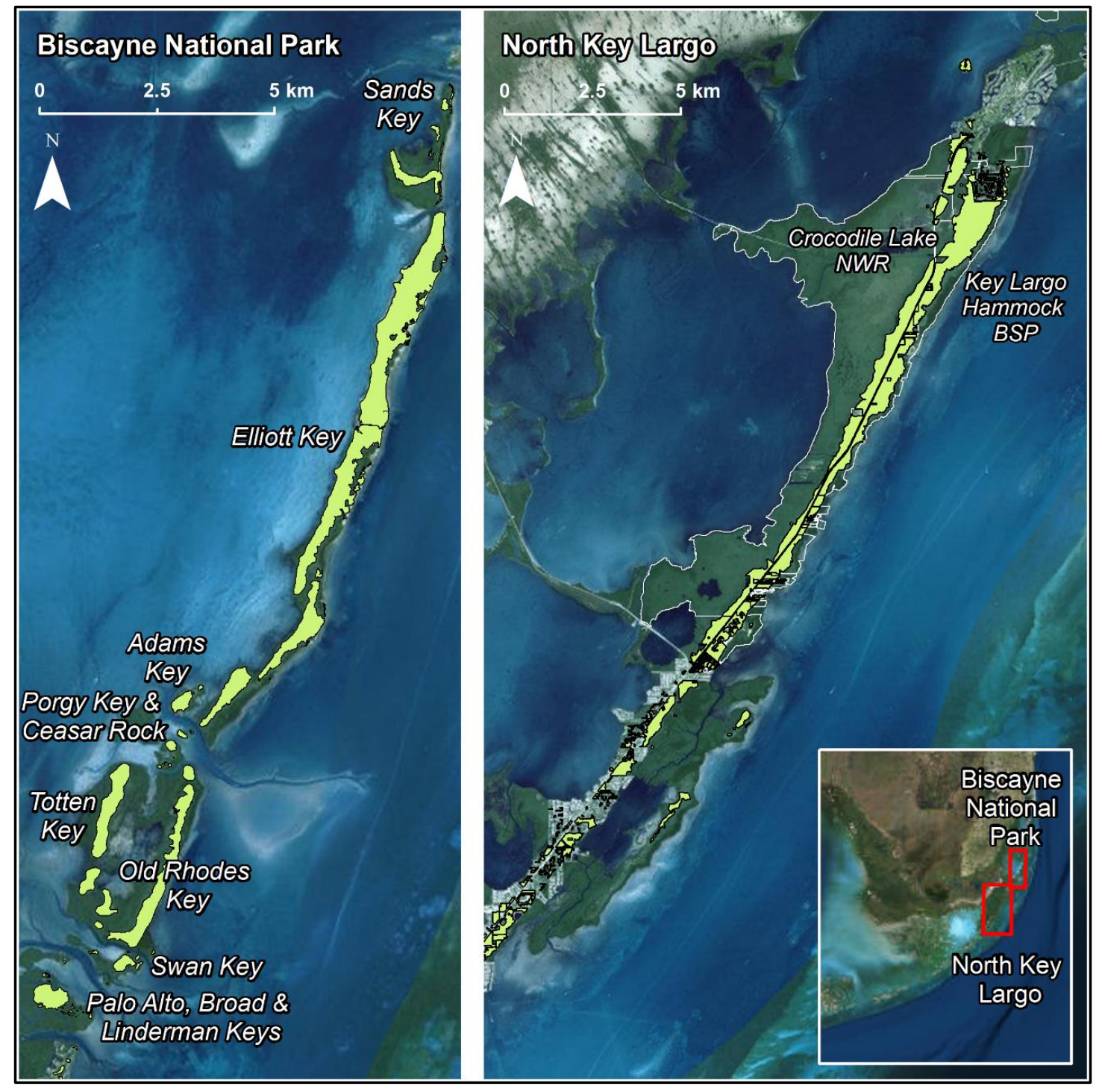


Figure 2.3: Restoration site locations, indicated with red boxes, of the Schaus'

Swallowtail Habitat Enhancement Project in Biscayne National Park (BNP), Florida (left). The red lines outline the restoration sites at Elliott Key (top-right) and Adams Key (bottom-right) in BNP (Whelan, 2011).

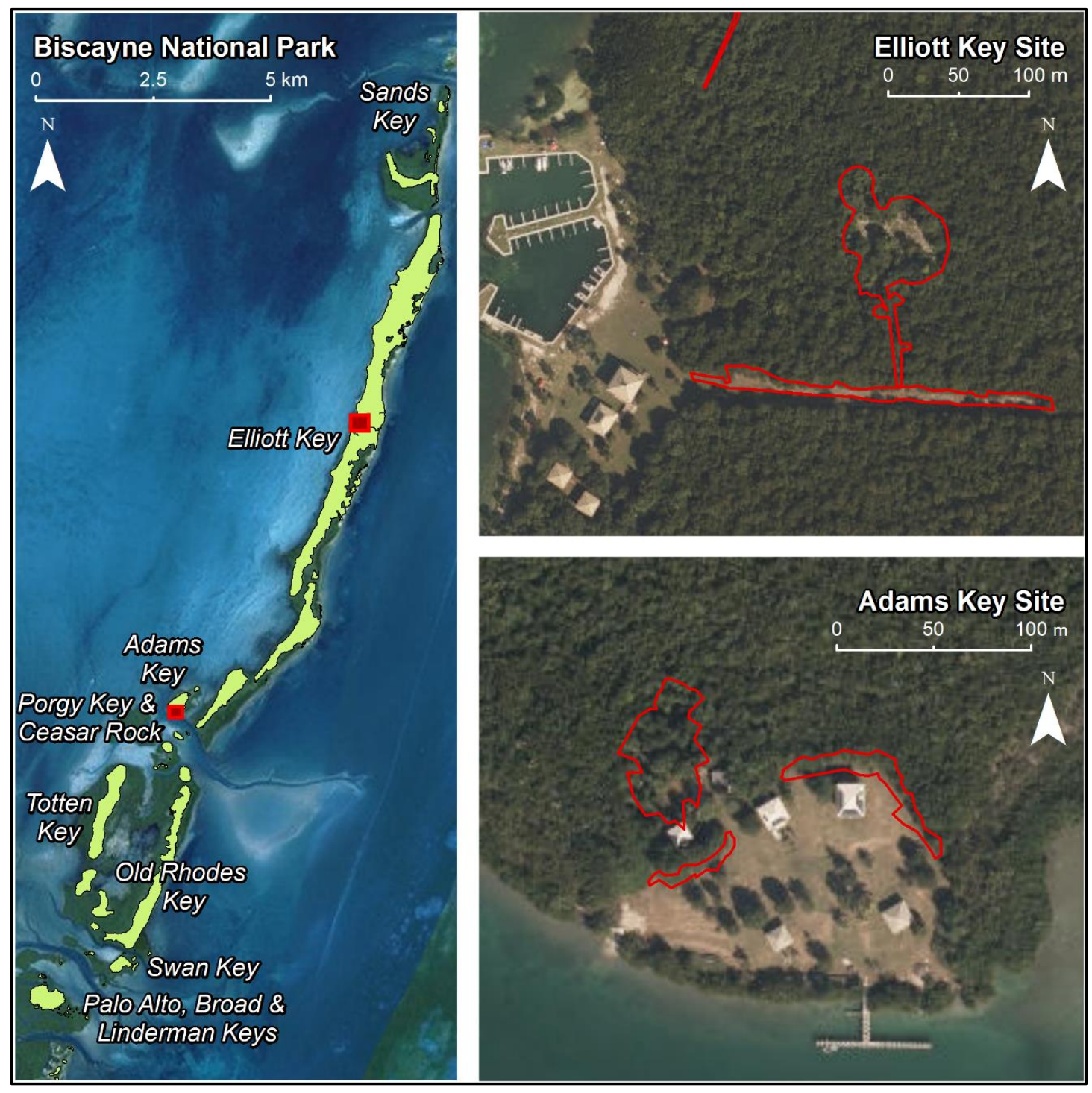


Figure 2.4: Sea torchwood planted in the restoration site at Elliott Key in Biscayne

National Park, Florida. Each plant received an aluminum tag to track survivorship and

primary stem elongation. They also received mulch to minimize water loss from substrate

desiccation. Leaf litter acts as a natural mulch in subtropical dry forest habitats (Ross et

al., 2003).

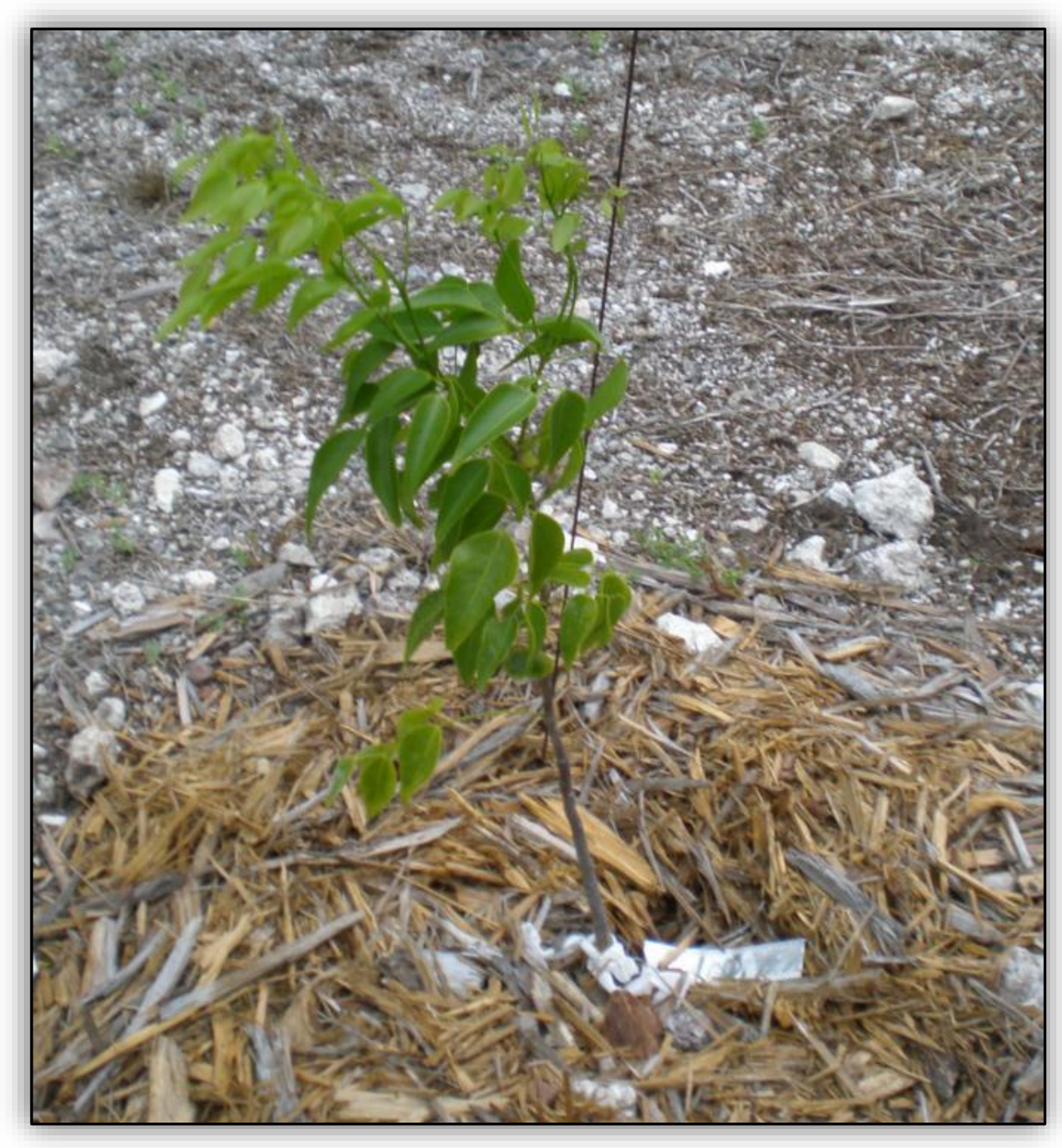


Figure 2.5: Elliott Key is a low-lying island within the boundaries of Biscayne National Park (BNP). Naturally occurring sea torchwood thrives at higher elevations within subtropical dry forest habitat at Elliott Key $(n=54, \overline{\mathrm{x}}=1.10 \mathrm{~m}, \mathrm{~s}=0.28)$.

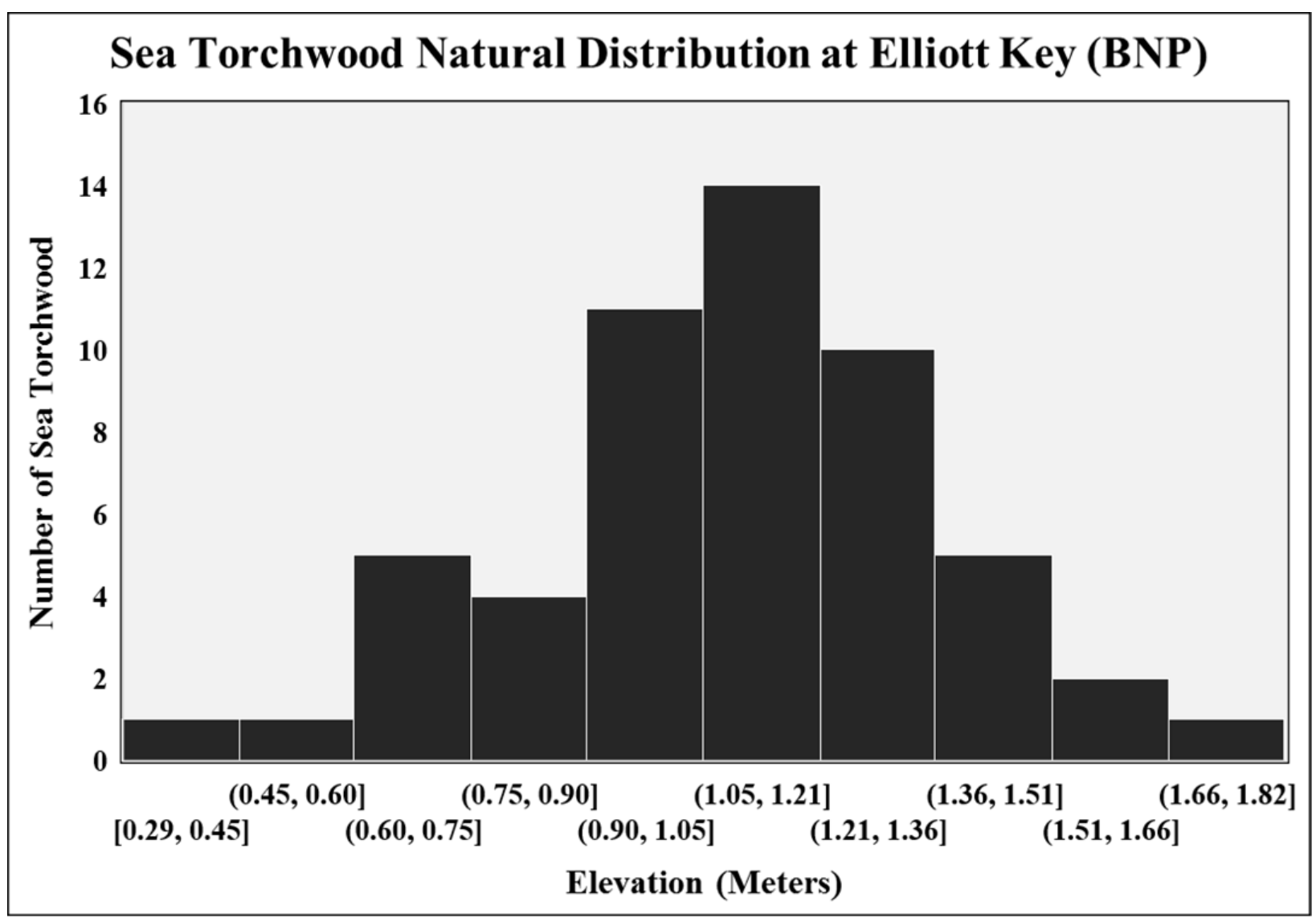


Figure 2.6: Boxplots displaying canopy cover (\% overstory) at each site. Canopy cover was recorded above each sea torchwood planted in the restored and breezeway sites at Elliott Key and Adams Key. Different letters above boxplots denote significance. Open circles represent suspected outliers; however, extreme outliers were removed. EK Main = Elliott Key Main Restoration Site, EK Breeze $=$ Elliott Key Breezeway Site, AK Main = Adams Key Main Restoration Site, AK Breeze = Adams Key Breezeway Site.

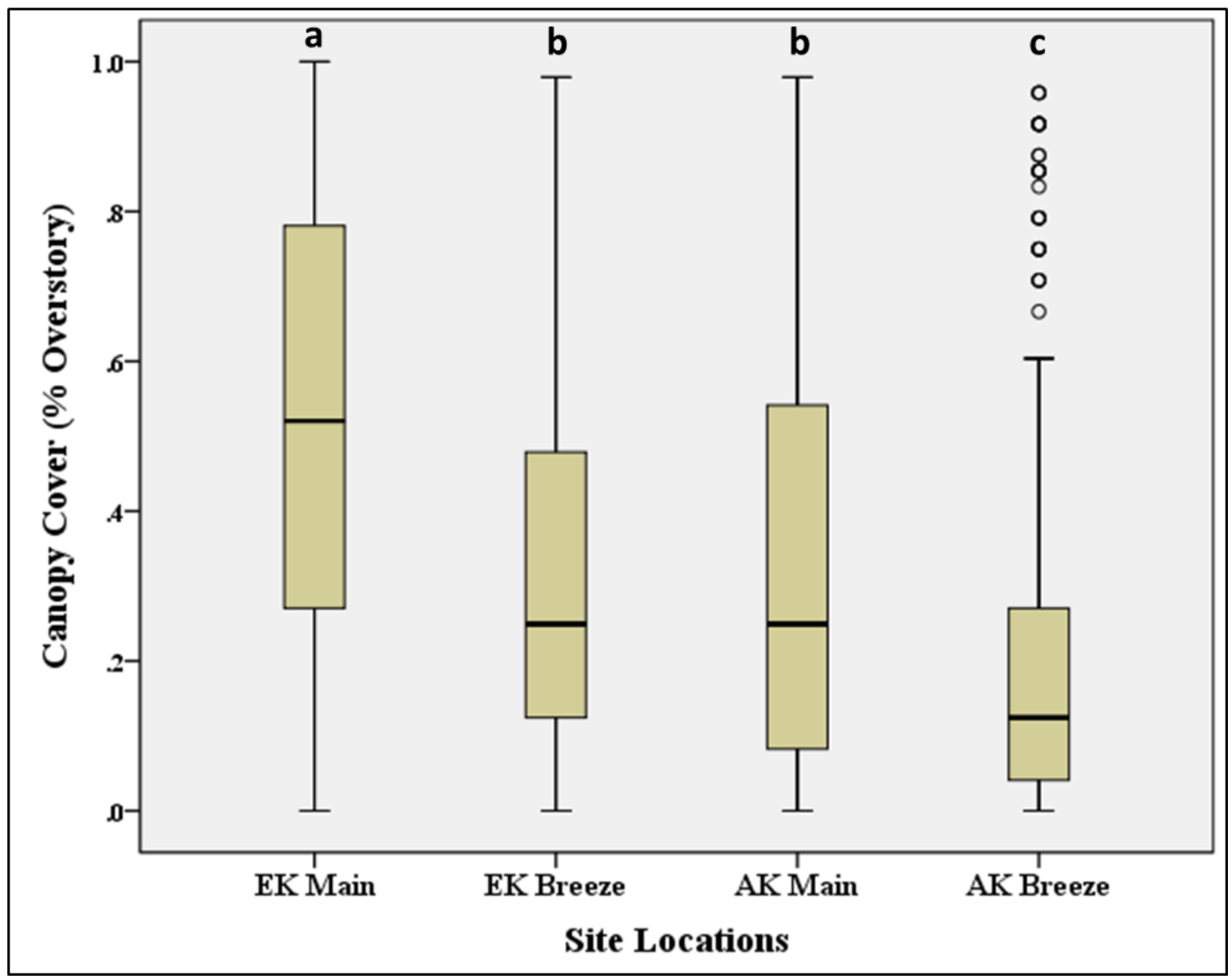


Figure 2.7: Boxplots displaying land elevation (m) at each site. Land elevation was recorded for each sea torchwood planted in the restored and breezeway sites. Elevation at each site was significantly different from other. Different letters above boxplots denote significance. Open circles represent suspected outliers; however, extreme outliers were removed. EK Main = Elliott Key Main Restoration Site, EK Breeze = Elliott Key Breezeway Site, AK Main = Adams Key Main Restoration Site, AK Breeze $=$ Adams Key Breezeway Site.

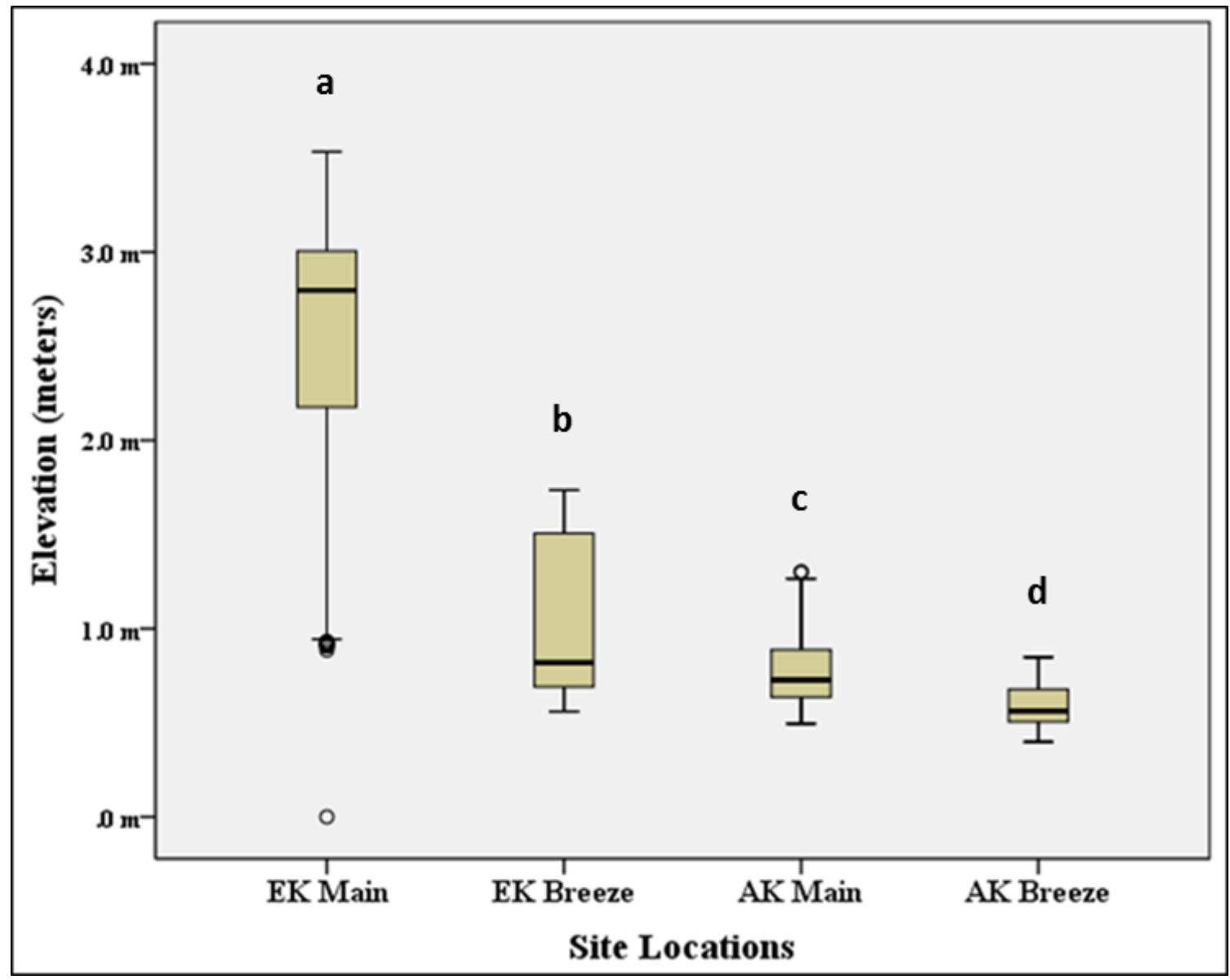


Figure 2.8: Boxplots displaying soil depth $(\mathrm{cm})$ at each site. Soil depth was recorded at each sea torchwood planted in the restored and breezeway sites. Different letters above boxplots denote significance. Open circles represent suspected outliers; however, extreme outliers were removed. EK Main = Elliott Key Main Restoration Site, EK Breeze = Elliott Key Breezeway Site, AK Main = Adams Key Main Restoration Site, AK Breeze = Adams Key Breezeway Site.

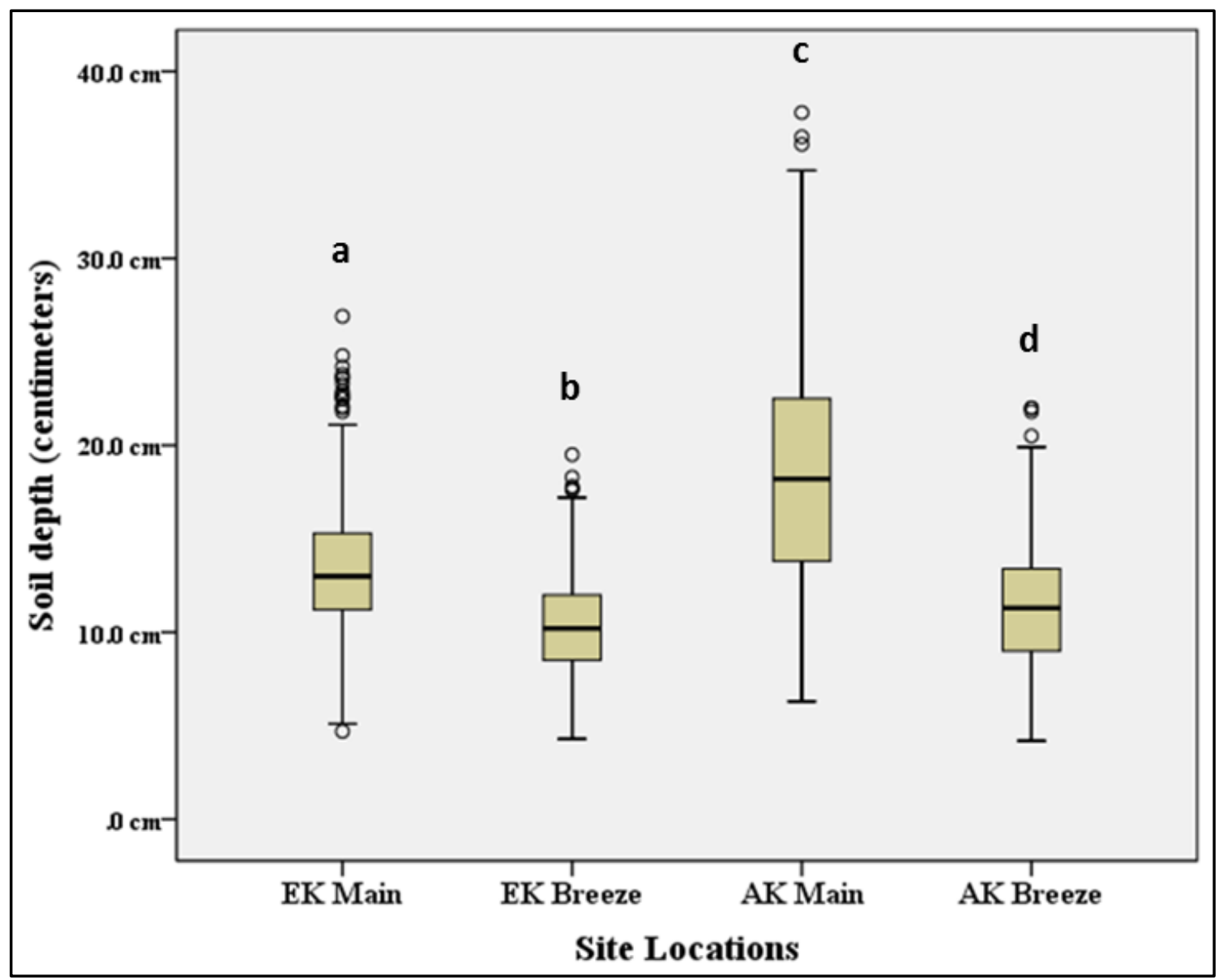


Figure 2.9: Box plots displaying sea torchwood stem elongation (growth in $\mathrm{cm}$ ) at each site during 2013. Different letters above boxplots denote significance. Open circles represent suspected outliers; however, extreme outliers were removed. EK Main = Elliott Key Main Restoration Site, EK Breeze = Elliott Key Breezeway Site, AK Main = Adams Key Main Restoration Site, AK Breeze = Adams Key Breezeway Site.

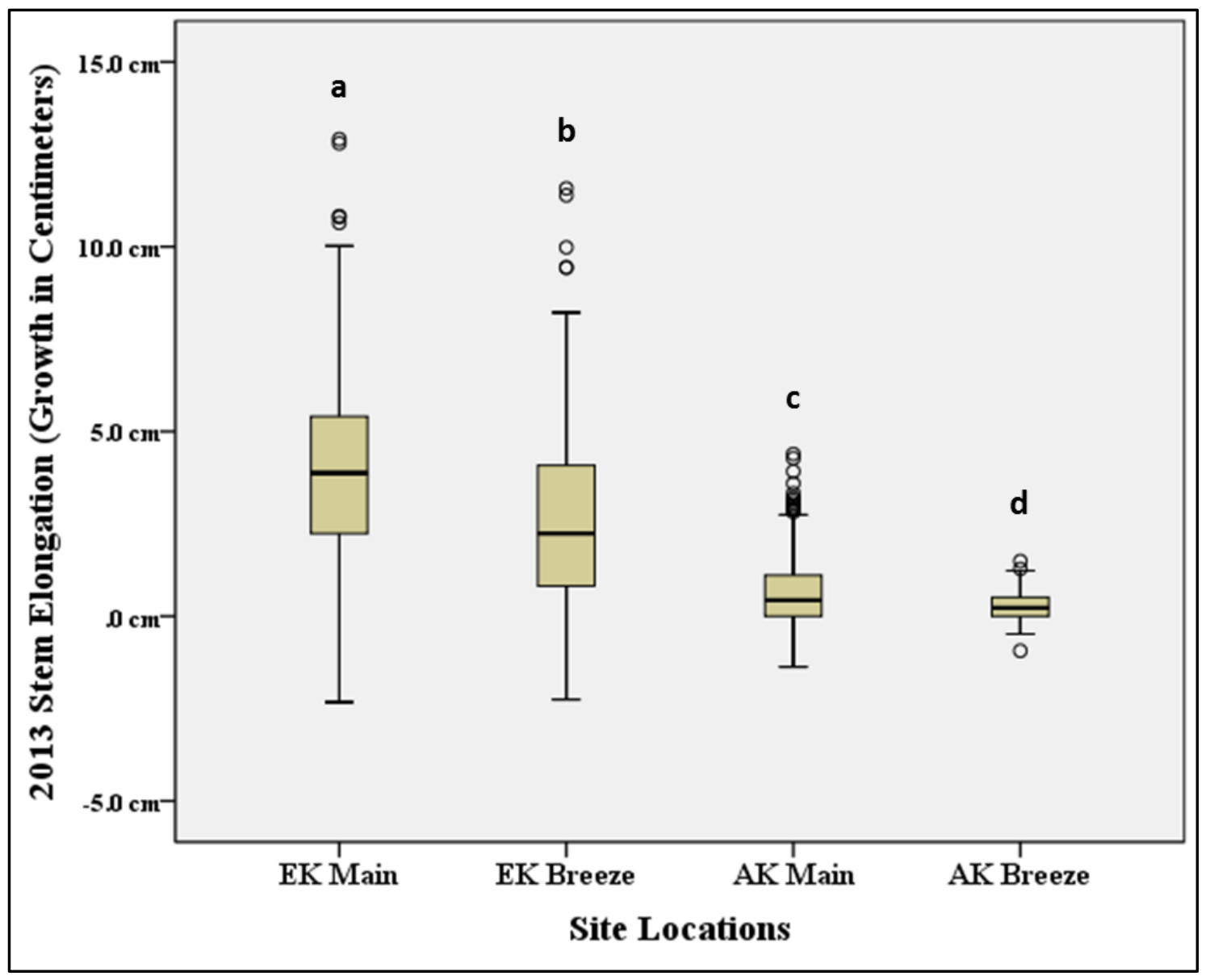


Figure 2.10: Box plots displaying sea torchwood stem elongation (growth in $\mathrm{cm}$ ) at each site during 2014. Different letters above boxplots denote significance. Open circles represent suspected outliers; however, extreme outliers were removed. EK Main = Elliott Key Main Restoration Site, EK Breeze = Elliott Key Breezeway Site, AK Main = Adams Key Main Restoration Site, AK Breeze = Adams Key Breezeway Site.

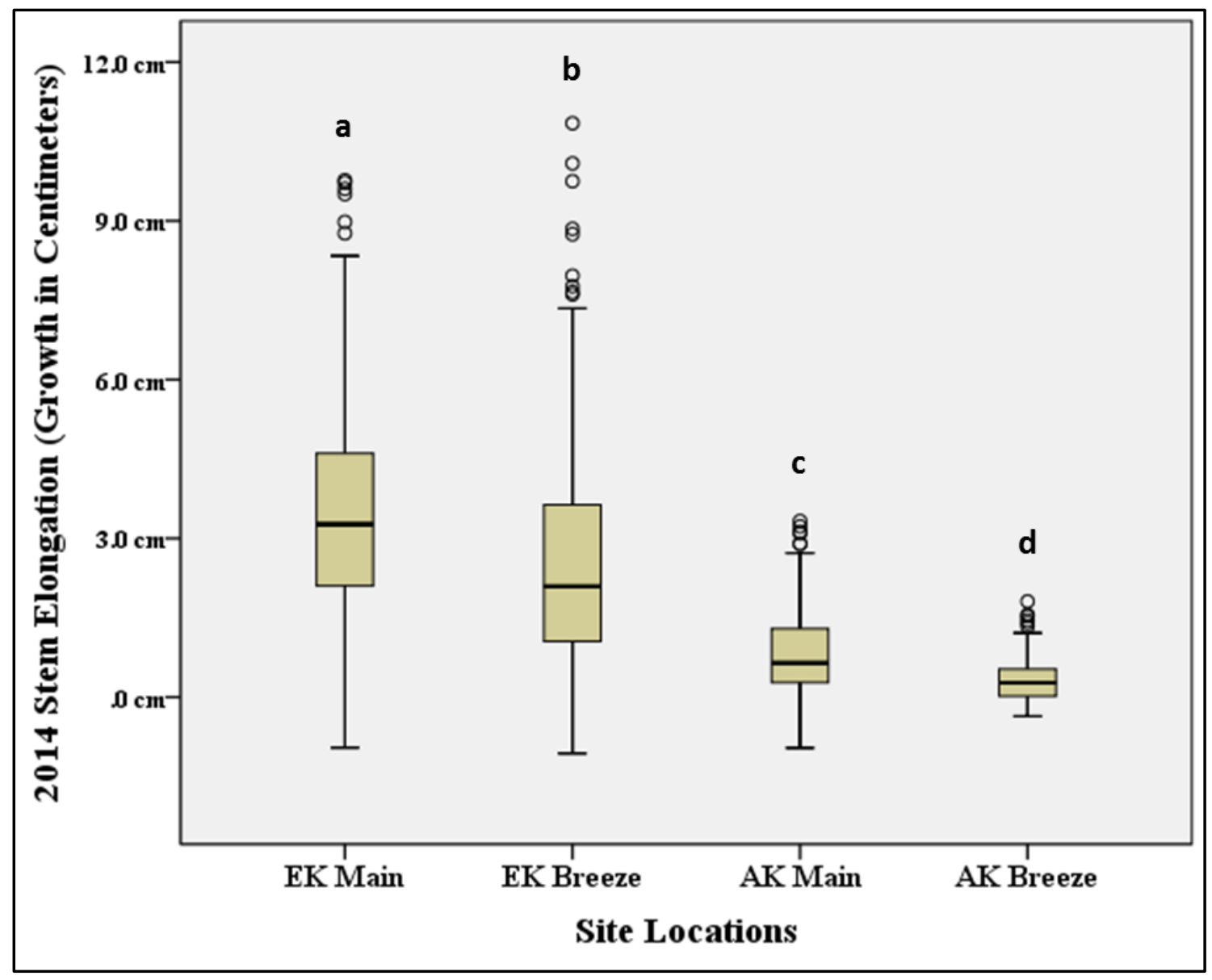


Figure 2.11: Box plots displaying sea torchwood stem elongation (growth in $\mathrm{cm}$ ) at each site during 2017. Different letters above boxplots denote significance. Open circles represent suspected outliers; however, extreme outliers were removed. EK Main = Elliott Key Main Restoration Site, EK Breeze = Elliott Key Breezeway Site, AK Main = Adams Key Main Restoration Site, AK Breeze = Adams Key Breezeway Site.

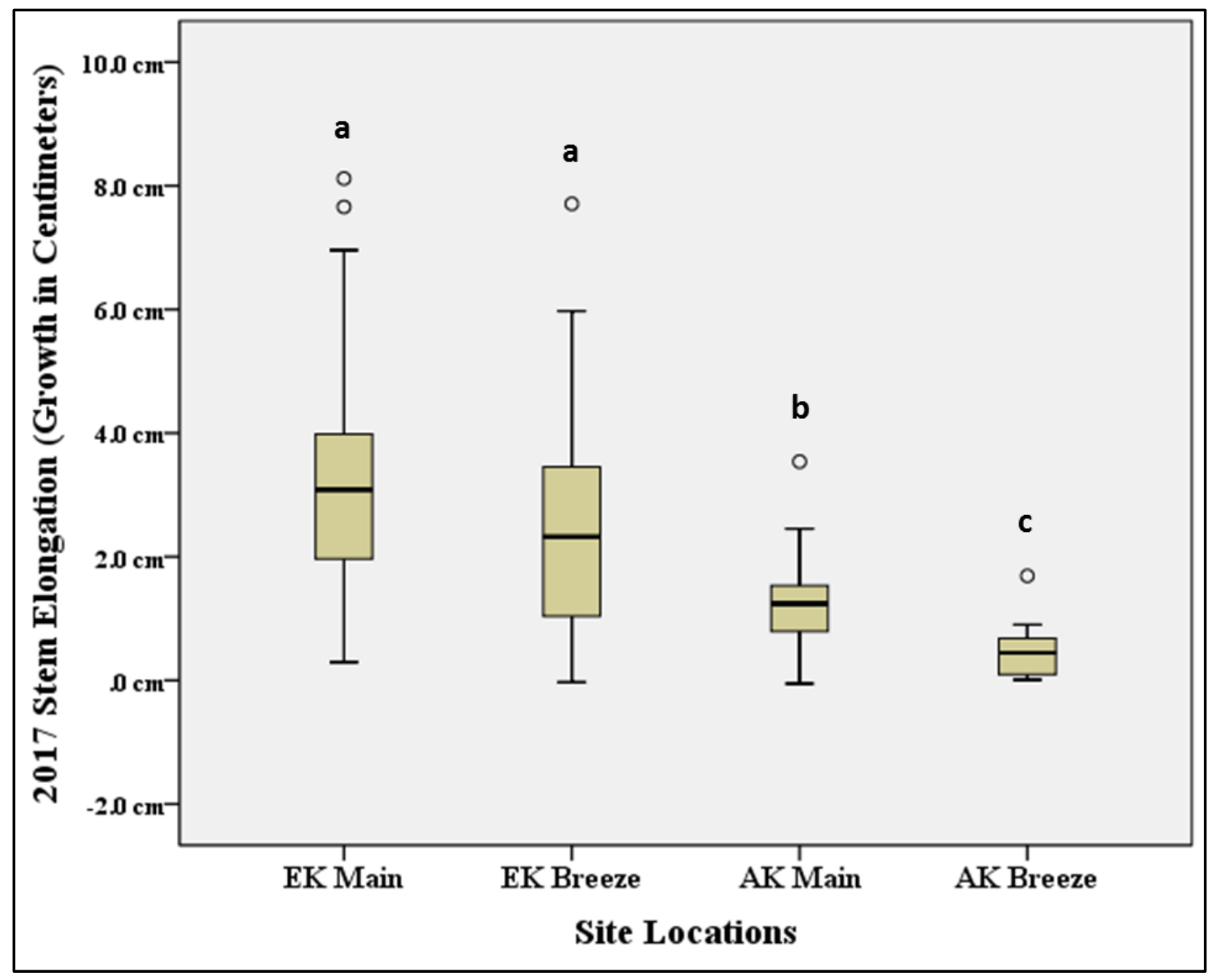


Figure 2.12: Boxplots displaying land elevation $(\mathrm{m})$ at each site. Elevation was recorded for each wild lime planted in the restored and breezeway sites. Different letters above boxplots denote significance. EK Main = Elliott Key Main Restoration Site, EK Breeze = Elliott Key Breezeway Site, AK Main = Adams Key Main Restoration Site, AK Breeze = Adams Key Breezeway Site.

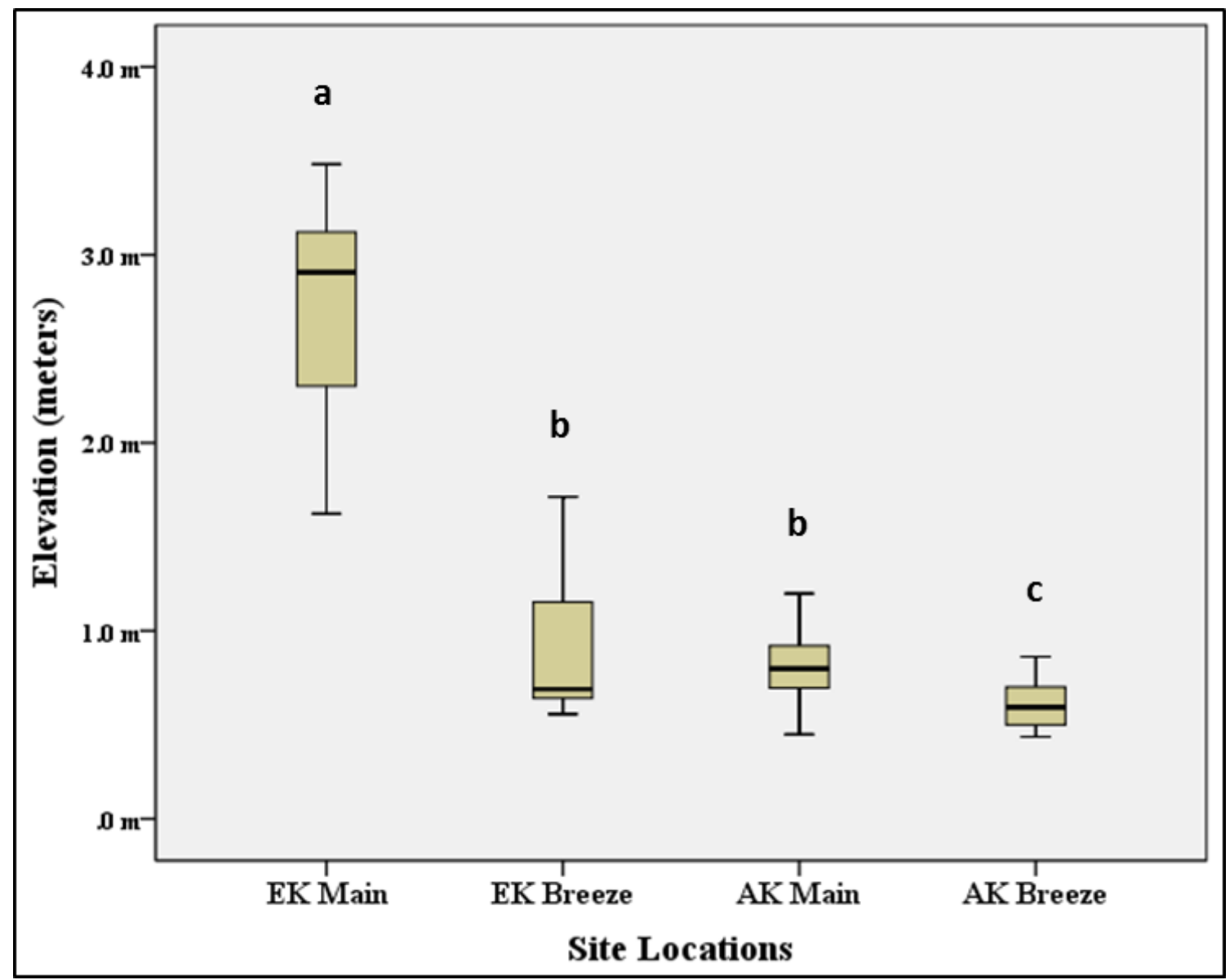


Figure 2.13: Boxplots displaying wild lime stem elongation (growth in $\mathrm{cm}$ ) at each site during 2013. Stem elongation was recorded for each wild lime planted in the restored and breezeway sites. Different letters above boxplots denote significance. Open circles represent suspected outliers; however, extreme outliers were removed. EK Main = Elliott Key Main Restoration Site, EK Breeze = Elliott Key Breezeway Site, AK Main = Adams Key Main Restoration Site, AK Breeze = Adams Key Breezeway Site.

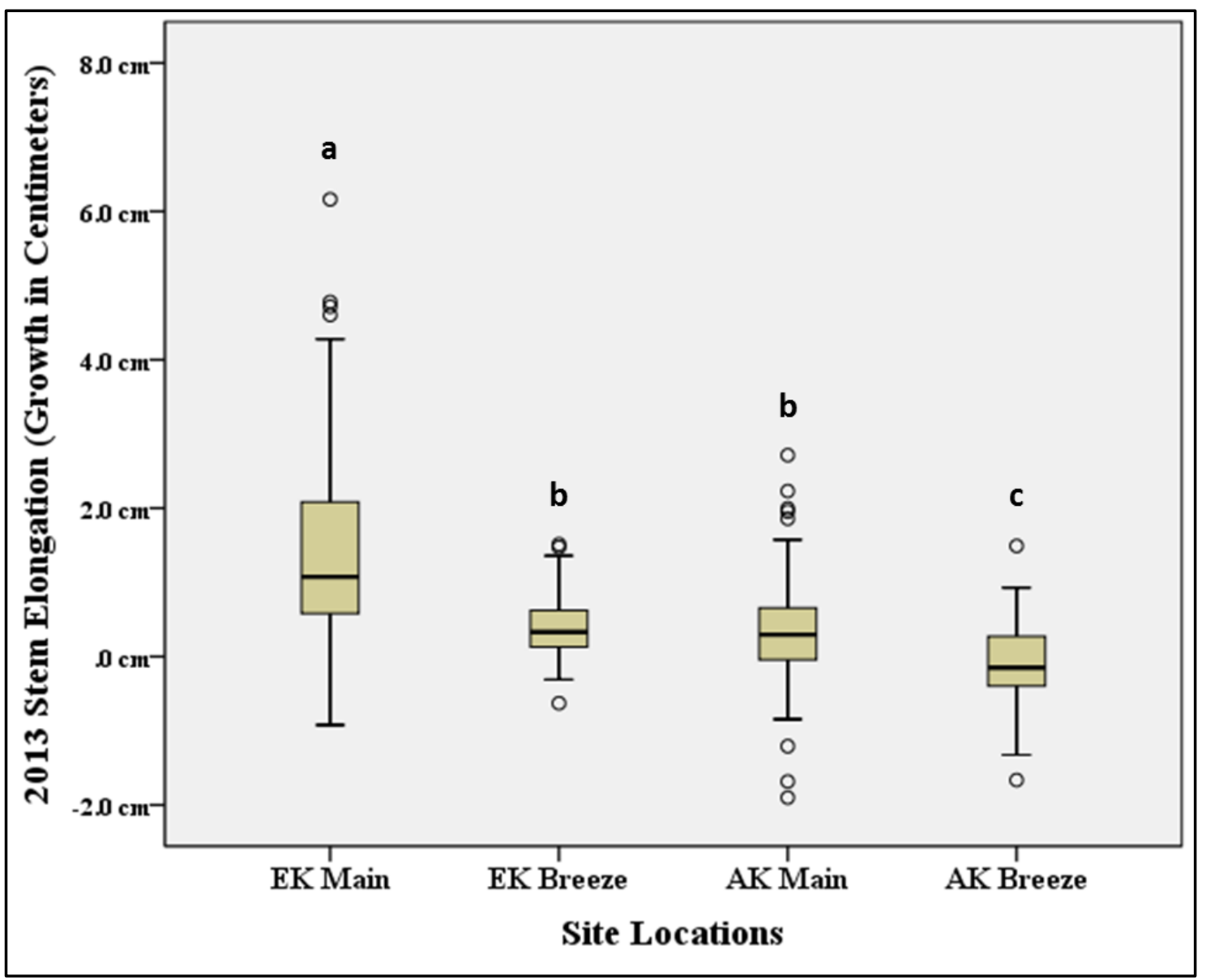


Figure 2.14: Boxplots displaying wild lime stem elongation (growth in $\mathrm{cm}$ ) at each site during 2014. Stem elongation was recorded for each wild lime planted in the restored and breezeway sites. Different letters above boxplots denote significance. Open circles represent suspected outliers; however, extreme outliers were removed. EK Main = Elliott Key Main Restoration Site, EK Breeze = Elliott Key Breezeway Site, AK Main = Adams Key Main Restoration Site, AK Breeze = Adams Key Breezeway Site.

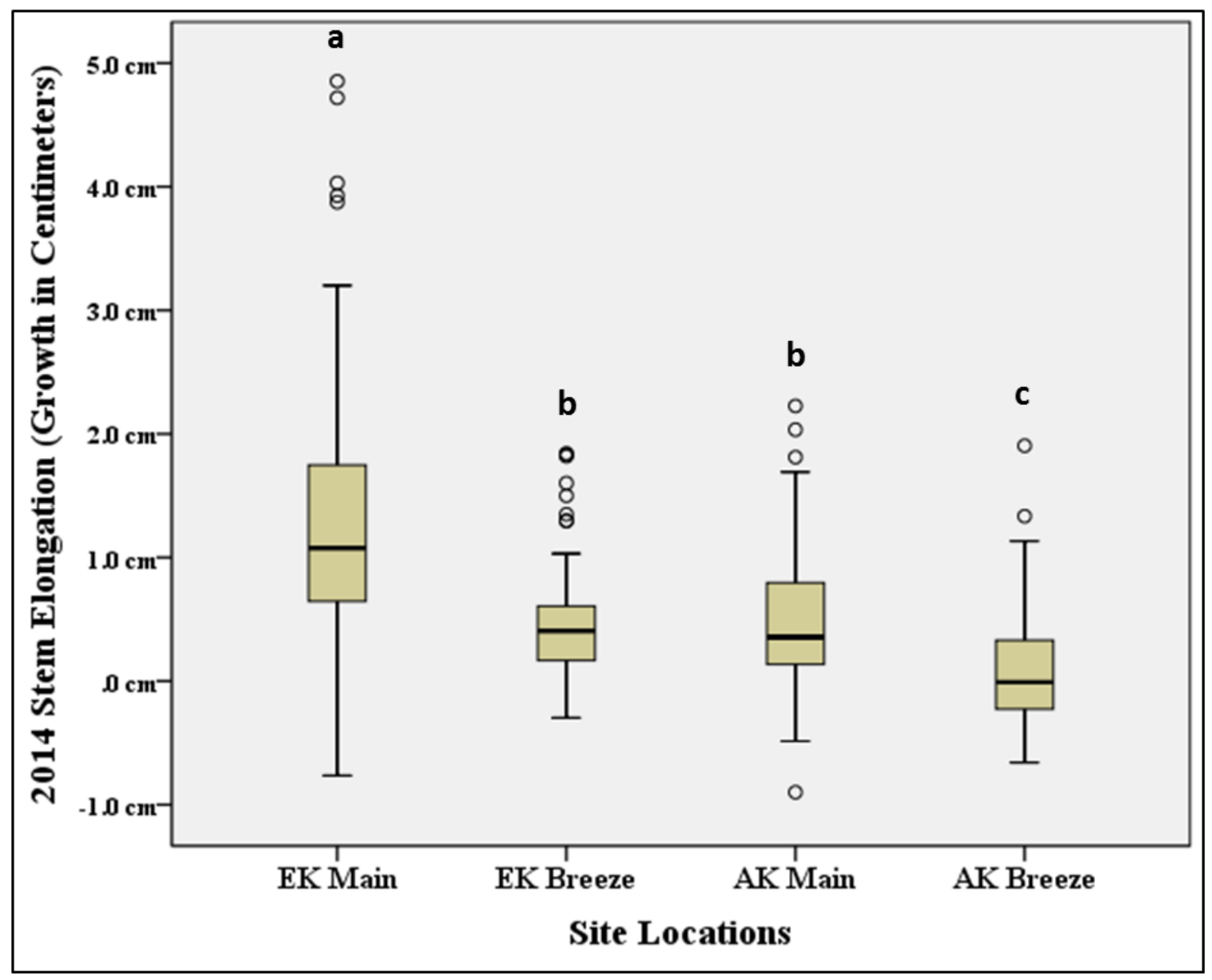


Figure 2.15: The extent of available dry forest habitat at Elliott Key: (1) current sea level, (2) 1 m SLR, and (3) 2 m SLR.

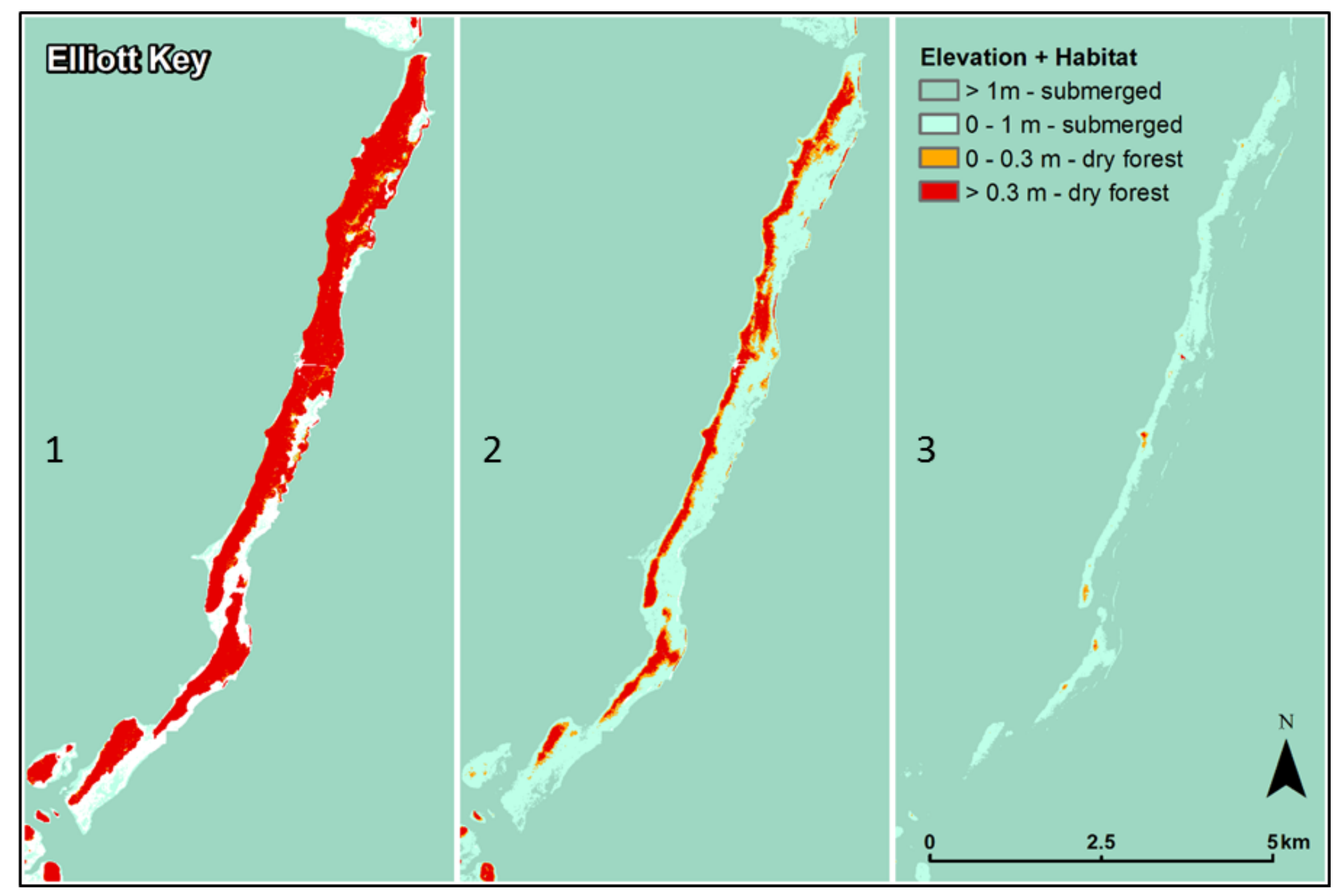


Figure 2.16: The extent of available dry forest habitat at Adams Key: (1) current sea level, (2) $1 \mathrm{~m}$ SLR, and (3) $2 \mathrm{~m}$ SLR.

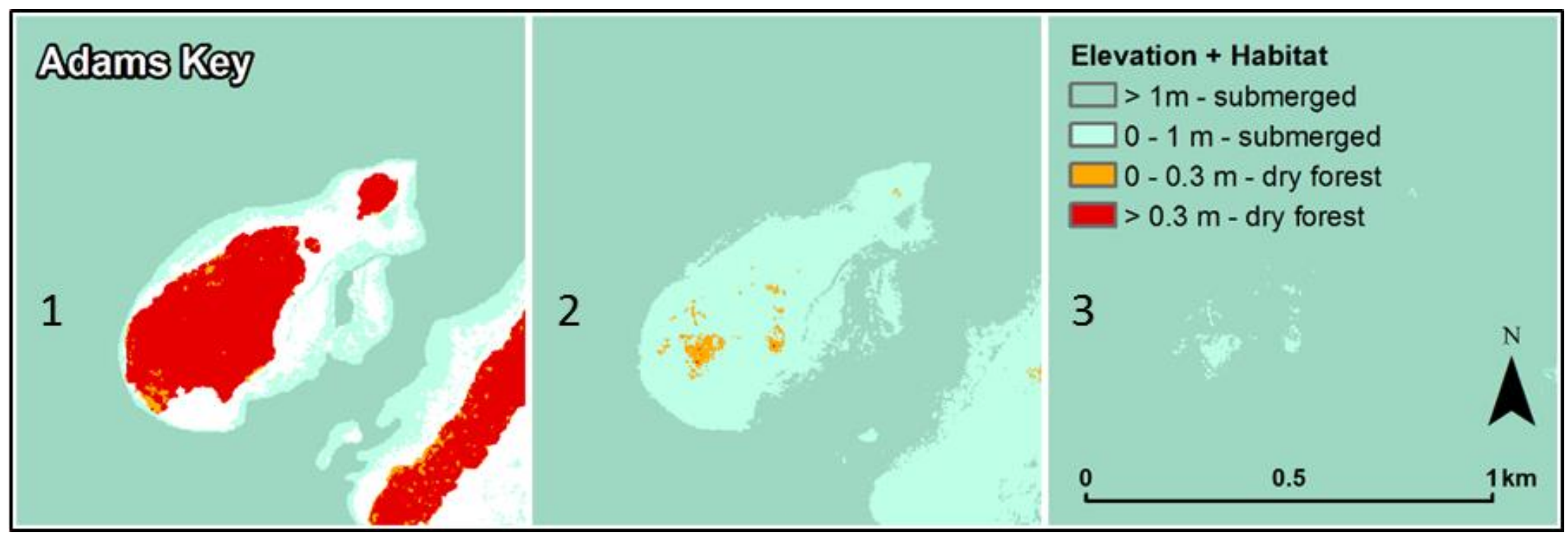


Figure 2.17: The extent of available dry forest habitat at Old Rhodes Key and Totten Key: (1) current sea level, (2) $1 \mathrm{~m}$ SLR, and (3) $2 \mathrm{~m} \mathrm{SLR}$.

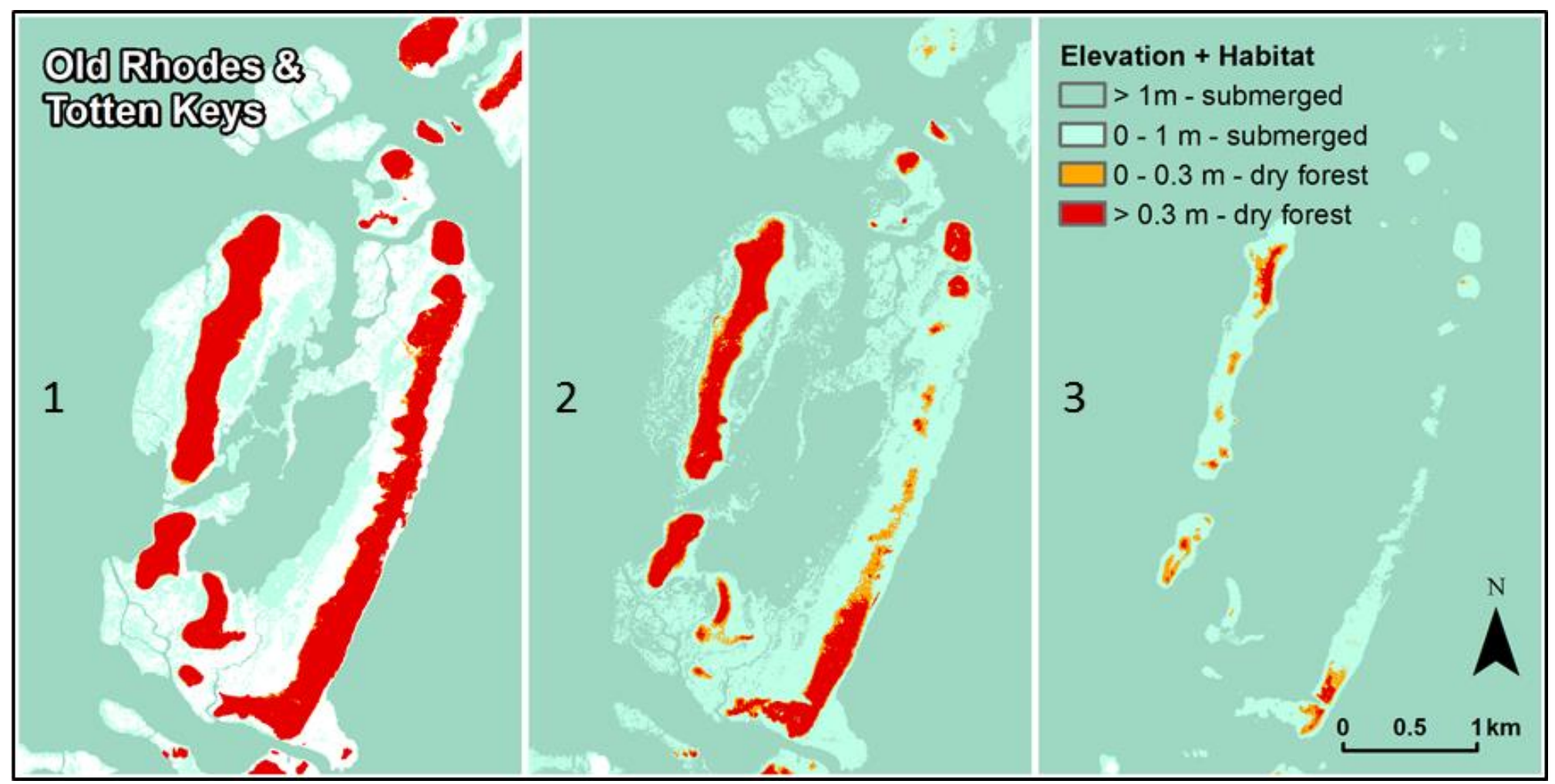


Figure 2.18: The extent of available dry forest habitat in North Key Largo: (1) current sea level, (2) $1 \mathrm{~m}$ SLR, and (3) $2 \mathrm{~m}$ SLR.

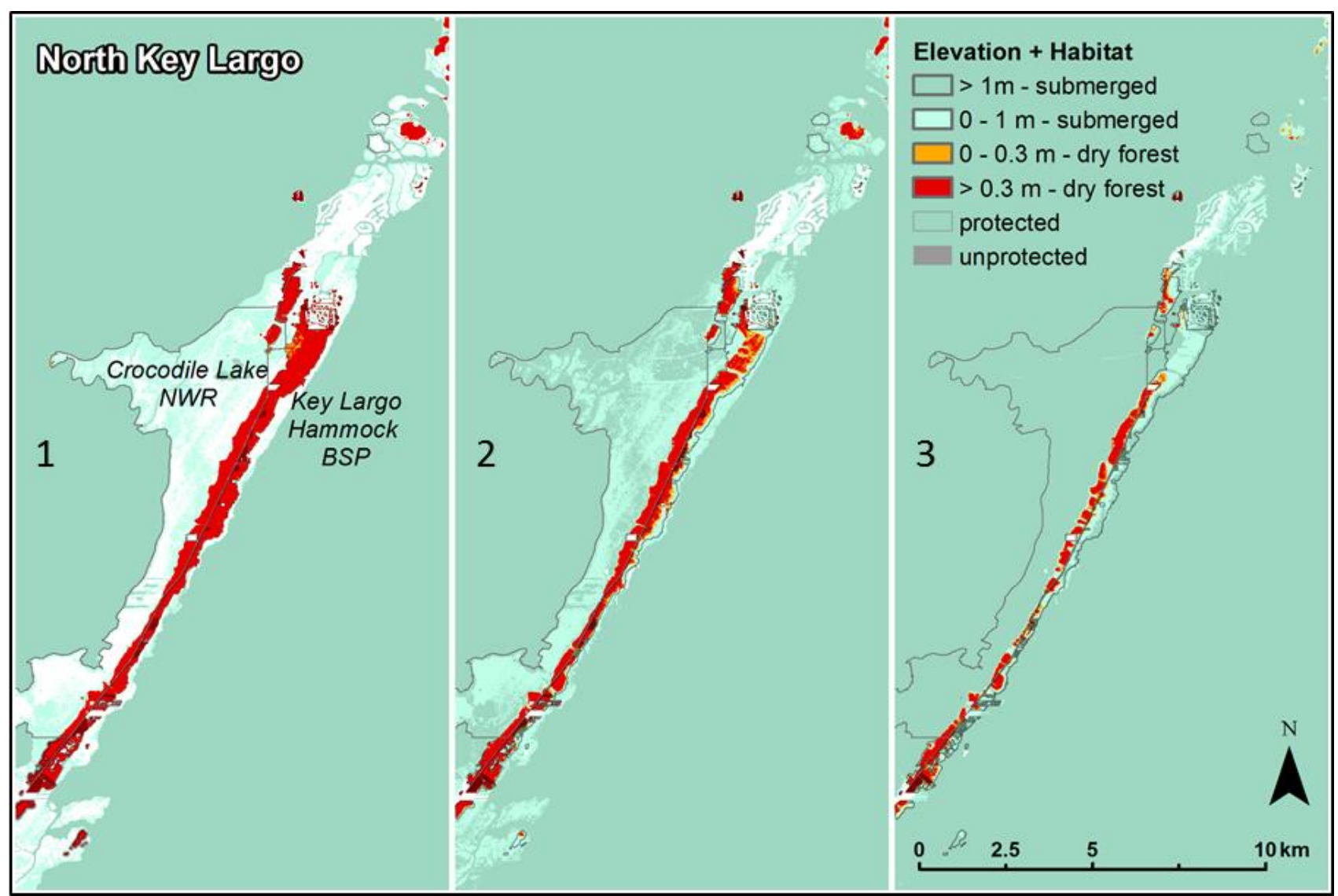


CHAPTER III

MORTAL COMBAT BETWEEN ANTS AND CATERPILLARS: AN OMINOUS

THREAT TO THE ENDANGERED SCHAUS' SWALLOWTAIL BUTTERFLY

(HERACLIDES ARISTODEMUS PONCEANUS) IN THE FLORIDA KEYS, USA

Published in the Journal of Insect Conservation

Clayborn, J., and Koptur, S. 2017. Mortal combat between ants and caterpillars: an ominous threat to the endangered Schaus swallowtail butterfly (Heraclides aristodemus ponceanus) in the Florida Keys, USA. Journal of Insect Conservation, 21(4): 689-702. 


\section{ACKNOWLEDGEMENTS}

We thank Vicente Martinez, Shane Harrington, and Steve Newman for their support and transportation to the islands. The support and cooperation of Biscayne National Park and the National Park Service staff were essential in project development to completion. We greatly appreciate field assistance from many, including Leslie Bayas, Lydia Cuni, Darlene Darrican, Michael Foguer, Tanjim Hossain, Eduardo Ibarra, Craig Perry, Brenda Riveria, Andrea Salas, Adriana Samani, and Yadira Reynaldo. Identification of difficult ant specimens was provided by Mark Deyrup. Paulo Oliveira provided invaluable information and guidance on the ant-caterpillar interaction study. Constructive comments on the manuscript were made by Ian Jones and Scott Zona. Permission to work in Biscayne National Park was granted through the scientific research and collecting permit \# BISC - 2014 - SCI - 0031. This is contribution \#344 to the Tropical Biology Program at Florida International University.

\section{FUNDING}

This study was financially supported through the Zoo Miami Conservation and Research Grant and the Kelly Foundation Botany Scholarship.

\footnotetext{
ABSTRACT

The federally endangered Schaus' swallowtail butterfly (Heraclides aristodemus ponceanus) has reached critically low numbers. Exotic ants are a potential threat to $H$. $a$. ponceanus and other rare butterflies as they can attack immature stages. Ant surveys
} 
conducted in subtropical dry forests in Biscayne National Park documented ant species diversity and relative abundance. A caterpillar predator exclusion experiment using physical barriers in different combinations evaluated caterpillar survivorship of both early and late instar caterpillars exposed to different threats. Ant-caterpillar interactions were also documented by placing caterpillars on plants and observing physical interactions between caterpillars and ants. A total of 1418 ants comprising 25 ant species was captured and identified. In canopies of $H$. a. ponceanus host plants, 243 ants comprising 12 species were found. The four most common ants collected in the host plant canopies were Pseudomyrmex gracilis, Camponotus planatus, Cremastogaster ashmeadi, and Camponotus floridanus. The predator exclusion experiment revealed survivorship was significantly lower for early and late instar caterpillars without any physical barrier, as well as for early instars not protected by a mesh cage. Pseudomyrmex gracilis and $C$. floridanus were more aggressive towards caterpillars in comparison to other ant species; these two species ranked first and second in the "ant danger index" ranking predatory abilities of the four most common ant species. Pseudomyrmex gracilis is a common arboreal exotic ant in Biscayne National Park and presents a major threat to caterpillars during their earliest life stages.

Keywords: Formicidae; Florida Keys; Invasive species; Papilionidae; Predator-prey interactions; Subtropical dry forests 


\section{INTRODUCTION}

Mortality at every life history stage is common for most invertebrates, but for species whose numbers are reduced or reproduction limited to a short time period, heavy predation on immatures can severely threaten their continued existence (Schoener et al. 2001; Beuzelin et al. 2009; Wagner and Van Driesche 2010). The federally endangered Schaus' swallowtail butterfly (Heraclides aristodemus ponceanus) inhabits subtropical dry forest and is known from a limited number of locations. Its occurrence is associated with clumped distributions of torchwood (Amyris elemifera, Rutaceae), the primary larval host plant (Emmel et al. 1988; Jameson 2002; United States Fish and Wildlife Service 2008). Schaus' swallowtail populations in Biscayne National Park (BNP) and North Key Largo (NKL) have declined precipitously over the last 15 years (Salvato 2008; United States Fish and Wildlife Service 2008; Fig. 1). In 2012, two projects were implemented to increase $H$. a. ponceanus population: a captive-breeding program at the University of Florida (Daniels 2014) and the National Park Service (NPS) Schaus' swallowtail habitat enhancement project in BNP. The Schaus' swallowtail habitat enhancement project capitalized upon ongoing projects that required the elimination of invasive plants in particular sections within subtropical dry forests at Elliott and Adams Keys (Whelan 2011; Whelan and Atkinson 2015); over 3000 host plants for the H. a. ponceanus, mostly A. elemifera and Zanthoxylum fagara (Rutaceae), were planted and nurtured in restoration sites at Elliott and Adams Keys. Despite a bottom-up approach (increased food source) to bolster H. a. ponceanus populations (Jameson 2002; Dennis et al. 2004; Mathew and Anto 2007), investigations of top-down effects on a rare butterfly concentrated in BNP and NKL are nonexistent (Didham et al. 2007; Salvato 2008; United 
States Fish and Wildlife Service 2008). Predation by invertebrates, such as ants, can thwart the recovery process (Schoener et al. 2001; Wagner and Van Driesche 2010; Lach et al. 2016). Specifically, most ants are generalist and opportunistic foragers, numerically dominant in subtropical and tropical ecosystems, and active day and night foragers (Carroll and Janzen 1973; Jeanne 1979; Brown 2000; Floren et al. 2002). The ecological impacts of exotic ants inhabiting the islands are unknown, despite their ubiquitous presence in the forests (United States Fish and Wildlife Service 2008; Moreau et al. 2014).

Exotic ants can negatively affect other organisms through predation, competition, and alteration of local habitats (Williams 1994; Vitousek et al. 1996; Mack and D’Antonio 1998; Holway et al. 2002; Lach and Hooper-Bùi 2010; Lach et al. 2016). Prolific exotic ants can become invasive in new territories with novel food resources, reduced interspecific and intraspecific competition, release from natural enemies, and enhanced physiologic abilities to thrive in disturbed environments (Holway et al. 2002; Krushelnycky et al. 2010; Lach et al. 2016; Calcaterra et al. 2016). Approximately 37 exotic ant species have been identified in the Florida Keys, including three species on the top 100 world's worst invasive alien species list: the big-headed ant (Pheidole megacephala), the little fire ant (Wasmannia auropunctata), and the red imported fire ant (Solenopsis invicta) (Lowe et al. 2000; Moreau et al. 2014). Few studies have investigated direct and indirect impacts of exotic ants on native flora and fauna in the Florida Keys, with the exception of red imported fire ants; a laboratory study confirmed that red imported fire ants are highly efficient predators of all immature life stages of the 
giant swallowtail butterfly (Heraclides cresphontes), which were used as a surrogate species for H. a. ponceanus (Forys et al. 2001).

Solenopsis invicta has been considered a potential threat to $H$. a. ponceanus because of its aggressive nature (Forys et al. 2001). Solenopsis invicta displace native species in disturbed environments; they are efficient predators and scavengers, consuming vulnerable organisms including ticks, caterpillars, beetle grubs, and even mosquito larvae in moist substrate (Porter and Savignano 1990; Forys et al. 2002; Zettler et al. 2004; Allen et al. 2004; Beuzelin et al. 2009). Solenopsis invicta form large colonies that can monopolize disturbed sites adjacent to subtropical dry forests (Forys et al. 2001, 2002; Tschinkel 2006). In south Florida, they are unlikely to colonize interior parts of subtropical dry forests because they are mainly habitat-restricted to human-modified, disturbed sites (Tschinkel 1988, 2006; King and Tschinkel 2006).

The exotic graceful twig ant (Pseudomyrmex gracilis), is arboreal and also poses potential risk to rare butterflies as the ants search for prey on shrubs and trees (Saarinen and Daniels 2006; Wetterer 2010). An opportunistic, solitary forager, P. gracilis mainly scavenges for food in its natural range (Mexico, Central, and South America; Whitcomb et al. 1972). In south Florida, its predatory behavior is deleterious to rare and vulnerable invertebrate species because these ants are physically larger than the other native Pseudomyrmex ant species in south Florida (Wetterer 2010; Deyrup 2016); years earlier, Deyrup et al. (2000) warned that the graceful twig was a "possible ecological villain" in Florida. Pseudomyrmex gracilis is a common pest on shrubs and trees in both urban and natural environments (MacGown and Hill 2010). 
The exotic little fire ant (Wasmannia auropunctata) is a small stinging ant that can overwhelm invertebrate prey through aggressive recruitment (Wetterer and Porter 2003). In BNP, W. auropunctata has been collected on both trees and ground. The little fire ant inhabits both disturbed and intact forests in BNP. Wasmannia auropunctata and $P$. gracilis are native to Mexico and Central America (Wetterer and Porter 2003; Wetterer 2010). All three exotic ant species are native to South America (Buren et al. 1974; McGlynn 1999).

The limited range of Heraclides aristodemus ponceanus, reduced habitat, and exposure to natural extreme weather events may not allow its immature stages to withstand exotic and native ant predation over time (Schoener et al. 2001; United States Fish and Wildlife Service 2008), limiting the number of adult butterflies of this endangered species. The current government management protocol for $H$. a. ponceanus reported exotic ants as major threats to the eggs and larvae (United States Fish and Wildlife Service 2008). Here we address potential impacts ants may have on caterpillars that use A. elemifera and Z. fagara as larval host plants. We want to know: (1) What ant species forage in the canopies of A. elemifera and Z. fagara in BNP?; (2) Are the most common ant species native or exotic?; (3) Are crawling or non-crawling predators greater threats to Heraclides caterpillar species?; and (4) How do interactions between the most common ant species and Heraclides caterpillar species compare? 


\section{METHODS}

\section{Site description}

Biscayne National Park (BNP) became a national park in 1980, with most of its area (95\%) comprised of marine environments. The terrestrial area consists of a narrow strip of land on the mainland and 42 islands, most of which is dominated by mangrove forest (2400 ha). Coastal subtropical dry forests, critical habitat for H. a. ponceanus, make up only 723 ha (23\%) of the terrestrial lands (Whelan et al. 2013). Elliott Key $\left(25^{\circ} 27^{\prime} 12.39^{\prime \prime} \mathrm{N}, 80^{\circ} 11^{\prime} 39.05^{\prime \prime} \mathrm{W}\right)$, the largest island, is roughly 7 miles long, and nearly a mile wide, with subtropical dry forests covering 68 percent of the area (Whelan et al. 2013). On Adams Key $\left(25^{\circ} 23^{\prime} 52.34^{\prime \prime} \mathrm{N}, 80^{\circ} 14^{\prime} 05.67^{\prime \prime} \mathrm{W}\right)$, subtropical dry forests account for $43 \%$ of the area.

South Florida has a distinct wet-dry season: the rainy season typically starts early June and continues through October; however, dry spells can occur in July and part of August (Snyder et al. 1990). The univoltine H. a. ponceanus emerges from its chrysalis typically in May with a flight season lasting until mid-June; occasionally, a second emergence happens mid-August and September depending on the amount of rain (Loftus and Kushlan 1984; Emmel et al. 1988; Minno and Emmel 1993). Heraclides aristodemus ponceanus have been reported flying as early as April. Currently, the largest population of H. a. ponceanus inhabits BNP, particularly Elliott and Adams Keys (Minno 2015).

\section{Ant survey}

Ant surveys were conducted for 1 year (December 2013-November 2014) in the subtropical dry forest at Elliott and Adams Keys. Ants were collected using pitfall traps 
to characterize relative species abundance and diversity (Wang et al. 2001; Andersen et al. 2002); each trap was a $50 \mathrm{~mL}$ plastic test tube filled with $20 \mathrm{~mL}$ of lemon-scented, soapy water solution, with one hole $(1.5 \mathrm{~cm}$ diameter) drilled into each screw-on cap to protect against adverse weather conditions (Wang et al. 2001; Ribas et al. 2003; Lubertazzi and Tschinkel 2003). Each sampled area was selected based on the presence of A. elemifera and Z. fagara, along a North-South gradient at Elliott Key, and an EastWest gradient at Adams Key, differing because of the islands' overall shapes. Non-host trees in the forest, 10-15 $\mathrm{m}$ away from each A. elemifera and Z. fagara sampled, were also sampled. Four pitfall traps were placed on or near each tree: one at the base, one on the trunk ( $2 \mathrm{~m}$ above the ground), and two in the canopy. Pitfall traps were dispersed throughout the sampled areas every month, collecting crawling insects for 5 days. Afterwards, collected ants were preserved for subsequent determination. A total of 296

pitfall traps was deployed on 74 trees at Elliott and Adams Keys. One hundred forty-eight traps were placed in tree canopies, 74 were placed on the trunk, and 74 were partially buried in the ground adjacent to the base of each tree. Thirty A. elemifera, 14 Z. fagara, and 30 non-host trees were sampled during the study.

\section{Caterpillar predator exclusion experiment}

A $2 \times 6$ factorial design with six treatments was employed to compare the effects of crawling and non-crawling predators against caterpillars of two different sizes (modified from Koptur et al. 1998; Cuautle and Rico-Gray 2003; Mestre et al. 2016). This experiment was conducted from March 2014 to November 2014. Using soft forceps, early $(<1.5 \mathrm{~cm})$ and late $(>3 \mathrm{~cm})$ instar H. cresphontes caterpillars (used as a surrogate 
for H. a. ponceanus caterpillar) were gently placed on Z. fagara in the forest at Elliott Key only; logistical constraints did not allow this experiment to be adequately conducted at Adams Key. Despite H. a. ponceanus preference for A. elemifera, they also utilize $Z$. fagara as a secondary host plant (Jameson 2002; United States Fish and Wildlife Service 2008). The majority of $H$. cresphontes caterpillars, raised in an outdoor nursery at Florida International University for both the caterpillar predator exclusion experiment and ant caterpillar interaction study, were fed Z. fagara leaves, due to both logistical and financial constraints of maintaining an adequate supply of A. elemifera.

Treatments applied to caterpillars were: (1) no barriers (no tanglefoot/no cage), (2) tanglefoot present/no cage, (3) no tanglefoot/cage with holes present, (4) tanglefoot present/cage with holes present, (5) no tanglefoot/cage present, and (6) tanglefoot present/cage present. Tanglefoot ${ }^{\circledR}$ is a non-toxic, non-drying, sticky resin that creates a barrier and deters crawling insects from crossing it. Cages were made from recycled 32 oz sports drink bottles that were cut open, shaped into a frame, and placed over a wild lime branch. Ultra-fine cotton cheesecloth was placed over the sports drink bottle frame and sealed with staples and white zip ties. One caterpillar was placed on a branch inside the cage or without a cage; for early instar caterpillars, each treatment had 16 replicates; for late instar caterpillars, each treatment had 15 replicates. Some treatments required holes in the mesh cage; therefore, six holes $(\mathrm{r}=1.5 \mathrm{~cm})$ were cut into the mesh. Each individual Z. fagara tree received a randomly assigned assortment of 6-8 treatments spread across different branches. Each Z. fagara tree was thoroughly scrutinized for caterpillar presence or absence after 5 days and recorded to determine survivorship for each treatment. No Z. fagara tree was used more than once during the experiment. 


\section{Ant-caterpillar interaction study}

Ant interactions with both early $(<1.5 \mathrm{~cm}$ in length) and late $(>3 \mathrm{~cm})$ instar caterpillars were conducted in the forests at Elliott and Adams Keys from April 2014 to October 2014. Heraclides cresphontes caterpillars were again used as surrogate species, as they also use A. elemifera and Z. fagara as host plants in BNP, and interact with the same ant species. Foraging ant locations were selected based on relative abundance (determined from previous ant surveys) in the canopy of A. elemifera and Z. fagara. An individual caterpillar was placed on a leaf (A. elemifera and Z. fagara) and allowed to settle before interacting with ants. Observations ended after 10 min upon first contact, unless the caterpillar was removed before that time (Oliveira et al. 1987; Bächtold et al. 2012; Sendoya and Oliveira 2015). Observers timed each interaction trial from the first contact between ant and caterpillar. The caterpillar's fate was reported after each trial. Caterpillar mortality was defined as removal by ant species or caterpillar leaf abandonment (dropping from the leaf to avoid ant harassment). No caterpillars or ants were used more than once during this study; for early instar caterpillars, 16 trials each were conducted with Camponotus floridanus and Camponotus planatus, 15 trials with $P$. gracilis, and 10 trials with Cremastogaster ashmeadi; for late instar caterpillars, 15 trials each were conducted with $C$. floridanus, $C$. planatus, and $P$. gracilis, and 11 trials with C. ashmeadi.

\section{DATA ANALYSIS}

A generalized linear model (negative binomial with log link to account for overdispersion) was used to model the frequency of $P$. gracilis on trees. The negative 
binomial model was selected as the best-fit model to account for overdispersion and true zeros. Model comparisons (poisson and negative binomial) were analyzed using the program IBM® SPSS $®$ Statistics 22 (2013).

A contingency table analysis was performed on data collected from the caterpillar predator exclusion experiment. Adjusted standardized residuals (Z-scores) calculated from the contingency table were transformed by squaring them to obtain Chi square values. Afterwards, the Chi square values were used to calculate exact $\mathrm{p}$ values with the significance function group in SPSS. Exact $\mathrm{p}$ values were compared to the adjusted $\alpha$ value to determine significance. Adjusted $\alpha$-values were calculated by dividing the accepted $\alpha$-value of 0.05 by the total number of factors (12) which equaled 0.004167 . Exact $\mathrm{p}$ values equal to or less than the adjusted $\alpha$-value were considered significant.

A Shapiro-Wilk test for normality was performed on the discovery time for the antcaterpillar interaction study. A one-way ANOVA was performed followed by the Fisher's Least Significant Difference post-hoc test. All data analyses reported above were conducted using IBM® SPSS ${ }^{\circledR}$ Statistics 22 (2013).

An Ant Danger Index (ADI) was generated to rank the predatory abilities of ants against caterpillars. Each ant species was given an ADI score based on relative abundance in tree canopies (A. elemifera and Z. fagara), average time to first interaction with caterpillar, rate of ant recruitment, and ant-caterpillar interactions leading to caterpillar mortality. The total score for each ant species was divided by 25 (the maximum score an ant species could receive) and then multiplied by 100 (percentage of total possible score) for their final ADI score, which ranged from 0 to 100 , allowing the species to be ranked (Possley et al. 2016). 


\section{RESULTS}

\section{Ants on and around plants}

Twenty-five ant species were collected in pitfall traps on trees at Elliott and Adams Keys in BNP (Table 1; Fig. 2), a total of 1418 individual ants. Taxa were determined with help from Mark Deyrup (Archbold Biological Station, Venus, Florida). Twelve ant species (243 total ants) were collected in canopies of torchwood and wild lime (Table 2;

Fig. 3); the four most common of these were P. gracilis, C. planatus, C. ashmeadi, and $C$. floridanus. The most abundant ant collected in canopies of A. elemifera and Z. fagara was $P$. gracilis, representing $35 \%$ of the total ants collected; $C$. planatus was second at $21 \%$; C. ashmeadi was third at 18\%; and C. floridanus was fourth at $13 \%$ (Fig. 3). These four ant species represented $87 \%$ of collected ants in canopies of A. elemifera and $Z$. fagara. Both $P$. gracilis and $C$. planatus are exotic ant species in south Florida (Moreau et al. 2014; Deyrup 2016).

The frequency of $P$. gracilis individuals collected on three different groups of trees was compared using a generalized linear model (negative binomial with log link). This ant species was most frequent on A. elemifera $($ mean $=1.590, \mathrm{SD}=2.080)$, followed by Z. fagara $($ mean $=1.450, \mathrm{SD}=1.927)$, and then non-host trees $($ mean $=0.990, \mathrm{SD}=$ 1.421). The omnibus test (compares the fitted model against the intercept only model) for the negative binomial model was not significant $(\chi 2=4.291, \mathrm{df}=2$, $\mathrm{p}$ value $=0.117$; Table 3). However, individual parameters (tree groups) were investigated to determine significance using non-host trees as the baseline group to compare the frequency of $P$. gracilis collected for each tree group. Only P. gracilis collected on A. elemifera (the 
primary host tree for $H$. a. ponceanus) was significantly higher than non-host trees $(\chi 2=$ 4.119, $\mathrm{df}=1, \mathrm{p}$ value $=0.042 ;$ Table 3; Fig. 4).

\section{Caterpillar predator exclusion experiment}

Exclusion experiments revealed that early instar caterpillars were vulnerable to both crawling and non-crawling predators. Contingency table analysis revealed that survivorship, defined as caterpillar present on leaf, was significantly lower for early instar caterpillars in treatments with no barriers $(18.8 \%$; exact $\mathrm{p}$ value $=0.000003<$ adjusted $\alpha$ value $=0.004160)$, with tanglefoot only $(31.3 \%$; exact $p$ value $=0.000465<$ adjusted $\alpha$ value $=0.004160)$, and for cages with holes and tanglefoot absent $(37.5 \%$; exact $\mathrm{p}$ value $=0.002700<$ adjusted $\alpha$-value $=0.004160$ ), compared to the other barrier treatments (Table 4; Fig. 5). For the larger caterpillars, survivorship was only significantly lower when there were no barriers at all $(33.3 \%$; exact $\mathrm{p}$ value $=0.001374<$ adjusted $\alpha$-value $=$ 0.004160; Table 4; Fig. 5).

\section{Ant-caterpillar interactions}

Pseudomyrmex gracilis $(\mathrm{n}=30$ trials; mean $=56.533, \mathrm{SD}=32.324, \mathrm{p}<0.001)$

discovered caterpillars significantly faster than did C. planatus $(\mathrm{n}=31$ trials;

mean=115.806, $\mathrm{SD}=58.579), C$. ashmeadi $(\mathrm{n}=21$ trials; mean= $123.524, \mathrm{SD}=64.697)$, and $C$. floridanus $(\mathrm{n}=29$ trials; mean $=122.690, \mathrm{SD}=87.015$; Fig. 6). Crematogaster ashmeadi $(\mathrm{n}=21$ trials; mean $=0.429, \mathrm{SD}=0.148)$ and $C$. floridanus $(\mathrm{n}=29$ trials; mean= $0.345, \mathrm{SD}=0.614)$ occasionally recruited workers to combat caterpillars; $P$. gracilis and C. planatus did not recruit workers when interacting with caterpillars (Fig. 7). Overall, 
early instar caterpillars had higher rates of mortality compared to late instar caterpillars. Early instar caterpillars suffered the most damage when interacting with $P$. gracilis $(\mathrm{n}=15$ trials, $86.7 \%$ mortality); late instar caterpillars successfully foiled $P$. gracilis advances (n $=15$ trials, $0 \%$ mortality). Early instar caterpillar mortality was moderately high with $C$. floridanus ( $\mathrm{n}=16$ trials, $56.3 \%$ mortality); these were the only ant species tough enough to remove or force some late instar caterpillars to completely abandon the leaf $(n=15$ trials, $40 \%$ mortality). Crematogaster ashmeadi attacked only a few early instar caterpillars during the trials ( $\mathrm{n}=10$ trials, $20 \%$ mortality) and were ineffective in harming late instar caterpillars ( $\mathrm{n}=11$ trials, $0 \%$ mortality). Finally, $C$. planatus demonstrated no aggressive encounters with either early ( $\mathrm{n}=16$ trials, $0 \%$ mortality) or late instar ( $\mathrm{n}=15$ trials, $0 \%$ mortality) caterpillars (Table 5).

The Ant Danger Index ranked the predatory abilities of $C$. ashmeadi, $C$. planatus, $C$. floridanus, and $P$. gracilis against caterpillars that utilized A. elemifera and Z. fagara as host plants; since all four ant species represented $87 \%$ of the total sampled ants collected in canopies of A. elemifera and Z. fagara, only these ants were compared. Pseudomyrmex gracilis received the highest final score at 52, and C. floridanus had the next highest score at 40; both C. ashmeadi and C. planatus received lower scores of 24 (Table 6).

\section{DISCUSSION}

Ants are important predators in tropical forest ecosystems (Jeanne 1979; Smiley 1985; Tobin 1995; Floren et al. 2002). Their abundance and active foraging day and night presents chronic passive and aggressive encounters with caterpillars (Lopez and Potter 2000; Seifert et al. 2016). Many factors, however, determine whether ant species will 
have beneficial, neutral, or negative effects toward caterpillars (Buckley 1987; Kaminski et al. 2010; Mestre et al. 2016). With the exception of those Lepidoptera whose caterpillars have mutualistic relationships with ants, early instar and small caterpillars are negatively associated with invertebrate predators, especially in subtropical and tropical ecosystems (Jeanne 1979; Montllor and Bernays 1993; Reavey 1993; Floren et al. 2002; Sam et al. 2015). Late instar and large caterpillars are negatively associated with birds especially in temperate ecosystems (Heinrich 1993; Reavey 1993; Remmel et al. 2009). Generalist and specialist parasitoids typically attack eggs, caterpillars and even the pupal stage (Weseloh 1993; Gentry and Dyer 2002; Stireman et al. 2009). We did not investigate either bird or parasitoid impacts during this study, which are other areas of research interest for future studies (Gentry and Dyer 2002; Timms et al. 2016).

Results from the predator exclusion experiment demonstrated that early instar caterpillars were less likely to survive when exposed to crawling and non-crawling predators (Fig. 5), but this conclusion was based on several assumptions. For example, caterpillar absence assumed caterpillar mortality, though caterpillars can drop off the leaf or migrate to another area if conditions are less than desirable (Castellanos et al. 2015). We accounted for this by scanning the entire tree after each trial to see if the caterpillar relocated to a different section on the tree. Early instar caterpillars experienced reduced survivorship in treatments when crawling insects gained access to them (no tanglefoot and holes in the cage), with the exception of tanglefoot present and no cage to protect the caterpillar. This outcome suggests that crawling predators such as ants, beetles, true bugs, spiders, and even lizards play a significant predator role. Late instar caterpillars had high survivorship for all treatments, except the treatment without barriers. As mentioned 
above, late instar caterpillar absence assumed caterpillar mortality, despite caveats including migration to pupate, foraging for more food resources, or leaf abandonment. Overall, results were consistent with past studies that concluded early instar (small) caterpillars suffered higher mortality than late instar (bigger) caterpillars (Tilman 1978; Watanabe 1981; Sam et al. 2015). The predator exclusion experiment elucidated coarse predator impacts (caterpillars present or absent) without defining specific causes (what agents were the cause of caterpillar mortality or disappearance). Since ants are ubiquitous in the forest in BNP, we assumed they would interact with caterpillars more frequently than other predators, though certainly other invertebrates (wasps, parasitoids, spiders), birds, and other vertebrates (lizards, treefrogs) may also play a role (Jeanne 1979; Sam et al. 2015; Seifert et al. 2016).

Ants, particularly $P$. gracilis, may pose a significant threat to butterfly eggs and larvae, but butterflies have developed ways to cope with such predators (Morais et al. 1999). Heraclides aristodemus ponceanus and other closely related swallowtails physically resemble lizard and bird droppings, an adaptation that may thwart vertebrate predation for predators that rely upon visual detection (Minno and Emmel 1992). Accordingly, invertebrate predators are more likely deterred by defensive tactics such as offensive chemicals stored in the caterpillars' osmeteria (defensive organs that discharge a noxious odor), as well as physical head butts by the caterpillar to its attacker(s) (Fig. 8), and the most extreme response, leaf abandonment, where some caterpillars silk off (with a strand of silk that enables them to climb back up once the threat is gone) or directly drop off the leaf to escape predators (Honda 1983; Nafus 1993; Salazar and Whitman 2001; Gentry and Dyer 2002; Frankfater et al. 2009). All these defensive strategies may 
be less effective against aggressive, exotic ants (Forys et al. 2001; Lach et al. 2016). Introduced predators can overwhelm and eat their prey to extinction when the prey population reaches dangerously low numbers; predation effects are exacerbated when prey populations are constrained by other environmental factors such as habitat loss, inclement weather, climate change, and local anthropogenic impacts (Schoener et al. 2001; Lach et al. 2016). The most common ant on A. elemifera and Z. fagara in BNP was P. gracilis, which aggressively attacked and removed early instar caterpillars (86.7\%; Table 5). Field observations revealed that sheer caterpillar size was a fair defense against these ants; in fact, head butting and everting osmeteria were also effective against more aggressive individual ants. As these experiments employed a surrogate species of caterpillars, $H$. cresphontes, we do not know how often ants of this species encounter $H$. a. ponceanus caterpillars in the field. Continued research should take steps to quantify those encounters.

Camponotus floridanus ants did attack some late instar caterpillars (40\%), but the attacks did not result in direct mortality. Harassed late instar caterpillars initially defended themselves, until more ants were recruited to attack. Eventually, the late instar caterpillar abandoned its leaf, falling to lower leaves or to the ground. Conversely, $C$. floridanus ants attacked and killed early instar caterpillars by simply carrying them away. Overall, late instar caterpillars are more equipped to defend themselves and avoid direct predation from these ants, the most common native ant species foraging on A. elemifera and Z. fagara.

Amyris elemifera should be inspected for the presence of extrafloral nectaries (EFNs) since $P$. gracilis was significantly more frequent on them compared to Z. fagara and non- 
host trees (Fig. 4). In the same plant family, Z. fagara does have minute EFNs (Koptur 1992), which might explain the higher frequency of $P$. gracilis on both A. elemifera and Z. fagara. Many non-host trees sampled at Elliott and Adams Keys also have EFNs, including blackbead (Pithecellobium keyense), catclaw blackbead (P. unguis-cati), and soldierwood (Colubrina elliptica), but presence of EFNs on trees does not solely determine whether ants will actively forage on them. The presence of exudate-producing insects can also encourage ants to occupy and forage on plants (Sendoya et al. 2016). This project did not record and quantify the presence and abundance of exudate producing insects, but future investigations should incorporate plant attractiveness to ants and other invertebrate predators due to intrinsic and extrinsic plant characteristics. Numerous studies conducted on ant attracting plants have demonstrated some caterpillar species are well-equipped to thwart ant attacks using behavioral, chemical, and physical means (Oliveira and Freitas 2004; Bächtold et al. 2012; Sendoya and Oliveira 2015).

Biscayne National Park and North Key Largo are the last remaining sanctuaries for the federally endangered H. a. ponceanus (Daniels 2014; Minno 2015); Biscayne National Park supports the largest population of H. a. ponceanus (Minno 2015). The Schaus' swallowtail habitat enhancement project was initiated to bolster H. a. ponceanus populations on Elliott and Adams Key through planting thousands of native trees interspersed with nectar plants in the restored sites. Additional host plants, supplemented with captive-bred $H$. a. ponceanus reintroductions being conducted by scientists and staff at the University of Florida, present options for a better future for H. a. ponceanus in BNP (Daniels 2014). 
Resource managers and scientists should continue to monitor H. a. ponceanus populations over time (Minno 2015). Long-term quantitative research (including video surveillance) may be necessary to assess potential risks imposed by exotic ants. High definition video surveillance cameras can capture predation events in natural settings day and night over an extended period of time to more accurately assess the effects of predator (particularly ants) interactions with all immature stages of $H$. a. ponceanus (Grieshop et al. 2012). In BNP, P. gracilis is the most potentially destructive force against Heraclides species on A. elemifera and Z. fagara. These negative impacts probably extend to other caterpillar species, since this exotic ant is omnipresent and an opportunistic forager (Wetterer 2010).

Different predators may be more or less deleterious at different life-history stages of butterflies (Nafus 1993; Seifert et al. 2015; Sam et al. 2015). Results obtained from these studies can guide future efforts to re-establish rare butterflies in areas they were once known to exist, by utilizing different techniques for protecting eggs and larvae of endangered butterflies at different life stages in reintroduction programs. A better understanding of which predators interact with butterfly eggs and larvae in natural habitats can help scientists determine suitable habitats for captive-reared butterflies before release. If exotic ants are found to be significantly deleterious to butterflies (Lach et al. 2016), then potential sites should be surveyed for exotic ants before release. Ant control measures could be taken, but in the past, chemical control of ants did more damage to the environment than to the targeted ant species (Summerlin et al. 1977; Williams et al. 2001; Oi et al. 2004; Tschinkel 2006; Plentovich et al. 2010). Future investigations will continue to document winners and losers as exotic and native species 
co-mingle in positive, neutral, and negative ways (Schoener et al. 2001; Allen et al. 2004;

King and Tschinkel 2006; Didham et al. 2007; Moreau et al. 2014; Lach et al. 2016).

Protecting pristine habitats and minimizing loss and degradation should be top priority to

maintain rare, specialist organisms sensitive to anthropogenic-mediated actions.

\section{REFERENCES}

Allen CR, Epperson DM, Garmestani AS (2004) Red imported fire ant impacts on wildlife: a decade of research. Am Midl Nat 152:88-103.

Andersen AN, Hoffmann BD, Muller WJ, Griffiths AD (2002) Using ants as bioindicators in land management: simplifying assessment of ant community responses. J Appl Ecol 39:8-17.

Bächtold A, Del-Claro K, Kaminski LA, Freitas AVL, Oliveira PS (2012) Natural history of an ant-plant-butterfly interaction in a Neotropical savanna. J Nat Hist 46:943-954.

Beuzelin JM, Reagan TE, Akbar W, Cormier HJ, Flanagan JW, Blouin DC (2009) Impact of Hurricane Rita storm surge on sugarcane borer (Lepidoptera: Crambidae) management in Louisiana. J Econ Entomol 102:1054-1061.

Brown WL (2000) Diversity of ants. In: Agosti D, Majer JD, Alonso LE, Schultz TR (eds) Ants: standard methods for measuring and monitoring biodiversity. Smithsonian Institution Press, Washington, DC, pp 45-79.

Buckley RC (1987) Interactions involving plants, Homoptera, and ants. Annu Rev Ecol Syst 18:111-135.

Buren WF, Allen GE, Whitcomb WH, Lennartz FE, Williams RN (1974) Zoogeography of the imported fire ants. J N Y Entomol Soc 82:113-124.

Calcaterra L, Cabrera S, Briano J (2016) Local co-occurrence of several highly invasive ants in their native range: are they all ecologically dominate species? Insectes Soc 63:407-419.

Carroll CR, Janzen DH (1973) Ecology of foraging by ants. Annu Rev Ecol Syst 4:231257. 
Castellanos I, Barbosa P, Zuria I, Caldas A (2015) Quantifying insect predation with predator exclusion cages: the role of prey antipredator behavior as a source of bias. Entomol Exp Appl 157:360-364.

Cuautle M, Rico-Gray V (2003) The effect of wasps and ants on the reproductive success of the extrafloral nectaried plant Turnera ulmifolia (Turneraceae). Funct Ecol $17: 417-423$.

Daniels JC (2014) Conservation matters: status and conservation of the federally endangered Schaus swallowtail butterfly. News of the Lepidopterists'. Society 56:138-139.

Dennis RL, Hodgson JG, Grenyer R, Shreeve TG, Roy DB (2004) Host plants and butterfly biology. Do host-plant strategies drive butterfly status? Ecol Entomol 29:12-26.

Deyrup M (2016) Ants of Florida: identification and natural history. CRC Press, Boca Raton.

Deyrup M, Davis L, Cover S (2000) Exotic ants in Florida. Trans Am Entomol Soc 126:293-326.

Didham RK, Tylianakis JM, Gemmell NJ, Rand TA, Ewers RM (2007) Interactive effects of habitat modification and species invasion on native species decline. Trends Ecol Evol 22:489-496.

Emmel TC, Minno MC, Nation JL Jr, Eliazar PJ, Cotter AJ, Bustamante EG, Ritland DB, Goode MR (1988) Habitat requirements and status of the endemic Schaus' swallowtail in the Florida Keys. Final Project Report, Project Number GFC-86-023. Submitted to Florida Game and Fresh Water Fish Commission Division of Wildlife Nongame Wildlife Section, Tallahassee, Florida.

Floren A, Biun A, Linsenmair KE (2002) Arboreal ants as key predators in tropical lowland rainforest trees. Oecologia 131:137-144.

Forys EA, Quistorff A, Allen CR (2001) Potential fire ant (Hymenoptera: Formicidae) impact on the endangered Schaus swallowtail (Lepidoptera: Papilionidae). Fla Entomol 84:254-258.

Forys EA, Allen CR, Wojcik DP (2002) Influence of the proximity and amount of human development and roads on the occurrence of the red imported fire ant in the lower Florida Keys. Biol Conserv 108:27-33.

Frankfater C, Tellez MR, Slattery M (2009) The scent of alarm: ontogenetic and genetic variation in the osmeterial gland chemistry of Papilio glaucus (Papilionidae) caterpillars. Chemoecology 19:81-96. 
Gentry GL, Dyer LA (2002) On the conditional, nature of neotropical caterpillar defenses against their natural enemies. Ecology 83:3108-3119.

Grieshop MJ, Werling B, Buehrer K, Perrone J, Isaacs R, Landis D (2012) Big brother is watching: studying insect predation in the age of digital surveillance. Am Entomol 58:172-182.

Heinrich B (1993) How avian predators constrain caterpillar foraging. In: Stamp NE, Casey TM (eds) Caterpillars ecological and evolutionary constraints on foraging. Chapman and Hall, Inc., New York, pp 224-247.

Holway DA, Lach L, Suarez AV, Tsutsui ND, Case TJ (2002) The causes and consequences of ant invasions. Annu Rev Ecol Syst 33:181-233.

Honda K (1983) Defensive potential of components of the larval osmeterial secretion of papilionid butterflies against ants. Physiol Entomol 8:173-179.

IBM SPSS Statistics 22 (2013) Statistical Package for the social sciences version 22. http://www.ibm.com/analytics/us/en/ technology/spss/.

Jameson A (2002) Host plants and habitats of the Schaus swallowtail butterfly (Papilio aristodemus ponceanus). MSc Thesis. University of Miami.

Jeanne RL (1979) A latitudinal gradient in rates of ant predation. Ecology 60:1211-1224.

Kaminski LA, Freitas AVL, Oliveira PS (2010) Interaction between mutualisms: anttended butterflies exploit enemy-free space provided by ant-treehopper associations. Am Nat 176:322-334.

King JR, Tschinkel WR (2006) Experimental evidence that the introduced fire ant, Solenopsis invicta, does not competitively suppress co-occurring ants in a disturbed habitat. J Anim Ecol 75:1370-1378.

Koptur S (1992) Plants with extrafloral nectaries and ants in Everglades habitats. Fla Entomol 75:38-50.

Koptur S, Rico-Gray V, Palacios-Rios M (1998) Ant protection of the nectaried fern Polypodium plebeium in central Mexico. Am J Bot 85:736-739.

Krushelnycky PD, Holway DA, Lebrun EG (2010) Invasion processes and causes of success. In: Lach L, Parr CL, Abbott KL (eds) Ant ecology. Oxford University Press, Oxford, pp 245-260.

Lach L, Hooper-Bùi LM (2010) Consequences of ant invasions. In: Lach L, Parr CL, Abbott KL (eds) Ant ecology. Oxford University Press, New York, pp 261-286. 
Lach L, Volp TM, Greenwood TA, Rose A (2016) High invasive ant activity drives predation of a native butterfly larva. Biotropica 48:146-149.

Loftus W, Kushlan J (1984) Population fluctuations of the Schaus swallowtail (Lepidoptera: Papilionidae) on the islands of Biscayne Bay, Florida, with comments on the Bahaman swallowtail. Fla Entomol 67:277-287.

Lopez R, Potter DA (2000) Ant predation on eggs and larvae of the black cutworm (Lepidoptera: Noctuidae) and Japanese beetle (Coleoptera: Scarabaeidae) in turfgrass. Environ Entomol 29:116-125.

Lowe S, Browne M, Boudjelas S, De Poorter M (2000) 100 of the world's worst invasive alien species: a selection from the global invasive species database. Invasive Species Specialist Group, Auckland.

Lubertazzi D, Tschinkel WR (2003) Ant community change across a ground vegetation gradient in north Florida's longleaf pine flatwoods. J Insect Sci 3:1-17.

MacGowan JC, Hill JG (2010) Two new exotic pest ants, Pseudomyrmex gracilis and Monomorium floricola (Hymenoptera: Formicidae) collected in Mississippi. Midsouth Entomol 3:106-109.

Mack MC, D’Antonio CM (1998) Impacts of biological invasions on disturbance regimes. Trends Ecol Evol 13:195-198.

Mathew G, Anto M (2007) In situ conservation of butterflies through establishment of butterfly gardens: a case study at Peechi, Kerala, India. Curr Sci 93:337-347.

McGlynn TP (1999) The worldwide transport of ants: geographic distribution and ecological invasions. J Biogeogr 26:535-548.

Mestre L, Piñol J, Barrientos JA, Espadaler X (2016) Differential ant exclusion from canopies shows contrasting top-down effects on community structure. Oecologia 180:190-203.

Minno MC (2015) 2015 Schaus' swallowtail (Heraclides aristodemus ponceanus) monitoring on Key Largo, Florida. South Lepidopterists News 37:106-107.

Minno MC, Emmel TC (1992) Larval protective coloration in swallowtails from the Florida Keys. Trop Lepidoptera 3:47-49.

Minno MC, Emmel TC (1993) Butterflies of the Florida Keys. Scientific Publishers, Gainesville. 
Montllor CB, Bernays EA (1993) Invertebrate predators and caterpillar foraging. In: Stamp NE, Casey TM (eds) Caterpillars ecological and evolutionary constraints on foraging. Chapman and Hall, Inc., New York, pp 170-202.

Morais HR, Diniz IR, Silva D (1999) Caterpillar seasonality in a central Brazilian Cerrado. Rev Biol Trop 47:1025-1033.

Moreau CS, Deyrup MA, Davis LR (2014) Ants of the Florida Keys: species accounts, biogeography, and conservation (Hymenoptera: Formicidae). J Insect Sci 14:295.

Nafus DM (1993) Movement of introduced biological-control agents onto nontarget butterflies, Hypolimnas spp. (Lepidoptera, Nymphalidae). Environ Entomol 22:265272.

Oi DH, Watson CA, Williams DF (2004) Monitoring and management of red imported fire ants in a tropical fish farm. Fla Entomol 87:522-527.

Oliveira PS, Freitas AVL (2004) Ant-plant-herbivore interactions in the neotropical cerrado savanna. Naturwissenschaften 91:557-570.

Oliveira PS, Oliveira-Filho AT, Cintra R (1987) Ant foraging on antinhabited Triplaris (Polygonaceae) in western Brazil: a field experiment using live termite-baits. J Trop Ecol 3:193-200.

Plentovich S, Swenson C, Reimer NJ, Richardson M, Garon N (2010) The effects hydramethylnon on the tropical fire ants (Solenopsis geminata) and non-target arthropods on Spit Island, Midway Atoll. J Insect Conserv 14:459-465.

Porter SD, Savignano DA (1990) Invasion of polygyne fire ants decimates native ants and disrupts arthropod community. Ecology 71:2095-2106.

Possley J, Hodges S, Magnaghi E, Maschinski J (2016) Distribution of Croton linearis in Miami-Dade County preserves with potential for supporting the federally endangered butterflies Strymon acis bartrami and Anaea troglodyta floridalis. Nat Area J 36:8187.

Reavey D (1993) Why body size matters to caterpillars. In: Stamp NE, Casey TM (eds) Caterpillars ecological and evolutionary constraints on foraging. Chapman and Hall, Inc., New York, pp 248-278.

Remmel T, Tammaru T, Magi M (2009) Seasonal mortality trends in tree-feeding insects: a field experiment. Ecol Entomol 34:98-106. 
Ribas CR, Schoereder JH, Pic M, Soares SM (2003) Tree heterogeneity, resource availability, and larger scale processes regulating arboreal ant species richness. Aust Ecol 28:305-314.

Saarinen EV, Daniels JC (2006) Miami blue butterfly larvae (Lepidoptera: Lycaenidae) and ants (Hymenoptera: Formicidae): new information on the symbionts of an endangered taxon. Fla Entomol 89:69-74.

Salazar JC, Whitman DC (2001) Defensive tactics of caterpillars against predators and parasitoids. In: Ananthakrishnan TN (ed) Insects and plant defence dynamics. Science Publishers, Inc., Plymouth, pp 161-207.

Salvato M (2008) Investigator's annual report to Biscayne National Park: Annual butterfly survey on Elliott Key for the North American Butterfly Association. Fish and Wildlife Service, Vero Beach, FL.

Sam K, Remmel T, Molleman F (2015) Material affects attack rates on dummy caterpillars in tropical forest where arthropod predators dominate: an experiment using clay and dough dummies with green colourants on various plant species. Entomol Exp Appl 157:317-324.

Schoener TW, Spiller DA, Losos JB (2001) Predators increase the risk of catastrophic extinction of prey populations. Nature 412:183-186.

Seifert CL, Lehner L, Adams M, Fiedler K (2015) Predation on artificial caterpillars is higher in countryside than near-natural forest habitat in lowland south-western Costa Rica. J Trop Ecol 31:281-284.

Seifert CL, Schulze CH, Dreschke CT, Frötscher H, Fiedler K (2016) Day vs. night predation on artificial caterpillars in primary rainforest habitats - an experimental approach. Entomol Exp Appl 158:54-59.

Sendoya SF, Oliveira PS (2015) Ant-caterpillar antagonism at the community level: Interhabitat variation of tritrophic interactions in a neotropical savanna. J Anim Ecol 84:442-452.

Sendoya SF, Blüthgen N, Tamashiro JY, Fernandez F, Oliveira PS (2016) Foliagedwelling ants in a neotropical savanna: effects of plant and insect exudates on ant communities. ArthropodPlant Interact 10:183-195.

Smiley JT (1985) Heliconius caterpillar mortality during establishment on plants with and without attending ants. Ecology 66:845-849. 
Snyder JR, Herndon A, Robertson WBJ (1990) South Florida Rockland. In: Myers RL, Ewel JJ (eds) Ecosystems of Florida. University Press of Florida, Gainesville, pp 230-277.

Stireman JO, Greeney HF, Dyer LA (2009) Species richness and host associations of Lepidoptera-attacking Tachinidae in the northeast Ecuadorian Andes. J Insect Sci 9:1-19.

Summerlin JC, Hung ACF, Vinson SB (1977) Residues in nontarget ants, species simplification and recovery of populations following aerial applications of mirex. Environ Entomol 6:193-197.

Tilman D (1978) Cherries, ants and tent caterpillars: timing of nectar production in relation to susceptibility of caterpillars to ant predation. Ecology 59:686-692.

Timms LL, Schwarzfeld M, Saaksjarvi IE (2016) Extending understanding of latitudinal patterns in parasitoid wasp diversity. Insect Conserv Divers 9:74-86.

Tobin JE (1995) Ecology and diversity of tropical forest canopy ants. In: Lowman MD, Nadkarni NM (eds) Forest canopies. Academic Press, New York, pp 129-147.

Tschinkel WR (1988) Distribution of the fire ants Solenopsis invicta and S. geminata (Hymenoptera: Formicidae) in northern Florida in relation to habitat and disturbance. Ann Entomol Soc Am 81:76-81.

Tschinkel WR (2006) The fire ants. The Belknap Press of Harvard University Press, Massachusetts.

United States Fish and Wildlife Service (FWS) (2008) Schaus swallowtail butterfly (Heraclides aristodemus ponceanus) 5-Year review: Summary and evaluation. http://ecos.fws.gov/docs/five_year_review/doc1983.pdf. Accessed 11 Nov 2016.

Vitousek PM, D’Antonio CM, Loope LL, Westbrooks R (1996) Biological invasions as global environmental change. Am Sci 84:468-478.

Wagner DL, Van Driesche RG (2010) Threats posed to rare and endangered insects by invasions of nonnative species. Annu Rev Entomol 55:547-568.

Wang CL, Strazanac J, Butler L (2001) A comparison of pitfall traps for studying leaf litter ant communities. J Econ Entomol 94:761-765.

Watanabe M (1981) Population dynamics of the swallowtail butterfly, Papilio xuthus L., in a deforested area. Res. Popul Ecol 23:74-93. 
Weseloh RM (1993) Potential effects of parasitoids on the evolution of caterpillar foraging behavior. In: Stamp NE, Casey TM (eds) Caterpillars ecological and evolutionary constraints on foraging. Chapman and Hall, Inc., New York, pp 203223.

Wetterer JK (2010) Worldwide spread of the graceful twig ant, Pseudomyrmex gracilis (Hymenoptera: Formicidae). Fla Entomol 93:535-540.

Wetterer JK, Porter SD (2003) The little fire ant, Wasmannia auropunctata: distribution, impact, and control. Sociobiology 42:1-41.

Whelan KRT (2011) Schaus swallowtail butterfly (Heraclides aristodemus ponceanus) habitat enhancement in Biscayne National Park. Project Report. Funded by FWS South Florida Coastal Program. National Park Service, Palmetto Bay, Florida

Whelan KRT, Atkinson A (2015) Schaus Swallowtail Butterfly (Heraclides aristodemus ponceanus) Habitat Enhancement in Biscayne National Park. South Florida Coastal Program Project Progress Report. NPS/SFCN. National Park Service, Palmetto Bay, Florida.

Whelan KRT, Ruiz PL, Shamblin RB, Houle PA, Ross MS, Atkinson AJ, Patterson JM, Alonso J (2013) Biscayne National Park Vegetation Map Project. Natural Resource Technical Report. NPS/SFCN/NRTR-2013/774. National Park Service, Fort Collins, Colorado.

Whitcomb WH, Denmark HA, Buren WF, Carroll JF (1972) Habits and present distribution in Florida of the exotic ant, Pseudomyrmex mexicanus (Hymenoptera: Formicidae). Fla Entomol 55:31-33.

Williams DF (1994) Biology, impact, and control of introduced species. Westview Press, Boulder.

Williams DF, Collins HL, Oi DH (2001) The red imported fire ant (Hymenoptera: Formicidae): an historical perspective of treatment programs and the development of chemical baits for control. Am Entomol 47:146-159.

Zettler JA, Taylor MD, Allen CR, Spira TP (2004) Consequences of forest clear-cuts for native and non-indigenous ants (Hymenoptera: Formicidae). Ann Entomol Soc Am 97:513-518. 


\section{TABLES}

Table 3.1: Ants collected in pitfall traps at Elliott and Adams Keys: number of individuals of each species, and status. Ant species status in south Florida determined by several references (Moreau et al. 2014; Deyrup 2016).

\begin{tabular}{|l|l|l|l|l|}
\hline & \multicolumn{1}{|c|}{ Subfamily } & \multicolumn{1}{|c|}{ Species } & Number & Native or Exotic \\
\hline 1 & Formicinae & Camponotus floridanus & 314 & Native \\
\hline 2 & Formicinae & Camponotus planatus & 241 & Exotic \\
\hline 3 & Formicinae & Camponotus tortuganus & 7 & Native (Probably) \\
\hline 4 & Formicinae & Nylanderia steinheili & 185 & Exotic \\
\hline 5 & Formicinae & Brachymyrmex depilis & 12 & Native \\
\hline 6 & Formicinae & Brachymyrmex obscurior & 10 & Native (Probably) \\
\hline 7 & Pseudomyrmecinae & Pseudomyrmex gracilis & 215 & Exotic \\
\hline 8 & Pseudomyrmecinae & Pseudomyrmex elongatus & 12 & Exotic (Probably) \\
\hline 9 & Pseudomyrmecinae & Pseudomyrmex ejectus & 3 & Native \\
\hline 10 & Pseudomyrmecinae & Pseudomyrmex simplex & 1 & Native (Probably) \\
\hline 11 & Myrmicinae & Crematogaster ashmeadi & 147 & Native \\
\hline 12 & Myrmicinae & Pheidole dentata & 90 & Native \\
\hline 13 & Myrmicinae & Pheidole floridana & 11 & Native \\
\hline 14 & Myrmicinae & Pheidole moerens & 2 & Exotic \\
\hline 15 & Myrmicinae & Monomorium floricola & 52 & Exotic \\
\hline 16 & Myrmicinae & Temnothorax allardycei & 30 & Native (Probably) \\
\hline 17 & Myrmicinae & Aphaenogaster miamiana & 24 & Native \\
\hline 18 & Myrmicinae & Cyphomyrmex minutus & 16 & Native (Probably) \\
\hline 19 & Myrmicinae & Solenopsis invicta & 15 & Exotic \\
\hline 20 & Myrmicinae & Solenopsis geminata & 13 & Native \\
\hline 21 & Myrmicinae & Cyphomyrmex minutior & 3 & Exotic \\
\hline 22 & Myrmicinae & Wasmannia auropunctata & 2 & Exotic \\
\hline 23 & Dolichoderinae & Tapinoma melanocephalum & 8 & Exotic \\
\hline 24 & Ponerinae & Odontomachus brunneus & 4 & Native \\
\hline 25 & Ponerinae & Odontomachus ruginodis & 1 & Exotic (Probably) \\
\hline
\end{tabular}


Table 3.2: Ants collected in pitfall traps in the canopies of Amyris elemifera and

Zanthoxylum fagara: number of individuals of each species, and status. Ant species status in south Florida determined by several references (Moreau et al. 2014; Deyrup 2016).

\begin{tabular}{|l|l|l|l|l|}
\hline & \multicolumn{1}{|c|}{ Subfamily } & \multicolumn{1}{|c|}{ Species } & Number & Native or Exotic \\
\hline 1 & Formicinae & Camponotus floridanus & 32 & Native \\
\hline 2 & Formicinae & Camponotus planatus & 51 & Exotic \\
\hline 3 & Formicinae & Camponotus tortuganus & 2 & Native (Probably) \\
\hline 4 & Formicinae & Brachymyrmex depilis & 8 & Native \\
\hline 5 & Formicinae & Brachymyrmex obscurior & 3 & Native (Probably) \\
\hline 6 & Pseudomyrmecinae & Pseudomyrmex gracilis & 84 & Exotic \\
\hline 7 & Pseudomyrmecinae & Pseudomyrmex elongatus & 3 & Exotic (Probably) \\
\hline 8 & Pseudomyrmecinae & Pseudomyrmex ejectus & 1 & Native \\
\hline 9 & Myrmicinae & Crematogaster ashmeadi & 45 & Native \\
\hline 10 & Myrmicinae & Monomorium floricola & 3 & Exotic \\
\hline 11 & Myrmicinae & Temnothorax allardycei & 8 & Native (Probably) \\
\hline 12 & Dolichoderinae & Tapinoma melanocephalum & 3 & Exotic \\
\hline
\end{tabular}


Table 3.3: Generalized linear model (negative binomial with log link) results of Pseudomyrmex gracilis collected on three different groups of trees.

\begin{tabular}{|l|c|c|c|}
\hline \multicolumn{1}{|c|}{ Parameter } & Mean & SD & SE \\
\hline Amyris elemifera & 1.590 & 2.080 & 0.256 \\
\hline Zanthoxylum fagara & 1.450 & 1.927 & 0.346 \\
\hline Non-Host Tree & 0.990 & 1.421 & 0.170 \\
\hline \multicolumn{4}{|c|}{ Omnibus Test } \\
\hline \multicolumn{3}{|c|}{ df } \\
\hline \multicolumn{4}{|c|}{2} \\
\hline \multicolumn{4}{|c|}{ Hypothesis Test } \\
\hline \multicolumn{1}{|c|}{ Parameter } & Wald Chi-Square & $\boldsymbol{d f}$ & $\boldsymbol{p}$-value \\
\hline Intercept & 8.357 & 1 & 0.004 \\
\hline Amyris elemifera & 4.119 & 1 & 0.042 \\
\hline Zanthoxylum fagara & 0.102 & 1 & 0.750 \\
\hline Non-Host Tree & - & - & - \\
\hline
\end{tabular}


Table 3.4: Results of predator exclusion experiment using H. cresphontes larvae on Amyris elemifera and Zanthoxylum fagara. Z scores (adjusted z-scores) were acquired through the contingency table analysis and then squared to obtain Chi square values. Chi square values were used to calculate exact p-values with the significance function group in SPSS. Exact $\mathrm{p}$ values were compared to the adjusted $\alpha$-value to determine significance. The adjusted $\alpha$-value based on the Bonferroni correction was set at 0.00416 $(0.05 / 12)$. Exact $p$ values equal to or less than the adjusted $\alpha$-value were considered significant. Asterisk denotes significance.

Treatments defined in first column.

\begin{tabular}{|l|l|l|l|l|}
\hline \multicolumn{1}{|c|}{ Treatments } & \#Alive/Total \# of Trials & Adjusted z-score & Chi square & $p$ value \\
\hline Early instar caterpillars & & & & \\
\hline 1) no barriers (no tanglefoot/no cage) & $3 / 16=18.7 \%$ & 4.70 & 22.09 & $0.000003^{*}$ \\
\hline 2) tanglefoot present/no cage & $5 / 16=31.3 \%$ & 3.50 & 12.25 & $0.000465^{*}$ \\
\hline 3) no tanglefoot/cage with holes present & $6 / 16=37.5 \%$ & 3.00 & 9.00 & $0.002700^{*}$ \\
\hline 4) tanglefoot present/cage with holes present & $14 / 16=87.5 \%$ & -1.60 & 2.56 & 0.109599 \\
\hline 5) no tanglefoot/cage present & $14 / 16=87.5 \%$ & -1.60 & 2.56 & 0.109599 \\
\hline 6) tanglefoot present/cage present & $15 / 16=93.8 \%$ & -2.20 & 4.84 & 0.027807 \\
\hline Late instar caterpillars & & & & \\
\hline 1) no barriers (no tanglefoot/no cage) & $5 / 15=33.3 \%$ & 3.20 & 10.24 & $0.001374 *$ \\
\hline 2) tanglefoot present/no cage & $11 / 15=73.3 \%$ & -0.30 & 0.09 & 0.764177 \\
\hline 3) no tanglefoot/cage with holes present & $13 / 15=86.7 \%$ & -1.50 & 2.25 & 0.133614 \\
\hline 4) tanglefoot present/cage with holes present & $15 / 15=100 \%$ & -2.70 & 7.29 & 0.006934 \\
\hline 5) no tanglefoot/cage present & $14 / 15=93.3 \%$ & -2.10 & 4.41 & 0.035729 \\
\hline 6) tanglefoot present/cage present & $15 / 15=100 \%$ & -2.70 & 7.29 & 0.006934 \\
\hline
\end{tabular}


Table 3.5: Results from the ant-caterpillar interaction study. Four common ant species interacted with early and late instar caterpillars placed on Amyris elemifera and Zanthoxylum fagara. Total mortality based on interactions with ants included larvae (caterpillar) removal or leaf abortion. Major damage was caused by Pseudomyrmex gracilis (early instar mortality was $86.7 \%$ ) and Camponotus floridanus (early instar mortality was $56.3 \%$, late instar mortality was $40 \%$ ).

\begin{tabular}{|l|l|l|l|l|l|l|}
\hline \multicolumn{1}{|c|}{ Ant Species } & \multicolumn{3}{c|}{ Early Instars } & \multicolumn{1}{c|}{ Late Instars } \\
\hline & $\begin{array}{l}\text { Removed } \\
\text { By Ant(s) }\end{array}$ & $\begin{array}{l}\text { Aborted } \\
\text { Leaf }\end{array}$ & $\begin{array}{l}\text { Mortality } \\
\text { (removed+aborted) } \\
\text { \# of trials }\end{array}$ & $\begin{array}{l}\text { Removed } \\
\text { By Ant(s) }\end{array}$ & $\begin{array}{l}\text { Aborted } \\
\text { Leaf }\end{array}$ & $\begin{array}{c}\text { Mortality } \\
\text { (removed+aborted) }\end{array}$ \\
\hline Camponotus planatus & 0 & 0 & $0 / 16=0 \%$ & 0 & 0 & $0 / 15=0 \%$ \\
\hline Crematogaster ashmeadi & 1 & 1 & $2 / 10=20 \%$ & 0 & 0 & $0 / 11=0 \%$ \\
\hline Camponotus floridanus & 7 & 2 & $9 / 16=56.3 \%$ & 0 & 6 & $6 / 15=40 \%$ \\
\hline Pseudomyrmex gracilis & 11 & 2 & $13 / 15=86.7 \%$ & 0 & 0 & $0 / 15=0 \%$ \\
\hline
\end{tabular}


Table 3.6: Ant Danger Index (ADI) based on cumulative scores from: (1) ant species relative abundance, (2) time to discover caterpillar, (3) ability to recruit more ants, (4) early instar (small) mortality, and (5) late instar (large) mortality. The total score for each ant species was divided by 25 (max score each ant species could acquire) and then multiplied by 100 for a final max score.

The final max score with a range 0-100 ranked each ant species against the other three ant species. All interactions between native and exotic ants and caterpillars occurred on Amyris elemifera and Zanthoxylum fagara in the forests at Elliott and Adams Keys.

\begin{tabular}{|c|c|c|c|c|c|c|c|}
\hline Ant Species & Origin & $\begin{array}{c}\text { 1) } \\
\text { Abundance }\end{array}$ & $\begin{array}{c}\text { 2) } \\
\text { Discovery }\end{array}$ & $\begin{array}{c}\text { 3) } \\
\text { Recruit }\end{array}$ & $\begin{array}{l}\text { 4) } \\
\text { Mortality } \\
\text { (Small) }\end{array}$ & $\begin{array}{c}\text { 5) } \\
\text { Mortality } \\
\text { (Large) }\end{array}$ & $\begin{array}{c}\text { ADI } \\
\text { Total score } \\
\text { (max: 100) }\end{array}$ \\
\hline $\begin{array}{l}\text { Scale used for each category } \\
\text { (how the values } 0-5 \text { were } \\
\text { assigned) }\end{array}$ & & $\begin{array}{l}\text { 0) } 0 \% \\
\text { 1) } 1-10 \% \\
\text { 2) } 11-20 \% \\
\text { 3) } 21-30 \% \\
\text { 4) } 31-40 \% \\
\text { 5) }>40 \%\end{array}$ & $\begin{array}{l}\text { 0) }>300 \mathrm{~s} \\
\text { 1) } 181-300 \mathrm{~s} \\
\text { 2) } 121-180 \mathrm{~s} \\
\text { 3) } 61-120 \mathrm{~s} \\
\text { 4) } 11-60 \mathrm{~s} \\
\text { 5) } 0-10 \mathrm{~s}\end{array}$ & $\begin{array}{l}\text { 0) } 0 \text { ants } \\
\text { 1) } 1 \text { ant } \\
\text { 2) } 2-3 \text { ants } \\
\text { 3) } 4-6 \text { ants } \\
\text { 4) } 7-10 \text { ants } \\
\text { 5) }>10 \text { ants }\end{array}$ & $\begin{array}{l}\text { 0) } 0 \% \\
\text { 1) } 1-20 \% \\
\text { 2) } 21-40 \% \\
\text { 3) } 41-60 \% \\
\text { 4) } 61-80 \% \\
\text { 5) } 81-100 \%\end{array}$ & $\begin{array}{l}\text { 0) } 0 \% \\
\text { 1) } 1-20 \% \\
\text { 2) } 21-40 \% \\
\text { 3) } 41-60 \% \\
\text { 4) } 61-80 \% \\
\text { 5) } 81-100 \%\end{array}$ & \\
\hline A) Crematogaster ashmeadi & Native & 2 & 2 & 1 & 1 & 0 & 24 \\
\hline B) Camponotus planatus & Exotic & 3 & 3 & 0 & 0 & 0 & 24 \\
\hline C) Camponotus floridanus & Native & 2 & 2 & 1 & 3 & 2 & 40 \\
\hline D) Pseudomyrmex gracilis & Exotic & 4 & 4 & 0 & 5 & 0 & 52 \\
\hline
\end{tabular}




\section{FIGURES}

Figure 3.1: Geographic range of the federally endangered Heraclides aristodemus ponceanus in south Florida. The historic ranged is outlined in yellow, the current range is outlined in red, and the boundary of Biscayne National Park is outlined in blue. Map generated by Lydia Cuni.

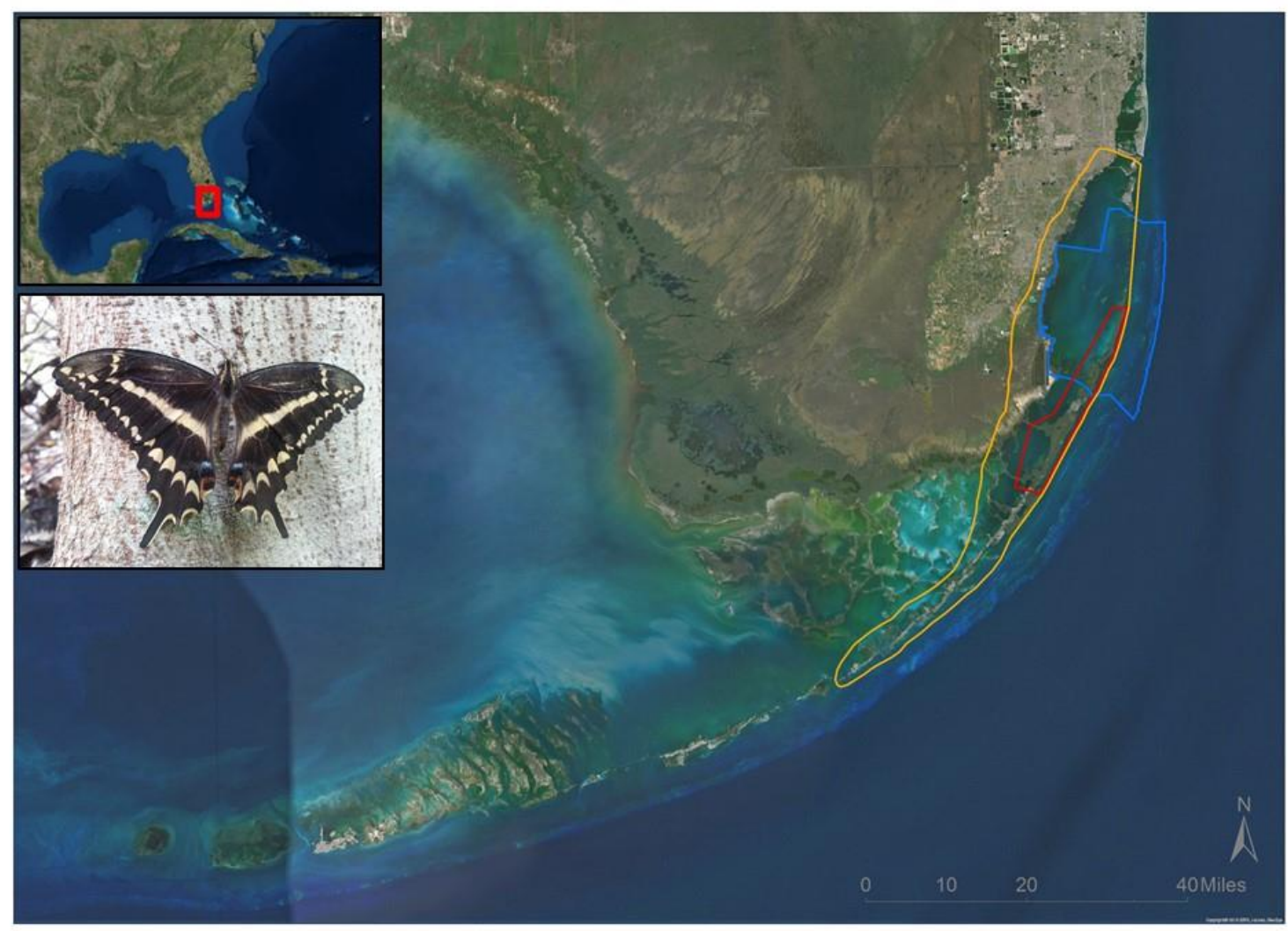


Figure 3.2: Percentage of ant species captured in pitfall traps at Elliott and Adams Keys (tree canopy, trunk, and base). Overall, 1418 total ants comprising 25 ant species were captured and identified from pitfall traps.

\section{Total Ants Surveyed on Elliott and Adams Keys}

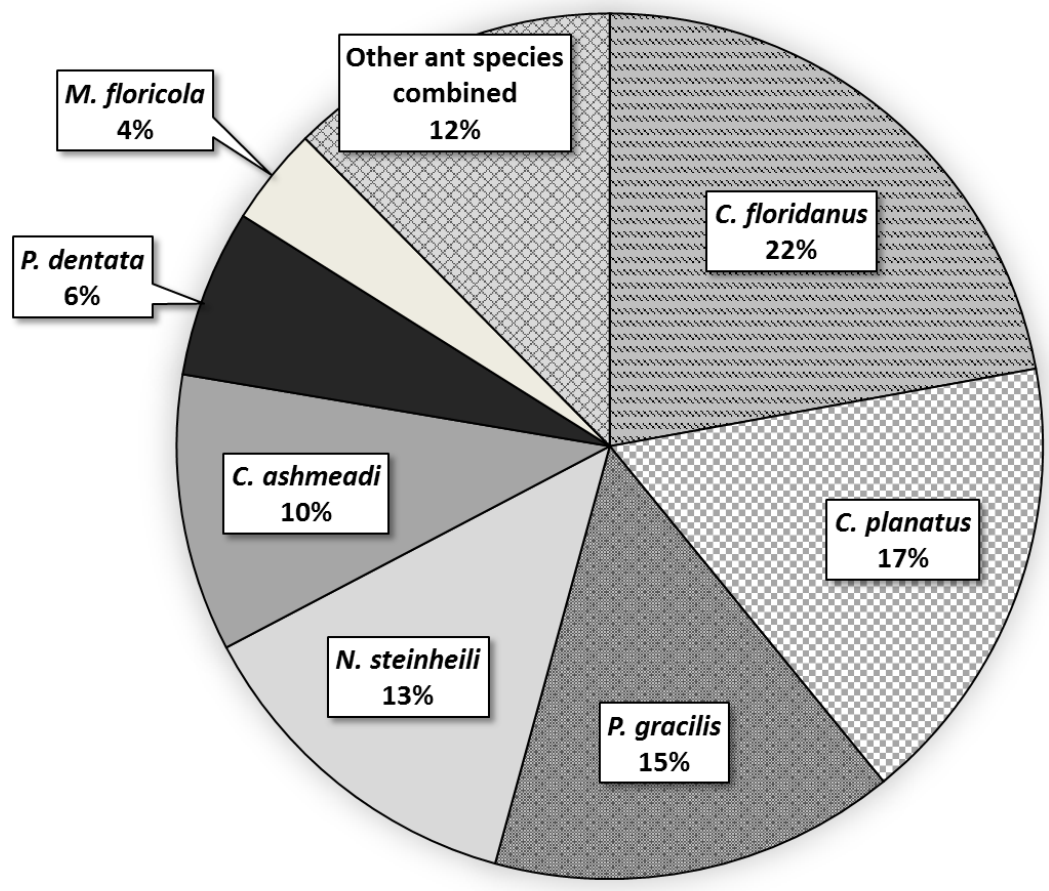


Figure 3.3: Percentage of ant species captured in pitfall traps on Amyris elemifera and Zanthoxylum fagara. Overall, 243 total ants comprising 12 ant species were captured and identified from pitfall traps used in the canopy. The four most common ants collected were $P$. gracilis, $C$. planatus, $C$. ashmeadi, and $C$. floridanus. They represented $87 \%$ of all collected ants on A. elemifera and Z. fagara. Pseudomyrmex gracilis and C. planatus are exotic species and represented $56 \%$ of the total sampled ants.

\section{Total Ants Surveyed in the Tree Canopy (Amyris elemifera and Zanthoxylum fagara)}

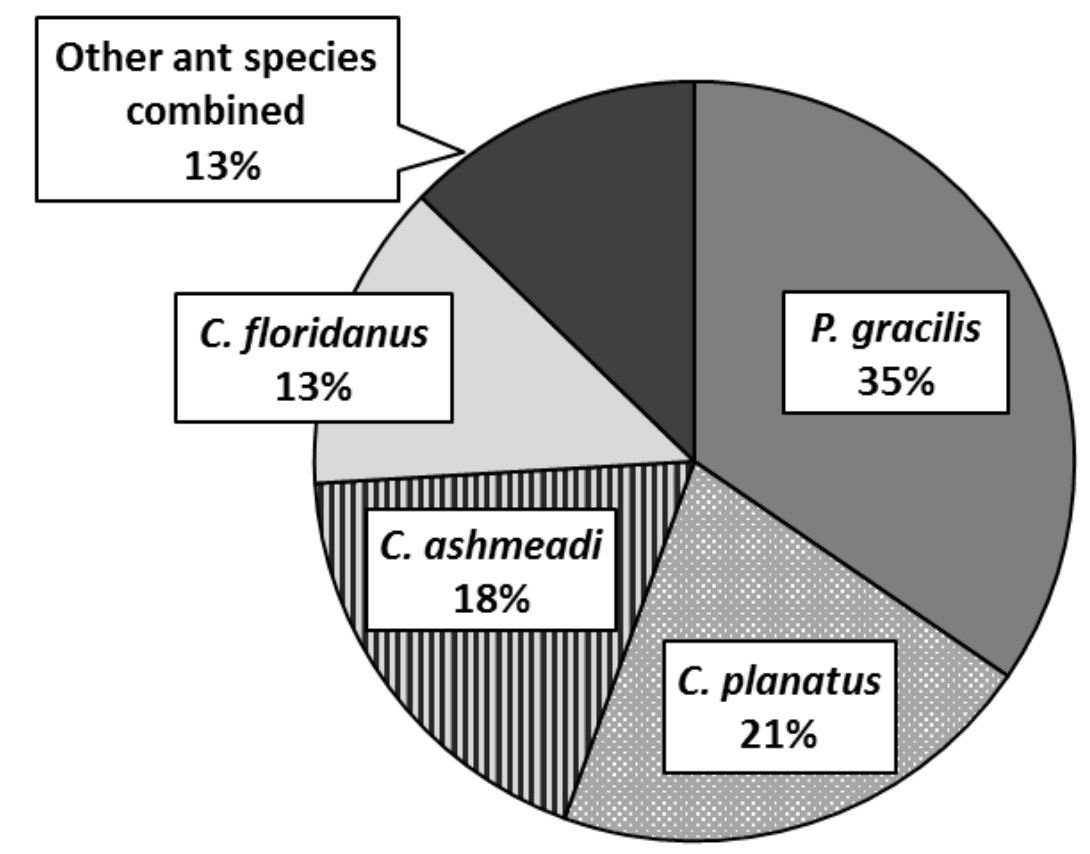


Figure 3.4: Graphical representation of Pseudomyrmex gracilis collected on three different group of trees at Elliott and Adams Keys in Biscayne National Park, Florida. The bars represent averages of count data with standard error bars. Lowercase letters reflect statistical significance. Overall, $P$. gracilis was only significantly more frequent on Amyris elemifera than non-host trees. Amyris elemifera is the primary host tree for the Heraclides aristodemus ponceanus.

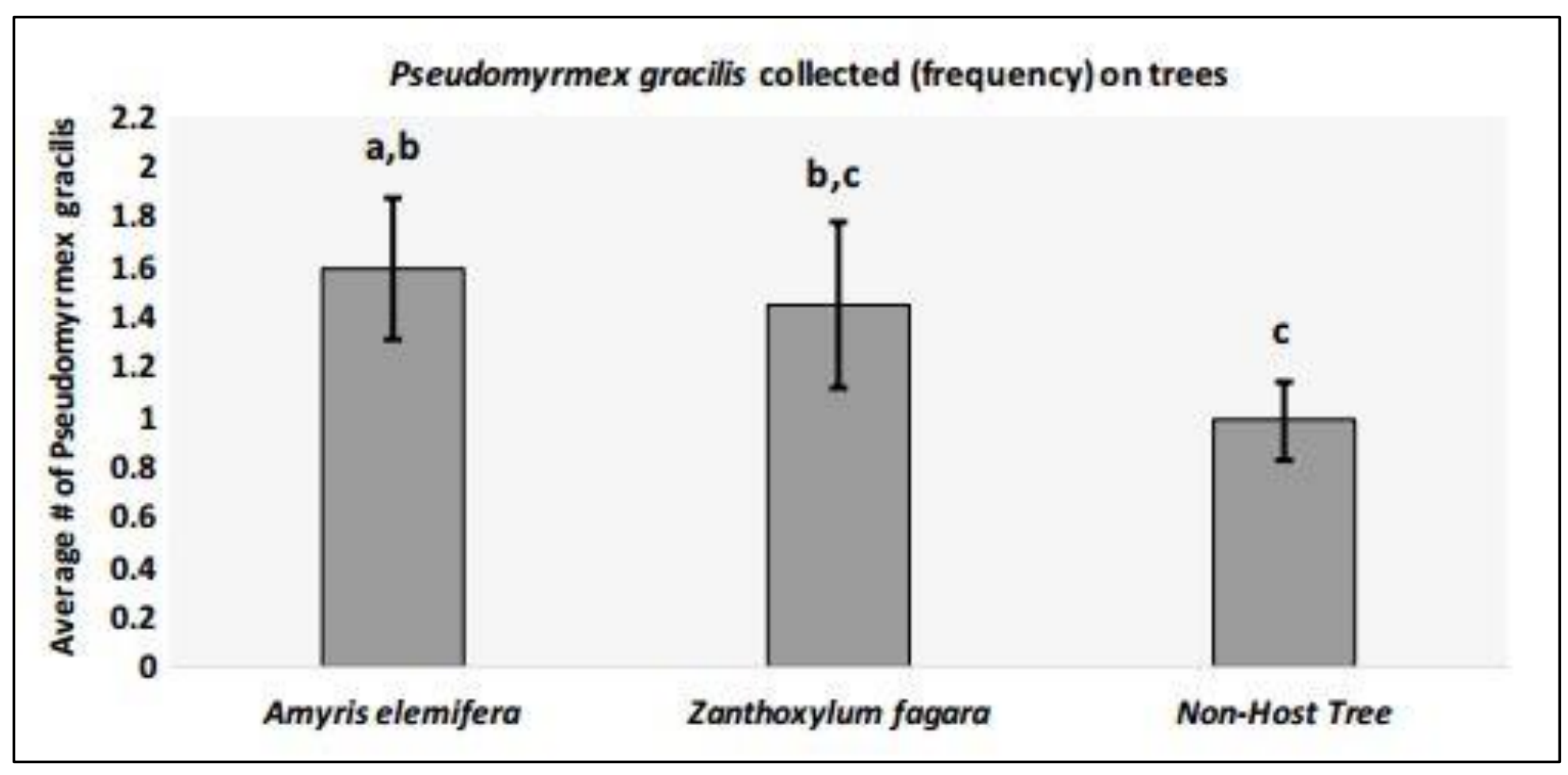


Figure 3.5: The predator exclusion experiment involved 6 treatments, with physical barriers (tanglefoot and mesh cages) used in different combinations on Zanthoxylum fagara to compare caterpillar (early and late instar) survivorship against crawling and non-crawling predators. Survivorship was defined as persistence on leaf for 5 days. A contingency table analysis was performed. Asterisks denote significance in survivorship for the various treatments. Each treatment using early instar caterpillars had 16 trials, each treatment using late instar caterpillars had 15 trials. Refer to Table 4 for more details.

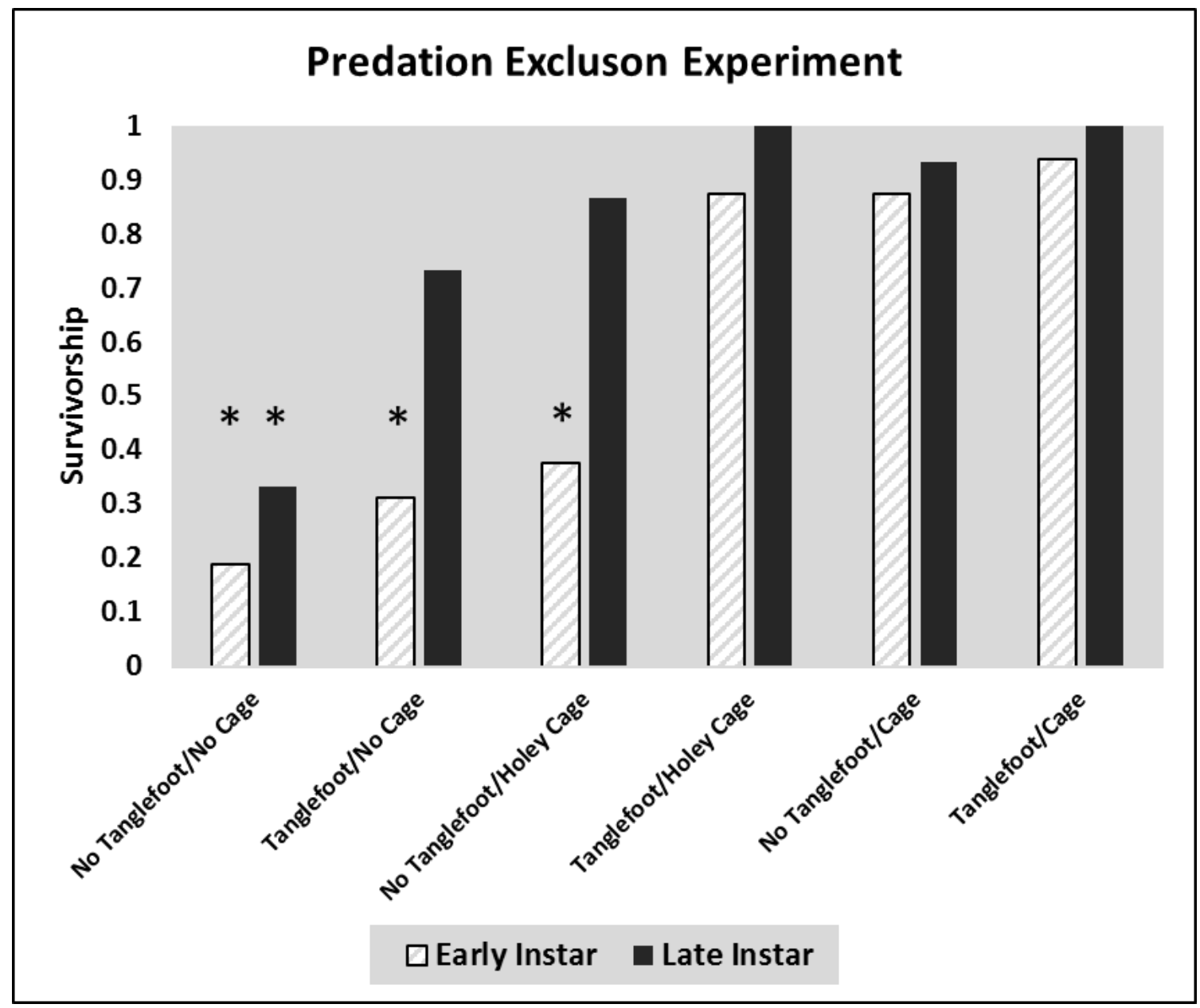


Figure 3.6: The average time $( \pm \mathrm{SE})$ the four most abundant ant species took to discover Heraclides cresphontes caterpillars placed on Amyris elemifera and Zanthoxylum fagara at Elliott Key, Biscayne National Park, Florida. A one-way ANOVA was performed followed by the Fisher's Least Significant Difference post-hoc test. Asterisk denotes significance.

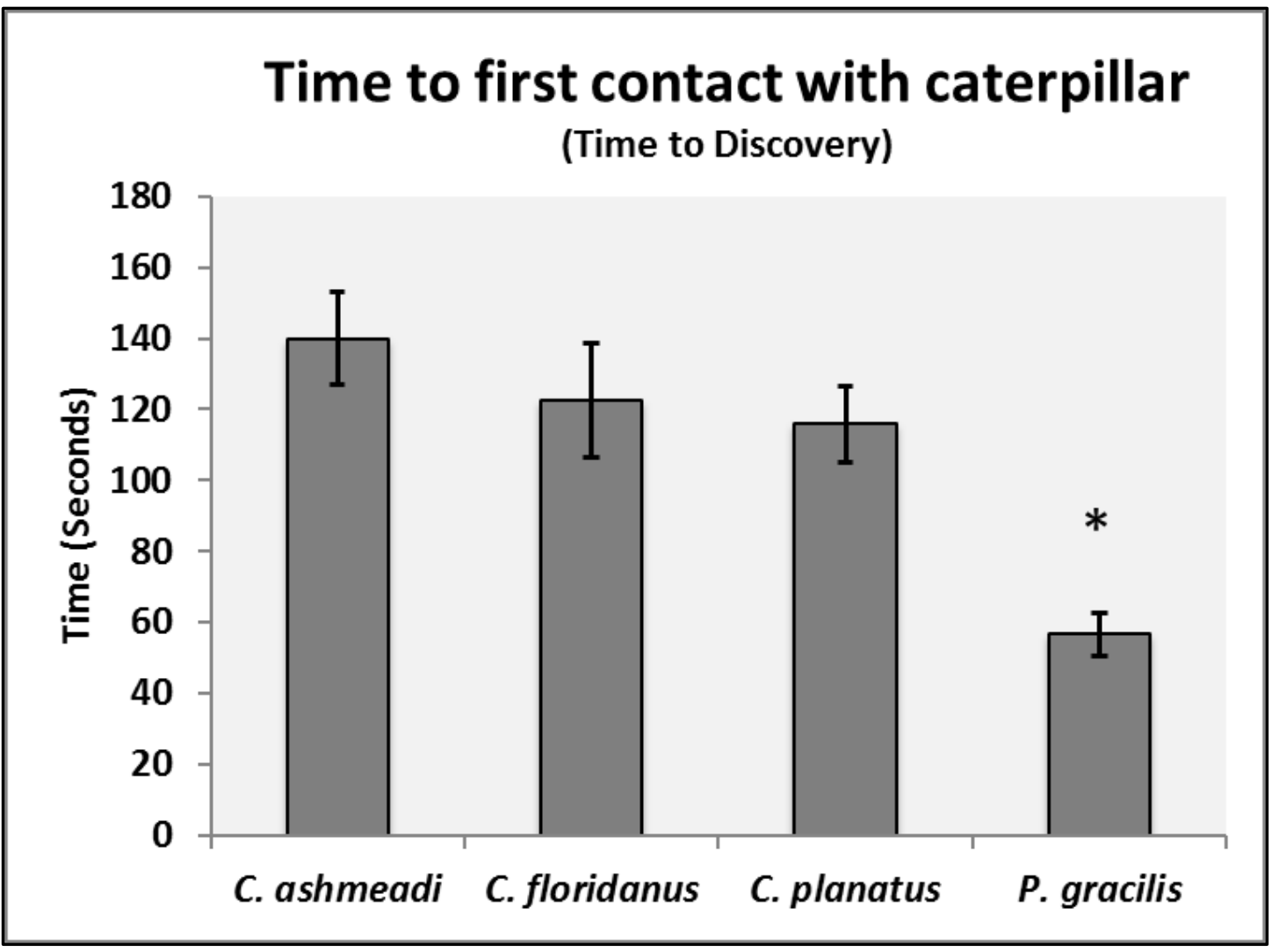


Figure 3.7: Ant recruitment to Heraclides cresphontes caterpillars placed on Amyris elemifera and Zanthoxylum fagara by the four most abundant ant species at Elliott Key, Biscayne National Park, Florida. Recruitment is displayed as the average number of ants attracted to caterpillars $( \pm$ SE). Ant recruitment allows ants to overwhelm larger prey such as late instar caterpillars.

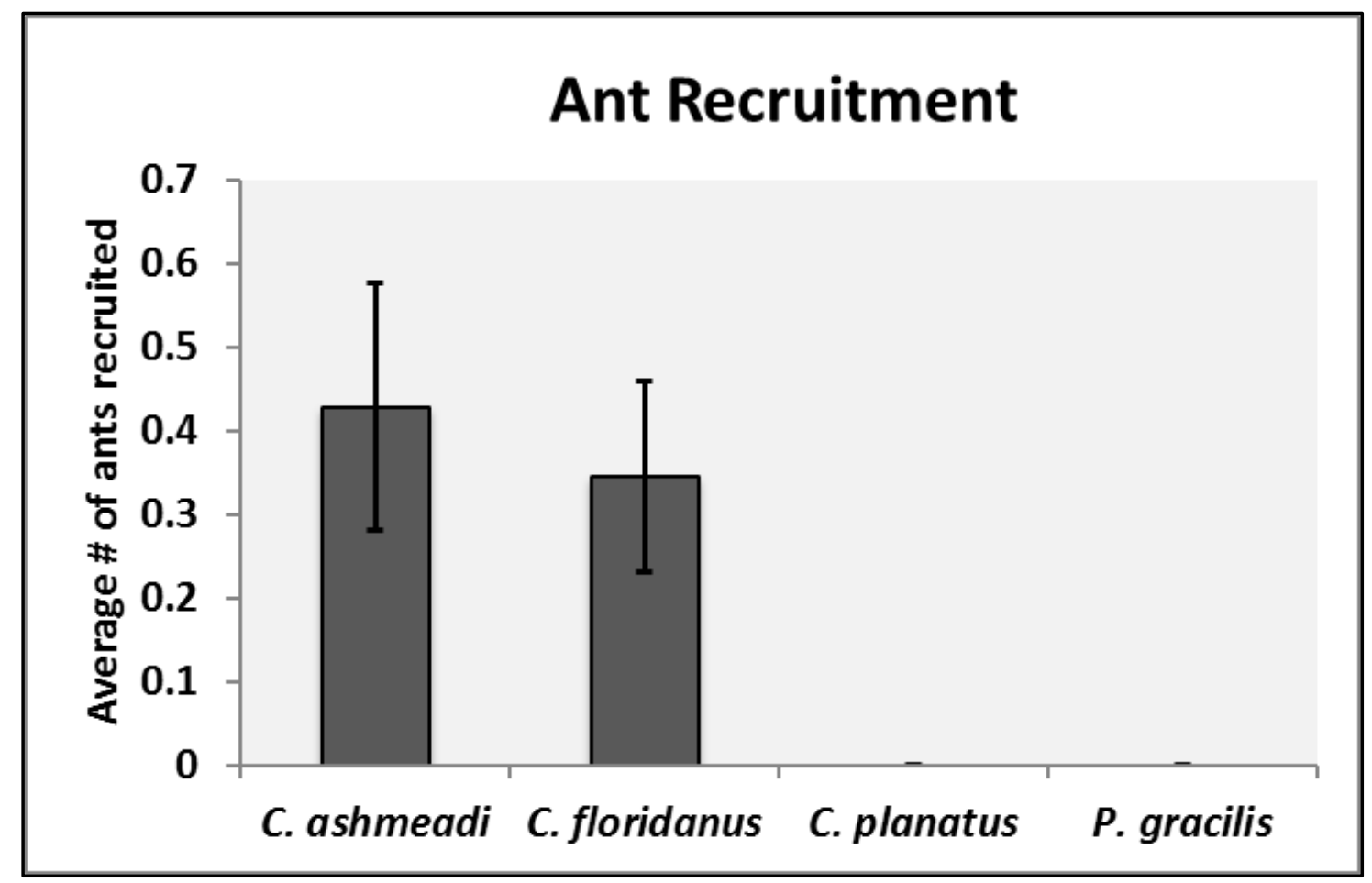


Figure 3.8: Early instar Heraclides aristodemus ponceanus caterpillar fends off an approach by Camponotus planatus. This approach is not aggressive; however, the H. $a$. ponceanus caterpillar does not tolerate contact by other organisms.

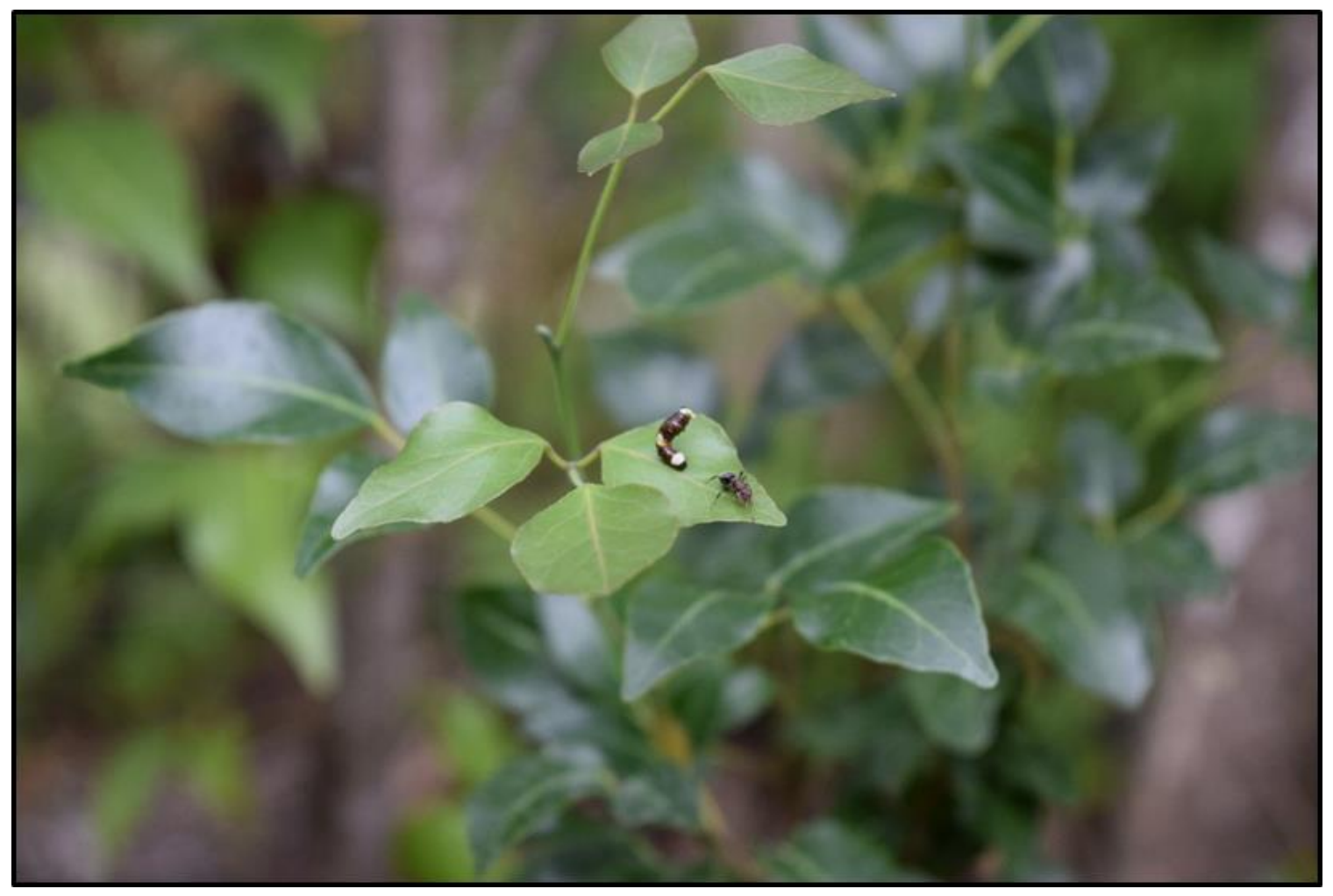




\section{CHAPTER IV}

BUTTERFLY GARDENING AT MIAMI URBAN SCHOOLS: PLUGGING STUDENTS INTO NATURE THROUGH HABITAT REHABILITATION ON SCHOOL GROUNDS 


\section{ACKNOWLEDGEMENTS}

We thank the principals at each school: Tamela Brown (Whispering Pines Elementary), Yanelys Canales (North Hialeah Elementary), and Raul Calzadilla, Jr. (Air Base K-8 Center). The study would not have been feasible without the dedication and support of the teachers involved: Sarah Davio (Whispering Pines Elementary), Nadia Earl (North Hialeah Elementary), Jessica Hernandez (North Hialeah Elementary), Sandra Martinez (Air Base K-8 Center), Carmen Monge (Air Base K-8 Center), Kamelia Ramnarine (Whispering Pines Elementary), Vicki Terranova (Whispering Pines Elementary), Louis Vazquez (North Hialeah Elementary). We thank Dr. Scott Zona for provided FIU greenhouse space and materials. We also thank Leslie Bayas, Lydia Cuni, Darlene Darrican, Michael Foguer, Tanjim Hossain, Eduardo Ibarra, Craig Perry, Andrea Salas, and Yadira Reynaldo for leadership roles involved in garden construction, plant care and transportation. Jennifer Morales provided invaluable consultation on the instruments (tests, survey statements, and interview questions) designed for this study. George O'Brien and Sandra Martinez reviewed the interview transcripts with pseudonyms for coherent themes. Constructive comments on the manuscript including statistical support were made by Sarah Eddy.

This study was financially supported through FIU Center for Leadership Global Civic Engagement Mini Grant, Kelly Foundation Botany Scholarship, and DonorsChoose.org (raised contributions for supplies at North Hialeah and Whispering Pines Elementary schools). Many butterfly host and nectar plants donated to the students and schools were provided by Mark Glasser, whose compassion and advocacy for placed-based education, insect conservation, and civic engagement helped fuel the study as many plants were 
needed and stored until further use at the schools. Additional plants were provided by members in the North American Butterfly Association Miami Blue Chapter and Florida Native Plant Society Tillandsia Chapter. Permission to conduct research activities with human subjects was granted through the Institutional Review Board \# IRB-15-0080. Constructive comments were provided by Adel Peña, Maureen Donnelly, Bradley Bennet, and Jeffrey Wells. This is contribution \#_ to the Tropical Biology Program at Florida International University.

\begin{abstract}
Habitat loss is a major factor that contributes to the decline of butterflies and other insects globally. School grounds provide an opportunity to restore green space in the local community. Three schools in Miami-Dade County participated in a south Florida native plant butterfly garden study. The "Schaus and Coastal Hardwood Hammock" curriculum unit, containing multiple sequenced lesson plans, focused on active-learning and collaboration within the school community. At each school, students designed, constructed, and cared for native plant butterfly gardens (with an emphasis on imperiled butterfly species).

Two fifth grade classes from three schools were separated into experimental and control groups for a total of 3 experimental groups and 3 control groups. During this 3month study, both groups at each school were administered pre- and post-tests, surveys, and interviews. Each student in the experimental group received butterfly and wildflower identification guides, as well as host and nectar plants to take home; they maintained
\end{abstract}


plants and recorded insect activity for two months. Control group students did not receive any take-home items.

Collectively, students in the experimental group scored significantly higher on the post-test than the pre-test, in contrast to no significant increase in the control group. In addition, an increase in post-test scores for the host and nectar plants intervention (experimental group) was significant for all three schools. Pre- and post-surveys revealed students demonstrated favorable interest in animals and plants and were advocates for environmental stewardship; however, they reported less interest in insects. The interviews revealed most students associated outdoor class time with physical education (structured play time) at school. If students had gardens at home, they mainly consisted of edible and ornamental plants. All interviewed students stated, "They would recommend a butterfly garden to a friend" during post-interviews. After project completion, more students in the experimental group had new gardens than students in the control group. The study demonstrates the tangible effect of outdoor schoolyard learning activities in student knowledge of conservation issues, and the importance of hands-on experience in engaging students to advocate for butterflies.

Key words: Active-learning, butterfly gardens, insect conservation, native plants, outdoor classroom, place-based education 


\section{INTRODUCTION}

Butterfly species decline because of habitat loss

We are in a distinctive chapter in history: scientists have suggested we have transitioned from the Holocene epoch to the Anthropocene epoch (Crutzen, 2002; Lewis and Maslin, 2015; Hazen et al., 2017). The Anthropocene represents the current chapter in human history where we have a profound impact on Earth's systems and living species (Crutzen, 2002; Dirzo et al., 2014; Lewis and Maslin, 2015). Humans have eliminated and severely reduced populations of some species worldwide (Barnosky et al., 2011). More attention has been focused on imperiled vertebrates such as birds and mammals; however, many better-known invertebrates have also experienced significant declines (Taki and Kevan 2007). Global monitoring data of 452 invertebrate species have recorded a $45 \%$ decline in the past 40 years (Dirzo et al., 2014). Development, invasive species, and farming and grazing are the top three major threats to federally endangered and threatened insects in the United States (Wagner and Van Driesche, 2010).

Since the early 1900s, South Florida has experienced extensive development at the expense of natural ecosystems. Increased urbanization has reduced green space and threatened populations of species dependent on resources present in natural areas. Historically, subtropical rockland ecosystems covered a vast portion of Miami-Dade and Monroe Counties (Snyder et al., 1990). Subtropical dry forests (hardwood hammocks) are dense, evergreen forests dominated by broad-leaved trees (Snyder et al., 1990; Ross et al., 1992). Pine rocklands are fire-dependent, open habitats with pine trees in the overstory and a limestone rocky substrate (Snyder et al., 1990). Both ecosystems are found on higher ground, which has led to their demise (Alonso \& Heinen, 2011) as these higher 
areas are the most attractive for human habitation and use. Over $98 \%$ of the original pine rockland ecosystem outside of Everglades National Park has been destroyed by human activities. Development can modify the natural environment by loss of species (habitat simplification), increased matrix habitat (unsuitable habitat between remaining natural areas), and distance from viable habitats (loss of corridors). Consequently, many butterflies, other insects, and plants of south Florida's forests and pine rockland ecosystems have experienced significant population reductions with some species going extinct. Many imperiled species are now confined to state and federal parks and preserves, which are often underfunded and threatened by the effects of pollution, invasive species, and reduced natural buffer habitats. The protection of natural habitats and restoration of modified areas are two of the many solutions available to protect south Florida's and Earth's biodiversity (Hoekstra et al., 2005; Mathew and Anto, 2007; Oliver et al., 2010).

Rehabilitating greenspaces at schools, community centers, and neighborhoods in urban areas by planting native plants, removing invasive plants (including exotic lawn grass), and minimizing pesticide application can provide viable habitats that shelter common and rare butterflies from deleterious effects associated with urbanization (Ricketts, 2001; Brown Jr. and Freitas, 2002). With habitat rehabilitation, a depauperate school ground can become a biodiverse ecosystem. The imperiled atala butterfly (Eumaeus atala) was approaching extinction due to habitat loss and lack of accessible host plants (native cycads called "coontie"; Zamia integrifolia), but many coontie plantings in parks, at schools, and in neighborhoods helped atala butterflies rebound and recover in urban and suburban communities (Smith, 2002; Ramírez-Restrepo et al., 
2017). The atala butterfly is one of a few specialist butterfly species robust enough to inhabit human-dominated landscapes (Koi and Daniels, 2015; Ramírez-Restrepo et al. 2017); there are many other more sensitive butterfly species that are unlikely to establish populations beyond natural areas (Schultz and Dlugosch, 1999; Jameson, 2002; McElderry et al., 2015). Host plants are critical for all butterflies to maintain population viability (Vickery, 1995; Schultz and Dlugosch, 1999; Dennis et al., 2004), as adult females need to lay their eggs on plants suitable for the development of their larvae (caterpillars). Adult butterflies will be less abundant in areas if host plants are lacking (Koh and Sodhi, 2004; Mathew and Anto, 2007; Hammer, 2015), with the exception of migrating species. Imperiled butterfly species often depend on host plants specific in natural areas that are imperiled themselves (Schultz and Dlugosch, 1999; Salvato, 2003; McElderry et al., 2015). Outreach programs that implement native plant butterfly gardening by growing and establishing host plants in backyards, parks, and schoolyards may potentially help rare and endemic butterflies in south Florida (Mathew and Anto, 2007; Revathy et al., 2014; Ramírez-Restrepo et al. 2017).

\section{$\underline{\text { Insect Conservation, Science Enrichment, Constraints }}$}

Schools provide an opportunity to capitalize on integrative, place-based, botanical education by modifying their green spaces (Waliczek and Zajicek, 1999; Sobel, 2005). Teachers can use the school's surroundings as a framework in which students can both build their own learning and improve local environmental quality (Lieberman and Hoody, 1998; Sobel, 2005; Tatarchuk and Eick, 2011). Further activities by families and lifelong compassion and advocacy for the environment are also encouraged by creating 
schoolyard habitats (Feinsinger et al. 1997; Waliczek and Zajicek, 1999; Sobel, 2005).

Younger students tend to be more receptive to nature (Pyle, 2002; Fisher-Maltese, 2016), and elementary educators can align state standards with activities incorporating the butterfly garden (Culin, 2002; Tatarchuk and Eick, 2011), leading to greater environmental awareness later in students' lives (Culin, 2002; Sobel, 2005; Miller, 2005; Grunova et al., 2017).

Many studies have demonstrated the importance of school gardens as effective teaching tools inside and out of classrooms (Klemmer et al., 2005; Sobel, 2005; Libman, 2007). The hands-on, active learning approach connects students with the environment as they become cognizant of their surroundings (Waliczek and Zajicek, 1999; Pyle, 2002). For example, edible gardens can re-calibrate students' dispositions about food, particularly unpopular vegetables (Libman, 2007). Teachable moments and garden failures eventually transform to garden success if teachers scaffold their students to work through adversity and "fail forward" (Lieberman and Hoody, 1998; Waliczek and Zajicek, 1999; Culin, 2002; Settlage and Southerland, 2007). The capacity to raise butterflies at schools could make inconspicuous invertebrate species salient and facilitate potential action to protect them (Cutting and Tallamy, 2015; Ramírez-Restrepo et al. 2017).

South Florida is a particularly promising place for the use of butterfly gardens as part of a school curriculum. Unlike other regions of the US, the subtropical climate of south Florida allows people to observe butterfly activity year-round (Minno \& Emmel, 1993; Hammer, 2015). Developing native plant butterfly gardening at schools in Miami-Dade County creates an ecological schoolyard (Feinsinger et al. 1997), an opportunity to apply 
textbook material to the real-world, promote conservation (Caro et al. 2003), reduce plant blindness (Wandersee and Schussler 1999), and disrupt classroom monotony (Obboko and Hannington, 2014). Plant blindness, the inability to recognize plants in one's own environment, leads to the inability to appreciate and understand plants as important in ecosystems and for people (Balick and Cox, 1996; Wandersee and Schussler, 1999; Allen, 2003). Butterfly gardens can address both insect conservation and plant blindness in south Florida. In our study, we focus on native plants in butterfly gardens; because exotic plants thrive in south Florida, they are often planted in butterfly gardens because of their colorful, luxurious appeal, yet they may be less useful and even harmful to the environment (Reichard and White, 2001) or butterflies (Satterfield et al., 2015; Barriga et al., 2016). Native plant diversity, especially variety in host plants for butterflies, benefit butterfly heterogeneity and other insects dependent on native plants (Koh and Sodhi, 2004; Daniels, 2013).

Nature is not tidy and organized, but traditional societal ideology pressures people to manicure their lawns and gardens. A well-manicured garden is aesthetically pleasing to people, but might be functionally poor to the intended faunal guests (butterflies). What many people regard as weeds are shelter and food resources to a myriad of organisms including butterflies. But to persist, a butterfly garden has to have eye-appeal: disheveled butterfly gardens at schools may be deemed unsightly, and are at risk of elimination by unsympathetic administrators and staff responsible for maintaining the school grounds (Culin, 2002). 
$\underline{\text { South Florida native plant butterfly school garden study-with a focus on a charismatic }}$ $\underline{\text { rare species }}$

The "Schaus and Coastal Hardwood Hammock" curriculum unit encompassing the Schaus' swallowtail habitat enhancement project, local butterflies in South Florida, insect conservation, pine rockland and subtropical dry forest ecosystems, and native plant butterfly gardening was developed and refined for this study with the aid of veteran teachers (Clayborn et al., 2017). The curriculum unit followed educational best practices from the research literature and feedback from veteran teachers. We developed a 'gardening for butterflies' curriculum unit for children aged $10-11$ years old $\left(5^{\text {th }}\right.$ graders) using our understanding of the biology of one particular butterfly endemic to south Florida. The Schaus' swallowtail butterfly (Heraclides aristodemus ponceanus) was the first federally listed butterfly in 1976 and declared in danger of extinction in 1984 (Smith et al., 1994; Bibb and Hughes, 2007; FWS, 2017). Despite stable populations of Heraclides aristodemus subspecies in the Caribbean and Lucayan Archipelago, the Schaus' swallowtail butterfly, endemic to south Florida, is on the verge of extinction because of habitat loss and vulnerability to its isolation in Biscayne National Park and North Key Largo (Smith et al., 1994; Bibb and Hughes, 2007; FWS, 2017). The Schaus' swallowtail butterfly habitat enhancement project was initiated by scientists and staff at the National Park Service South Florida Caribbean Network (Whelan, 2011; Whelan and Atkinson, 2015). The habitat enhancement project restored degraded sections of subtropical dry forests on two islands in Biscayne National Park where Schaus swallowtail butterflies still occur (Clayborn et al. 2017). Conservation projects for rare 
and threatened species are more effective when combined with educational programs in local communities (Guiney and Oberhauser, 2009; Grunova et al., 2017).

The curriculum unit incorporated examinations into the project. Examinations not only pressure students to learn specific information on a given topic, they also help students retain more of the specific information, compared to that retained simply by restudying the information (Carpenter et al., 2008). A series of tests over time significantly increases retention of information (Carpenter et al., 2008). Post-tests only measure what students know at the end of a course/year/activity; without pre-testing, it is not possible to assess knowledge gained, or to compare experimental and control groups without bias (Dimitrov and Rumrill Jr, 2003; Lemieux and Allen, 2007). In addition, oneoff workshops, field trips to parks, museums, and zoos, and special guest speakers provide opportunities to engage and excite students about conservation biology (Brewer, 2002; Grajal et al., 2017). Nonetheless, continued immersion and participation (including mentorship and examinations) are crucial to enhancing knowledge retention, civic engagement, and environmental compassion (Kwan et al., 2017). Thus, we developed a 3-month long program to repeatedly engage students with butterflies and native plants.

The study's purpose was to measure the success of the curriculum unit in increasing student engagement, learning, awareness of, and advocacy for, butterflies and the environment. Students who participated in the curriculum unit were administered preand post-tests, pre- and post-surveys, pre- and post-interviews. We also looked at the influence of personal involvement (intervention) by giving students in the experimental group plants to take home, care for, and record observations, while control group students did not have that additional involvement. 
The objectives were to:

1) Incorporate a curriculum unit designed to address the plight of a rare butterfly, insect conservation, pine rockland and subtropical dry forest ecosystems, and native plant butterfly gardening in schools in Miami-Dade County;

2) Facilitate the construction of native plant butterfly gardens;

3) Increase the number of butterfly gardens in the extended communities; and

4) Assess both changes in attitudes/values and gains in content knowledge from the sequenced lessons in the curriculum unit and from extended plant and butterfly observations.

\section{METHODS}

$\underline{\text { Study area }}$

Miami-Dade County is the southernmost county on the mainland of the United States. The subtropical climate and proximity to the Lucayan Archipelago and Greater Antilles have contributed to south Florida's diverse flora and fauna. Many tropical Lepidoptera (butterflies, moths, and skippers) species reach their northern limits in south Florida, specifically Miami-Dade and Monroe Counties (Smith et al., 1994).

Miami-Dade County is also demographically diverse, with dominant Spanishspeaking populations from numerous countries in the Caribbean and South America. Three schools in Miami-Dade County participated in the study. Collectively, the schools follow a latitudinal gradient from North Hialeah south to Homestead, Florida (Figure 1). North Hialeah Elementary (NHE; 25 $51^{\prime} 40.42^{\prime \prime}$ N, 80 $16^{\prime} 15.37^{\prime \prime} \mathrm{W}$ ) is located in a densely packed urban, residential area with no natural areas and minimal green space 
near the school. Air Base K-8 Center (AB; 25 30'55.31” N, 80²3'57.01” W) and Whispering Pines Elementary (WPE; $25^{\circ} 35^{\prime} 27.58^{\prime \prime} \mathrm{N}, 80^{\circ} 20^{\prime} 05.88^{\prime \prime} \mathrm{W}$ ) are adjacent to pine rockland ecosystems. Both schools (AB and WPE) are also located within a region harboring protected environmentally endangered lands (EEL) and natural forest communities (Figure 1). Despite close proximity to these natural areas (EEL and nature forest communities), neither school (AB and WPE) actively engages their students in learning activities in these natural areas.

\section{$\underline{\text { Study preparation }}$}

Teachers, principals, and groundskeeper staff were given explicit information (project proposal, synopsis, and follow-up discussion) regarding the study at each school. Cooperation between teachers and the administration was essential to successfully execute the study. The study employed a quasi-experimental mixed methods approach (pre- and post-tests, pre- and post-surveys, pre- and post-interviews; Cook and Campbell, 1979; Tashakkori and Teddlie, 1998). Two $5^{\text {th }}$ grade classrooms ( 20 students per class) from each school were selected by convenience sampling and then each class was designated either the experimental treatment group or control treatment group. Both treatments followed the same protocol for the duration of the study; except, the experimental group at each school was given butterfly and flower identification plant guides, butterfly host and nectar plants, and a data sheet as part of the take-home portion of the study. All participants in the study completed consent forms approved by the Florida International University Institutional Review Board (FIU Institutional Review Board \# IRB-15-0080). Teachers used the signed consent forms to randomly select eight 
students from each classroom to be interviewed twice (pre- and post-interviews).

Teachers also completed the "Science Teaching Efficacy Belief Instrument" taken from Riggs and Enochs (1990).

\section{$\underline{\text { Initial assessments (Week One; September 2015) }}$}

During the first week of the study, the principal investigator (PI) introduced himself to the students at each school. Teachers explained the procedures for pre-survey interpretation and completion. Surveys (based on Likert's five-point scale; Likert, 1932) were coded for student confidentiality and also kept track of pre- and post-survey results. Statements spanned a range of topics about plants, animals, insects, and the environment (Table 1). Survey statements were reviewed and refined after consultation with several veteran teachers and $5^{\text {th }}$ grade students during the 2015 Spring semester. After everyone completed the survey, students were given time (25 minutes) to complete the pre-test. The pre- and post-test modeled standard exams given in class appropriate for $5^{\text {th }}$ graders. The content of the exam covered topics on south Florida ecosystems, plants, and butterflies. A variety of question types was employed including: 1) fill in the blank statements using a vocabulary box, 2) short answers, 3) multiple choice, 4) cause and effect, 5) sequencing, and 6) identification (see supplementary materials for copies of the pre- and post-test). After their class period ended, the eight students randomly selected by their teacher were interviewed individually by the PI. The interviews were held in a separate, quiet space away from other student distractions. Students were asked specific questions about school and leisure activities, garden space, and butterflies (Table 1). The method was repeated for each class during the first week of the study. 
$\underline{\text { Interactive classroom lecture and activities (Week Two; September 2015) }}$

The PI returned to each class and delivered an interactive presentation using PowerPoint ${ }^{\mathrm{TM}}$ and visual aids. The presentation was titled, "Imperiled butterflies of South Florida: Plight of the Schaus' swallowtail and other butterflies," and covered information on South Florida ecosystems, imperiled butterflies, insect conservation, and native plant butterfly gardening in Miami-Dade County (Clayborn et al., 2017). Students were engaged during the presentation with visual games and brief discussions, prompted by questions. Afterwards, students played a map game titled, "Place the Schaus' swallowtail butterfly in the right habitat" (see supplementary materials; Clayborn et al., 2017). Finally, students observed butterfly eggs, caterpillars, and native host and nectar plants. The PI informed students they would construct a butterfly garden at their school the following week.

Native plant butterfly garden construction (Week Three; September 2015)

Students from both groups (experimental and control), but as separate classes, were given space near the math and science classroom to construct their native plant butterfly garden. Information about each plant was introduced to each class, including: 1) species name, 2) preferred natural habitat, 3) morphology, and 4) ecological significance in the garden and ecosystem. The instructor and lead teacher facilitated the process as students worked in the garden (Figure 2). Students worked in groups and were assigned quadrants in the garden space. Garden tasks included: 1) weed removal, 2) soil preparation and supplementation, 3) planting of native plants (see Table 2 for list of plant species), 4) mulch addition, and 5) small, labeled flags (plant species name) used as markers adjacent 
to the plant. Students added rocks from the local area to demarcate their garden space and protect against lawn mowers and weed trimmers. Students watered and weeded the garden periodically, during the full length of the study.

Experimental group home project (Week Four; October 2015)

Students in the experimental group from each school participated in the home project. As a class assignment, students received a data sheet with instructions (see supplementary materials). Each student also received two identification guides, "Butterflies of Southeast Florida: A Guide to Common and Notable Species" (Minno, 2014; Figure 3) and "Wildflowers of Southeast Florida including The Florida Keys and Everglades National Park: A Guide to Common Native Species” (Hammer, 2012). Each student was given one native butterfly host plant (Passiflora suberosa) and one nectar plant (Salvia coccinea) to maintain at home. Plants were kept in one gallon pots with soil and mulch, and labeled with the common and scientific name.

Students were responsible for plant maintenance during the study. Plants were placed outside in a partial shade location and watered as needed by sticking a finger 3centimeters in the soil to gauge soil moisture. Once a week, students were required to monitor butterfly recruitment (identify butterfly species flying around or landing on the plants) and detect the presence of eggs, caterpillars, and chrysalises on the host plant. They were also encouraged to record the presence of animals on or near both plant species. Plant maintenance and observations lasted approximately 2 months. 


\section{$\underline{\text { Wrap-up (Final Week; December 2015) }}$}

The study concluded in the classroom. The procedure for the post-survey, post-test, and post-interview paralleled week one of the study. Students in the experimental group reported their observations that included animal activity on and near their host and nectar plants at home. The instructor collected the data sheets for further analyses; students kept their butterfly and plant identification guides. Students in the control group also received butterfly and plant identification guides for participating in the study (Hammer, 2012; Minno, 2014).

\section{DATA ANALYSIS}

A Shapiro-Wilk test for normality was performed on the pre- and post-test scores. Pre- and post-tests were analyzed with paired sample $t$-Tests. A one-way Analysis of Covariance (ANCOVA) was performed to assess the influence of the intervention (students in the experimental group maintaining host and nectar plants) on post-test scores with pre-test scores as the covariate.

An exploratory factor analysis (EFA) was performed to identify strongly correlated survey statements, which were loaded into factors. Factors with one statement were eliminated, two or more statements per factor were kept. Students' response scores from strongly correlated survey statements were averaged for each factor. A Quade's rank analysis of covariance was utilized to assess the influence of gardens present at the place of residence before the study commenced, intervention of taking host and nectar plants home, and type of students' residence for each factor with pre-survey scores as the covariate (Quade, 1967). All data analyses were conducted using IBM SPSS Statistics 24 
(Statistical Package for the Social Sciences). Garden data reported in the pre- and postsurveys were summarized and reported in a table (Table 8).

Interview responses were recorded using the GarageBand program (Apple Inc.) and later transcribed by hand. An inductive thematic analysis approach was applied to identify themes that emerged from the data (Boyatzis, 1998; Braun and Clarke, 2006; Padgett, 2008). Theme identification and analysis followed a six-step process: 1) detailed notes were taken on all responses, 2) initial codes were generated from relevant pieces of information, 3) candidate themes were developed for further analysis, 4) candidate themes, initial codes, and detailed notes were reviewed by multiple people for coherence, 5) themes were defined, named, quantified, and analyzed, and 6) findings were described and reported (Boyatzis, 1998; Braun and Clarke, 2006).

\section{RESULTS}

$\underline{\text { Science teaching efficacy self-assessment instrument }}$

Each participating teacher (one at each school) was generally positive about their ability to teach science in the classroom. More importantly, they were confident in answering questions and piquing student interests in science. The teachers at Air Base and Whispering Pines reported teaching other subjects better than they taught science. Teacher responses varied regarding external factors such as students' ability to learn and motivation in science. Internal factors, what teachers could control when teaching science, were generally more consistent and positive (Table 3). 


\section{$\underline{\text { Knowledge acquisition }}$}

Across all schools, students in the experimental group scored significantly higher on the post-test (pre-test mean $=0.68, \mathrm{SD}=0.16$; post-test mean $=0.80, \mathrm{SD}=0.13 ; t=-$ $6.301, \mathrm{p}<0.001, \mathrm{n}=60$ ), compared to no significant increase in the control group (pretest mean $=0.63, \mathrm{SD}=0.15 ;$ post-test mean $=0.65, \mathrm{SD}=0.17 ; t=-1.239, \mathrm{p}=0.220, \mathrm{n}=$ 60; Figure 4). Each experimental group at each school significantly scored higher on the post-test compared to no significant increase for control groups (Air Base: experimental group, pre-test mean $=0.75, \mathrm{SD}=0.09$; post-test mean $=0.86, \mathrm{SD}=0.08$; control group, pre-test mean $=0.74, \mathrm{SD}=0.09 ;$ post-test mean $=0.77, \mathrm{SD}=0.10 ;$ paired sample $t$-tests, experimental group: $t=-3.871, \mathrm{p}<0.001, \mathrm{n}=21$; control group: $t=-0.937, \mathrm{p}=0.360, \mathrm{n}$ = 20; Figure 5. North Hialeah Elementary: experimental group, pre-test mean $=0.73$, $\mathrm{SD}=0.10 ;$ post-test mean $=0.81, \mathrm{SD}=0.15$; control group, pre-test mean $=0.55, \mathrm{SD}=$ 0.15 ; post-test mean $=0.57, \mathrm{SD}=0.17$; paired sample $t$-tests, experimental group: $t=-$ 2.403, $\mathrm{p}=0.028, \mathrm{n}=18$; control group: $t=-0.573, \mathrm{p}=0.573, \mathrm{n}=20$; Figure 6 .

Whispering Pines Elementary: experimental group, pre-test mean $=0.55, \mathrm{SD}=0.19$; post-test mean $=0.72, \mathrm{SD}=0.12$; control group, pre-test mean $=0.61, \mathrm{SD}=0.14$; posttest mean $=0.63, \mathrm{SD}=0.17$; paired sample $t$-tests, experimental group: $t=-4.705, \mathrm{p}<$ 0.001, $\mathrm{n}=21$; control group: $t=-0.701, \mathrm{p}<0.492 ; \mathrm{n}=20$; Figure 7).

The intervention applied to the experimental groups, individual students nurturing and observing their own personal host and nectar plants at home, had a significant effect on post-test score gains. Overall, experimental groups' post-test scores were significantly higher compared to the control groups for all schools combined (control group mean $=$ $0.708, \mathrm{SD}=4.427 ;$ experimental group mean $=3.721, \mathrm{SD}=4.574 ; d f=1, F=25.771, \mathrm{p}$ 
$<0.001$; Table 4; Figure 4). Post-test scores were also significantly higher for experimental groups at individual schools. Air Base: control group mean $=0.850, \mathrm{SD}=$ 4.056; experimental group mean $=3.190, \mathrm{SD}=3.777 ; d f=1 ; F=9.926, \mathrm{p}=0.003 ;$ Table 4; Figure 5. North Hialeah Elementary: control group mean $=0.725, \mathrm{SD}=5.660$; experimental group mean $=2.458, \mathrm{SD}=4.341 ; d f=1, F=6.203, \mathrm{p}=0.018 ;$ Table 4; Figure 6. Whispering Pines Elementary: control group mean $=0.550, \mathrm{SD}=3.509$; experimental group mean $=5.333, \mathrm{SD}=5.195 ; d f=1, F=10.986, \mathrm{p}=0.002 ;$ Table 4; Figure 7.

\section{Attitudes before and after-survey results}

After performing the EFA, the Kaiser-Meyer-Olkin (KMO) measure of sampling adequacy was 0.688 including a significant Bartlett's test for Sphericity $(\chi=946.401, \mathrm{df}$ $=378, \mathrm{p}<0.001)$. The KMO Test measures how suited the data are for Factor Analysis. The KMO value of 0.688 is sufficient to justify additional analyses. The Bartlett's test for Sphericity checks for redundancies between variables that can be summarized with some factors and must be less than $\mathrm{p}<0.05$ to perform the Factor Analysis. Fourteen of the 28 statements loaded into 6 Factors; however, one Factor included only one statement and was removed from analysis (Table 5).

Quade's test revealed no significant changes in attitudes for any of the Factors based on intervention, type of residence, or presence of gardens at home for all schools combined (Table 5). However, the intervention was significant regarding post-survey score changes between experimental and control groups at Whispering Pines Elementary for Factor 4 (Interconnectedness (Bees and Plants): $F=8.213, \mathrm{p}=0.007$ ) and Factor 5 
(Anthropogenic Harm: $F=6.155, \mathrm{p}=0.018$; Table 5). Post-survey scores for Factor 4 Interconnectedness (Bees and Plants) reported an increase for the control group (presurvey mean $=3.816, \mathrm{SD}=0.831 ;$ post-survey mean $=4.237, \mathrm{SD}=0.547$; Table 6 ) and decrease for the experimental group (pre-survey mean $=4.476, \mathrm{SD}=0.732$; post-survey mean $=4.238, \mathrm{SD}=0.453$; Table 6). Post-survey scores for Factor 5 - Anthropogenic Harm reported a decrease for the control group (pre-survey mean $=4.404, \mathrm{SD}=0.453$; post-survey mean $=4.123, \mathrm{SD}=0.594$; Table 6 ) and an increase for the experimental group $($ pre-survey mean $=3.635, \mathrm{SD}=0.981 ;$ post-survey mean $=4.175, \mathrm{SD}=0.985$; Table 6). Air Base and North Hialeah Elementary showed no significant changes in attitude for any of the Factors based on intervention, type of residence, or presence of gardens at home (Table 5).

\section{$\underline{\text { Attitudes before and after-interview results }}$}

Eight randomly selected students were interviewed from each class twice (pre- and post-interviews) during the study ( $\mathrm{n}=48$ students, $\mathrm{n}=96$ interviews). As a result of technical difficulties with the program GarageBand, some of the recorded interviews were corrupted. In the experimental group only, six post-interviews from North Hialeah Elementary and two pre- and post-interviews from Whispering Pines Elementary were corrupted; however, the remaining recorded interviews were used for analysis and interpretation ( $\mathrm{n}=48$ students, $\mathrm{n}=86$ interviews). Themes were derived and quantified from students' responses. If $20 \%$ or more of the students from each group (control or experimental) gave a response that fit into a particular theme, then that theme was reported in the results section. 


\section{1) What kind of activities do you enjoy doing outdoors?}

Three themes derived from this question included: 1) Sports, 2) Random Play, and 3)

Gardening/Yard Exploration. More students in the control group (pre - 67\%, post - 63\%) engaged in "Sports" than the experimental group (pre $-41 \%$, post $-50 \%$ ). The percentage of students, in the experimental group, engaged in "Random Play" outside increased $19 \%$ during post-interviews (pre $-50 \%$, post $-69 \%$ ), but remained the same in the control group (pre $-58 \%$, post $-58 \%$ ). Responses regarding “Gardening or Yard Exploration" in the control group increased (pre $-25 \%$, post $-38 \%$ ), but responses decreased (pre $-50 \%$, post $-31 \%$ ) for the experimental group during post-interviews (Table 7).

\section{2) What kind of activities do you like to do indoors?}

In the control group, five themes derived which included: 1) Read/Study, 2)

Drawing/Arts \& Crafts, 3) Playing, 4) Electronics/Computer, and 5) TV. More than 40\% of the students reported "Playing" during both interviews (pre $-46 \%$, post $-42 \%$ ). Twelve percent more students reported "Reading or Studying" during post-interviews (pre $-13 \%$, post $-25 \%$ ). More students use the "Computer or Electronics" (pre $-42 \%$, post $-29 \%$ ) than watch "TV" (pre $-21 \%$, post $-21 \%$ ). "Drawing or Arts \& Crafts" declined by $12 \%$ (pre $-29 \%$, post $-17 \%$; Table 7 ).

In the experimental group, the theme Read/Study was omitted because less than $20 \%$ responded during both interviews. "Playing" was the most popular theme (pre $-50 \%$, post $-56 \%$ ). Forty-five percent of students reported watching "TV" during preinterviews, but watching "TV" declined 20\% during post-interviews (25\%). The use of 
the "Computer or Electronics" increased during post-interviews (pre -27\%, post -38\%).

"Drawing or Arts \& Crafts" declined by 4\% (pre - 23\%, post - 19\%; Table 7).

\section{3) How much time do you spend outside during school?}

Median and mode values were selected because the data were skewed to the right. Students in the control group reported a range from 15 - 120 minutes, median and mode values at 45, 45 minutes during pre-interviews. Post-interview responses included a range from $15-180$ minutes, median and mode values of 45,30 minutes. Students in the experimental group reported a range from $10-180$ minutes, median and mode values at 60, 60 minutes during pre-interviews. Post-interview responses included a range from 15 - 120 minutes, median and mode values at 52.5, 60 minutes (Table 7). Students reported being outside for Physical Education (PE) and transitioning to and from the cafeteria.

\section{4) How much time do you spend outside after school?}

Once again, median and mode values were selected because the data were rightskewed. Students in the control group reported a range from 5-300 minutes, median and mode values at 105, 120 minutes during pre-interviews. Post-interview responses included a range from $10-300$ minutes, median and mode values of 60, 90 minutes. Students in the experimental group reported a range from $30-240$ minutes, median and mode values at 60, 60 minutes during pre-interviews. Post-interview responses included a range from $10-240$ minutes, median and mode values at 60,120 minutes (Table 7). 


\section{5) Can you describe a butterfly garden?}

Four themes derived from question \#5 included: 1) Plants, 2) Flowers, 3) Host Plants, and 4) Has Butterflies. More than 50\% of the respondents in the control group mentioned "Plants" (58\%) and "Has Butterflies" (54\%) during pre-interviews. Thirty-eight percent mentioned "Flowers". During post-interviews, "Plants" (67\%) and "Flowers" (58\%) were the most popular responses. The response "Has Butterflies" declined 12\% (pre-54\%, post $-42 \%)$. Twenty-one percent mentioned "Host Plants" during post-interviews, an increase of $13 \%$ (pre $-8 \%$, post $-21 \%$ ).

In the experimental group, "Flowers" (41\%) was the most popular response during pre-interviews. More than $30 \%$ of the respondents mentioned "Has Butterflies" (36\%) and "Plants" (32\%). Twenty-three percent mentioned "Host Plants". During postinterviews, there were modest increases for "Plants" (pre $-32 \%$, post $-50 \%$ ) and "Flowers" (pre - 41\%, post - 50\%), slight increase for "Host Plants" (pre - 23\%, post $25 \%$ ), and modest decrease for "Has Butterflies" (pre $-36 \%$, post $-25 \%$ ). In both groups, the only specific butterfly and host plant named by several students were the iconic monarch butterflies and milkweed plants (Table 7).

\section{6) Name some things you need to attract butterflies to a garden.}

In the control group, three themes were derived which included: 1) Flowers, 2) Plants, and 3) Host Plants. "Flowers" and "Plants" were considered basic responses because it demonstrated a rudimentary understanding of components used to attract butterflies. The theme "Host plants" was considered an advanced response because it demonstrated a more sophisticated understanding of plants used to attract butterflies. More than 50\% of 
the respondents mentioned "Flowers" during both interviews (pre $-58 \%$, post $-54 \%$ ). Thirty-eight percent of the respondents mentioned "Plants" during both interviews. Twenty-five percent more respondents mentioned "Host Plants" during post-interviews (pre $-13 \%$, post $-38 \%$; Table 7).

In the experimental group, five themes were derived which included: 1) Flowers, 2) Plants, 3) Host Plants, 4) Nectar Plants, and 5) Milkweed. The most popular responses during pre-interviews were "Flowers" (50\%) and "Nectar Plants" (45\%). The percentage of students that mentioned "Flowers" declined substantially during post-interviews (pre $50 \%$, post $-13 \%$ ). There was a modest decrease for "Nectar Plants" (pre $-45 \%$, post -

38\%). Thirty-two percent of respondents mentioned "Host Plants" during pre-interviews which increased to $44 \%$ during post-interviews. The percentage of respondents that mentioned "Plants" (pre $-10 \%$, post - 38\%) and "Milkweed" (pre - 10\%, post $-25 \%$ ) also increased substantially during post-interviews. As mentioned in the previous paragraph, the themes "Host plants" and "Nectar Plants" were considered advanced responses because they demonstrated a more sophisticated understanding of plants used to attract butterflies. Both groups mentioned "Milkweed" (a higher percentage of respondents mentioned "Milkweed" in the experimental group) as attractors for butterflies, but no other specific plant name (Table 7).

\section{7) Do you have a garden outside of school?}

A higher percentage of students in the control group already had gardens at home than did students in the experimental group. During post-interviews, students reported a modest decrease in the number of gardens at home for both groups; however, the control 
group still had a higher proportion of gardens (control group: pre $-83 \%$, post $-71 \%$;

experimental group: pre $-50 \%$, post $-44 \%$ ). Some students reported their garden was located at their abuela's (grandmother) house who they visited frequently (Table 7).

\section{8) Tell me about some of the plants in your garden.}

Two group of plants derived from statement \#8 included: 1) Edible Plants and 2) Ornamental Plants. Many students reported gardens of mixed use (edible and ornamental plants). In the control group, 50\% of the respondents with gardens at home or close relative's home mentioned "Edible Plants" during pre-interviews; however, that declined by $25 \%$ during post-interviews. "Ornamental Plants" were mentioned by $38 \%$ of the respondents during pre-interviews and increased to 50\% during post-interviews.

In the experimental group, $41 \%$ of respondents mentioned "Ornamental Plants" during pre-interviews which declined to $31 \%$ during post-interviews. There was a moderate increase for "Edible Plants" during post-interviews (pre-18\%, post - 25\%). Students reported growing tomatoes, mangos, basil, other herbs, and peppers. Flower plants included orchids, roses, lilies, tulips, and sunflowers. Few respondents mentioned butterfly plants in their garden; however, if they did it was milkweed (Table 7).

\section{9) Would it be easy or difficult for animals and plants to survive in your neighborhood, briefly explain?}

In the control group, more than half of the respondents reported, "It would be easy for animals and plants to survive in their neighborhood" during both interviews (pre $-54 \%$, post $-54 \%)$. Twenty-nine percent stated, "It would be difficult or somewhat difficult 
for animals and plants to survive in their neighborhood" during pre-interviews, but the proportion of responses increased during post-interviews (42\%). Two themes were derived to explain the ease or difficultness of animal and plant survivorship in their neighborhoods which were 1) Lots of Green Space and 2) Lack of Green space. Twentynine percent of respondents mentioned "Lots of Green Space" during pre-interviews which increased to 38\% during post-interviews. "Lack of Green Space" also increased during post-interviews (pre $-8 \%$, post $-21 \%$; Table 7 )).

In the experimental group, 59\% of respondents reported, "It would be easy for animals and plants to survive in their neighborhood" during pre-interviews which increased to $69 \%$ during post-interviews. Thirty-six percent stated, "It would be difficult or somewhat difficult for animals and plants to survive in their neighborhood" during pre-interviews, but the proportion of responses decreased during post-interviews (31\%). Two themes were derived to explain only the ease of animal and plant survivorship in their neighborhoods which were 1) Lots of Green Space and 2) Friendly Neighbors. Forty-one percent of respondents mentioned "Lots of Green Space" during pre-interviews which increased to 50\% during post-interviews. "Friendly Neighbors" also increased during post-interviews (pre $-18 \%$, post $-25 \%$; Table 7).

Another notable theme, though below the $20 \%$ of respondents' threshold, was "Unruly Domesticated Animals (Dogs/Cats)". Several students, in the experimental group, mentioned pets destroyed their nectar and host plants during the study. 
10) Describe some reasons butterflies and other insects are disappearing, going extinct.

In the control group, five themes derived included: 1) No Food, 2) Predators, 3)

Pesticides, 4) People Harming Them, and 5) Habitat Loss. These five themes explained the disappearance or extinction of butterflies and other insects perceived by students (during the current study) as reasons butterflies and other insects were disappearing.

During pre-interviews, "No Food" was the most popular response (38\%), but declined during post-interviews (21\%). Students' responses for "People Harming Them" (pre $33 \%$, post $-42 \%$ ) and "Habitat Loss" (pre $-33 \%$, post $-42 \%$ ) mirrored each other.

Responses for "Predators" remained the same (pre $-21 \%$, post $-21 \%$ ), but "Pesticides" markedly increased during post-interviews (pre $-8 \%$, post $-25 \%$; Table 7 ).

In the experimental group, four themes derived included: 1) No Food, 2) Pesticides, 3) People Harming Them, and 4) Habitat Loss. "People Harming Them" was the most popular theme during pre-interviews (50\%), but decreased by $25 \%$ during postinterviews. Thirty-six percent of respondents reported "Habitat Loss" during preinterviews which increased to 56\% during post-interviews. Thirty-two percent of respondents reported "No Food" during pre-interviews which decreased to 25\% during post-interviews. There was a $15 \%$ increase in the percentage of students reporting "Pesticides" during post-interviews (pre $-10 \%$, post $-25 \%$; Table 7).

\section{1) Would you recommend a butterfly garden to a friend, can you explain?}

The majority of respondents from both groups said they would recommend a butterfly to a friend. Several students in the control and experimental groups said "No" during pre- 
interviews (control: pre - 8\%; experimental: pre - 14\%); however, all students said "Yes" during post-interviews. Themes derived from the responses included: 1) Increase Butterfly Population, 2) Like Butterflies, and 3) Help Endangered Butterflies. In the control group, the most popular theme was "Like Butterflies" (pre $-50 \%$, post $-42 \%$ ). Twenty-five percent of respondents reported "Increase Butterfly Population" during preinterviews which declined to 17\% during post-interviews. The theme "Help Endangered Butterflies" greatly increased during post-interviews (pre $-8 \%$, post $-21 \%$; Table 7 ).

In the experimental group, the most popular theme was "Like Butterflies" during preinterviews, but drastically decreased during post-interviews (pre $-50 \%$, post $-19 \%$ ). Twenty-seven percent of respondents reported "Increase Butterfly Population" during pre-interviews which increased during post-interviews (38\%). The percentage of respondents that contributed to the theme "Help Endangered Butterflies" more than doubled during post-interviews (pre $-14 \%$, post $-31 \%$; Table 7 ).

\section{Butterfly gardens in the extended community}

There was a net gain of eight new home gardens reported by students in the experimental group compared to a net loss of two in the control group (Table 8). Almost every student in the control group at North Hialeah Elementary had a garden before this study commenced; however, they reported two less gardens at the end of the study. The other control groups maintained the same number of gardens. Each school in the experimental group increased the number of gardens at home by two $(\mathrm{AB})$ or three (NHE, WPE). 
Despite a net increase of eight gardens in the experimental group, both groups had a net increase of five butterfly gardens after the study. North Hialeah Elementary students in both groups reported a dramatic increase in the number of butterfly gardens at home $($ Control Group = 5, Experimental Group = 4; Table 8).

\section{DISCUSSION}

A compassionate, efficacious, and knowledgeable teacher can influence and enhance students' interests in various topics and activities especially at a young age (Supovitz and Turner, 2000; Kazempour, 2014; Knaggs and Sondergeld, 2015). Studies have demonstrated student academic performance is strongly correlated to effective teachers (Stronge, 2013; Muñoz et al., 2013). All participating teachers were confident in their abilities to teach and answer questions relating to science (Table 3). However, responses varied regarding external factors such as students' academic achievement and motivation (statement \#1, \#14,\#25, Table 3). Each participating teacher felt self-efficacious in science, but responses differed when self-efficacy in science was applied to students (Table 3). A self-efficacious teacher in science is more likely to engage students in activities that reach beyond the textbook (Muñoz et al., 2013; Kazempour, 2014; Knaggs and Sondergeld, 2015) and be open to activities such as butterfly gardening and exploration (Culin, 2002, Fisher-Maltese, 2016).

Overall, students in the experimental groups performed better on the post-test than students in control groups over the course of this teaching unit. Looking at the schools individually, the knowledge gain derived from the intervention was significantly higher for the experimental groups. The host and nectar plant intervention was a challenging 
component to the study. Parental involvement was necessary to assist students and fulfill essential needs such as watering, important in Miami's subtropical climate. Additionally, plant survivorship and growth varied among the student participants. Most students nurtured and maintained their nectar and host plants, but some plants died because of unruly domesticated pets, lack of water, and neglect. Several interviewed students did report unruly animals, particularly dogs and cats, could harm and disturb butterflies and other insects in the neighborhood. Another constraint was butterfly recruitment; students were excited to potentially attract butterflies, but some were more successful than others. Local environmental factors may have contributed to the success of some students, such as proximity to natural areas, other established gardens, and other plants in the yard or garden (Koh and Sodhi, 2004; Mathew and Anto, 2007; Hammer, 2015; Olivier et al., 2016). While $100 \%$ success in butterfly recruitment for all students in the experimental group was unlikely, it is a continued target goal for future studies. High butterfly activity at home, and more class time integrating the school butterfly garden into student activities, could also have influenced students' attitudes about nature and insects.

Descriptively, post-survey results revealed most students (in both experimental and control groups) liked and valued animals, plants, and the environment, and were less interested in insects (Table 6; Figure 8). Independent variables (groupings) such as the presence of established gardens at home before the study, host and nectar plants intervention, and type of residence (house and apartment) did not significantly influence post-survey results when all schools were grouped together (Table 5). Individually, host and nectar plants intervention did significantly influence post-survey results for Factor 4 - Interconnectedness (Bees and Plants) and Factor 5 - Anthropogenic Harm at 
Whispering Pines Elementary (Table 5). Regarding Factor 4 - Interconnectedness (Bees and Plants), there was a modest increase ( $3.816 \pm 0.831$ to $4.237 \pm 0.547$ using the Likert Scale) for the control group and slight decrease $(4.476 \pm 0.732$ to $4.238 \pm 0.453)$ for the experimental group (Likert, 1932; Table 6). The control group expressed significant positive growth in their disposition towards bees and people and plants being important to the environment. The experimental group experienced a significant positive increase in disposition (3.635 \pm 0.981 to $4.175 \pm 0.985$ ) for Factor 5 - Anthropogenic Harm compared to a modest decrease for the control group $(4.404 \pm 0.453$ to $4.123 \pm 0.594$; Table 6). Overall, students valued environmental conservation, but were less interested in insects (Table 6; Figure 8). Survey results suggested young students already had a strong appreciation for the environment (Pyle, 2002), which can be harnessed by teachers and educators (Brewer, 2002; Culin, 2002; Tatarchuk and Eick, 2011). Efforts in demystifying insects as gross, annoying pests, but an essential component to the biological processes on Earth should be taught inside and out the classroom (Matthews et al., 1997; Rader et al., 2016).

Students' interviews provided an opportunity to share perceptions about themselves, hobbies, butterflies, gardens, and the environment. Type of activities and time spent outside often relates to connection to the environment. For example, students engaged in nature (exploring, gardening, playing) are more likely to advocate for its protection (Wells and Lekies, 2006; Cheng and Monroe, 2012; Broom, 2017). The most popular outdoor activities were sports, random play, and gardening and yard exploration (Table 7). Random play included activities such as tag, hide and seek, monkey bars, playing with their pet, and erratic running. Some students mentioned they enjoyed gardening with their 
parent(s) or abuela (grandmother); while other students observed plants and animals in the yard. Only a few students expressed interest in insects specifically butterflies for outdoor play. Overall, sports and random play were mentioned more than gardening or yard exploration, yet a third or more students still engaged in gardening or yard exploration (Table 7). Urban communities can constrain activities involving the natural environment (Miller, 2005). Time spent outside is another indicator whether students are more likely to engage in and appreciate the natural environment (Miller, 2005; Wells and Lekies, 2006; Broom, 2017). On the basis of wide-ranging responses regarding time spent outdoors, disjointed responses indicated students' sense of time is not well developed yet; however, using their responses (median values), students were outside from 60 - 105 minutes (Table 7).

Popular indoor activities were reading/studying, drawing/arts \& crafts, playing, electronics/computer, and TV (Table 7). Students, in both groups, reported indoor playing the most compared to other activities. They engaged in electronics or a computer and watched TV; however, we do not know the amount of time dedicated to each activity which would have been valuable information. The proportion of students engaged in reading or studying was low except for the control group during post-interviews when it increased to $25 \%$ (pre $-13 \%$, post $-25 \%$; Table 7). Overall, students enjoyed playing indoors and out. The act of "playing" could be harnessed as a "means to an end" to encourage exploration and inquiry in the butterfly garden, school grounds, and home backyard to promote environmental stewardship (Basile and White, 2000; Sobel, 2005; Jacobi-Vessels, 2013; Fisher-Maltese, 2016). 
At the schools, students' responses varied describing time spent outside. Once again, interpreting time appeared disjointed because students at the same school in the same class would give wide-ranging responses; however, there was a consensus regarding PE. They reported going outdoors mainly to engage in PE for 45 - 60 minutes (median values; Table 7). Physical education is important at school for fitness, cooperation, relief from the classroom, character development, and discipline (Bailey, 2006; Mahar et al., 2006); however, students are less likely to connect with the wild environment during PE.

Students were familiar with basic components of a butterfly garden. They mentioned butterfly gardens harbored plants, flowers, host plants, and butterflies (Table 7). Specific butterfly attractors mentioned were flowers, plants, and host plants. A higher proportion of students in the experimental group mentioned host plants as a butterfly attractor than students in the control group during pre- and post-interviews (Table 7). Host plants are essential for a successful, functional butterfly garden (Dennis et al., 2004; Daniels, 2013; Hammer, 2015). Student recognition of host plants detailed a better understanding of enticing butterflies to visit a garden as well as of the life history of butterflies. Some students mentioned milkweed, which is a popular host plant for monarchs and commonly planted at schools and home gardens. More than half of the students in the control group mentioned flowers (Table 7). Some flowers are great attractors for butterflies, but the term "flower" is a generic, loose term. The term "nectar plant" is more specific and focuses on plants that produce food resources for various pollinators including butterflies (Hammer, 2015). The combination of nectar and host plants are superior attractors for butterflies. Post-interview responses from the experimental group demonstrated a deeper understanding for attracting butterflies compared to the control group (Table 7). No 
interviewed students in both groups mentioned specific names of butterfly plants other than milkweed.

More students in the control group had gardens outside of school than students in the experimental group. Their gardens consisted mainly of edible and ornamental plants. Few gardens contained butterfly plants; however, if a butterfly plant was mentioned, it was milkweed. Tropical milkweed (Asclepias curassavica), an exotic plant species, is a seasonal plant commonly sold at plant nurseries in South Florida. To find other host plants requires research. Local native plant nurseries often have large selections of butterfly host and nectar plants, but many consumers might not be aware of them. Informed teachers can encourage students to learn more about host and nectar plant diversity to enrich school and home gardens.

In both groups, more than half of the students stated animals and plants would have an easy time surviving in their neighborhood because of abundant green space (Table 7). During the interviews, students were able to make the connection between abundant green space (the presence of herbaceous and woody plants) and wildlife presence, which was represented in the post-survey results for Factors 4 - Interconnectedness (Bees and Plants) and Factor 5 - Anthropogenic Harm (Table 6, Figure 8). Consequently, students were less specific regarding quality of green space. "We have a lot of plants", "a lot of flowers", or "we have trees in the neighborhood", were common responses associated with abundant green space. Some students in the experimental group also mentioned being surrounded by friendly neighbors which depicted a positive atmosphere of community and communication (Table 7). Both are necessary to promote and spread pragmatic environmental stewardship throughout the community (Sobel, 2005). During 
post-interviews, a higher proportion of students in the control group recognized that lack of green space was a reason animals and plants would have a difficult time surviving in their neighborhood (Table 7). Overall, many students had a somewhat difficult time stating concrete reasons plants and animals could or could not survive in their neighborhood. Nature within urban ecosystems is a relatively new concept as more people are becoming cognizant of synurbization (adaptation of wildlife to urban environments; Andrzejewski et al., 1978; McIntyre et al., 2008; Ramírez-Restrepo et al., 2017).

During post-interview results, a higher proportion of students, in both groups, recognized habitat loss and pesticides were deleterious to butterflies and other insects (Table 7). Lack of food was a popular response from students, in both groups, during preinterviews; however, the "lack of food" responses decreased during post-interviews (Table 7). One conjectural reason for the "Lack of Food" response might relate to monarch butterflies and milkweed. On the basis of responses to interview questions \#5, \#6 and statement \#8, some students were familiar with monarch butterflies and their host plant milkweed. We cannot deduce if students learned about monarchs and milkweed at home, school, plant nursery, or educational center before the study; however, the monarch story does detail population declines as a consequence of declining milkweed (lack of food for the caterpillars) along their migratory route (Howard and Davis, 2009; Guiney and Oberhauser, 2009; MonarchWatch.org. 2017). Students were also aware people could negatively harm butterflies and other insects through insect collections for money (butterfly wings) and directly killing them if perceived a nuisance (Table 7). 
During the pre-interviews, several students in both groups stated they would not recommend a butterfly garden to a friend because their friends would have no interest in it. Three months later all interviewed students stated they would recommend a butterfly garden to a friend (Table 7). Students in the experimental group thought more butterfly gardens could increase butterfly populations and help endangered butterflies. Students in the control group liked butterflies and wanted to share the beauty of butterflies and butterfly gardens with friends and family. They also wanted to increase butterfly populations and help endangered butterflies. Interviewed students were conscience of the negative effects associated with urban environments and people towards local flora and fauna. People can directly harm insects by squashing them or using pesticides or indirectly through development and removal of viable greenspace. The pre- and postsurvey results demonstrated students were less interested in insects and insects in their space (Figure 8); however, post-interview responses indicated students were willing to advocate for butterflies through the use of butterfly gardens (Table 7). School butterfly gardens can excite students to care more about their local flora and fauna, yet greening of communities is more sustainable in maintaining and attracting various wildlife including butterflies (Savard et al., 2000; Rudd et al., 2002; Koh and Sodhi, 2004; Nowak and Dwyer, 2007).

There was a net increase of eight gardens in the experimental group and a net loss of two gardens in the control group; however, each group had a net increase of five butterfly gardens (Table 8). At North Hialeah Elementary, both groups dramatically increased their number of butterfly gardens at home compared to the other schools, but data in Table 8 reveal some confusion in the definition of what constitutes a butterfly garden, and 
whether or not butterfly gardens were combined with edible or flower gardens. In the future, students should provide evidence of their gardens at home with pictures which can be assessed, clearly defined, and compared.

Giving students plants to take home presented an opportunity to motivate students to expand gardens beyond the school grounds. Native host and nectar plants were grown by the first author from seed and nurtured in a greenhouse until ready for use. Student ownership provided additional care and experiential learning to monitor plant growth and insect activity over time. However, individual plant observations and maintenance at home were likely more successful with parent, teacher, and peer support, which should be integrated in lesson plans or curriculum units (Culin, 2002; Tatarchuk and Eick, 2011). Teacher scaffolding can push the experiential process and teach students how to think based on acquired knowledge from the science content and their own observations in butterfly gardens at school and home (Brewer, 2002; Settlage and Southerland, 2007; Tatarchuk and Eick, 2011).

\section{CONCLUSION}

Teachers can use butterfly gardens to expand knowledge gained from school literature using various teaching techniques such as discovery, inquiry-based, and conceptual change strategies (Lieberman and Hoody, 1998; Skelly and Bradley, 2000; Tatarchuk and Eick, 2011; Settlage and Southerland, 2007). Teachers can integrate a myriad of scientific and mathematical components presented in the butterfly garden to their lesson plans that follow state education guidelines; in Florida, these are the Next Generation Sunshine State Standards (NGSSS). Ideally, service learning and 
experimental projects in the garden will prepare students for the Grade 5 Science and Math standardized exams (in Florida, the FCAT).

Many teachers may need support to effectively integrate butterfly garden activities into valuable class time (Skelly and Bradley, 2000; Brewer, 2002; Culin, 2002). Collaboration with experts from local universities, other schools, nature organizations, and government organizations brings not only some expertise to the school, but can make students aware of potential future careers in science and education (Solter, 1997; Brewer, 2002; Miller, 2005). Ongoing exposure to the school gardens through maintenance and class assignments can help students achieve academically, become socially aware about environmental quality, and create social capital where students work for the collective good of their community (Waliczek and Zajicek, 1999; Sobel, 2005).

Butterfly gardening goes beyond butterflies, as many other species of animals are also attracted to the garden (Minno and Emmel, 1993; Hammer, 2015). Students might develop an appreciation for preservation at their school butterfly garden becoming more protective of the very plants they nurtured into the ground.

South Florida and the Keys are ideal locations for human habitation at the expense of native wildlife. Through education for the willing, butterflies can be used as flagship species (Pe'er and Settele, 2008; Guiney and Oberhauser, 2009), as most people like butterflies, so they provide attractive models for conservation (Ramírez-Restrepo et al., 2017; Clayborn et al., in press). To attract and observe butterflies, people have to provide host and nectar plants for them (Hammer, 2015), which can add beauty to any yard (Vickery, 1995). Education and experience leads to understanding that leads to empowerment which leads to change (Miller, 2005); hopefully a generational paradigm 
shift will occur leading to the coexistence between humans and south Florida's rich biodiverse flora and fauna (Alonso and Heinen, 2011).

\section{LIMITATIONS}

Findings from this study represent only the study participants and may not necessarily be extrapolated to represent the majority of $5^{\text {th }}$ grade students and teachers in MiamiDade County, given the dynamics of cultural diversity and experiences, in conjunction with socioeconomic factors (Moore, 2004; Kurlaender and Yun, 2005). A larger sample size of students (more $5^{\text {th }}$ grade classes) in both groups (experimental and control) would have strengthened representation. Miami-Dade County is a large cosmopolitan metropolis with 197 elementary schools and 44 K-8 centers (Miami-Dade County Public Schools, 2017a,b). An extension of the study to include more students and schools would provide more opportunities to elucidate the effects on place-based education and the intervention (native plant home assignment). Logistical and financial constraints involving time commitment, plant resources, and identification guides increases as the sample size scales up; however, detailed preparation and experience can alleviate some of the constraints through timed plant cultivation, collaboration with nurseries, and dedicated commitment by the teachers at each school including reliable support by the scientific community.

\section{LITERATURE CITED}

Allen, W. 2003. Plant blindness. Bioscience 53(10): 926.

Alonso, J., and Heinen, J.T. 2011. Miami Dade County's environmentally endangered lands program: Local efforts for a global cause. Natural Areas Journal 31(2): 183189. 
Andrzejewski, R., Babińska-Werka, J., Gliwicz, J., and Goszczyński, J. 1978.

Synurbization processes in population of Apodemus agrarius. I. Characteristics of populations in an urbanization gradient. Acta Theriologica, 23(20): 341-358.

Bailey, R. 2006. Physical education and sport in schools: A review of benefits and outcomes. Journal of School Health, 76(8): 397-401.

Balick, M.J., and Cox, P.A. 1996. Plants, people, and culture: The science of ethnobotany. New York: Scientific American Library.

Barnosky, A.D., Matzke, N., Tomiya, S., Wogan, G.O.U., Swartz, B., Quental, T.B., Marshall, C., McGuire, J.L., Lindsey, E.L., Maguire, K.C., Mersey, B., and Ferrer, E.A. 2011. Has the Earth's sixth mass extinction already arrived? Nature, 471: 51-57.

Barriga, P.A., Sternberg, E.D., Lefèvre, T., de Roode, J.C., and Altizer, S. 2016. Occurrence and host specificity of a neogregarine protozoan in four milkweed butterfly hosts (Danaus spp.). Journal of Invertebrate Pathology, 140: 75-82.

Basile, C., and White, C. 2000. Respecting living things: Environmental literacy for young children. Early Childhood Education Journal, 28(1): 57-61.

Bibb, K., and Hughes, P. 2007. United States Fish and Wildlife Service Schaus Swallowtail butterfly (Heraclides aristodemus ponceanus) 5-Year review: Summary and evaluation. United States Fish and Wildlife Service, Southeast Region, South Florida Ecological Services Office, Vero Beach, Florida. Available online at http://ecos.fws.gov/docs/five_year_review/doc1983.pdf. Accessed 15 April 2017.

Boyatzis, R.E. 1998. Transforming qualitative information: Thematic analysis and code development. Sage Publications, Inc., Thousand Oaks, California.

Braun, V., and Clarke, V. 2006. Using thematic analysis in psychology. Qualitative Research in Psychology, 3(2): 77-101.

Brewer, C. 2002. Conservation education partnerships in schoolyard laboratories: A call back to action. Conservation Biology, 16(3): 577-579.

Broom, C. 2017. Exploring the relations between childhood experiences in nature and young adults' environmental attitudes and behaviours. Australian Journal of Environmental Education, 33(1): 34-47.

Brown Jr., K.S., and Freitas, A.V.L. 2002. Butterfly communities of urban forest fragments in Campinas, São Paulo, Brazil: Structure, instability, environmental correlates, and conservation. Journal of Insect Conservation, 6: 217-231. 
Caro, T., Mulder, M.B., and Moore, M. 2003. Effects of conservation education on reasons to conserve biological diversity. Biological Conservation, 114(1): 143-152.

Carpenter, S. K., Pashler, H., Wixted, J.T., and Vul, E. 2008. The effects of tests on learning and forgetting. Memory \& Cognition, 36(2): 438-448.

Cheng, J.C.H., and Monroe, M.C. 2012. Connection to nature: Children's affective attitude toward nature. Environment and Behavior, 44(1): 31-49.

Clayborn, J., Koptur, S., O’Brien, G., and Whelan, K.R.T. 2017. The Schaus swallowtail habitat enhancement project: An applied service-learning project continuum from Biscayne National Park to Miami-Dade County public schools. Southeastern Naturalist, 16(10): 26-46.

Cook, T.D., and Campbell, D.T. 1979. Quasi-experimentation: Design \& analysis issues for field settings. Houghton Mifflin, Boston.

Crutzen, P.J. 2002. Geology of mankind. Nature, 415: 23.

Culin, J. 2002. Butterflies are great teachers: The South Carolina Butterfly Project. American Entomologist, 48(1): 14-18.

Cutting, B.T., and Tallamy, D.W. 2015. An evaluation of butterfly gardens for restoring habitat for the monarch butterfly (Lepidoptera: Danaidae). Environmental entomology, 44(5): 1328-1335.

Daniels, J. 2013. Gardening and landscape modification: Butterfly gardens. In The Management of insects in recreation and tourism (ed.) Lemelin, R.H. Cambridge University Press. Cambridge, England.

Dennis, R.L., Hodgson, J.G., Grenyer, R., Shreeve, T.G., and Roy, D.B. 2004. Host plants and butterfly biology. Do host-plant strategies drive butterfly status? Ecological Entomology, 29(1): 12-26.

Dimitrov, D.M., and Rumrill Jr, P.D. 2003. Pretest-posttest designs and measurement of change. Work, 20(2): 159-165.

Dirzo, R., Young, H.S., Galetti, M., Ceballos, G., Isaac, N.J.B., and Collen, B. 2014. Defaunation in the Anthropocene. Science 345: 401-406.

Feinsinger, P., Margutti, L., and Oviedo, R. 1997. Schoolyard and nature trails: Ecology education outside the university. Trends in Ecology and Evolution, 12: 115-120. 
Fisher-Maltese, C. 2016. "We won't hurt you butterfly!" Second-graders become environmental stewards from experiences in a school garden. International Journal of Early Childhood, 4(1): 55.

FWS (United States Fish and Wildlife Service). 2017. Environmental Conservation Online System (Schaus Swallowtail Butterfly). Available online at http://ecos.fws.gov/speciesProfile/profile/speciesProfile?spcode=I016. Accessed 15 April 2017.

Grajal, A., Luebke, J.F., Kelly, L.D., Matiasek, J., Clayton, S., Karazsia, B.T., Saunders, C.D., Goldman, S.R., Mann, M.E., and Stanoss, R. 2017. The complex relationship between personal sense of connection to animals and self-reported proenvironmental behaviors by zoo visitors. Conservation Biology, 31(2): 322-330.

Grunova, M., Brandlova, K., Svitalek, J., and Hejcmanova, P. 2017. Environmental education supports conservation action by increasing the immediate and long-term environmental knowledge of children in West Africa. Applied Environmental Education and Communication, 16(1): 3-16.

Guiney, M.S., and Oberhauser, K.S. 2009. Insects as flagship conservation species. Terrestrial Arthropod Reviews, 1(2): 111-123.

Hammer, R.L. 2012. Wildflowers of Southeast Florida including The Florida Keys and Everglades National Park: A Guide to Common Native Species. Quick Reference Publishing, Inc., Austin, Texas.

Hammer, R.L. 2015. Attracting hummingbirds and butterflies in tropical Florida: A companion for gardeners. University Press of Florida, Florida.

Hazen, R.M., Grew, E.S., Origlieri, M.J., and Downs, R.T. 2017. On the mineralogy of the "Anthropocene Epoch". American Mineralogist, 102(3): 595-611.

Hoekstra, J.M., Boucher, T.M., Ricketts, T.H., and Roberts, C. 2005. Confronting a biome crisis: Global disparities of habitat loss and protection. Ecology letters 8(1): 23-29.

Howard, E., and Davis, A.K. 2009. The fall migration flyways of monarch butterflies in eastern North America revealed by citizen scientists. Journal of Insect Conservation, 13(3): 279-286.

Jacobi-Vessels, J.L. 2013. Discovering nature: The benefits of teaching outside of the classroom. Dimensions of Early Childhood, 41(3): 4-10.

Jameson, A. 2002. Host Plants and Habitats of the Schaus Swallowtail Butterfly (Papilio Aristodemus Ponceanus). M.S. Thesis. University of Miami, Coral Gables, FL. 51 pp. 
Kazempour, M. 2014. I Can't Teach Science! A Case Study of an Elementary Pre-Service Teacher's Intersection of Science Experiences, Beliefs, Attitude, and Self-Efficacy. International Journal of Environmental and Science Education, 9(1): 77-96.

Klemmer, C.D., Waliczek, T.M., and Zajicek, J.M. 2005. Growing minds: The effect of a school gardening program on the science achievement of elementary students. HortTechnology, 15(3): 448-452.

Knaggs, C.M., and Sondergeld, T.A. 2015. Science as a Learner and as a Teacher: Measuring Science Self-Efficacy of Elementary Preservice Teachers. School Science and Mathematics, 115(3): 117-128.

Koh, L.P., and Sodhi, N.S. 2004. Importance of reserves, fragments, and parks for butterfly conservation in a tropical urban landscape. Ecological Applications, 14(6): 1695-1708.

Koi, S., and Daniels, J. 2015. New and revised life history of the Florida hairstreak Eumaeus atala (Lepidoptera: Lycaenidae) with notes on its current conservation status. Florida Entomologist, 98(4): 1134-1147.

Kurlaender, M., and Yun, J.T. 2005. Fifty Years after Brown: New Evidence of the Impact of School Racial Composition on Student Outcomes. International Journal of Educational Policy, Research, and Practice, 6(1): 51-78.

Kwan, B.K.Y., Cheung, J.H.Y., Law, A.C.K., Cheung, S.G., and Shin, P.K.S. 2017. Conservation education program for threatened Asian horseshoe crabs: A step towards reducing community apathy to environmental conservation. Journal of Nature Conservation, 35: 53-65.

Lemieux, C.M., and Allen, P.D. 2007. Service learning in social work education: The state of knowledge, pedagogical practicalities, and practice conundrums. Journal of Social Work Education, 43(2): 309-325.

Lewis, S.L., and Maslin, M.A. 2015. Defining the Anthropocene. Nature, 519: 171-180.

Libman, K. 2007. Growing youth growing food: How vegetable gardening influences young people's food consciousness and eating habits. Applied Environmental Education and Communication, 6: 87-95.

Lieberman, G.A. and Hoody, L.L. 1998. Closing the Achievement Gap: Using the Environment as an Integrating Context for Learning. San Diego, CA: State Environment and Education Roundtable.

Likert, R. 1932. A technique for the measurement of attitudes. Archives of Psychology, 22(40): 1-55. 
Mahar, M.T., Murphy, S.K., Rowe, D.A., Golden, J., Shields, A.T., and Raedeke, T.D. 2006. Effects of a classroom-based program on physical activity and on-task behavior. Medicine and Science in Sports and Exercise, 38(12): 2086-2094.

Mathew, G., and Anto, M. 2007. In situ conservation of butterflies through establishment of butterfly gardens: A case study at Peechi, Kerala, India. Current science 93(3): 337-347.

Matthews, R.W., Flage, L.R., and Matthews, J.R. 1997. Insects as teaching tools in primary and secondary education. Annual review of entomology, 42(1): 269-289.

McElderry, R.M., Salvato, M.H., and Horvitz, C.C. 2015. Population viability models for an endangered endemic subtropical butterfly: Effects of density and fire on population dynamics and risk of extinction. Biodiversity and conservation, 24(7): 1589-1608.

McIntyre, N.E., Knowles-Yánez, K., and Hope, D. 2008. Urban ecology as an interdisciplinary field: Differences in the use of "urban" between the social and natural sciences. Urban Ecology, 4: 49-65.

Miami-Dade County Public Schools. Accessed 16 April 2017a. Retrieved from http://www.dadeschools.net/schools/schoolinformation/default.asp?type=1\#list

Miami-Dade County Public Schools. Accessed 16 April 2017b. Retrieved from http://www.dadeschools.net/schools/schoolinformation/?type=6\#list

Miller, J.R. 2005. Biodiversity conservation and the extinction of experience. Trends in Ecology and Evolution 20(8): 430-434.

Minno, M.C. 2014. Butterflies of Southeast Florida: A Guide to Common and Notable Species. Quick Reference Publishing, Inc., Boynton Beach, Florida.

Minno, M.C., and Emmel, T.C. 1993. Butterflies of the Florida Keys. Gainesville, Scientific Publishers.

MonarchWatch.org. 2017. Available online at www.monarchwatch.org. Accessed 29 July 2017.

Moore, J.R. 2004. 50 Years after Brown: Segregation in the Miami-Dade County Public Schools. Equity \& Excellence in Education, 37(3): 289-301.

Muñoz, M.A., Scoskie, J.R., and French, D.L. 2013. Investigating the "black box" of effective teaching: The relationship between teachers' perception and student achievement in a large urban district. Educational Assessment, Evaluation and Accountability, 25(3): 205-230. 
Nowak D.J., and Dwyer J.F. 2007. Understanding the Benefits and Costs of Urban Forest Ecosystems. Pp. 25-46. In: Kuser J.E. (eds) Urban and Community Forestry in the Northeast. Springer, Dordrecht.

Obboko, M., and Hannington, A. 2014. An assessment of the effectiveness of school gardens in church founded primary schools in Pachwa Sub-County, Kibaale District. International Academic Journals, 1(1): 97-103.

Oliver, T., Roy, D.B., Hill, J.K., Brereton, T. and Thomas, C.D. 2010. Heterogeneous landscapes promote population stability. Ecology letters, 13: 473-84.

Olivier, T., Schmucki, R., Fontaine, B., Villemey, A., and Archaux, F. 2016. Butterfly assemblages in residential gardens are driven by species' habitat preference and mobility. Landscape Ecology, 31(4): 865-876.

Padgett, D.K. 2008. Qualitative Methods in Social Work Research, Second Edition. Sage Publications, Inc., Thousand Oaks, California.

Pe'er, G. and Settele, J. 2008. Butterflies in and for conservation: Trends and prospects. Israel Journal of Ecology and Evolution 54: 7-17.

Pyle, R. 2002. Eden in a vacant lot: Special places, species and kids in community of life. In: Children and Nature: Psychological, Sociocultural and Evolutionary Investigations. Kahn, P.H. and Kellert, S.R. (eds) Cambridge: MIT Press pp. 305-327.

Quade, D. 1967. Rank analysis of covariance. Journal of the American Statistical Association, 62(320): 1187-1200.

Rader, R., Bartomeus, I., Garibaldi, L.A., Garratt, M.P., Howlett, B.G., Winfree, R., Bommarco, R., Brittain, C., Carvalheiro, L.G., Chacoff, N.P., Entling, M.H., Foully, B., Freitas, B.M., Gemmill-Herren, B., Ghazoul, J., Griffin, S.R., Gross, C.L., Herbertsson, L., Herzog F., Hipólito, J., Jaggara, S., Jauker, F., Kleinz, A., Kleijn, D., Krishnan, S., Lemos, C.Q., Lindström, S.A.M., Mandelik, Y., Monteiro, V.M., Nelson, W., Nilsson, L., Pattemore, D.E., Pereira, N.O., Pisanty, G., Potts, S.G., Reemer, M., Rundlöf, M., Sheffield, C.S., Scheper, J., Schüepp, C., Smith, H.G., Stanley, D.A., Stout, J.C., Szentgyörgyi, H., Taki, H., Vergara, C.H., Viana, B.F., and Woyciechowski, M. 2016. Non-bee insects are important contributors to global crop pollination. Proceedings of the National Academy of Sciences, 113(1): 146-151.

Ramírez-Restrepo, L., Koi, S., and MacGregor-Fors, I. 2017. Tales of urban conservation: Eumaeus butterflies and their threatened cycad hostplants. Urban Ecosystems, 20(2): 375-378.

Reichard S.H., and White P. 2001. Horticulture as a pathway of invasive plant introductions in the United States. BioScience, 51: 103-113. 
Revathy, V.S., Mathew, G., and Narayanankutty, T.P. 2014. Role of recreated habitats in butterfly conservation: A case study at Thenmala, Kerala, India. International Journal of Tropical Insect Science, 34(4): 287-295.

Ricketts, T. 2001. The matrix matters: Effective isolation in fragmented landscapes. The American Naturalist, 158: 87-99.

Riggs, I., and Enochs, L. 1990. Towards the development of an elementary teacher's science teaching efficacy belief instrument. Science Education, 74: 625-637.

Ross, M.S., O'Brien, J.J., and Flynn, L.J. 1992. Ecological site classification of Florida Keys terrestrial habitats. Biotropica, 24(4): 488-502.

Rudd, H., Vala, J., and Schaefer, V. 2002. Importance of backyard habitat in a comprehensive biodiversity conservation strategy: A connectivity analysis of urban green spaces. Restoration ecology, 10(2): 368-375.

Salvato, M.H. 2003. Butterfly conservation and host plant fluctuations: The relationship between Strymon acis bartrami and Anaea troglodyta floridalis on Croton linearis in Florida (Lepidoptera: Lycaenidae and Nymphalidae). Holarctic Lepidoptera, 10(1-2): 53-57.

Satterfield, D.A., Maerz, J.C., and Altizer, S. 2015. Loss of migratory behaviour increases infection risk for a butterfly host. Proceedings of the Royal Society BBiological Sciences, 282(1801): 20141734.

Savard, J.P.L., Clergeau, P., and Mennechez, G. 2000. Biodiversity concepts and urban ecosystems. Landscape and urban planning, 48(3): 131-142.

Schultz, C.B., and Dlugosch, K.M. 1999. Nectar and hostplant scarcity limit populations of an endangered Oregon butterfly. Oecologia, 119(2): 231-238.

Settlage, J., and Southerland, S.A. 2007. Teaching Science to every child: Using culture as a starting point. Routledge, Taylor \& Francis Group, New York.

Skelly, S.M., and Bradley, J.C. 2000. The importance of school gardens as perceived by Florida elementary school teachers. HortTechnology, 10(1): 229-231.

Smith, D.S., Miller, L.D., Miller, J.Y., and Lewington, R. 1994. The butterflies of the West Indies and south Florida. Oxford University Press, New York.

Smith, E. 2002. The effects of season, host plant protection, and ant predators on the survival of Eumaeus atala (Lycaenidae) in re-establishments. Journal of the Lepidopterists' Society, 56(4): 272-276. 
Snyder, J.R., Herndon, A. and Robertson, W.B., Jr. 1990. South Florida rockland. In: Myers, R.L. \& Ewel, J.J. (eds) Ecosystems of Florida. University of Central Florida Press, Orlando, FL, pp. 230-277.

Sobel, D. 2005. Place-Based Education: Connecting Classrooms \& Communities. The Orion Society, Great Barrington, MA.

Solter, L.F. 1997. Is entomological research child's play? Teaching children scientific methods. American Entomologist, 43(4):198-200.

Stronge, J. 2013. Effective teachers = student achievement: What the research says. Routledge, New York.

Supovitz, J.A., and Turner, H.M. 2000. The effects of professional development on science teaching practices and classroom culture. Journal of Research in Science Teaching, 37(9):963-980.

Taki, H., and Kevan, P.G. 2007. Does habitat loss affect the communities of plants and insects equally in plant-pollinator interactions? Preliminary findings. Biodiversity and Conservation, 16(11): 3147-3161.

Tashakkori, A., and Teddlie, C. 1998. Mixed methodology: Combining qualitative and quantitative approaches. Sage, Vol. 46, Thousand Oaks, California.

Tatarchuk, S., and Eick, C. 2011. Outdoor integration: Looking to nature to accentuate science and language arts connections. Science and Children, 48(6): 35-39.

Vickery, M.L. 1995. Gardens: the neglected habitat. Pp. 123-134, In A.S. Pullin (Ed.). Ecology and conservation of butterflies. Chapman and Hall, Netherlands.

Wagner, D.L., and Van Driesche, R.G. 2010. Threats posed to rare or endangered insects by invasions of nonnative species. Annual Review of Entomology, 55: 547-568.

Waliczek, T.M., and Zajicek, J.M. 1999. School gardening: Improving environmental attitudes of children through hands-on learning. Journal of Environmental Horticulture 17: 180-184.

Wandersee, J.H., and Schussler, E.E. 1999. Preventing plant blindness. National Association of Biology Teachers, 61(2): 84-86.

Wells, N.M., and Lekies, K.S. 2006. Nature and the life course: Pathways from childhood nature experiences to adult environmentalism. Children, Youth and Environments, 16(1): 1-24. 
Whelan, K.R. 2011. Schaus Swallowtail butterfly (Heraclides aristodemus ponceanus) habitat enhancement in Biscayne National Park. Project Report. Funded by FWS South Florida Coastal Program. National Park Service, Palmetto Bay, FL.

Whelan, K.R.T., and Atkinson, A. 2015. Schaus Swallowtail Butterfly (Heraclides aristodemus ponceanus) Habitat Enhancement in Biscayne National Park. South Florida Coastal Program Project Progress Report. NPS/SFCN. National Park Service, Palmetto Bay, Florida. 


\section{TABLES}

Table 4.1: Survey statements and interview questions used during the study. All students $(\mathrm{n}=120)$ at each school (Air Base K-8

Center, North Hialeah Elementary, Whispering Pines Elementary) and treatment (experimental and control groups) were

administered pre- and post-survey statements. A total of 48 students (16 students at each school; however, 8 students for each treatment) participated in pre- and post-interviews.

\begin{tabular}{|l|}
\hline \multicolumn{1}{|c|}{ Survey Statement } \\
\hline 1) I like to learn about animals. \\
\hline 2) I like to learn about insects. \\
\hline 3) I like to learn about plants. \\
\hline 4) I like to learn about the environment \\
\hline 5) Insects are important to the environment. \\
\hline 6) Plants are important to the environment. \\
\hline 7) Animals are important to the environment. \\
\hline 8) Animals are easily harmed by people. \\
\hline 9) Plants are easily harmed by people. \\
\hline 10) Insects are easily harmed by people. \\
\hline 11) The environment is easily harmed by people. \\
\hline 12) I would give some of my money to help save animals. \\
\hline 13) I would give some of my money to help save insects. \\
\hline 14) I would give some of my money to help save plants and trees. \\
\hline 15) I would give some of my money to help save butterflies. \\
\hline 16) I would give some of my money to help save bees. \\
\hline
\end{tabular}


17) I like to spend time in places that have bees.

18) I like to spend time in places that have plants.

19) I like to spend time in places that have animals.

20) I like to spend time in places that have insects.

21) I like to spend time in places that have butterflies.

22) It makes me sad to see buildings and homes where plants and animals used to be.

23) I would like to help clean up the environment in my neighborhood.

24) People need animals to live.

25) People need plants and trees to live.

26) People need insects to live.

27) People need butterflies to live.

28) People need bees to live.

1) What kind of activities do you enjoy doing outdoors?

2) What kind of activities do you like to do indoors?

3) How much time do you spend outside during school?

4) How much time do you spend outside after school?

5) Can you describe a butterfly garden?

6) Name some things you need to attract butterflies to a garden?

7) Do you have a garden outside of school?

8) (If yes to \#7) Tell me about some of the plants in your garden.

9) Would it be easy or difficult for animals and plants to survive in your neighborhood, briefly explain?

10) Describe some reasons butterflies and other insects are disappearing, going extinct.

11) Would you recommend a butterfly garden to a friend, can you explain? 
Table 4.2: Plants planted in the school butterfly gardens. Overall, 18-19 native plant species were distributed to Air Base K-8 Center (AB), North Hialeah Elementary (NHE), and Whispering Pines Elementary (WPE). Number of plants are reported below each school. The last column on the right depicts showy butterflies, moths, and skippers attracted to host plants planted in the school gardens (there are other less showy moth species, not listed). Some butterfly, moth, and skipper species are imperiled and unlikely to visit the school gardens; however, each plant added structural and functional value to the native plant butterfly garden.

\begin{tabular}{|l|c|c|c|c|l|}
\hline \multicolumn{1}{|c|}{ Plant Species } & AB & NHE & WPE & Total & $\begin{array}{l}\text { Butterfly, moth, and skipper species that use the plant as a host } \\
\text { (caterpillars eat the leaves) }\end{array}$ \\
\hline Angadenia berteroi & 8 & 6 & 6 & 20 & Syntomeida epilais \\
\hline Asclepias verticillata & 4 & 4 & 4 & 12 & Danaus gilippus, Danaus plexippus \\
\hline Asclepias tuberosa & 1 & 1 & 1 & 3 & Danaus gilippus, Danaus plexippus \\
\hline Bourreria succulenta & 3 & 2 & 2 & 7 & \\
\hline Byrsonima lucida & 3 & 2 & 2 & 7 & *Ephyriades brunneus \\
\hline Chamaecrista fasciculata & 4 & 4 & 4 & 12 & Eurema lisa, Eurema nicippe, Hemiargus ceraunus, Phoebis sennae \\
\hline Colubrina elliptica & 5 & 5 & 5 & 15 & \\
\hline Croton linearis & 2 & 2 & 2 & 6 & *Anaea troglodyte floridalis, *Strymon acis bartrami \\
\hline Cynophalla flexuosa & 1 & 1 & 1 & 3 & $*$ *Appias drusilla, Ascia monuste \\
\hline Guapira discolor & 3 & 3 & 3 & 9 & \\
\hline Lantana involucrata & 4 & 2 & 2 & 8 & \\
\hline Passiflora suberosa & 6 & 4 & 5 & 15 & Agraulis vanilla, Dryas iulia, Heliconius charitonius \\
\hline Pithecellobium keyense & 2 & 2 & 2 & 6 & Leptotes cassius, Phoebis agarithe \\
\hline Plumbago zeylanica & 0 & 3 & 0 & 3 & Leptotes cassius \\
\hline Psychotria nervosa & 2 & 1 & 3 & 6 & \\
\hline Salvia coccinea & 3 & 2 & 3 & 8 & \\
\hline
\end{tabular}




\begin{tabular}{|l|c|c|c|c|l|}
\hline Senna mexicana & 2 & 8 & 2 & 12 & Phoebis philea, Phoebis sennae \\
\hline Zamia integrifolia & 3 & 2 & 4 & 9 & Eumaeus atala \\
\hline Zanthoxylum fagara & 3 & 2 & 2 & 7 & $\begin{array}{l}\text { *Heraclides aristodemus ponceanus, *Heraclides andraemon, } \\
\text { Heraclides cresphontes }\end{array}$ \\
\hline Total & 59 & 56 & 53 & 168 & *imperiled \\
\hline
\end{tabular}


Table 4.3: Science Teaching Efficacy Belief Instrument results from each school $(\mathrm{AB}=$ Air Base K-8 Center, NHE $=$ North

Hialeah Elementary, WPE = Whispering Pines Elementary; Riggs and Enochs, 1990). SA = Strongly Agree, A = Agree, UN =

Uncertain, D = Disagree, SD = Strongly Disagree. Results from 3 individual science teachers, one at each school.

\begin{tabular}{|c|c|c|c|}
\hline Statement & AB & NHE & WPE \\
\hline 1) When a student does better than usual in science, it is often because the teacher exerted a little extra effort. & $\mathrm{D}$ & A & A \\
\hline 2) I am continually finding better ways to teach science. & SA & A & UN \\
\hline 3) Even when I try very hard, I don't teach science as well as I do most subjects. & A & $\mathrm{D}$ & A \\
\hline $\begin{array}{l}\text { 4) When the science grades of students improve, it is most often due to their teachers having found a more } \\
\text { effective teaching approach. }\end{array}$ & $\mathrm{UN}$ & A & SA \\
\hline 5) I know the steps necessary to teach science concepts effectively. & $\mathrm{D}$ & UN & A \\
\hline 6) I am not very effective in monitoring science experiments. & $\mathrm{D}$ & $\mathrm{D}$ & A \\
\hline 7) If students are underachieving in science, it is most likely due to ineffective science teaching. & $\mathrm{D}$ & $\mathrm{D}$ & $\mathrm{D}$ \\
\hline 8) I generally teach science ineffectively. & $\mathrm{D}$ & $\mathrm{D}$ & $\mathrm{D}$ \\
\hline 9) The inadequacy of a student's science background can be overcome by good teaching. & A & A & UN \\
\hline 10) The low science achievement of some students cannot generally be blamed on their teachers. & SA & A & A \\
\hline 11) When a low achieving child progresses in science, it is usually due to extra attention given by the teacher. & A & A & A \\
\hline 12)I understand science concepts well enough to be effective in teaching elementary science. & UN & A & A \\
\hline 13) Increased effort in science teaching produces little change in some students' science achievement. & $\mathrm{D}$ & $\mathrm{D}$ & $\mathrm{D}$ \\
\hline 14) The teacher is generally responsible for the achievement of students in science. & $\mathrm{UN}$ & A & $\mathrm{D}$ \\
\hline 15) Students' achievement in science is directly related to their teacher's effectiveness in science teaching. & UN & $\mathrm{D}$ & $\mathrm{D}$ \\
\hline $\begin{array}{l}\text { 16) If parents comment that their child is showing more interest in science at school, it is probably due to the } \\
\text { performance of the child's teacher. }\end{array}$ & A & A & A \\
\hline 17) I find it difficult to explain to students' why science experiments work. & SD & $\mathrm{D}$ & $\mathrm{D}$ \\
\hline 18) I am typically able to answer students' science questions. & $\mathrm{A}$ & A & A \\
\hline 19) I wonder if I have the necessary skills to teach science. & SD & $\mathrm{D}$ & $\mathrm{D}$ \\
\hline
\end{tabular}




\begin{tabular}{|c|c|c|c|}
\hline 20) Effectiveness in science teaching has little influence on the achievement of students with low motivation. & $\mathrm{D}$ & $\mathrm{D}$ & $\mathrm{D}$ \\
\hline 21) Given a choice, I would not invite the principal to evaluate my science teaching. & SD & $\mathrm{D}$ & UN \\
\hline $\begin{array}{l}\text { 22) When a student has difficulty understanding a science concept, I am usually at a loss as to how to help the } \\
\text { student understand it better. }\end{array}$ & SD & $\mathrm{D}$ & $\mathrm{D}$ \\
\hline 23) When teaching science, I usually welcome student questions. & SA & A & SA \\
\hline 24) I don't know what to do to turn students on to science. & SD & $\mathrm{D}$ & $\mathrm{D}$ \\
\hline 25) Even teachers with good science teaching abilities cannot help some kids learn science. & SD & A & UN \\
\hline
\end{tabular}


Table 4.4: Summary statistics of post-test scores (dependent variable) using an Analysis of Covariance (ANCOVA). The pre-test score was the covariate with the intervention as the independent variable. The intervention had a significant effect on post-test scores. Students in the experimental group significantly scored higher on the post-test in contrast to students in the control group (see Figures 4-7). Asterisk denotes significance.

\begin{tabular}{|c|c|c|c|c|c|}
\hline Source & Sum of Squares & df & Mean Square & $\mathbf{F}$ & Significance \\
\hline \multicolumn{6}{|l|}{ All Schools } \\
\hline Intercept & 0.918 & 1 & 0.918 & 57.985 & $0.001^{*}$ \\
\hline Pre-test & 0.870 & 1 & 0.870 & 54.953 & $0.001^{*}$ \\
\hline Intervention & 0.408 & 1 & 0.408 & 25.771 & $0.001 *$ \\
\hline Error & 1.853 & 117 & 0.016 & & \\
\hline \multicolumn{6}{|c|}{ Air Base K-8 Center } \\
\hline Intercept & 0.360 & 1 & 0.360 & 43.615 & $0.001 *$ \\
\hline Pre-test & 0.001 & 1 & 0.001 & 0.026 & 0.873 \\
\hline Intervention & 0.082 & 1 & 0.082 & 9.926 & $0.003 *$ \\
\hline Error & 0.314 & 38 & 0.008 & & \\
\hline \multicolumn{6}{|c|}{ North Hialeah Elementary } \\
\hline Intercept & 0.209 & 1 & 0.209 & 9.089 & $0.005^{*}$ \\
\hline Pre-test & 0.145 & 1 & 0.145 & 6.304 & $0.017 *$ \\
\hline Intervention & 0.143 & 1 & 0.143 & 6.203 & $0.018 *$ \\
\hline Error & 0.804 & 35 & 0.230 & & \\
\hline \multicolumn{6}{|c|}{ Whispering Pines Elementary } \\
\hline Intercept & 0.440 & 1 & 0.440 & 31.052 & $0.001^{*}$ \\
\hline Pre-test & 0.297 & 1 & 0.297 & 20.976 & $0.001^{*}$ \\
\hline Intervention & 0.156 & 1 & 0.156 & 10.986 & $0.002 *$ \\
\hline Error & 0.539 & 38 & 0.014 & & \\
\hline
\end{tabular}


Table 4.5: A total of $1205^{\text {th }}$ grade students attending three schools completed pre- and post-surveys. Sixty students were in experimental groups while the other 60 were in control groups. An exploratory factor analysis was performed and reduced 28 survey statements into 14 survey statements that loaded into 5 factors.

Quade's rank analysis of covariance was used to analyze survey results. Post-survey score was the dependent variable, pre-survey score was the covariate with the influence of gardens present at the place of residence before the study commenced, intervention of taking host and nectar plants home, and type of students' residence as independent variables. Type of residence was tested for all schools; however, it was not tested for individual schools due to small sample size.

\begin{tabular}{|c|c|c|c|c|c|c|}
\hline \multicolumn{7}{|c|}{ Factor 1 - Stewardship } \\
\hline$\# 12$ & \multicolumn{6}{|c|}{ I would give some of my money to help save animals. } \\
\hline$\# 14$ & \multicolumn{6}{|c|}{ I would give some of my money to help save plants and trees. } \\
\hline$\# 23$ & \multicolumn{6}{|c|}{ I would like to help clean up the environment in my neighborhood. } \\
\hline \multicolumn{7}{|c|}{ Factor 2 - Learning Interest (Nature) } \\
\hline$\# 1$ & \multicolumn{6}{|c|}{ I like to learn about animals. } \\
\hline \#3 & \multicolumn{6}{|c|}{ I like to learn about plants. } \\
\hline$\# 4$ & \multicolumn{6}{|c|}{ I like to learn about the environment. } \\
\hline \multicolumn{7}{|c|}{ Factor 3 - Insects } \\
\hline$\# 2$ & \multicolumn{6}{|c|}{ I like to learn about insects. } \\
\hline \#20 & \multicolumn{6}{|c|}{ I like to spend time in places that have insects. } \\
\hline \multicolumn{7}{|c|}{ Factor 4 - Interconnectedness (Bee and Plants) } \\
\hline \#6 & \multicolumn{6}{|c|}{ Plants are important to the environment. } \\
\hline$\# 28$ & \multicolumn{6}{|c|}{ People need bees to live. } \\
\hline \multicolumn{7}{|c|}{ Factor 5 - Anthropogenic Harm } \\
\hline$\# 8$ & \multicolumn{6}{|c|}{ Animals are easily harmed by people. } \\
\hline \#9 & \multicolumn{6}{|c|}{ Plants are easily harmed by people. } \\
\hline$\# 11$ & \multicolumn{6}{|c|}{ The environment is easily harmed by people. } \\
\hline \multicolumn{7}{|c|}{ Schools } \\
\hline \multicolumn{2}{|r|}{ Source } & $\begin{array}{l}\text { Sum of } \\
\text { Squares }\end{array}$ & df & Mean Square & $\mathbf{F}$ & Significance \\
\hline
\end{tabular}




\begin{tabular}{|c|c|c|c|c|c|}
\hline \multicolumn{6}{|l|}{ Stewardship } \\
\hline Intervention & 451.077 & 1 & 451.077 & 0.384 & 0.537 \\
\hline Residence & 3628.042 & 1 & 3628.042 & 1.568 & 0.213 \\
\hline Garden at Residence & 103.698 & 1 & 103.698 & 0.088 & 0.767 \\
\hline \multicolumn{6}{|c|}{ Learning Interest (Nature) } \\
\hline Intervention & 23.124 & 1 & 23.124 & 0.019 & 0.889 \\
\hline Residence & 418.668 & 1 & 418.668 & 0.175 & 0.840 \\
\hline Garden at Residence & 33.724 & 1 & 33.724 & 0.028 & 0.867 \\
\hline \multicolumn{6}{|l|}{ Insects } \\
\hline Intervention & 1048.373 & 1 & 1048.373 & 0.889 & 0.348 \\
\hline Residence & 68.363 & 1 & 68.363 & 0.029 & 0.972 \\
\hline Garden at Residence & 735.246 & 1 & 735.246 & 0.622 & 0.432 \\
\hline \multicolumn{6}{|c|}{ Interconnectedness (Bees and Plants) } \\
\hline Intervention & 1449.649 & 1 & 1449.649 & 1.298 & 0.257 \\
\hline Residence & 2641.917 & 1 & 2641.917 & 1.184 & 0.310 \\
\hline Garden at Residence & 4031.248 & 1 & 4031.248 & 3.683 & 0.057 \\
\hline \multicolumn{6}{|l|}{ Anthropogenic Harm } \\
\hline Intervention & 708.809 & 1 & 708.809 & 0.602 & 0.439 \\
\hline Residence & 3186.678 & 1 & 3186.678 & 1.367 & 0.259 \\
\hline Garden at Residence & 530.940 & 1 & 530.940 & 0.451 & 0.503 \\
\hline \multicolumn{6}{|c|}{$\begin{array}{ll}\text { Air Base K-8 Center } \\
\end{array}$} \\
\hline Source & $\begin{array}{l}\text { Sum of } \\
\text { Squares }\end{array}$ & df & Mean Square & $\mathbf{F}$ & Significance \\
\hline \multicolumn{6}{|l|}{ Stewardship } \\
\hline Intervention & 183.083 & 1 & 183.083 & 1.329 & 0.256 \\
\hline Garden at Residence & 68.882 & 1 & 68.882 & 0.490 & 0.488 \\
\hline \multicolumn{6}{|c|}{ Learning Interest (Nature) } \\
\hline Intervention & 21.712 & 1 & 21.712 & 0.155 & 0.696 \\
\hline Garden at Residence & 5.393 & 1 & 5.393 & 0.038 & 0.846 \\
\hline \multicolumn{6}{|l|}{ Insects } \\
\hline Intervention & 1.092 & 1 & 1.092 & 0.008 & 0.931 \\
\hline Garden at Residence & 42.212 & 1 & 42.212 & 0.298 & 0.588 \\
\hline \multicolumn{6}{|c|}{ Interconnectedness (Bees and Plants) } \\
\hline Intervention & 2.364 & 1 & 2.364 & 0.019 & 0.890 \\
\hline Garden at Residence & 4.995 & 1 & 4.995 & 0.041 & 0.841 \\
\hline \multicolumn{6}{|l|}{ Anthropogenic Harm } \\
\hline Intervention & 93.283 & 1 & 93.283 & 0.694 & 0.410 \\
\hline Garden at Residence & 106.634 & 1 & 106.634 & 0.795 & 0.378 \\
\hline \multicolumn{6}{|c|}{ North Hialeah Elementary } \\
\hline Source & $\begin{array}{l}\text { Sum of } \\
\text { Squares }\end{array}$ & df & Mean Square & $\mathbf{F}$ & Significance \\
\hline
\end{tabular}




\begin{tabular}{|c|c|c|c|c|c|}
\hline Intervention & 9.892 & 1 & 9.892 & 0.079 & 0.780 \\
\hline Garden at Residence & 18.018 & 1 & 18.018 & 0.144 & 0.706 \\
\hline \multicolumn{6}{|c|}{ Learning Interest (Nature) } \\
\hline Intervention & 43.925 & 1 & 43.925 & 0.361 & 0.552 \\
\hline Garden at Residence & 20.354 & 1 & 20.354 & 0.166 & 0.686 \\
\hline \multicolumn{6}{|l|}{ Insects } \\
\hline Intervention & 251.296 & 1 & 251.296 & 2.177 & 0.149 \\
\hline Garden at Residence & 13.323 & 1 & 13.323 & 0.109 & 0.743 \\
\hline \multicolumn{6}{|c|}{ Interconnectedness (Bees and Plants) } \\
\hline Intervention & 289.663 & 1 & 289.663 & 2.468 & 0.125 \\
\hline Garden at Residence & 320.376 & 1 & 320.376 & 2.749 & 0.106 \\
\hline \multicolumn{6}{|l|}{ Anthropogenic Harm } \\
\hline Intervention & 55.336 & 1 & 55.336 & 0.445 & 0.509 \\
\hline Garden at Residence & 14.691 & 1 & 14.691 & 0.117 & 0.734 \\
\hline \multicolumn{6}{|c|}{ Whispering Pine Elementary } \\
\hline Source & $\begin{array}{c}\text { Sum of } \\
\text { Squares }\end{array}$ & df & Mean Square & $\mathbf{F}$ & Significance \\
\hline \multicolumn{6}{|l|}{ Stewardship } \\
\hline Intervention & 6.073 & 1 & 6.073 & 2.146 & 0.151 \\
\hline Garden at Residence & 5.654 & 1 & 5.654 & 1.948 & 0.171 \\
\hline \multicolumn{6}{|c|}{ Learning Interest (Nature) } \\
\hline Intervention & 0.011 & 1 & 0.011 & 0.542 & 0.466 \\
\hline Garden at Residence & 0.012 & 1 & 0.012 & 0.550 & 0.463 \\
\hline \multicolumn{6}{|l|}{ Insects } \\
\hline Intervention & 5.125 & 1 & 5.125 & 2.186 & 0.148 \\
\hline Garden at Residence & 0.079 & 1 & 0.079 & 0.033 & 0.856 \\
\hline \multicolumn{6}{|c|}{ Interconnectedness (Bees and Plants) } \\
\hline Intervention & 6.185 & 1 & 6.185 & 8.213 & $0.007 *$ \\
\hline Garden at Residence & 0.073 & 1 & 0.073 & 0.080 & 0.778 \\
\hline \multicolumn{6}{|l|}{ Anthropogenic Harm } \\
\hline Intervention & 15.608 & 1 & 15.608 & 6.155 & $0.018 *$ \\
\hline Garden at Residence & 0.487 & 1 & 0.487 & 0.167 & 0.686 \\
\hline
\end{tabular}


Table 4.6: Results from the Likert scale pre- and post-surveys. Mean and standard deviations were reported displaying students' responses toward statements loaded in each factor. Bold indicates significant changes in students' attitude (see results in Table 5).

\begin{tabular}{|c|c|c|c|c|c|c|c|c|}
\hline \multicolumn{3}{|c|}{ All Schools } & \multicolumn{2}{|c|}{ Air Base } & \multicolumn{2}{|c|}{ NHE } & \multicolumn{2}{|c|}{ WPE } \\
\hline & Mean & SD & Mean & SD & Mean & SD & Mean & SD \\
\hline \multicolumn{9}{|c|}{ Stewardship } \\
\hline Pre Con & 4.260 & 0.713 & 4.317 & 0.703 & 4.117 & 0.777 & 4.351 & 0.626 \\
\hline Post Con & 4.119 & 0.726 & 4.033 & 0.788 & 4.250 & 0.752 & 4.070 & 0.598 \\
\hline Pre Ex & 4.131 & 0.871 & 4.238 & 0.676 & 4.386 & 0.522 & 3.794 & 1.148 \\
\hline Post Ex & 4.202 & 0.710 & 4.333 & 0.600 & 4.386 & 0.533 & 3.904 & 0.843 \\
\hline \multicolumn{9}{|c|}{ Learning Interest (Nature) } \\
\hline Pre Con & 4.040 & 0.762 & 4.067 & 0.892 & 4.300 & 0.730 & 3.737 & 0.491 \\
\hline Post Con & 4.136 & 0.664 & 4.033 & 0.690 & 4.433 & 0.528 & 3.930 & 0.654 \\
\hline Pre Ex & 4.093 & 0.864 & 4.000 & 0.756 & 4.526 & 0.487 & 3.794 & 1.056 \\
\hline Post Ex & 4.137 & 0.718 & 4.111 & 0.786 & 4.316 & 0.501 & 4.000 & 0.777 \\
\hline \multicolumn{9}{|l|}{ Insects } \\
\hline Pre Con & 3.051 & 1.092 & 3.400 & 1.079 & 3.375 & 1.023 & 2.342 & 0.796 \\
\hline Post Con & 3.331 & 0.977 & 3.300 & 1.030 & 3.65 & 0.808 & 3.026 & 0.980 \\
\hline Pre Ex & 3.139 & 1.083 & 3.476 & 1.229 & 3.211 & 0.922 & 2.738 & 0.921 \\
\hline Post Ex & 3.172 & 0.923 & 3.357 & 0.902 & 3.342 & 0.744 & 2.833 & 0.992 \\
\hline \multicolumn{9}{|c|}{ Interconnectedness (Bees and Plants) } \\
\hline Pre Con & 4.144 & 0.798 & 4.650 & 0.421 & 3.950 & 0.805 & 3.816 & 0.831 \\
\hline Post Con & 4.263 & 0.653 & 4.450 & 0.610 & 4.100 & 0.735 & 4.237 & 0.547 \\
\hline Pre Ex & 4.418 & 0.691 & 4.357 & 0.600 & 4.421 & 0.730 & 4.476 & 0.732 \\
\hline Post Ex & 4.393 & 0.544 & 4.476 & 0.663 & 4.474 & 0.443 & 4.238 & 0.453 \\
\hline \multicolumn{9}{|c|}{ Anthropogenic Harm } \\
\hline Pre Con & 3.983 & 0.824 & 4.200 & 0.653 & 3.367 & 0.888 & 4.404 & 0.453 \\
\hline
\end{tabular}




\begin{tabular}{|c|c|c|c|c|c|c|c|c|}
\hline Post Con & 4.175 & 0.645 & 4.35 & 0.499 & 4.050 & 0.769 & 4.123 & 0.594 \\
\hline Pre Ex & 3.858 & 0.779 & 3.984 & 0.613 & 3.965 & 0.620 & 3.635 & 0.981 \\
\hline Post Ex & 4.186 & 0.861 & 4.095 & 0.880 & 4.298 & 0.657 & 4.175 & 0.985 \\
\hline
\end{tabular}


Table 4.7: Results from pre- and post-interviews. The most prominent themes (> 20\% of respondents) inferred from interviews were displayed as decimals because of unequal respondents between students in the control and experimental groups. There were 24 respondents each (pre- and post-interviews) in the control group; however, 22 respondents conducted pre-interviews and 16 conducted post-interviews in the experimental group.

\begin{tabular}{|c|c|c|c|c|c|c|}
\hline \multicolumn{7}{|c|}{ 1) What kind of activities do you enjoy doing outdoors? } \\
\hline All Schools & & Sports & \multicolumn{2}{|c|}{ Random Play } & \multicolumn{2}{|c|}{ Gardening/Yard Exploration } \\
\hline Pre Con & & 0.667 & \multicolumn{2}{|c|}{0.583} & \multicolumn{2}{|c|}{0.250} \\
\hline Post Con & & 0.625 & \multicolumn{2}{|c|}{0.583} & \multicolumn{2}{|r|}{0.375} \\
\hline Pre Ex & & 0.409 & \multicolumn{2}{|c|}{0.500} & \multicolumn{2}{|r|}{0.500} \\
\hline Post Ex & & 0.500 & \multicolumn{2}{|c|}{0.688} & \multicolumn{2}{|r|}{0.313} \\
\hline \multicolumn{7}{|c|}{ 2) What kind of activities do you like to do indoors? } \\
\hline All Schools & TV & Read/Study & Playing & \multicolumn{2}{|c|}{ Electronics/Computer } & Draw/Arts \& Craft \\
\hline Pre Con & 0.208 & 0.125 & 0.458 & \multicolumn{2}{|c|}{0.417} & 0.292 \\
\hline Post Con & 0.208 & 0.250 & 0.417 & \multicolumn{2}{|c|}{0.292} & 0.167 \\
\hline Pre Ex & 0.455 & ------- & 0.500 & \multicolumn{2}{|c|}{0.273} & 0.227 \\
\hline Post Ex & 0.250 & ------- & 0.563 & \multicolumn{2}{|c|}{0.375} & 0.188 \\
\hline \multicolumn{7}{|c|}{ 3) How much time do you spend outside during school? } \\
\hline All Schools & \multicolumn{2}{|c|}{ Range (minutes) } & \multicolumn{2}{|c|}{ Median (minutes) } & \multicolumn{2}{|c|}{ Mode (minutes) } \\
\hline Pre Con & \multicolumn{2}{|r|}{$15-120$} & \multicolumn{2}{|c|}{45} & \multicolumn{2}{|r|}{45} \\
\hline Post Con & \multicolumn{2}{|r|}{$15-180$} & \multicolumn{2}{|c|}{45} & \multicolumn{2}{|r|}{30} \\
\hline Pre Ex & \multicolumn{2}{|r|}{$10-180$} & \multicolumn{2}{|c|}{60} & \multicolumn{2}{|r|}{60} \\
\hline Post Ex & \multicolumn{2}{|r|}{$15-120$} & \multicolumn{2}{|c|}{52.5} & \multicolumn{2}{|r|}{60} \\
\hline \multicolumn{7}{|c|}{ 4) How much time do you spend outside after school? } \\
\hline All Schools & \multicolumn{2}{|c|}{ Range (minutes) } & \multicolumn{2}{|c|}{ Median (minutes) } & & Iode (minutes) \\
\hline
\end{tabular}




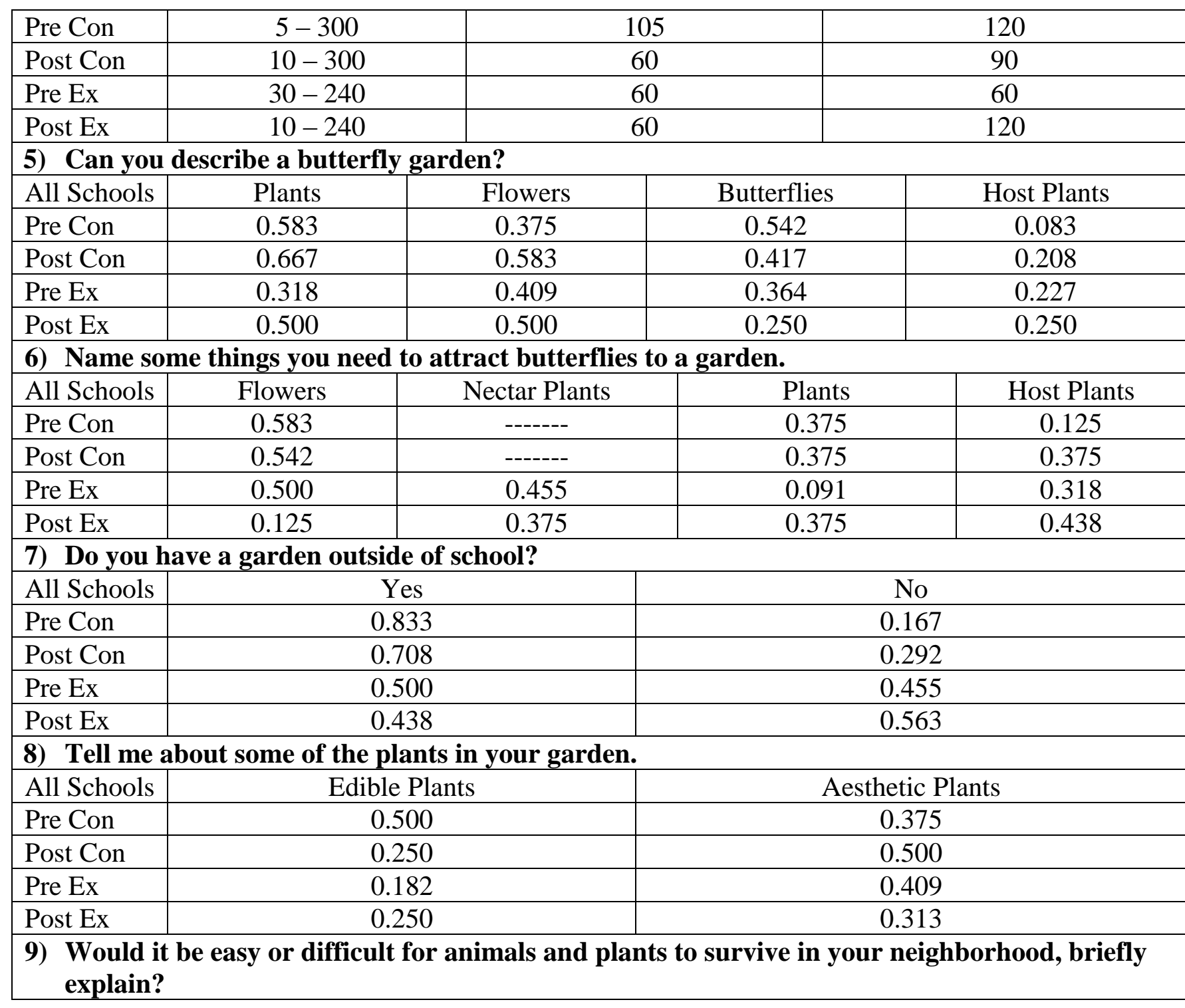




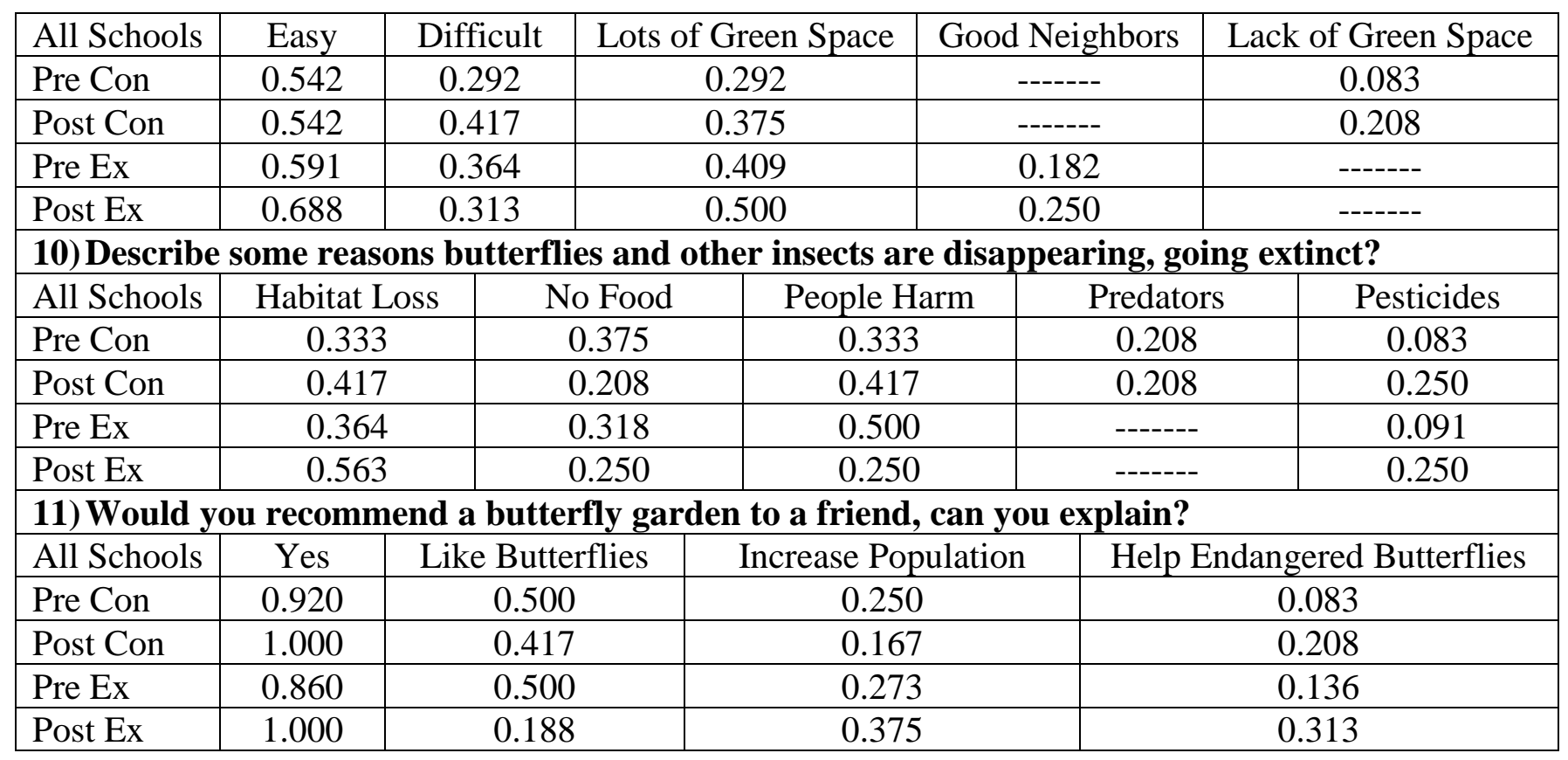


Table 4.8: The number of home gardens reported by students at Air Base K-8 Center (AB), North Hialeah Elementary (NHE), and Whispering Pines Elementary (WPE). The experimental groups increased the number of home gardens; however, both groups equally increased the number of butterfly gardens.

\begin{tabular}{|c|c|c|c|c|c|c|}
\hline Schools & $\mathbf{A B}$ & NHE & WPE & AB & NHE & WPE \\
\hline Treatment & \multicolumn{3}{|c|}{ Control Groups } & \multicolumn{3}{|c|}{ Experimental Groups } \\
\hline \# of Gardens (Presurvey) & 10 & 19 & 11 & 11 & 7 & 10 \\
\hline \# of Gardens (Postsurvey) & 10 & 17 & 11 & 13 & 10 & 13 \\
\hline Net Results (Gardens) & $\mathbf{0}$ & -2 & $\mathbf{0}$ & +2 & +3 & +3 \\
\hline \# of Butterfly Gardens (Presurvey) & 1 & 1 & 4 & 3 & 0 & 2 \\
\hline \# of Butterfly Gardens (Postsurvey) & 2 & 6 & 3 & 4 & 4 & 2 \\
\hline Net Results (Butterfly Gardens) & +1 & +5 & -1 & +1 & +4 & $\mathbf{0}$ \\
\hline
\end{tabular}




\section{FIGURES}

Figure 4.1: Map of school locations and their proximity to natural forest communities and environmentally endangered lands (EEL) sites. $\mathrm{AB}=$ Air Base K-8 Center; WPE $=$ Whispering Pines Elementary; NHE = North Hialeah Elementary .

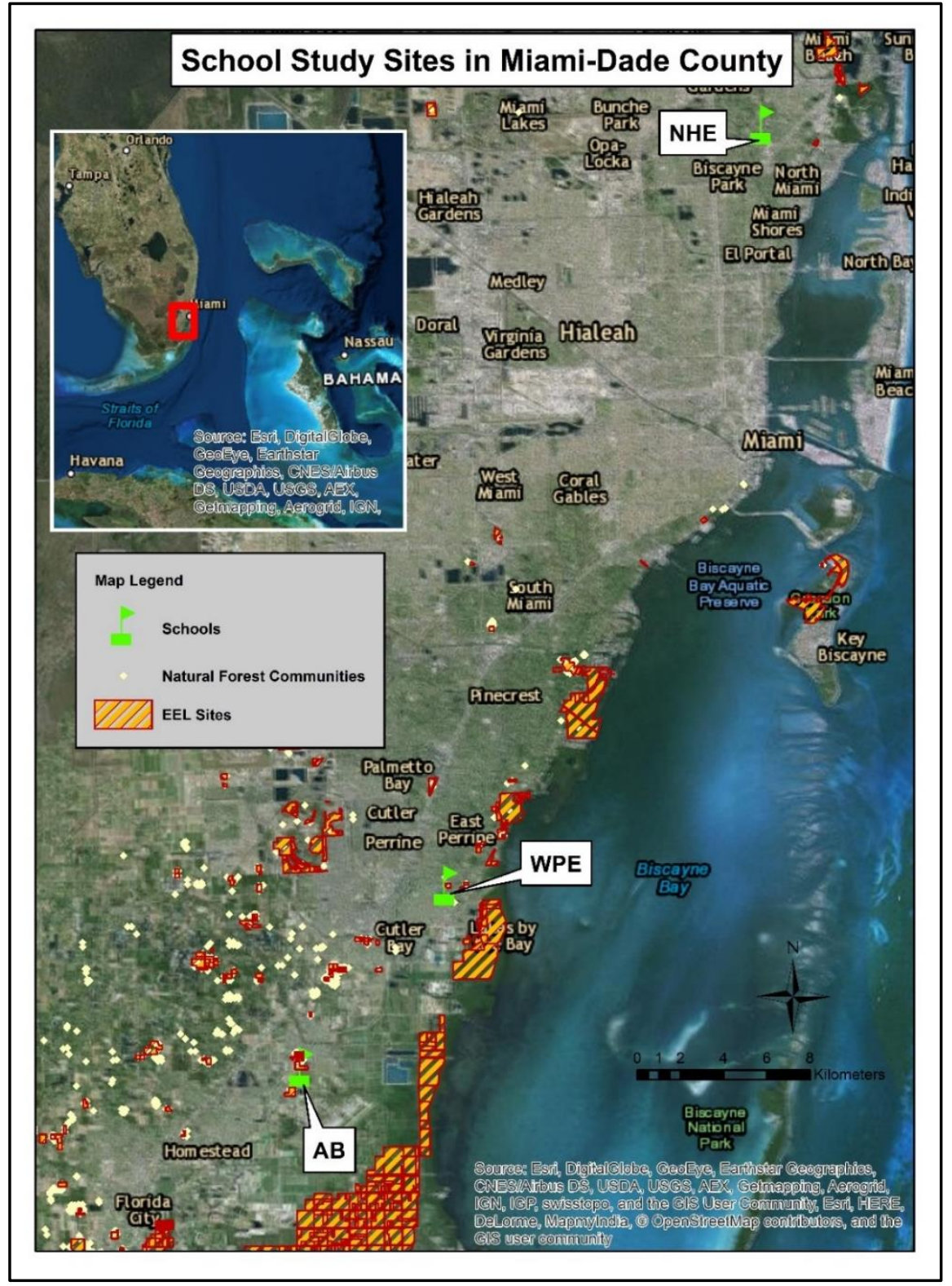


Figure 4.2: Teacher and $5^{\text {th }}$ grade students working together to construct the butterfly garden at North Hialeah Elementary School. See table 2 regarding the number of plants and plant species added to their garden.

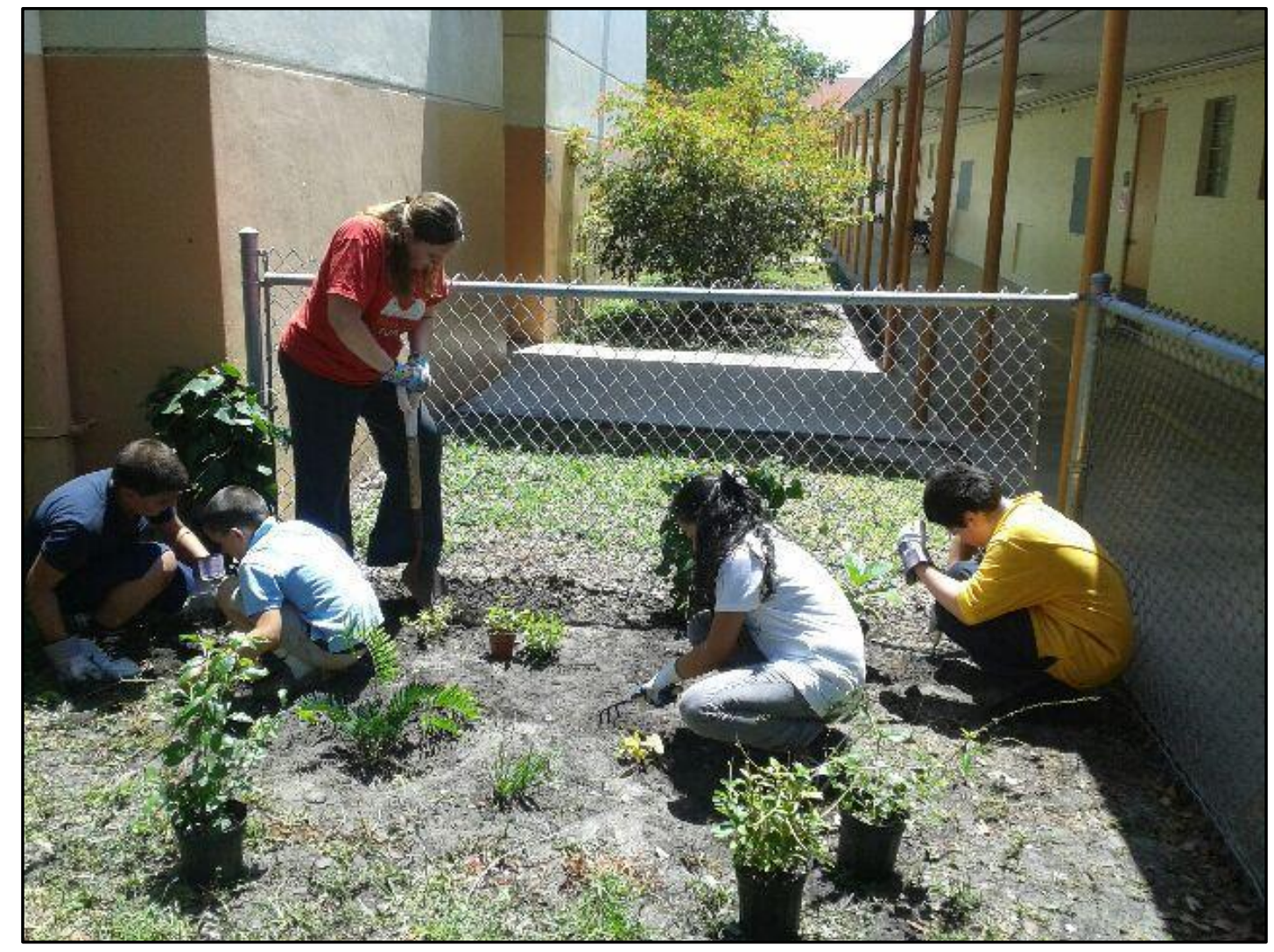


Figure 4.3: Two students at Whispering Pines Elementary School are reading the identification guide titled, "Butterflies of Southeast Florida: A Guide to Common and Notable Species” (Minno, 2014).

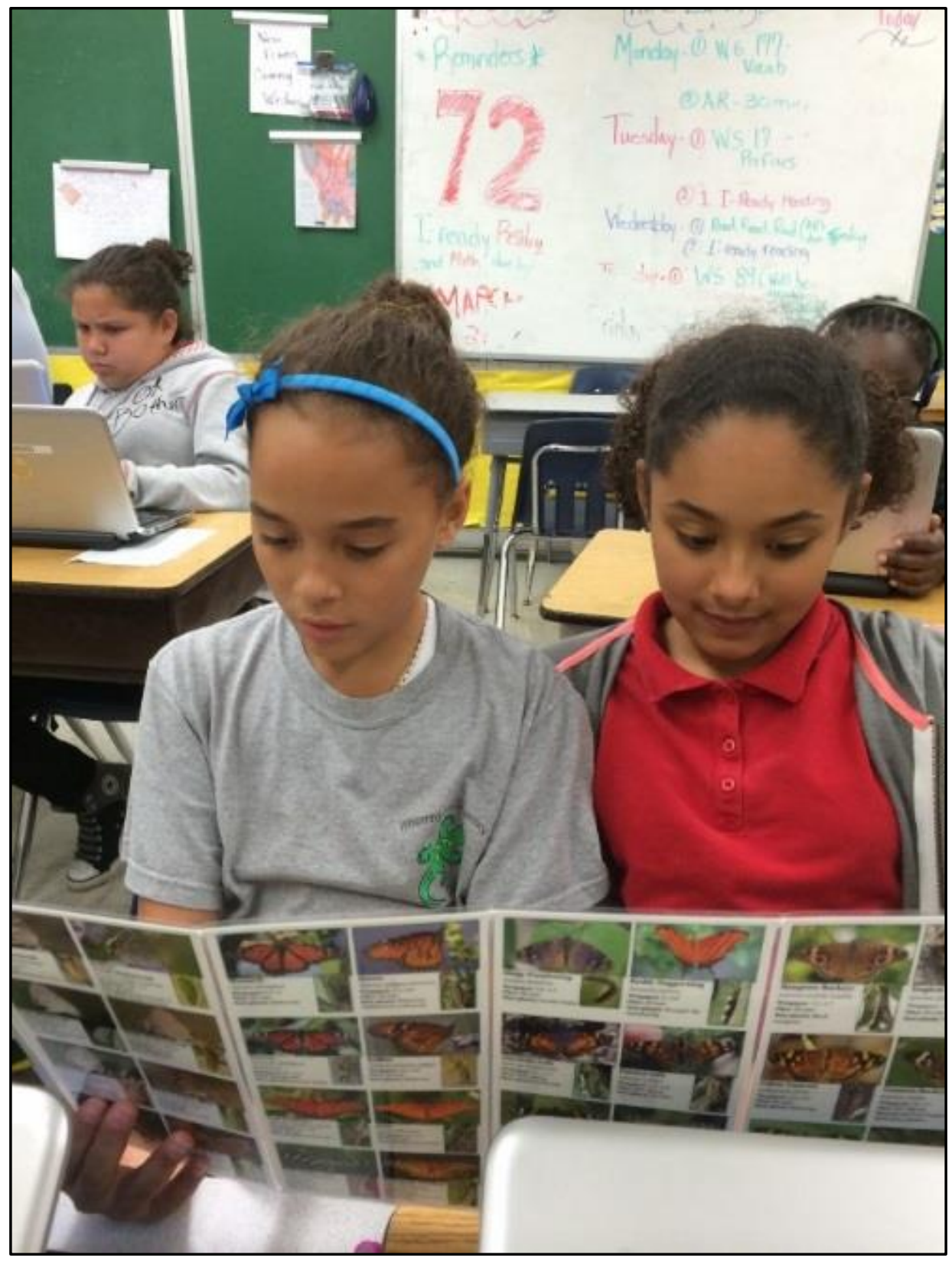


Figure 4.4: Combining all schools (mean $\pm \mathrm{SE}$ ), there was a significant increase in posttest scores for the experimental group; however, no significant increase for the control group (paired sample $t$-tests, experimental group: $d f=59, t=-6.301, \mathrm{p}<0.001$; control group: $d f=59, t=-1.239, \mathrm{p}=0.220)$. The intervention had a significant effect on post-test scores $(\mathrm{ANCOVA} ; d f=1 ; F=25.771, \mathrm{p}<0.001)$.

\section{All Schools}

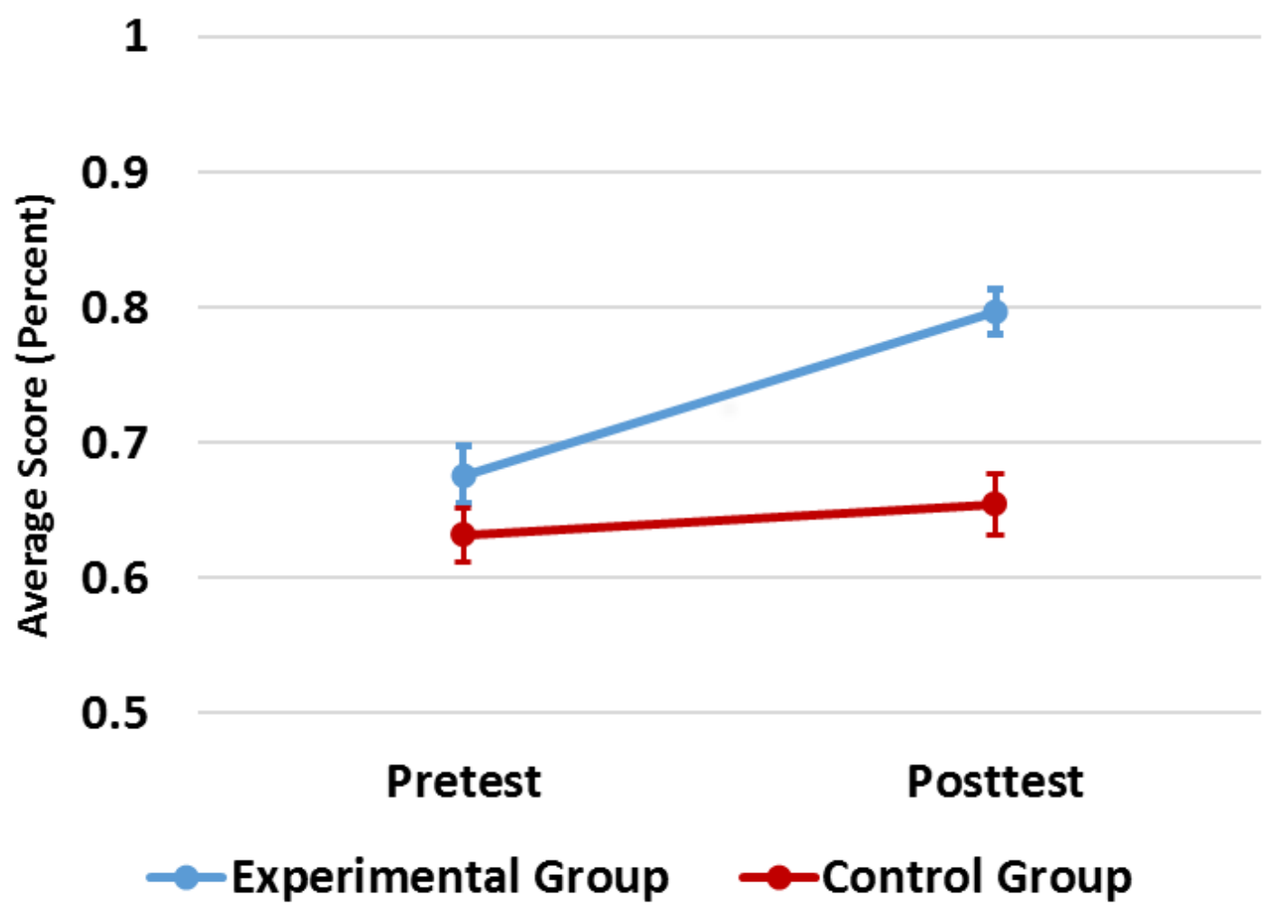


Figure 4.5: At Air Base K-8 Center (mean \pm SE), there was a significant increase on posttest scores for the experimental group; however, no significant increase for the control group (paired sample $t$-tests; experimental group: $d f=20, t=-3.871, \mathrm{p}<0.001$; control group: $d f=19, t=-0.937, p=0.360$ ). The intervention had a significant effect on posttest scores (ANCOVA; $d f=1 ; F=9.926, \mathrm{p}=0.003$ ).

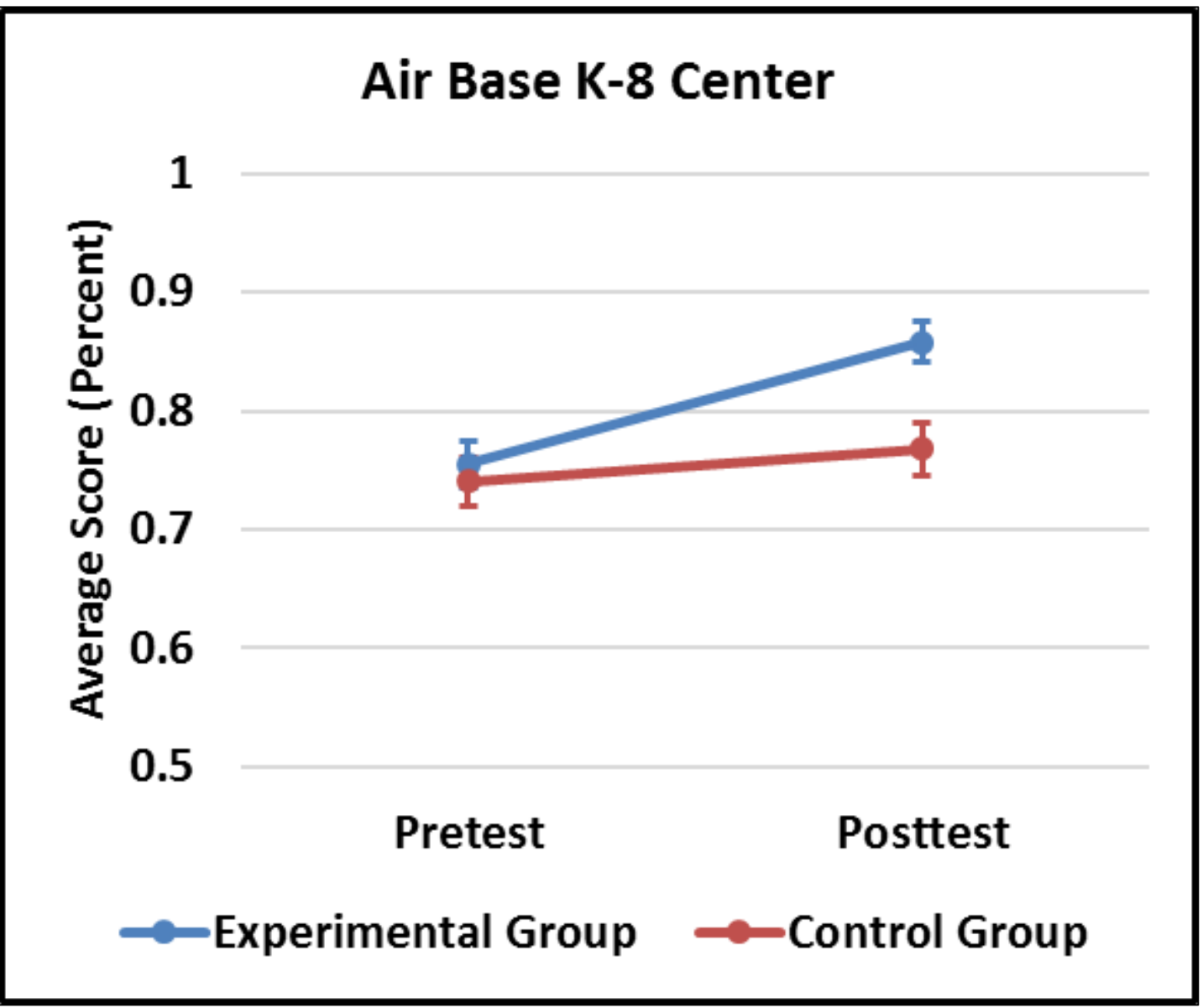


Figure 4.6: At North Hialeah Elementary School (mean \pm SE), there was a significant increase on posttest scores for the experimental group; however, no significant increase for the control group (paired sample $t$-tests; experimental group: $d f=17, t=-2.403, p=0.028$, control group: $d f=19, t=-0.573, p=0.573)$. The intervention had a significant effect on post-test scores (ANCOVA; $d f=1 ; F=6.203, \mathrm{p}=0.018$ ).

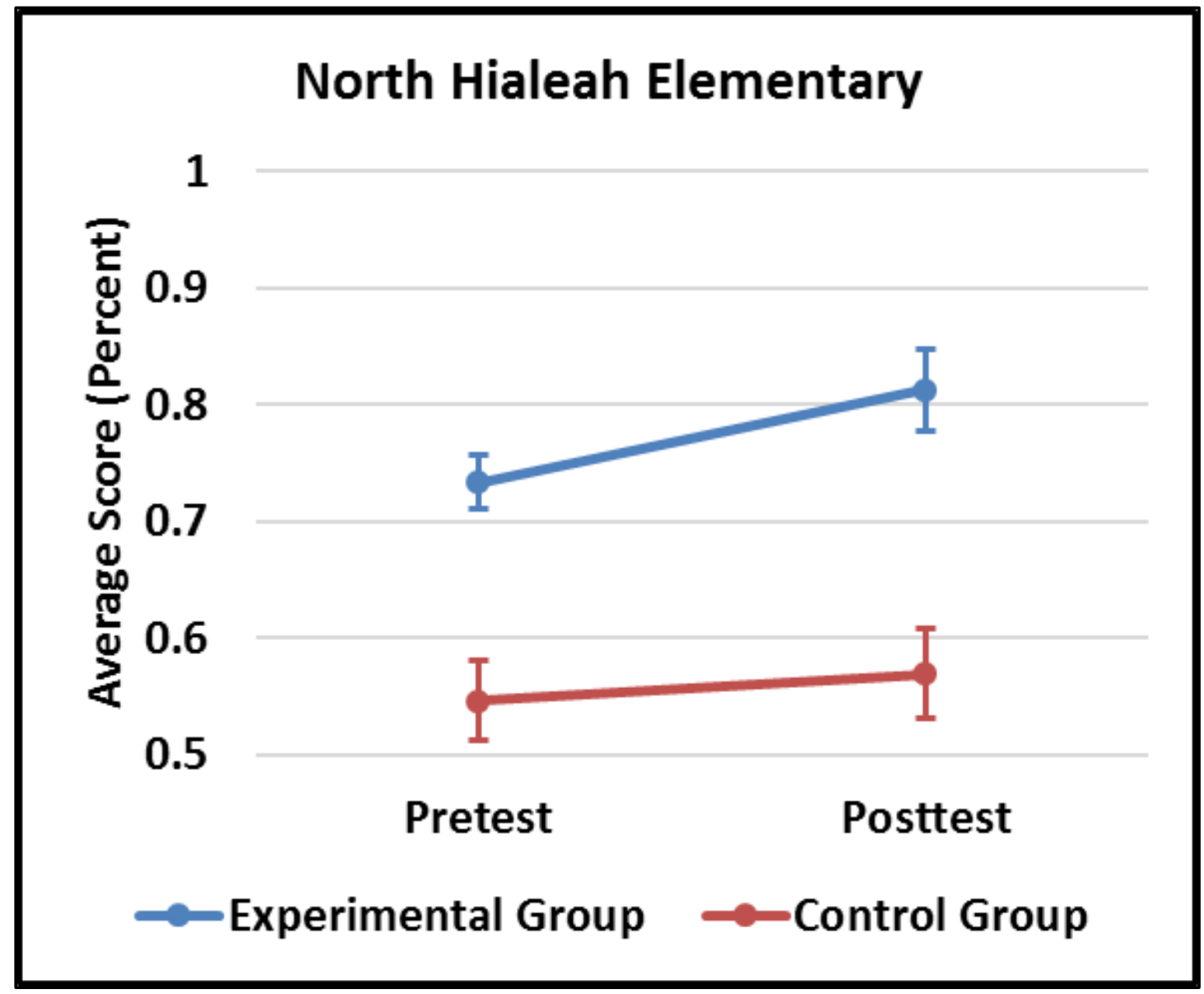


Figure 4.7: At Whispering Pines Elementary School (mean \pm SE), there was a significant increase on posttest scores for the experimental group; however, no significant increase for the control group (paired sample $t$-tests; experimental group: $d f=20, t=-4.705, p<$ 0.001, control group: $d f=19, t=-0.701, \mathrm{p}=0.492)$. The intervention had a significant effect on post-test scores (ANCOVA; $d f=1 ; F=10.986, \mathrm{p}=0.002)$.

\section{Whispering Pines Elementary}

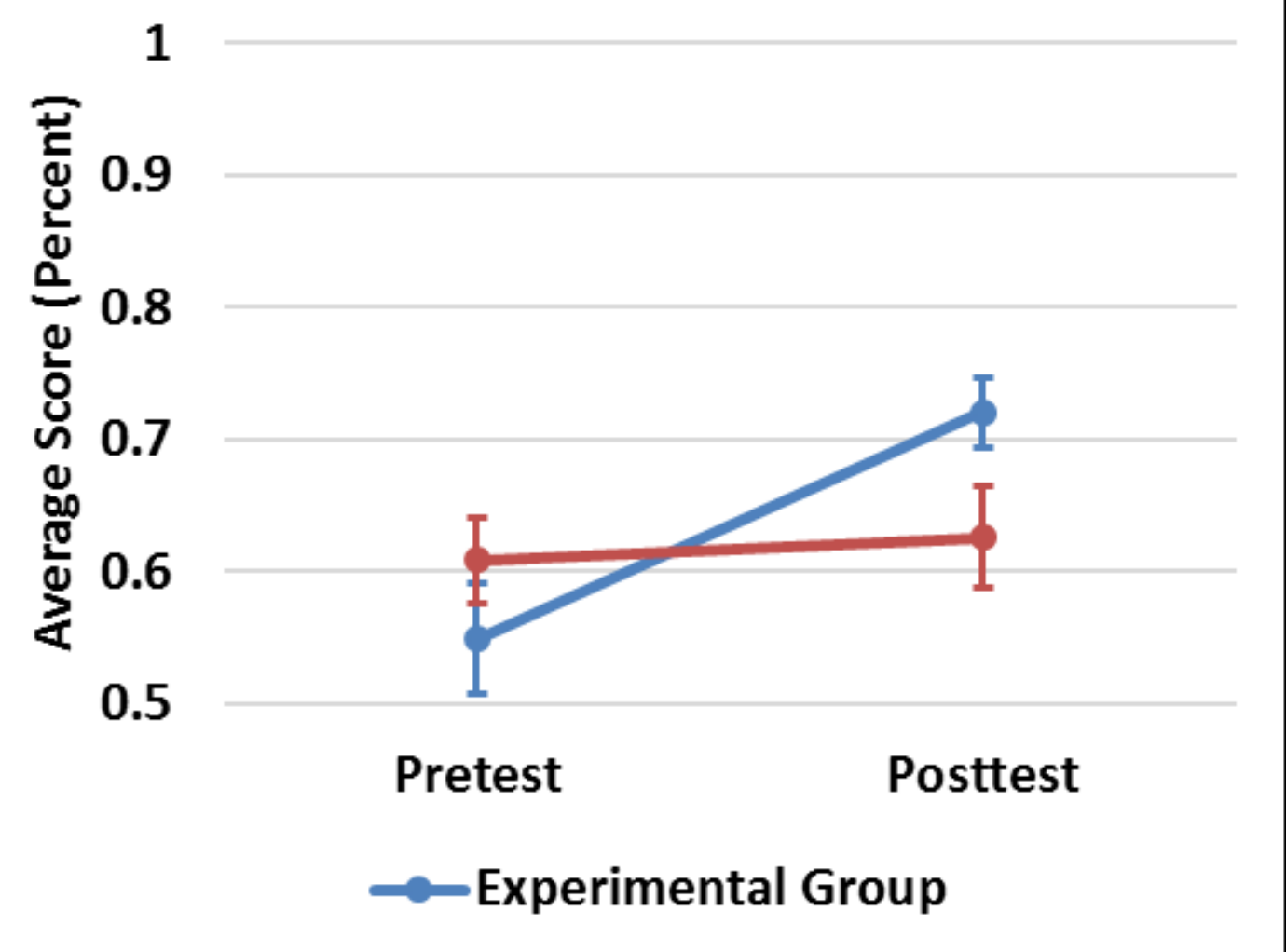


Figure 4.8: Boxplots depicting post-survey results for the five factors between the experimental and control groups (Scale: strongly agree $=5$, agree $=4$, neutral $=3$, disagree $=2$, strongly disagree $=1$ )

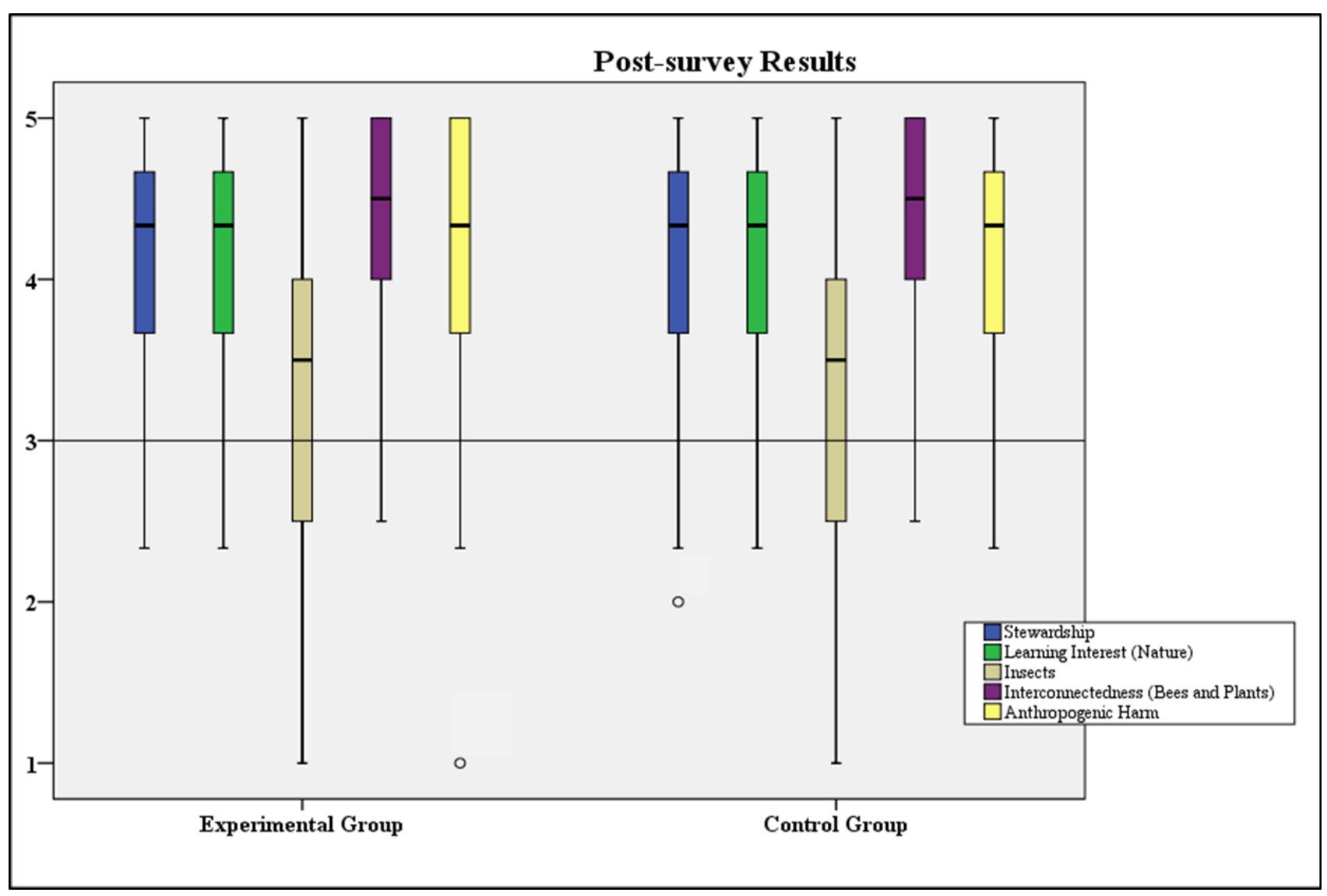




\begin{abstract}
APPENDICES
Appendix 4.1: Map of south Florida depicting the historic (outlined in green) and current range (outlined in red) of the federally endangered Schaus' swallowtail butterfly (Heraclides aristodemus ponceanus). The largest populations exist on islands in Biscayne National Park (outlined in blue). This map was enlarged (5' x $\left.3^{\prime}\right)$ for the activity titled, "Place the Schaus' swallowtail butterfly in the right habitat". This activity involved participation from the entire class. One blind-folded student was selected and placed at a random location in the classroom. The objective was to navigate the magnetic Schaus' swallowtail butterfly to suitable habitat (outlined in red) on the large map. Classmates were not allowed to verbally communicate to the blind-folded student; they were only able to navigate the blind-folded student using different toy instruments. Classmates were given 5-7 minutes to brainstorm and execute their strategic plan (Clayborn et al., 2017).
\end{abstract}




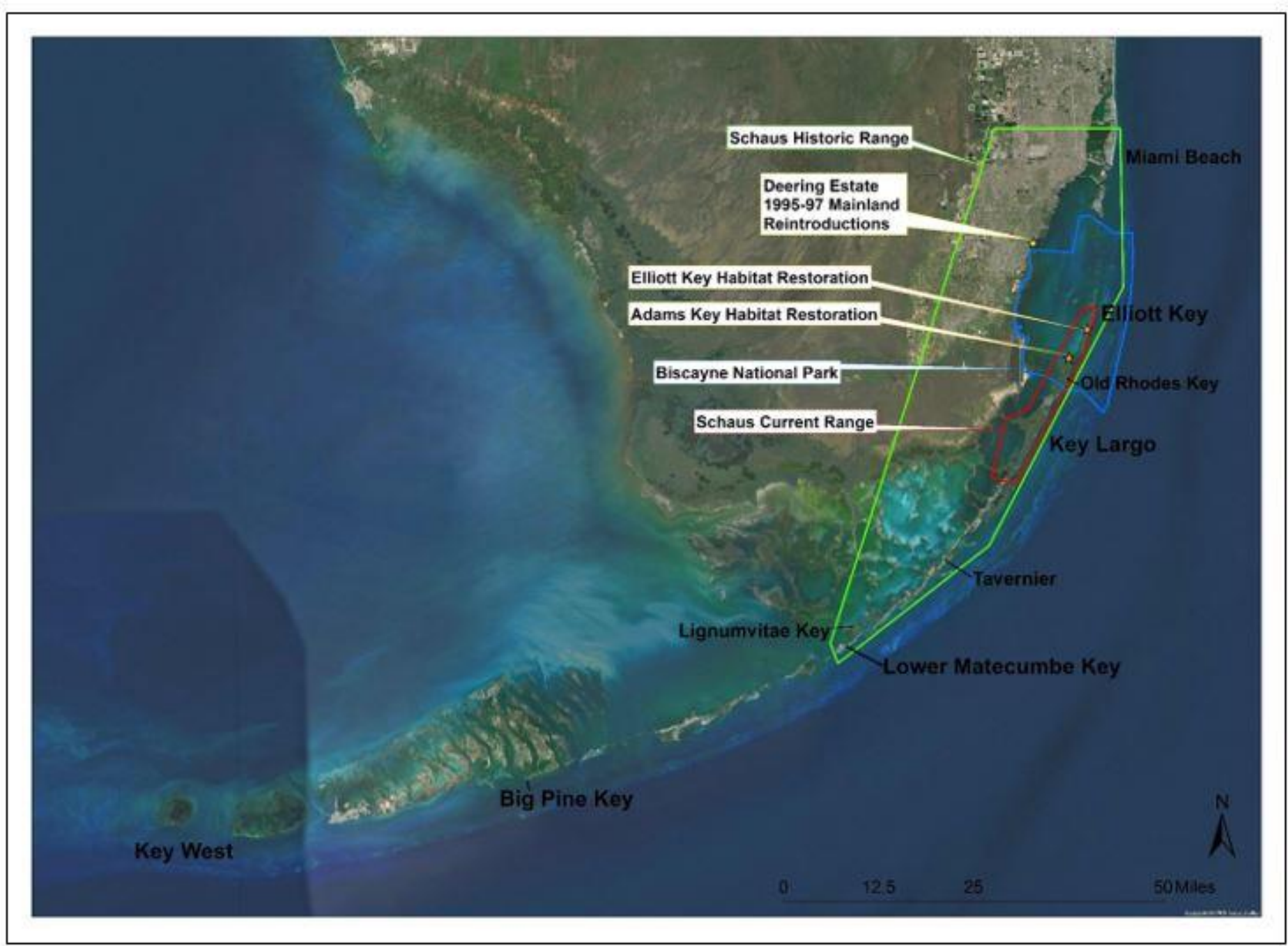


Appendix 4.2: Data observation sheet used by each student in the experimental treatment group. Data sheets were double-sided with detailed instructions on the back page. Parents and students were encouraged to report their findings using Twitter handles customized for each school. 


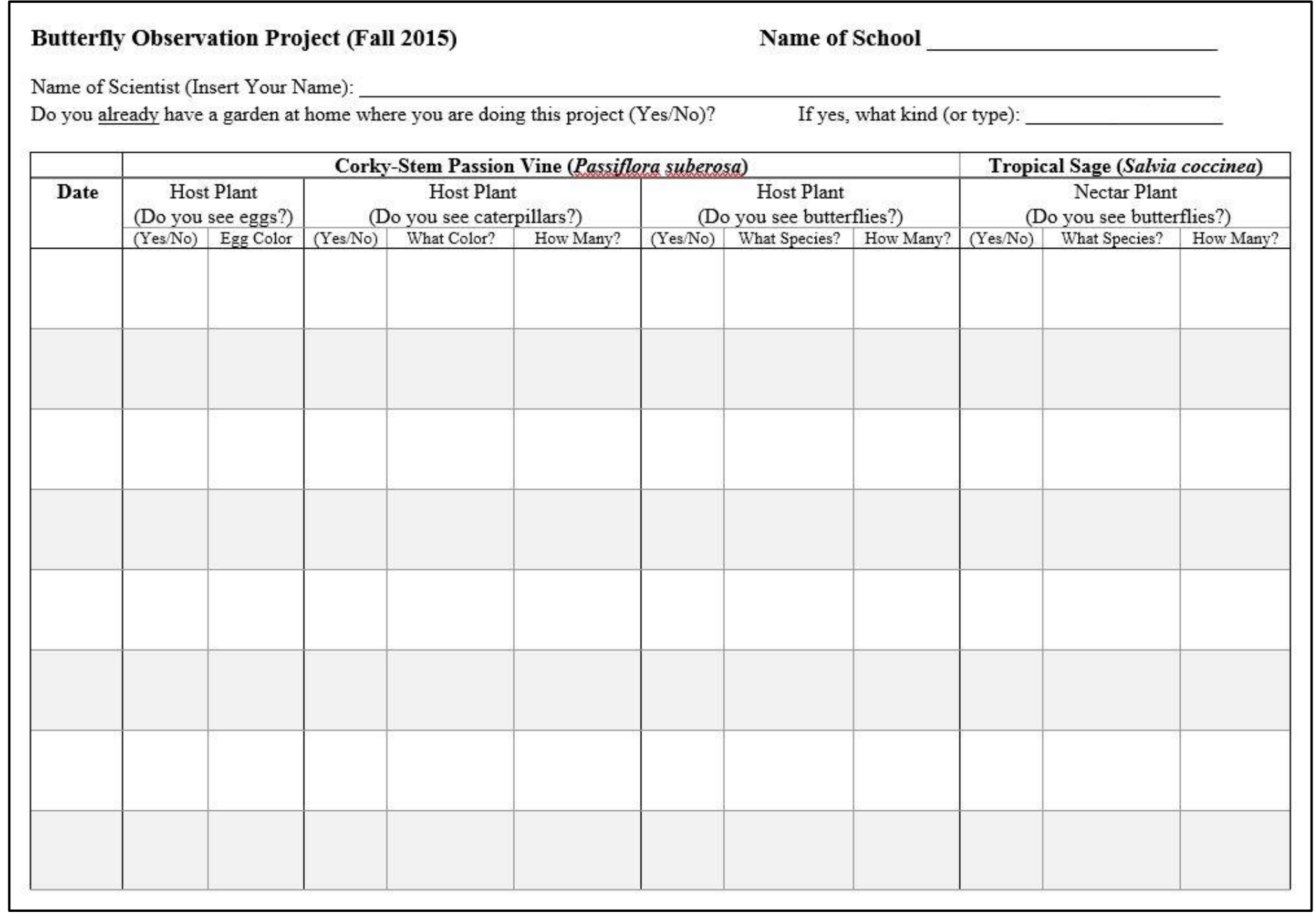




\begin{tabular}{|c|c|c|c|c|c|c|c|c|c|c|c|}
\hline \multirow{3}{*}{ Date } & \multicolumn{8}{|c|}{ Corky-Stem Passion Vine (Passiflora suberosa) } & \multirow{2}{*}{\multicolumn{3}{|c|}{$\begin{array}{c}\text { Tropical Sage (Salvia coccinea) } \\
\text { Nectar Plant } \\
\text { (Do you see butterflies on it?) }\end{array}$}} \\
\hline & \multicolumn{2}{|c|}{$\begin{array}{c}\text { Host Plant } \\
\text { (Do you see eggs?) }\end{array}$} & \multicolumn{3}{|c|}{$\begin{array}{c}\text { Host Plant } \\
\text { (Do you see caterpillars on it?) }\end{array}$} & \multicolumn{3}{|c|}{$\begin{array}{c}\text { Host Plant } \\
\text { (Do you see butterflies near it?) }\end{array}$} & & & \\
\hline & $(\mathrm{Yes} / \mathrm{No})$ & Egg Color & (Yes/No) & What Color? & How Many? & $(\mathrm{Yes} / \mathrm{No})$ & What Species? & How Many? & (Yes/No) & What Species? & How Many? \\
\hline & & & & & & & & & & & \\
\hline & & & & & & & & & & & \\
\hline & & & & & & & & & & & \\
\hline & & & & & & & & & & & \\
\hline
\end{tabular}

\section{Review}

You are nurturing two native plant species that attract butterflies (Corky-Stem Passion Vine and Tropical Sage). They are drought-tolerant when established in the ground. Both plant species can live in shady environments; however, they grow faster and flower sooner with more sun. If you attract caterpillars (Yay!!!) do not worry about the spikes on their skin (spikes are soft). Spikes are present to scare predators and do not cause an irritation (it is a bluff). The caterpillars are not dangerous to you. Remember to have fun and use your identification guides to ID butterflies and caterpillars. The project will end in December ( $2^{\text {nd }}$ week). If you have any questions, comments, or concerns do not hesitate to contact me via email (iclav010@fiu.edu). Thank you for your time and participation in this project. If possible, share photos of your plants at \#airbasek8butterflies.

\section{Instructions}

1) Make sure you water the plants and keep the soil moist in the pot.

2) Check the plants for butterfly activity on a weekly basis (Saturday or Sunday during daylight hours)

3) Write down your observations on this data sheet using the southeast Florida butterfly identification guide.

4) Be patient, butterflies might take longer than expected time to find your plants.

\section{Websites with information about butterflies and butterflies in south Florida}

1) miamiblue .org (North American Butterfly Association Miami Blue Chapter)

2) https://edis.ifas.ufl.edu/topic_butterfly_gardening (University of Florida IFAS Extension Butterfly Gardening) 


\section{CONCLUSIONS AND FUTURE DIRECTIONS}

Biscayne National Park (BNP) and North Key Largo are the last remaining sanctuaries for the federally endangered Schaus' swallowtail butterfly (Daniels, 2014; Minno, 2015). Biscayne National Park supports the largest population of Schaus' swallowtail butterflies in south Florida. The butterflies' fate in the near future remains optimistically positive because of substantial work being conducted by universities, federal and state government agencies, local non-governmental organizations, and the many passionate volunteers concerned with the butterflies' preservation. Scientists and staff at the University of Florida have released captive-bred butterflies to supplement wild populations (Daniels, 2014; Minno, 2015). Scientists and staff at the National Park Service South Florida Caribbean Network and BNP collaborated to restore and enhance dry forest habitat in BNP by removing invasive plant species and increasing the number of host plants for the Schaus' swallowtail butterfly (Whelan et al., 2013; Whelan and Atkinson, 2015). Because butterfly populations can fluctuate year to year (Thomas et al., 1994; Daniels, 2014; Minno, 2015), the efficacy of these projects are being monitored on a yearly basis (Daniels, 2014; Minno, 2015; Whelan and Atkinson, 2015). Conclusive statements should be evaluated with caution as more data are collected each year. Explicit long-term data detailing population dynamics and potential carrying capacity in the Florida Keys will provide evidence-based information to determine the efficiency of extinction prevention programs for the Schaus' swallowtail butterfly. This preservation model can be applied to other imperiled butterflies in south Florida.

Wild lime was more robust in the restoration sites than sea torchwood (Table 2.2). Sea torchwood survivorship was over 50\% until 2017 when the majority of sea 
torchwood along Adams Key Breezeway was recorded as dead and lowered the overall survivorship to $47.5 \%$ (Table 2.2). Overall, the effects of canopy cover, elevation, and relative soil depth were negligible in sea torchwood and wild lime survivorship (Table 2.8). Elevation had a substantial effect on both sea torchwood and wild lime growth (Table 2.7). The imminent threat of sea level rise (SLR) is an ominous threat to the remaining suitable habitat for the Schaus' swallowtail butterfly (Bibb and Hughes, 2007). Spatial analyses revealed dry forest ecosystems in North Key Largo are better situated against gradual rise in sea level because of higher elevation (Figures 2.15-2.18). Dry forest ecosystems in BNP occupy areas that are lower in elevation; consequently, they most likely will succumb to rising seas at a faster rate (Table 2.10).

Ants are important predators in tropical forest ecosystems (Floren et al., 2002). Swallowtail butterflies express various phenotypic defense mechanisms; however, invertebrate predators are more likely deterred by defensive tactics such as chemicals stored in their osmeteria, physical head butts to attacker(s), and leaf abortion to escape predators (Honda, 1983; Nafus, 1993; Frankfater et al., 2009). These defensive strategies may be less effective against aggressive, exotic ants (Forys et al., 2001). Exotic, predatory ants can overwhelm and eat their prey to extinction when prey populations reach dangerously low numbers (Schoener et al., 2001; Porter and Savignano, 1990). Ants, particularly the exotic $P$. gracilis, may pose a significant threat to Schaus' swallowtail caterpillars in the Florida Keys (Bibb and Hughes, 2007; Wetterer, 2010). Pseudomyrmex gracilis was the most common ant on sea torchwood and wild lime in BNP (Table 3.2; Figure 3.3) and aggressively attacked and removed early instar caterpillars (Table 3.5; Clayborn and Koptur, 2017). Unfortunately, we know little about 
the rate of encounters between $P$. gracilis and Schaus' swallowtail caterpillars. Continued investigations should take steps to quantify encounter rates between $P$. gracilis and other predatory threats against Schaus' swallowtail caterpillars. Long-term quantitative research is necessary to assess potential risks imposed by exotic ants and other predators. High-definition video surveillance can reveal predation events in natural settings day and night over an extended period of time to more accurately assess the effects of predator (particularly ants) interactions with all immature stages of Schaus' swallowtail caterpillars in the Florida Keys (Grieshop et al., 2012). Different predators may be more deleterious at different life-history stages of butterflies (Frankfater et al., 2009; Seifert et al., 2015). This method of investigation (HD video surveillance) can revolutionize scientific techniques that assemble life-histories of rare invertebrate species under natural conditions with real-time information.

Community engagement and advocacy for insect preservation and habitat conservation are integrated in exposure, education, and experience (Miller, 2005; Sobel, 2005; Guiney and Oberhauser, 2009). The controlled-investigation at three schools in Miami-Dade County, revealed native plant butterfly gardens and integrated insect curriculum units increased students' content knowledge about south Florida ecosystems and butterflies (Figure 4.4). Attitudinal shifts were minimal because students were already interested in the environment, plants, and animals; however, they were less interested in insects (Table 4.6; Figure 4.8). Interview data revealed students were less apprehensive against butterflies and understood the effects of urbanization against wildlife during post-interviews (Table 4.7). Eventually, this led to the construction of more butterfly gardens at home (Table 4.8). In the future, we would like to use the 
Schaus' swallowtail butterfly and Heraclides aristodemus congeners as flagship species in Cuba, Miami-Dade County, and Puerto Rico and expose teachers, students, and parent chaperones to habitat restoration projects in tropical dry forest ecosystems. The integrated insect curriculum unit would be implemented at each school. A bilingual communication network between schools will allow teachers and students to communicate with other students, locally and internationally. Students and parents can apply these servicelearning projects in the classroom, at home, and in the field, thus leading to higher academic achievement and social awareness about environmental quality.

Collaboration with scientists and communities in Cuba and Puerto Rico presents an opportunity to share ideas and innovate methods to increase our collective knowledge about the Schaus' swallowtail butterfly and close $H$. aristodemus congeners in dry forest ecosystems. A better understanding of rare butterfly dynamics in the Florida Keys and Greater Antilles presents an opportunity to preserve their existence and thwart extinction. Through education, butterflies can be used as flagship species and initiate a generational paradigm shift towards coexistence between humans and dry forest ecosystems.

\section{LITERATURE CITED}

Bibb, K., and Hughes, P. 2007. United States Fish and Wildlife Service Schaus Swallowtail butterfly (Heraclides aristodemus ponceanus) 5-Year review: Summary and evaluation. United States Fish and Wildlife Service, Southeast Region, South Florida Ecological Services Office, Vero Beach, Florida. Available online at http://ecos.fws.gov/docs/five_year_review/doc1983.pdf. Accessed 20 February 2017.

Clayborn, J., and Koptur, S. 2017. Mortal combat between ants and caterpillars: an ominous threat to the endangered Schaus swallowtail butterfly (Heraclides aristodemus ponceanus) in the Florida Keys, USA. Journal of Insect Conservation, 21(4): 689-702. 
Daniels, J.C. 2014. Conservation matters: Status and conservation of the federally endangered Schaus swallowtail butterfly. News of the Lepidopterists' Society 56: 138-139.

Floren, A., Biun, A., and Linsenmair, K.E. 2002. Arboreal ants as key predators in tropical lowland rainforest trees. Oecologia, 131(1): 137-144.

Forys, E.A., Quistorff, A., and Allen, C.R. 2001. Potential fire ant (Hymenoptera: Formicidae) impact on the endangered Schaus swallowtail (Lepidoptera: Papilionidae). Florida Entomologist, 84(2): 254-258.

Frankfater, C., Tellez, M.R., and Slattery, M. 2009. The scent of alarm: Ontogenetic and genetic variation in the osmeterial gland chemistry of Papilio glaucus (Papilionidae) caterpillars. Chemoecology, 19(2): 81-96.

Grieshop, M.J., Werling, B., Buehrer, K., Perrone, J., Isaacs, R., and Landis, D. 2012. Big brother is watching: Studying insect predation in the age of digital surveillance. American Entomologist, 58(3): 172-182.

Guiney, M.S., and Oberhauser, K.S. 2009. Insects as flagship conservation species. Terrestrial Arthropod Reviews, 1(2): 111-123.

Honda, K. 1983. Defensive potential of components of the larval osmeterial secretion of papilionid butterflies against ants. Physiological Entomology, 8(2): 173-179.

Miller, J.R. 2005. Biodiversity conservation and the extinction of experience. Trends in Ecology and Evolution 20(8): 430-434.

Minno, M.C. 2015. 2015 Schaus' swallowtail (Heraclides aristodemus ponceanus) monitoring on Key Largo, Florida. Southern Lepidopterists' News, 37(3): 106-107.

Nafus, D.M. 1993. Movement of introduced biological-control agents onto nontarget butterflies, Hypolimnas spp. (Lepidoptera, Nymphalidae). Environmental Entomology, 22(2): 265-272.

Porter, S.D., and Savignano, D.A. 1990. Invasion of polygyne fire ants decimates native ants and disrupts arthropod community. Ecology, 71: 2095-2106.

Schoener, T.W., Spiller, D.A., and Losos, J.B. 2001. Predators increase the risk of catastrophic extinction of prey populations. Nature, 412(6843): 183-186.

Seifert, C.L., Lehner, L., Adams, M., and Fiedler, K. 2015. Predation on artificial caterpillars is higher in countryside than near-natural forest habitat in lowland southwestern Costa Rica. Journal of Tropical Ecology, 31(3): 281-284. 
Sobel, D. 2005. Place-Based Education: Connecting Classrooms and Communities. The Orion Society, Great Barrington, MA.

Thomas, J.A., Moss, D., and Pollard, E. 1994. Increased fluctuations of butterfly populations towards the northern edges of species' ranges. Ecography, 17(3): 215220.

Wetterer, J.K. 2010. Worldwide spread of the graceful twig ant, Pseudomyrmex gracilis (Hymenoptera: Formicidae). Florida Entomologist, 93(4): 535-540.

Whelan, K., Clayborn, J., Giannini, H.C., Pernas, T., and Perry, C. 2013. Planting to save the Schaus Swallowtail Butterfly: The National Park Service's Schaus Swallowtail Habitat Enhancement Project. Fairchild Tropical Botanic Garden, 68(1): 42-43.

Whelan, K.R.T., and Atkinson, A. 2015. Schaus Swallowtail Butterfly (Heraclides aristodemus ponceanus) Habitat Enhancement in Biscayne National Park. South Florida Coastal Program Project Progress Report. NPS/SFCN. National Park Service, Palmetto Bay, Florida. 
VITA

\title{
JAESON TYRONE CLAYBORN
}

\author{
Born, Cleveland, Ohio
}

2002-2006

B.S., Biology, Minor in Environmental Studies

Eckerd College

St. Petersburg, Florida

2008-2011

M.S., Environmental Science and Policy

University of South Florida St. Petersburg

St. Petersburg, Florida

2011-2018

Doctoral candidate in Biological Sciences

Florida International University

Miami, Florida

Teaching Assistant, 2011-2017

Florida International University

Miami, Florida

Dissertation Year Fellowship, 2017

University Graduate School, Florida International University

Miami, Florida

\section{PUBLICATIONS AND PRESENTATIONS}

Clayborn, J., Koptur, S., O’Brien, G., and Whelan, K. 2017. The Schaus Swallowtail Habitat Enhancement Project: An Applied Service-Learning Project Continuum from Biscayne National Park to Miami-Dade County Public Schools. Southeastern Naturalist, 16(10): 26-46.

Clayborn, J., and Koptur, S. 2017. Mortal combat between ants and caterpillars: An ominous threat to the endangered Heraclides aristodemus ponceanus in the Florida Keys, USA. Journal of Insect Conservation, 21(4): 689-702.

Clayborn, J., O’Brien, G., and Medina, M. 2017. School gardening with a twist using fish: Encouraging educators to adopt aquaponics in the classroom. Applied Environmental Education \& Communication, 16(2): 93-104.

O’Brien, G., Sparrow, K., Morales, J., Clayborn, J. 2015. Re-orienting a Science Methods Course to Prepare Sustainability Literate K-6 Pre-service Teachers: A Mixed Methods Investigation. In: Stratton, S., Hagevik, R., Feldman, A., Bloom, M. (eds) Educating 
Science Teachers for Sustainability. ASTE Series in Science Education. Springer, Cham, pp. 205-234.

Cassill, D.L., Casella, A., Clayborn, J., Perry, M., and Lagarde M. 2015. What can ants tell us about collective behavior during a natural catastrophe? Journal of Bioeconomics, 17(3): 255-270.

Clayborn, J. Butterfly gardening at Miami urban schools: Plugging students into nature through habitat rehabilitation on school grounds. Invited speaker, The Dade Chapter Florida Native Plant Society, Pinecrest, Florida, 10/2017.

Clayborn, J. (1) El aumento del nivel del mar vs. el mejoramiento del habitat de la planta huesped, Heraclides aristodemus ponceanus; Paradoja de cambio climatico? (2) El mortal kombat entre hormigas exóticas y especies de Heraclides en el bosque seco tropical en los cayos de Florida. IX Congreso de la Biodiversidad Caribeña, Santo Domingo, Dominican Republic, 01/2017.

Clayborn, J. School gardening with a twist using fish: Encouraging educators to adopt aquaponics in the classroom. Botanical Society of America, Savannah, Georgia, 07/2016.

Clayborn, J. The Schaus Swallowtail Habitat Enhancement Project: An Applied ServiceLearning Project Continuum from Biscayne National Park to Miami-Dade County Public Schools. Annual Meeting of the Florida Academy of Sciences, St. Petersburg, Florida, 03/2016. Outstanding Graduate Student Oral Presentation/Science Teaching Section.

Clayborn, J. Monarch conservation in a changing world: Milkweed, Michoacán, and Miami. Invited speaker, Plant Talk (Florida International University), Miami, Florida, $02 / 2016$.

Clayborn, J. El mortal kombat entre hormigas y especies de Heraclides en los Cayos de Florida. V Simposio, Ecología, Sociedad y Medio Ambiente “Ecovida 2015”, Pinar del Río, Cuba, 12/2015.

Clayborn, J. Aumento del nivel del mar versus mejoramiento de habitat de Heraclides aristodemus ponceanus, una paradoja? Poster Presentation, Cuba Primera Circular Evento Cientifico Internacional "Utowana 2014”, Cienfuegos, Cuba, 11/2014.

Clayborn, J. Butterflies and Habitat Restoration: What We Can Do. Roundtable Discussion, 43rd Annual North American Association for Environmental Education (NAAEE), Ottawa, Canada, 10/2014.

Clayborn, J. The Schaus and Coastal Hardwood Hammocks. Florida Marine Science Educators Association (FMSEA), St. Petersburg, Florida, 05/2012. 